\title{
TOTAL SYNTHESIS OF VARIOLIN B
}

\author{
A Thesis \\ Submitted in Partial Fulfilment \\ of the Requirements for the Degree \\ of \\ Doctor of Philosophy in Chemistry \\ at the \\ University of Canterbury
}

by

Regan J. Anderson

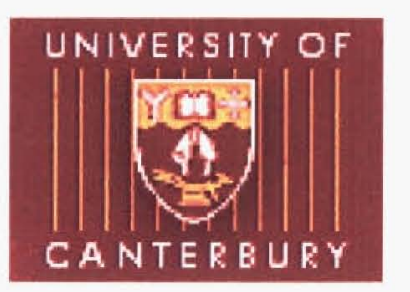

February 2002 


\section{Acknowledgements}

I would like to thank Dr Jonathan Morris for his supervision and guidance throughout this project. His approachable manner and hands-on supervision have been greatly appreciated. I am also grateful for the hours he has put into checking and revising this thesis.

Thanks also go to the past and present members of the Morris group, for making my time here enjoyable and memorable. Many laughs have been had-usually at the expense of one another. Particular mention should be made of Bungard, Darbs and Muscroft, alongside whom most of this work has been carried out.

The technical staff in the Chemistry Department have been most friendly and helpful. In particular, I would like to thank Bruce Clark for carrying out all mass spectrometry analyses and assistance with their interpretation. Thanks also to Gill Ellis for biological testing of compounds and helpful discussions in this area.

The University of Canterbury, the New Zealand Vice-Chancellors' Committee and Pharma Mar, SA are gratefully acknowledged for financial assistance.

Thanks to Mum and Dad for their encouragement throughout my $\mathrm{PhD}$. Especially in the last few months, their support has been valued and my flatmates and I have enjoyed their cooking.

Mostly, thank you Becs for your love and support over the last 18 months. Your continual interest in what I have been doing has always been appreciated. Thanks for putting up with not seeing much of me and for bringing me food and goodies when I needed them. 


\begin{abstract}
This thesis describes the development of methodology which has led to the total synthesis of variolin $\mathrm{B}$, a marine alkaloid with potent antitumour properties. In Chapter One, a brief summary of the association of organic synthesis and marine natural products is provided. This is followed by an account of the variolin family of natural products and synthetic efforts that have been directed towards them. A new strategy is proposed that exploits the hidden symmetry in variolin $\mathrm{B}$.
\end{abstract}

Chapter Two covers investigations into the synthesis of the variolin skeleton using a variety of reagent systems. A rapid entry into the core structure of the variolins was found, with the key step being a deoxygenation/cyclisation protocol, which was mediated by the combination of triethylsilane and trifluoroacetic acid. Evidence was obtained to suggest an unexpected mechanism for this reaction. Straightforward functional group manipulation led to a synthesis of deoxyvariolin B.

In Chapter Three, the previously established methodology was applied to an alternative starting material, appropriate for a synthesis of variolin B. However, the process was low-yielding and gave variable results. Some modifications to the methodology led to a reliable and efficient procedure for the synthesis of the variolin skeleton, which was subsequently transformed to variolin $\mathrm{B}$. The synthesis proceeded in a total of eight linear steps, with an overall yield of $13 \%$.

The methodology which has been developed lends itself to the production of analogues of the variolins. The synthesis and biological testing of some analogues are presented in Chapter Four, along with a discussion on the structural features which confer biological activity on variolin $\mathrm{B}$.

A brief summary of the work described in this thesis and potential future studies is given in Chapter Five. 


\section{Table of Contents}

\section{Chapter One Introduction}

1.1 Marine Natural Products 2

1.1.1 The State-of-Play in Marine Natural Products 9

1.2 The Variolins: a Unique Class of Marine Alkaloids 13

1.2.1 Introduction 13

1.2.2 Structure Elucidation 13

$\begin{array}{lll}1.2 .3 & \text { Biological Activity } & 15\end{array}$

1.2.4 Related Synthetic Work 16

1.2.5 Synthetic Efforts Towards the Variolins 20

$\begin{array}{ll}\text { 1.2.6 Work Described in this Thesis } & 27\end{array}$

\section{Chapter Two Symthesis of the Variolin Core and Deoxyvariolin $B$}

2.1 Introduction 31

2.2 Synthesis of Triarylmethanol 2.3 32

2.3 Formation of the Variolin Core $\quad 44$

2.3.1 Radical Deoxygenation of Triarylmethanol 2.3 44

2.3.2 Ionic Hydrogenolysis of Triarylmethanol $2.3 \quad 48$

$\begin{array}{lll}2.3 .3 & \text { Summary } & 70\end{array}$

2.4 Elaboration of the Variolin Core 2.1 to Deoxyvariolin B 71

2.4.1 Direct Substitution of Thiomethyl Groups $\quad 71$

2.4.2 Oxidation/Nucleophilic Substitution of Thiomethyl Groups 75

2.4.3 Removal of the $p$-Methoxybenzyl Protecting Groups 81

2.5 Summary $\quad 86$

Chapter Three The Total Synthesis of Variolin B

3.1 Synthesis of Triarylmethanol 1.65

3.1.1 Synthesis of the Acid Chloride 1.67 90

3.1.2 Addition of the Pyrimidine to Acid Chloride 1.67 91 
3.2.1 Strategy

3.2.2 Synthesis of the Pyridine 101

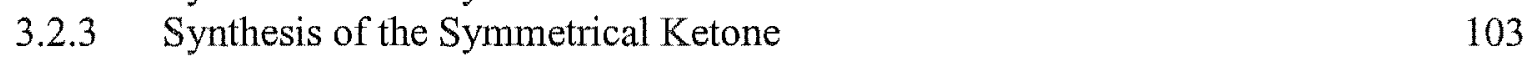

3.2.4 Formation of the Triarylmethanol 1.65 104

$\begin{array}{lll}3.2 .5 & \text { Summary } & 108\end{array}$

$\begin{array}{lll}3.3 & \text { Formation of the Variolin Core } 1.63 & 109\end{array}$

3.3.1 TFA/TES "Tonic Hydrogenolysis" of Triarylmethanol 1.65 109

3.3.2 An Alternative Substrate for the TFA/TES Reaction 113

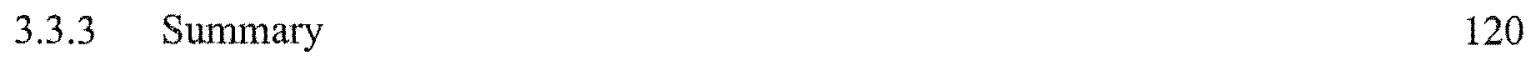

$\begin{array}{ll}3.4 & \text { Synthesis of Variolin B }\end{array}$

3.4.1 Preliminary Studies in Deprotection of the Methoxy Group 121

3.4.2 Oxidation/Nucleophilic Substitution of Thiomethyl Groups 124

3.4.3 Deprotection of the Methoxy Group 127

3.4.4 Removal of the $p$-Methoxybenzyl Protecting Groups $\quad 128$

$\begin{array}{llr}3.5 & \text { Summary } & 133\end{array}$

Chapter Four Structure-Activity Relationships and Synthesis of Analogues

$\begin{array}{lll}4.1 & \text { Introduction } & 136\end{array}$

$\begin{array}{lll}\text { 4.1.1 Definitions } & 136\end{array}$

4.1.2 Biological Data of Synthetic Variolin B 137

4.2 Structure-Activity Relationships in the Variolins 138

$\begin{array}{lll}\text { 4.2.1 Introduction } & 138\end{array}$

4.2.2 Variation in Group Z 139

4.2.3 Variation in Groups X and Y 144

4.3 Semi-Rational Production of Variolin Analogues $\quad 149$

$\begin{array}{llr}4.3 .1 \quad \text { Background } & 149\end{array}$

4.3.2 Synthesis $\quad 150$

4.3.3 P388 Assay results 154

4.4 Summary 156 
Chapter Five General Summary and Future Studies

5.1 General Summary 158

$\begin{array}{llr}5.2 & \text { Future Studies } & 164\end{array}$

Chapter Six Experimental

6.1 General Experimental 168

6.2 Experiments Described in Chapter Two 173

6.3 Experiments Described in Chapter Three 188

6.4 Experiments Described in Chapter Four 209

$\begin{array}{ll}\text { References } & 221\end{array}$ 


\section{Abbreviations}

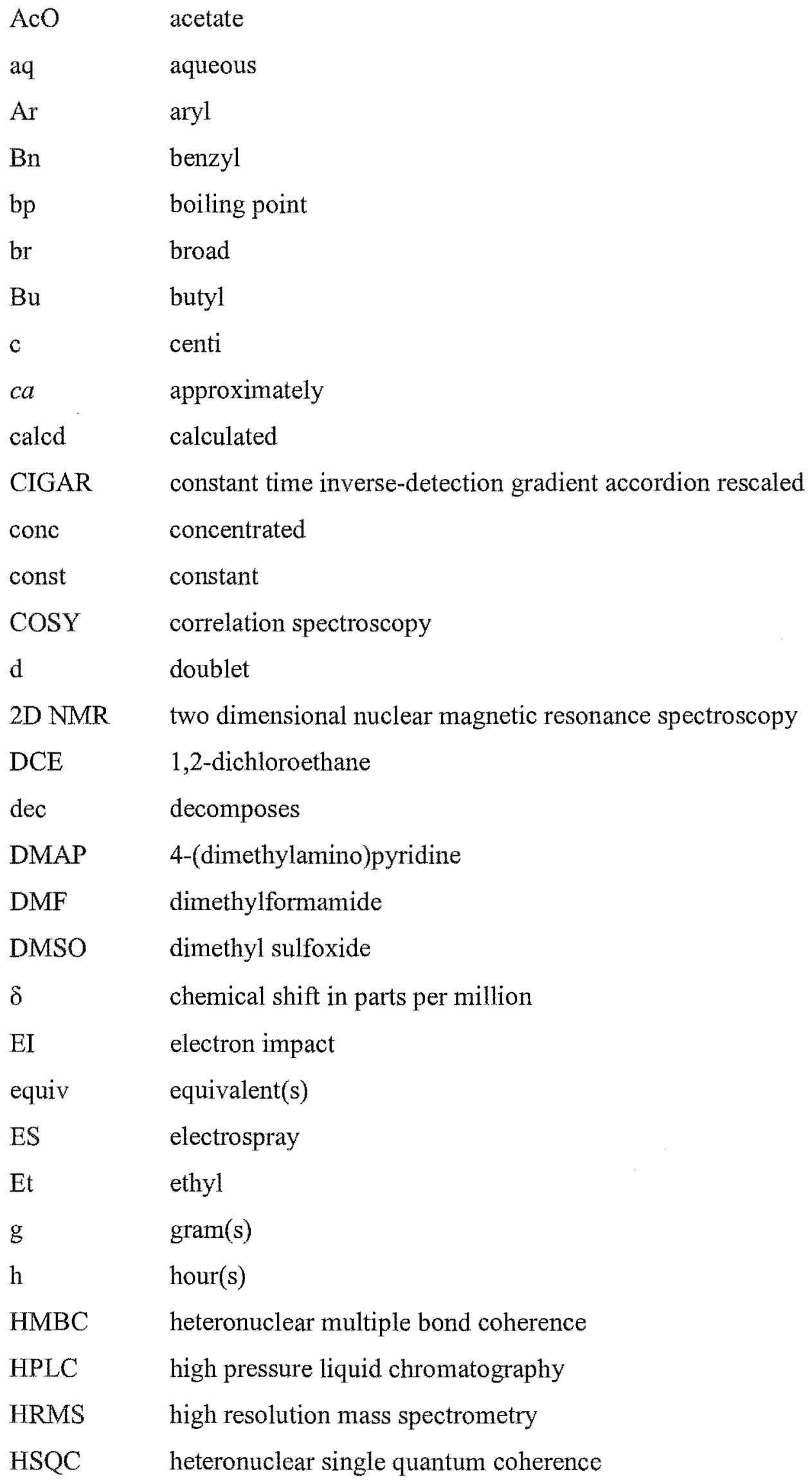


$\mathrm{Hz} \quad$ hertz

i-Pr isopropyl

IR infrared

$J \quad$ coupling constant

L litre(s)

LDA lithium diisopropylamide

LTMP lithium 2,2,6,6-tetramethylpiperidide

m metre(s), milli, multiplet

M mega, molar

mol mole(s)

$m$-CPBA meta-chloroperbenzoic acid

Me methyl

$\min \quad$ minute(s)

$\mathrm{mp} \quad$ melting point

MPLC medium pressure liquid chromatography

MS mass spectrometry

$m / z \quad$ mass to charge ratio

$\mu \quad$ micro

n- normal-

NOE nuclear Overhauser effect

NOESY nuclear Overhauser effect spectroscopy

NMR nuclear magnetic resonance spectroscopy

OAc acetate

$\mathrm{p} \quad$ page

para-

PMB para-methoxybenzyl

pp pages

ppm parts per million

rel relative

rt room temperature

s singlet

SAR structure-activity relationship

$\mathrm{t} \quad$ triplet

$t$ tertiary- 
TES triethylsilane

TFA trifluoroacetic acid

$\mathrm{TfOH} \quad$ triflic acid

THF tetrahydrofuran

TLC thin layer chromatography 


\section{Chapter One}

\section{Introduction}




\subsection{Marine Natural Products}

Natural products have traditionally played an important role in drug discovery. Over the last two hundred years, a diverse array of secondary metabolites numbering well into the thousands has been isolated from an equally diverse array of plant, animal and microbial sources. Some of these have found uses as biochemical tools for the elucidation of metabolic pathways, ${ }^{1}$ and many have been developed into medicinal agents for the treatment of disease. Numerous examples can be given. One of the earliest is that of morphine, from which many synthetic drugs are derived. ${ }^{2}$ Another striking example is the multitude of natural and semi-synthetic penicillins, which have transformed the treatment of bacterial infections in the last century. ${ }^{3}$

In contrast to terrestrially-derived natural products, the marine environment represents a relatively unexploited source of natural products. There are specific difficulties associated with the isolation and study of marine natural products. Not least of these is the difficulty of procuring significant quantities of the source organism from the marine environment. Such harvesting is costly and time-consuming and, as with any natural product isolation, has no guarantee of producing any useful leads for drug development. If a biologically active substance is discovered, it is usually isolated in only minute quantities - sometimes barely enough for complete characterisation, let alone drug development or supply.

Despite these difficulties, the field of marine natural products research has witnessed rapid and steady growth over the last thirty years. For example, in the period from 1965 to 1970 , around 100 natural products of marine origin were reported in the literature. This has dramatically risen to greater than 3,500 new reports in the period from 1991 to $1995{ }^{4}$ The later emergence of their chemistry compared to that of terrestrial natural products is due, in part, to the difficulty of access to marine organisms, and the relatively recent development of modern techniques (e.g. HPLC and 2D NMR) for the isolation and structure determination of complex, polar organic compounds, such as those often derived from marine sources.

There are many reasons for searching the oceans for new sources of biologically active compounds. For a start, water covers over two-thirds of the earth's surface and harbours tremendous biodiversity, most of which has yet to be investigated. Almost all of the major 
animal phyla are represented in the marine environment, while just over half occur on land. ${ }^{5}$ Second, the marine environment differs from the terrestrial environment markedly in salinity, temperature, pressure and light. It is logical, therefore, to expect different types of metabolites to be found in marine natural products compared with those from terrestrial sources. Indeed, it has been claimed that marine natural products are different in kind to those from terrestrial sources, both in shape ${ }^{5}$ and elemental composition. ${ }^{4}$ Statistical data reveal an abundance of marinederived metabolites containing nitrogen, which is commonly associated with a higher incidence of biological activity. ${ }^{4}$ Third, as many marine organisms are sessile, their survival frequently depends on chemical defense systems. There is widespread evidence indicating that secondary metabolites are more common in organisms without immune systems, such as marine invertebrates. ${ }^{6}$ These chemicals are often considerably more potent than those found on land, to counteract the high dilution of their aqueous environment.

As early as 1977, Faulkner suggested that marine organisms possess a greater prevalence of bioactive metabolites than do terrestrial organisms. ${ }^{7}$ A recent statistical analysis confirms that bioactive natural products from marine sources have increased at a greater rate than those from terrestrial sources. ${ }^{8}$ Many examples can be given to support this notion. Food poisonings due to the presence of certain toxins in shellfish and other seafoods are not uncommon and have received much media attention. Such events have aroused the attention of chemists and biologists alike, who were interested in discovering the source of these effects. Their investigations have uncovered some of the most structurally complex and toxic natural products known. For example, brevetoxin $B(\mathbf{1 . 1})^{9}$ is one of the agents responsible for the algal blooms, or "red tides", which have resulted in widespread killing of marine life, as well as human poisonings. Numerous marine-derived bioactive metabolites have since been discovered, whose biological effects are as diverse as their molecular structures (see Figure $\mathbf{1 . 1}$ for a selection).

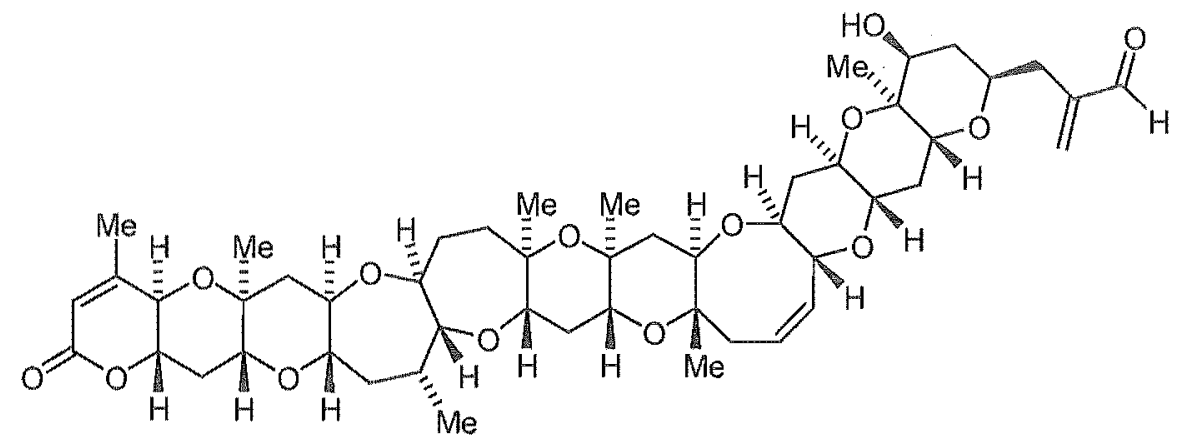




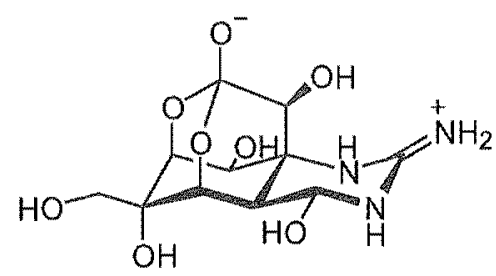

tetrodotoxin

toxic agent found in the puffer fish

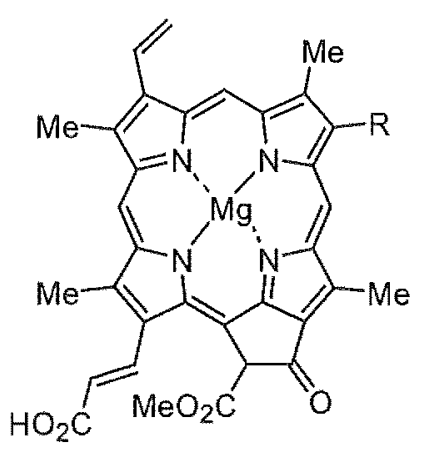

chlorophyll c photosynthetic pigment

$(\mathrm{R}=1: 1$ mixture of $\mathrm{C}_{2} \mathrm{H}_{5}$ and $\mathrm{C}_{2} \mathrm{H}_{3}$ )<smiles>Cn1c(N)c2ncn([C@@H]3O[C@H](CO)[C@@H](O)[C@H]3O)c2nc1=O</smiles>

1-methylisoguanosine causes muscle relaxation, hypotension, anti-inflammatory and antiallergic activity<smiles></smiles>

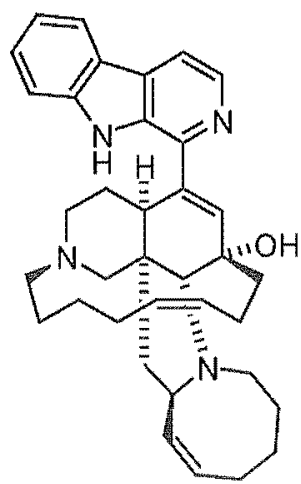

manzamine $A$ antitumour agent

\section{cephalostatin 1 \\ cephalostatin 1
antitumour agent} $\mathrm{NH}_{2}$

ATPase modulator

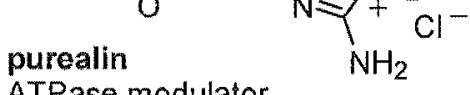

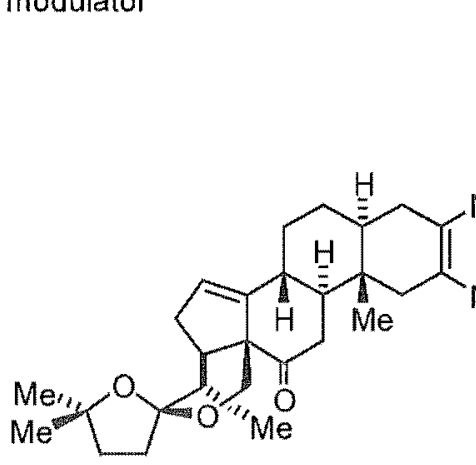


With the abundance of bioactive metabolites to have surfaced from the sea, one may expect that marine natural products would be well represented in the pool of drugs in current use. In actual fact, very few marine natural products have made it through to the commercial market. Thus far, the only contribution from the sea to cancer chemotherapy has been the discovery of the arabinoside nucleosides, which led to the development of cytosine arabinoside (ara-C, 1.2) which is used for the treatment of acute myeloblastic leukaemia. ${ }^{11}$ The reasons for this are many. Most significant is simply the later emergence of marine natural products chemistry compared with the chemistry of terrestrial natural products. Other reasons include problems of supply, which can leave potentially valuable compounds unexploited, and the extremely high toxicity of many marine natural products, which makes them of limited therapeutic value.

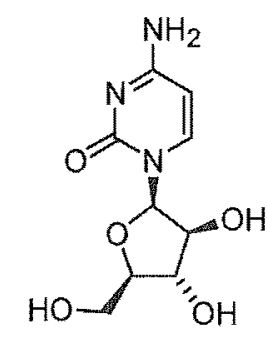

1.2

cytosine arabinoside

As with natural products in general, organic synthesis has played an important role in the field of marine natural products. As can be seen in Figure 1.1, marine natural products display great diversity in structure; consequently, they have stimulated the efforts of synthetic chemists worldwide. Traditionally, total synthesis has been important in confirming the proposed structure of a given compound. While modern techniques such as NMR spectroscopy have been very successful in allowing the structures of even very complex natural products to be determined, unambiguous structure determination cannot be achieved in every case. Often, uncertainty may exist regarding stereochemical issues, and the synthesis of one or more of the possible stereoisomers is required to establish the correct structure. And indeed, it has not been uncommon for a "total synthesis" to be achieved, only to find that the reported structure of the natural product was incorrect.

A recent example of this is seen in batzelladine $F(1.3)$, a bioactive marine alkaloid isolated from a Jamaican sponge in $1997 .^{12}$ This compound was of interest due to its ability to disrupt the 
processes involved in immunological responses by $\mathrm{T}$ lymphocytes. Such compounds may prove to be important in the understanding and treatment of autoimmune disorders. The originally proposed structure (1.4) was stated to have an anti relationship between the angular protons at C4 and C7. However, this was subsequently revised to syn, on the basis of synthetic studies on a model system. ${ }^{13}$ A number of stereochemical issues still remained undefined, namely, the configuration at $\mathrm{C} 16$ and the relative stereochemical relationship between the two tricyclic guanidine portions. This meant that there were eight compounds (four pairs of enantiomers) that fit the data for the proposed structure of batzelladine F. Where stereochemical elements are far removed from each other, as in this situation, their relative stereochemistries are inherently difficult to determine. Unless a crystal structure can be obtained, chemical synthesis is often the only way to reliably determine the relative and absolute stereochemistry. In an effort to define the exact structure of batzelladine F, Overman and Cohen synthesised one enantiomer of each of the four possible diastereomers. None of these, however, corresponded to the natural material and it was concluded that the proposed connectivity of batzelladine F was incorrect (compare the alkyl chains between the two structures). Re-examination of the spectroscopic data of the natural product suggested $\mathbf{1 . 3}$ as an alternative structure for batzelladine F. An enantioselective synthesis of 1.3 provided material which was identical with the natural product in all respects, and thus removed any uncertainty regarding the correct structure. ${ }^{14}$

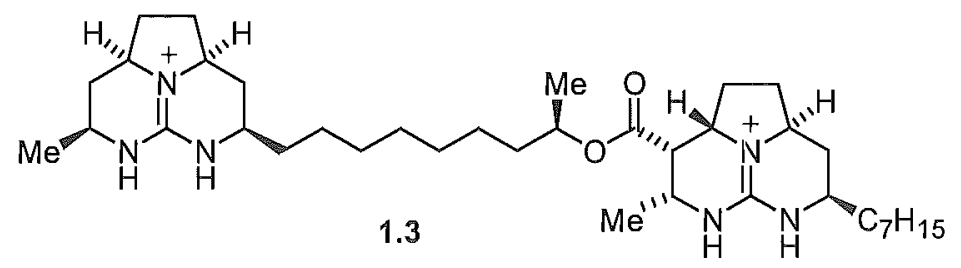

batzelladine $F$

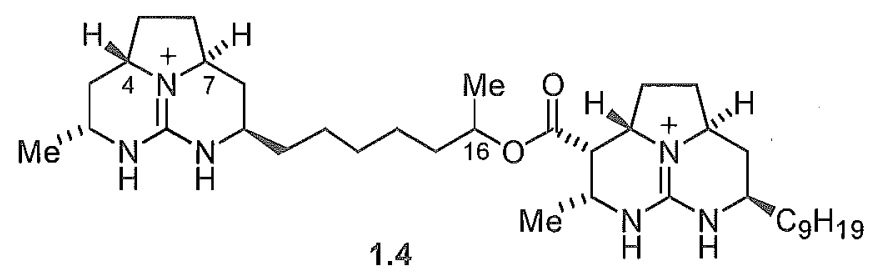

Another topical example of mis-assignment has very recently been reported. ${ }^{15}$ In this case, the compounds of interest were diazonamides A and B, two structurally novel marine natural products whose structures were reported to be 1.5 and 1.6 , respectively. ${ }^{16}$ These structures were 
proposed by comparison with a derivative of diazonamide $B$, whose structure was determined by $\mathrm{X}$-ray crystallography to be 1.7 .

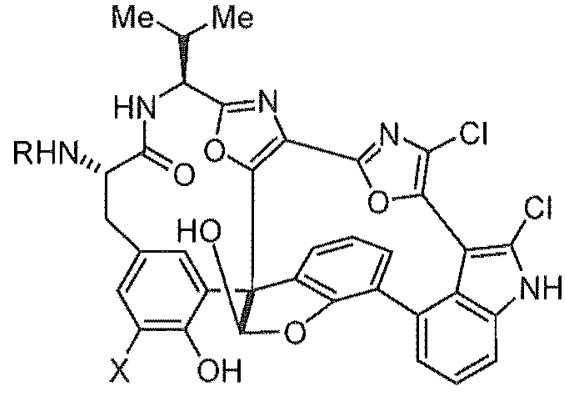

$1.5 X=H, R=5 \prod_{0}^{1 / 2 r} \mathrm{NH}_{2}$

$1.6 \mathrm{X}=\mathrm{Br}, \mathrm{R}=\mathrm{H}$

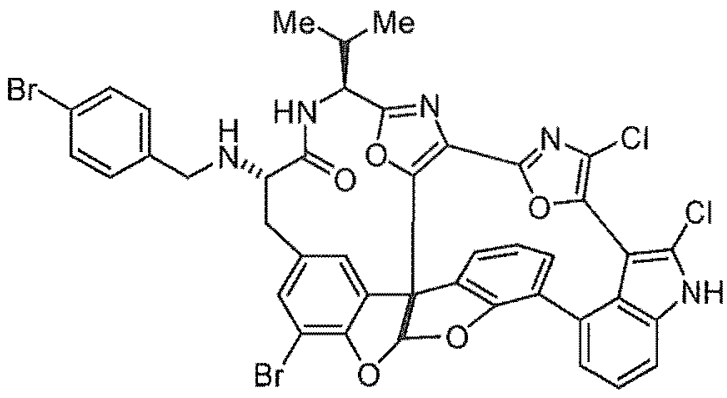

1.7

The extremely potent antitumour properties of diazonamide $\mathrm{A}$, combined with the novel architecture of these compounds, sparked considerable interest among synthetic chemists. ${ }^{17}$ Some ten years later, 1.5 - the structure originally proposed for diazonamide A - was produced by Harran et al. ${ }^{15 a}$ It was immediately apparent that neither the spectroscopic data, nor the chemical behaviour of 1.5 corresponded to those of diazonamide $A$. Furthermore, synthesis of 1.7, the reported derivative of diazonamide $B$ whose crystallographic characterisation formed the basis of the intial diazonamide assignments, gave a product which was different from the material derived from natural diazonamide $B$. It appeared that the crystallographic data of the diazonamide derivative had been misinterpreted. Further subtle discrepancies in the interpretation of mass spectral (MS) data were pointed out by Harran, and alternative structures for diazonamides $A$ and $B$-structures 1.8 and 1.9 respectively-were proposed based on the available data. ${ }^{15 b}$

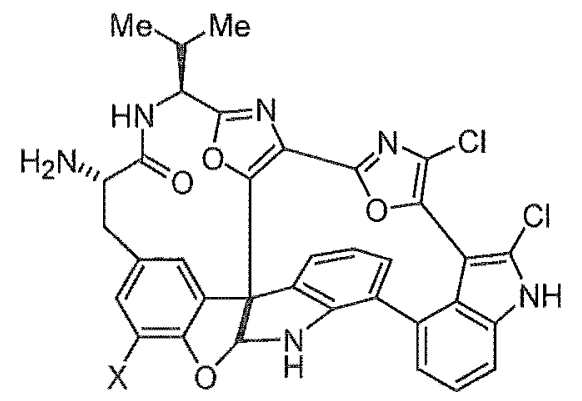<smiles></smiles>

$1.9 X=B r, R=H$ 
The examples of batzelladine $\mathrm{F}$ and the diazonamides highlight the importance of total synthesis in confirmation of structure-whether establishing unknown stereochemistry, or challenging incorrect connectivities - as even very reliable techniques are prone to mis-interpretation. In the diazonamide example, the work of Harran et al. also revealed a synthetic derivative $\mathbf{1 . 1 0}$ that displayed biological properties indistinguishable from those of natural diazonamide $\mathrm{A}^{15 \mathrm{~b}}$ This result is significant in that it may guide future work in the development of the diazonamides into medicinally useful agents.

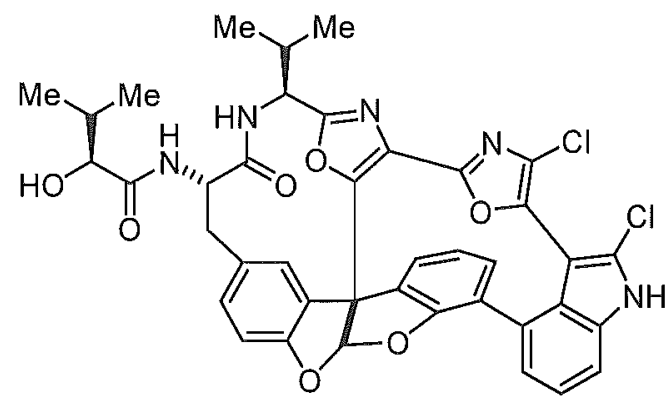

1.10

Chemical synthesis plays an equally important role in providing a supply of a natural product. This is no more evident than in the field of marine natural products chemistry. As has been mentioned, harvesting large quantities of marine organisms can be costly and time-consumingnot to mention the ecological impact of removing large amounts of a particular organism from the marine environment. In addition, secondary metabolites from marine organisms are usually produced in very low quantities and thus, large amounts of the organism are required to obtain a reasonable yield of the compound of interest.

A potent example of this is the marine natural product halichondrin B (1.11). This polyether macrolide was isolated from a sponge in Japanese waters, and shows extremely potent antitumour activity against both in vitro and in vivo human tumour models. ${ }^{18}$ However, halichondrin $\mathrm{B}$ is present in the natural source in only minute quantities and lack of material has prevented further investigation into its clinical potential. Accordingly, several groups have targeted their synthetic efforts towards halichondrin $B$, with the total synthesis of 1.11 being reported by Kishi's group in 1992. ${ }^{19}$ While a monumental effort, this synthesis does little to provide a practical supply of halichondrin $B$ as the process required a total of $\sim 120$ steps. However, improvements to the sequence have been made; the discovery that the right half diol 
1.12 exhibited similar activity to the natural product was of particular significance, as this intermediate could be generated in $\sim 70$ steps. $^{20}$ Further streamlining of this process may provide a useful source of a bioactive halichondrin-type compound, which could allow the development of the halichondrins and/or derivatives into medicinal drugs.
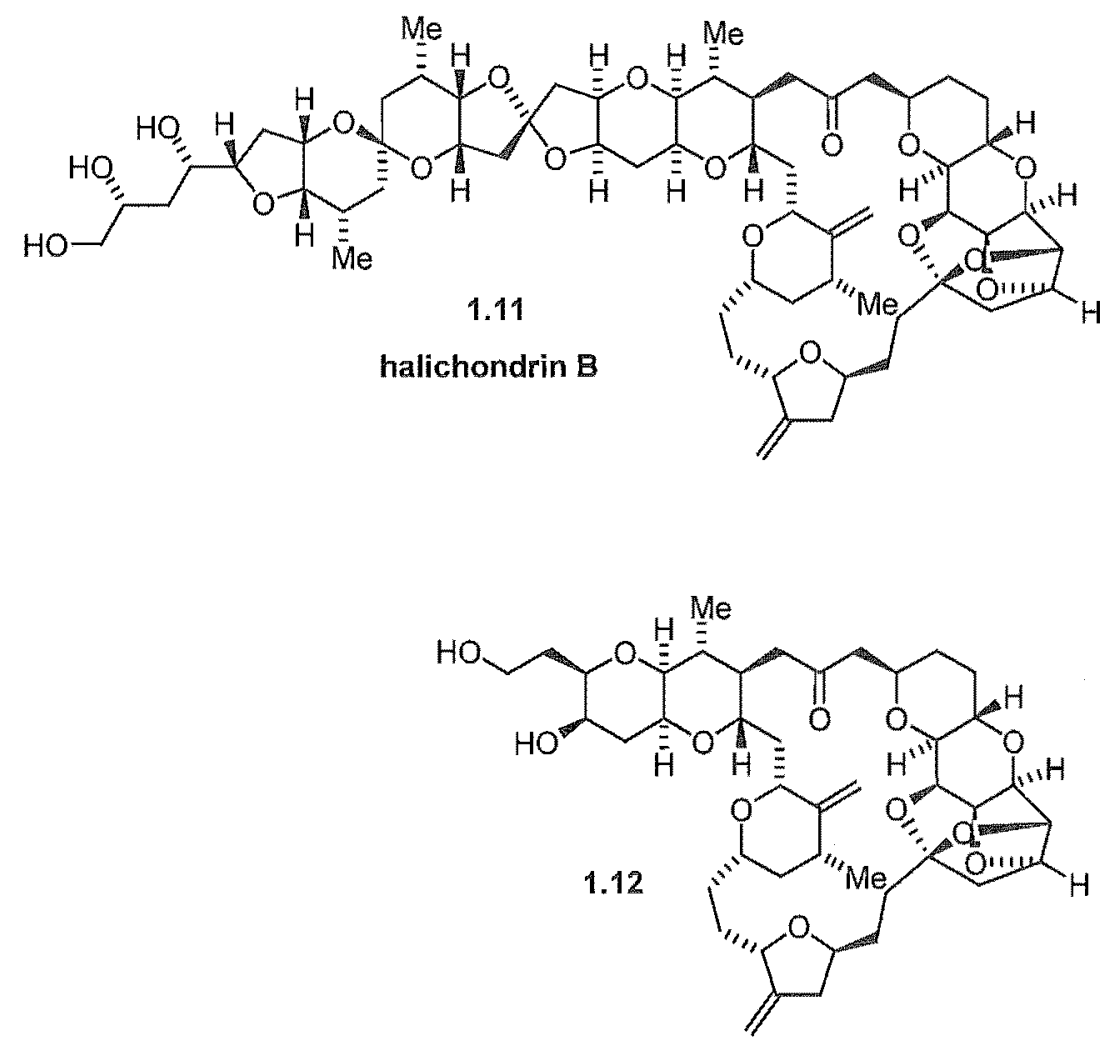

\subsubsection{The State-of-Play in Marine Natural Products}

Thirty years of research in marine natural products chemistry have resulted in numcrous discoveries of structurally novel compounds with great biological potential. It can now be said that the field is at a point where the pharmaceutical industry is on the verge of reaping the rewards of this work. Munro and coworkers list several compounds, or families of compounds, of marine origin which show strong potential as anticancer agents, some of which are currently in advanced stages of clinical trials. ${ }^{4}$ Of these, one of the most exciting is ecteinascidin (Et) 743 (1.13), which represents the state-of-play in marine natural products research today. Et 743 comprises a complex polycyclic framework with a high degree of oxygen and nitrogen functionality. Even though the colonial ascidian Ecteinascidia turbinata, from which it was isolated, ${ }^{21,22}$ was known to demonstrate potent antitumour properties since 1969 , fifteen years 
spanned the period between this discovery and the isolation of the active components. This interval reflects the relatively recent development of modern chromatographic techniques to enable the isolation of this very polar compound. The advent of fast atom bombardment mass spectrometry and 2D NMR techniques was also necessary for the structure of the ecteinascidins to be unravelled.

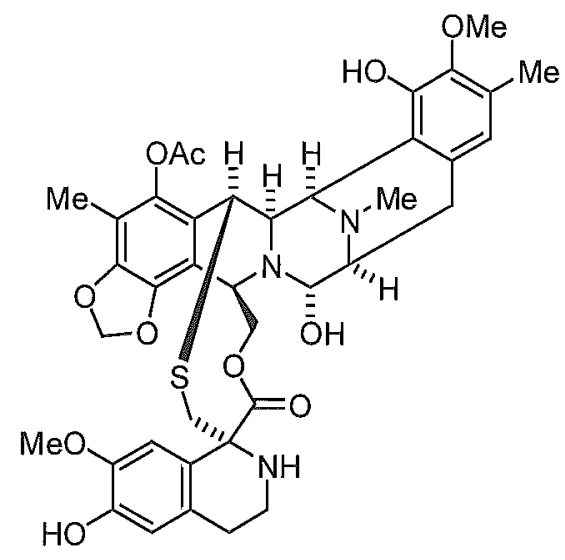

1.13

ecteinascidin 743

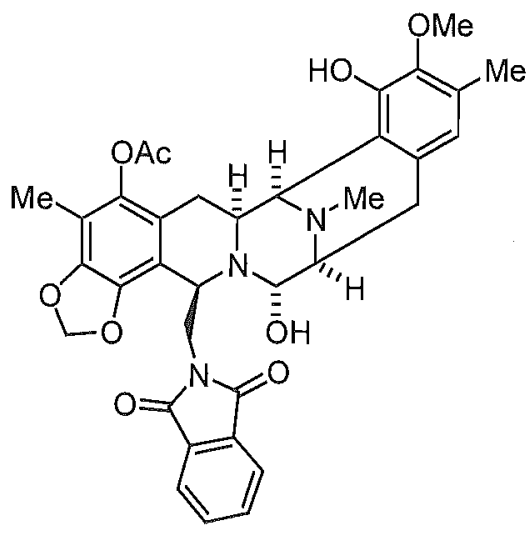

1.14

phthalascidin

Et 743 (1.13) was shown to possess potent cytotoxicity against a number of leukaemia cell lines $\left(\mathrm{IC}_{50}{ }^{*} 0.5 \mathrm{ng} / \mathrm{mL}\right.$ against L1210 leukaemia cells and $1.3 \mathrm{ng} / \mathrm{mL}$ against P388 murine leukaemia cells) and strong in vivo activity against murine and human tumour models (i.e. human tumours growing in athymic mice). In fact, Et 743 is 200 times more potent than the well-known and much-touted Taxol ${ }^{\circledR 2}{ }^{23}$ These findings have seen Et 743 advance into phase I clinical trials in 1996 and more recently, on the basis of promising results, into phase II clinical trials. A newdrug application is soon to be filed with the European Agency for the Evaluation of Medicinal Products for the treatment of advanced refractory sarcoma. ${ }^{24}$ Concurrent with clinical trials, investigation into its mode of action has been an active area of research for several years, culminating in the recent discovery that it acts by disruption of the cell's natural nucleotide excision-repair system. ${ }^{25,26}$ An improved mechanistic understanding of Et 743's role may lead to the rational design of new analogues, with greater potency and/or more desirable pharmacological properties.

\footnotetext{
${ }^{*} \mathrm{IC}_{50}$ is the concentration required to inhibit the growth of a given cell line by $50 \%$.
} 
Comprehensive investigation of any compound as a potential drug relies on a sustainable source of the compound. Despite being the most abundant of the ecsteinascidins, Et 743 was isolated in a yield of only $0.0001 \%$ from its ascidian source. While aquaculture of the ascidian is a possible solution to this problem, ${ }^{23,27} 1,000 \mathrm{~kg}$ of the organism would be required to produce just one gram of Et 743.

As such, efforts have been directed towards the synthesis of the ecteinascidins by a number of groups. ${ }^{28}$ In particular, a total synthesis of Et 743 was achieved by Corey's group in 1996, ${ }^{28 b}$ which provided a supply of the drug for industrial development. A consequence of this work was the discovery that an analogue of Et 743, phthalascidin (1.14), has similar in vitro activity and mode of action to Et 743 (1.13), but has the advantages of a more straightforward synthesis and greater stability in solution. ${ }^{29}$ Phthalascidin may become an important compound in the development of the ecteinascidins into a commercially available drug. Further improvements have been made to the syntheses of both 1.13 and 1.14 , resulting in a more reliable and scalable process. $^{30}$

From a commercial point of view, however, an even more attractive route has been revealed (Figure 1.2). ${ }^{31}$ Developed in the laboratories of Pharma Mar-a Spanish company which is carrying out the pharmaceutical development of Et 743 - this process employs cyanosafracin B (1.15) as the starting material, which is conveniently obtained on a kilogram scale through fermentation of the bacteria Pseudomonas fluorescens. A 22-step sequence is used to convert 1.15 into Et 743 . This process now provides a supply of Et 743 , in multigram quantities, for

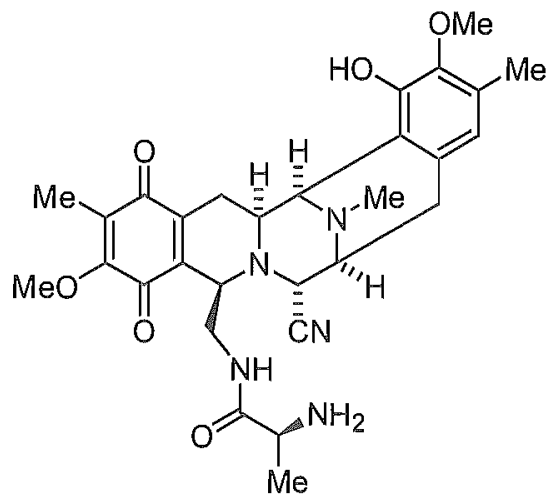

Et $743(1.13)$

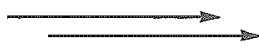

or

phthalascidin (1.14)

1.15

Figure 1.2 Semi-synthesis of Et 743 and phthalascidin from cyanosafracin B (1.15). 
further industrial development. In addition, numerous synthetic derivatives should allow for the preparation of a range of ecteinascidin analogues. Researchers in the field are confident that Et 743 will make a valuable contribution to cancer chemotherapy in the near future.

Et 743 stands at the forefront of research in the field of marine natural products. It highlights some of the difficulties associated with the study of chemicals from marine sources, such as the isolation of very polar compounds in only minute quantities and their development into commercially viable drugs. Yet it is clear that, with the current technology, solutions to these problems are available. The acute potency of Et 743, and other marine metabolites currently under evaluation in clinical trials, validate marine natural products research as a worthy endeavour.

The oceans remain largely unexplored and doubtless hold many secrets yet to be uncovered. Such a great source of biodiversity should continue to provide new lead compounds, which are needed to complement the existing arsenal of drugs. Many prevalent diseases have, as yet, no available remedies while few successful treatments in use today are totally effective or without undesirable side effects. Combine this with the growing problem of drug-resistance, and it is clear that chemists and biologists alike need to search for new and improved methods to combat the host of diseases and ailments which plague society, including cancer in its various forms, AIDS and multi-drug resistant pathogens. 


\subsection{The Variolins: a Unique Class of Marime Alkalloids}

\subsubsection{Introduction}

In 1994, Blunt, Munro and coworkers reported the P388 bioassay-guided isolation of a new class of marine alkaloids from the Antarctic sponge Kirkpatrica varialosa. ${ }^{32}$ The variolins (1.161.19) all possess a fused tricyclic heteroaromatic core-formally a pyrido[ $\left.3{ }^{\prime}, 2^{\prime}: 4,5\right]$ pyrrolo[1,2c]pyrimidine bearing a heterocyclic substituent at C5 in the cases of variolins A (1.16) and B (1.17) and $N\left(3^{\prime}\right)$-methyl tetrahydrovariolin $\mathrm{B}(1.18)$. A fourth compound, named variolin $\mathrm{D}$ (1.19), bears an ester grouping at $\mathrm{C} 5$, and is thought to be a degradation product produced by aerial oxidation of the variolins during the extraction process. The pyrido[3',2':4,5]pyrrolo[1,2clpyrimidine ring system has not been encountered in any other natural products. Prior to the isolation of the variolins, the tricyclic system had been described only once (as synthetic derivatives), albeit in a different oxidation state to the variolin core. ${ }^{33}$

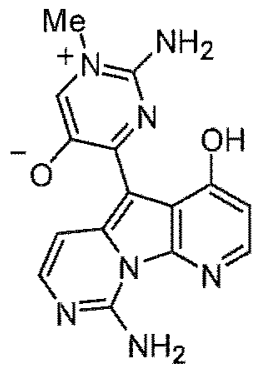

1.16

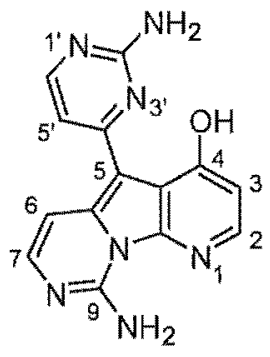

1.17

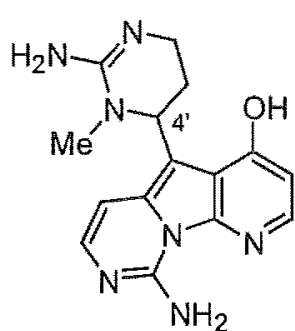

1.18<smiles>COC(=O)c1c2c(O)ccnc2n2c(N)nccc12</smiles>

1.19

variolin $\mathrm{B}$ variolin $\mathrm{D}$

\subsubsection{Structure Elucidation}

Despite their modest size, the variolins presented quite a challenge in their structural elucidation. This was largely due to the presence of several ring-nitrogen atoms and relatively few hydrogen atoms in the structure, which makes $\mathrm{C}-\mathrm{C}$ connectivities difficult to establish using long-range NMR techniques. While extensive NMR analysis (HMQC, HMBC, NOE) was carried out, this did not result in definitive structures being assigned to the variolins. Resorting to other methods, 
very small crystals of variolin $B$ were obtained from a TFA/water solution, which could be studied by rotating-anode X-ray diffraction. This resulted in structure 1.17 being assigned to variolin $\mathrm{B}^{32 \mathrm{a}}$

Once the structure of variolin B (11.17) had been established with absolute certainty, the wealth of NMR data that had been collected could be interpreted. Of particular interest are the exchangeable protons of the amino and hydroxyl groups. Two broad signals at $\delta 8.6$ and 9.9 were assigned to the protons of the same $\mathrm{NH}_{2}$ group at C9. These assignments were supported by the fact that irradiation of either of the signals led to the disappearance of the other, and that when the spectrum was run at higher temperatures the two signals collapsed into one. The difference in chemical shifts suggests that bond-rotation around the $\mathrm{C} 9-\mathrm{NH}_{2}$ bond is somewhat restricted, presumably due to hydrogen bonding between one of the protons and N1. This is supported by the crystal structure data, which shows that the two atoms lie $2.063 \AA$ apart. $\pi$ Overlap of the nitrogen-lone pair with the fused pyrimidine ring may also play a role in restricting rotation of the amino group. The other $\mathrm{NH}_{2}$ group displayed no such separation of signals, being represented by a broad signal at $\delta 7.1$ integrating for two protons. The remaining exchangeable proton, bound to the oxygen at $\mathrm{C} 4$, gave rise to a broad signal at $\delta 16.1$. Its extremely deshielded nature suggests hydrogen bonding, presumably to N3' of the pendant pyrimidine ring. In the solid state, this is indeed the case, with the two atoms lying $2.687 \AA$ apart. This hydrogen bonding has the effect of drawing the 2-aminopyrimidine ring close to the plane of the tricyclic core. This is observed in both the crystal structure, in which the 2 aminopyrimidine ring is $23.8^{\circ}$ out of the pyridopyrrolopyrimidine mean plane, and in the NOE experiments which displayed a strong correlation between $\mathrm{H} 6$ of the pyridopyrrolopyrimidine moiety and $\mathrm{H}^{\prime}$ ' of the 2-aminopyrimidine ring. The structure of variolin D (1.19) was determined by comparison to the spectral data of variolin $B$, and also by chemical transformation from variolin $\mathrm{A}^{32 \mathrm{a}}$

As with variolin B, X-ray crystallography was required to establish the structure of variolin A $(1.16) .{ }^{32 b}$ This, in itself, was no mean feat as it was found to be extremely difficult to obtain suitable crystals for diffraction studies. Finally, a TFA/water solution yielded a few small needles of variolin $A$, whose structure was determined to be that of 1.16. This allowed interpretation of the NMR data, resulting in the complete assignment of all the ${ }^{1} \mathrm{H}$ and ${ }^{13} \mathrm{C}$ signals. 
The NMR analysis of $N\left(3^{\prime}\right)$-methyl tetrahydrovariolin B (1.18) was complicated by the existence of axial and equatorial conformers at $\mathrm{C}^{\prime}{ }^{32 \mathrm{~b}}$ The natural product was, therefore, allowed to react with an excess of methyl iodide, to give hexamethyl derivative 1.20 whose structure could be solved by NMR. The results of the NMR analysis were then extrapolated to $N\left(3^{\prime}\right)$-methyl tetrahydrovariolin B, enabling a satisfactory structure to be proposed.

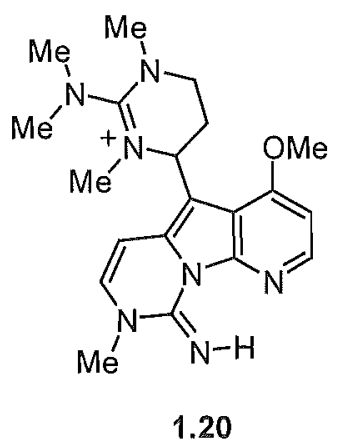

\subsubsection{Biological Activity}

The variolins were tested for biological activity against a range of cell lines and microorganisms. In the P388 (murine leukaemia cell line) assay, variolin B (1.17) was the most active with an $\mathrm{IC}_{50}$ of $210 \mathrm{ng} / \mathrm{mL}^{32 \mathrm{a}}$ while variolin $\mathrm{A}(1.16)$ displayed only weak activity $\left(\mathrm{IC}_{50} 3,800\right.$ $\mathrm{ng} / \mathrm{mL}){ }^{32 \mathrm{~b}} N\left(3^{\prime}\right)$-Methyl tetrahydrovariolin $\mathrm{B}(1 \mathbf{1 8})$ was tested in vivo against $\mathrm{P} 388$ murine leukaemia, where it prolonged lifespan by $25 \%$ at a concentration of $10 \mathrm{mg} / \mathrm{kg}^{32 \mathrm{~b}}$

$N\left(3^{\prime}\right)$-Methyl tetrahydrovariolin B was also tested against the HCT 116 (human colon carcinoma) cell line, having an $\mathrm{IC}_{50}$ of $480 \mathrm{ng} / \mathrm{mL}$. Anti-bacterial activity was observed in $\mathbf{1 . 1 8}$ in its inhibition of the growth of Saccharomyces cerevisiae $(36 \mathrm{~mm}$ zone at $2 \mathrm{mg} / \mathrm{mL}) .{ }^{32 \mathrm{~b}}$ Variolin B (1.17) was inactive against the range of bacteria and fungi, although in anti-viral assays it was found to be four times more effective against Herpes simplex than against the Polio virus. ${ }^{32 a}$ Variolin D (1.19) was inactive in all the assays, leading to the suggestion that substitution with the 2-aminopyrimidine ring at $\mathrm{C} 5$ was necessary for biological activity. ${ }^{32 \mathrm{a}}$

In the late 1990s, Pharma Mar (the same company which is developing Et 743) became interested in variolin B (1.17) - having the most potent anticancer properties of the variolins- 
as a new lead compound. However, due to the isolated origin of the sponge, a sustainable supply of variolin B was not available. Aside from the cost and difficulties of accessing the sponge $K$. varialosa from Antarctica, the 1991 Madrid Protocol to the Antarctic Treaty prohibits any person from removing flora or fauna from the region without the authorisation of their government. ${ }^{34}$

As a result of these findings, variolin B (1.17) has emerged as an attractive synthetic target. Over the last three years, a flurry of reports has appeared describing syntheses of either fragments of the variolin core, ${ }^{35}$ or the intact core with substituents attached at various positions. ${ }^{36,37,38}$ This body of work, from several groups, has been carried out concurrently with our own efforts. ${ }^{39}$

To put the work described in this thesis into perspective, a summary of related synthetic workalthough not directed specifically towards the variolins-is given below, followed by an account of the successful syntheses of the variolin core that have appeared up until the present time.

\subsubsection{Related Synthetic Work}

An examination of the literature (pre-1999) for the pyrido[3',2':4,5]pyrrolo[1,2-c]pyrimidine core found in the variolins revealed just two reports of ring systems which were closely related to the variolin core. ${ }^{33,40}$

In the first of these reports, ${ }^{33}$ the trimethyl-substituted $3 H-7$-azaindole ${ }^{*} 1.21$ was reacted with either benzoyl isothiocyanate or ethoxycarbonyl isothiocyanate to give 1.22 and 1.23 , respectively (Scheme 1.1). Heating in pyridine led to the elimination of water or ethanol, respectively, to give the cyclised products $\mathbf{1 . 2 4}$ and 1.25 in excellent yields. It should be noted that the cycloadducts differ from the variolin core in both oxidation state and the nature and position of the substituents.

\footnotetext{
"The term 7-azaindole is used throughout this thesis for practicality, rather than the less convenient pyrrolo[2,3b]pyridine.
} 
<smiles>CC(C)(C)c1cccnc1N/C(=C\C(=S)NC(=O)c1ccccc1)C(=O)O</smiles>

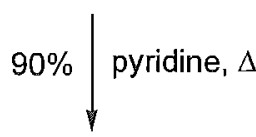<smiles>CC1(C)c2cccnc2-n2c1cc(=S)nc2-c1ccccc1</smiles>

1.24<smiles>CCOC(=O)NC=CCc1ccccc1C</smiles><smiles>CCOC(=O)NC(=S)/C=C1\Nc2ncccc2C1(C)C</smiles>

1.23 $95 \% \downarrow$ pyridine, $\Delta$<smiles>CC1(C)c2cccnc2-n2c1cc(=S)[nH]c2=O</smiles>

1.25

\section{Scheme 1.1}

The later publication ${ }^{40}$ reports the (serendipitous) synthesis of the pyrido[3',2':4,5]pyrrolo[1,2c]pyrimidine moiety as part of a larger fused system (Scheme 1.2). Mérour and coworkers formed the 2-lithio-7-azaindole 1.26, using the phenylsulfonyl group to direct metalation to $\mathrm{C} 2$, and quenched the lithio-species with a range of electrophiles. When tributylstannyl chloride was used, the 2-stannyl derivative 1.27 was obtained. This underwent Pd-catalysed cross-coupling with the heteroaromatic iodide $\mathbf{1 . 2 8}$ to give $\mathbf{1 . 2 9}$. At this stage, they sought to cyclise $\mathbf{1 . 2 9}$ to the azaindole 3-position under Bischler-Napieralski conditions. However, rather than the expected reaction at $\mathrm{C} 3$, an unexpected dephenylsulfonylation led to a preferred N1 attack on the amide to give, after in situ chlorination, the variolin-like product $\mathbf{1 . 3 0}$, isolated in $40 \%$ yield. 


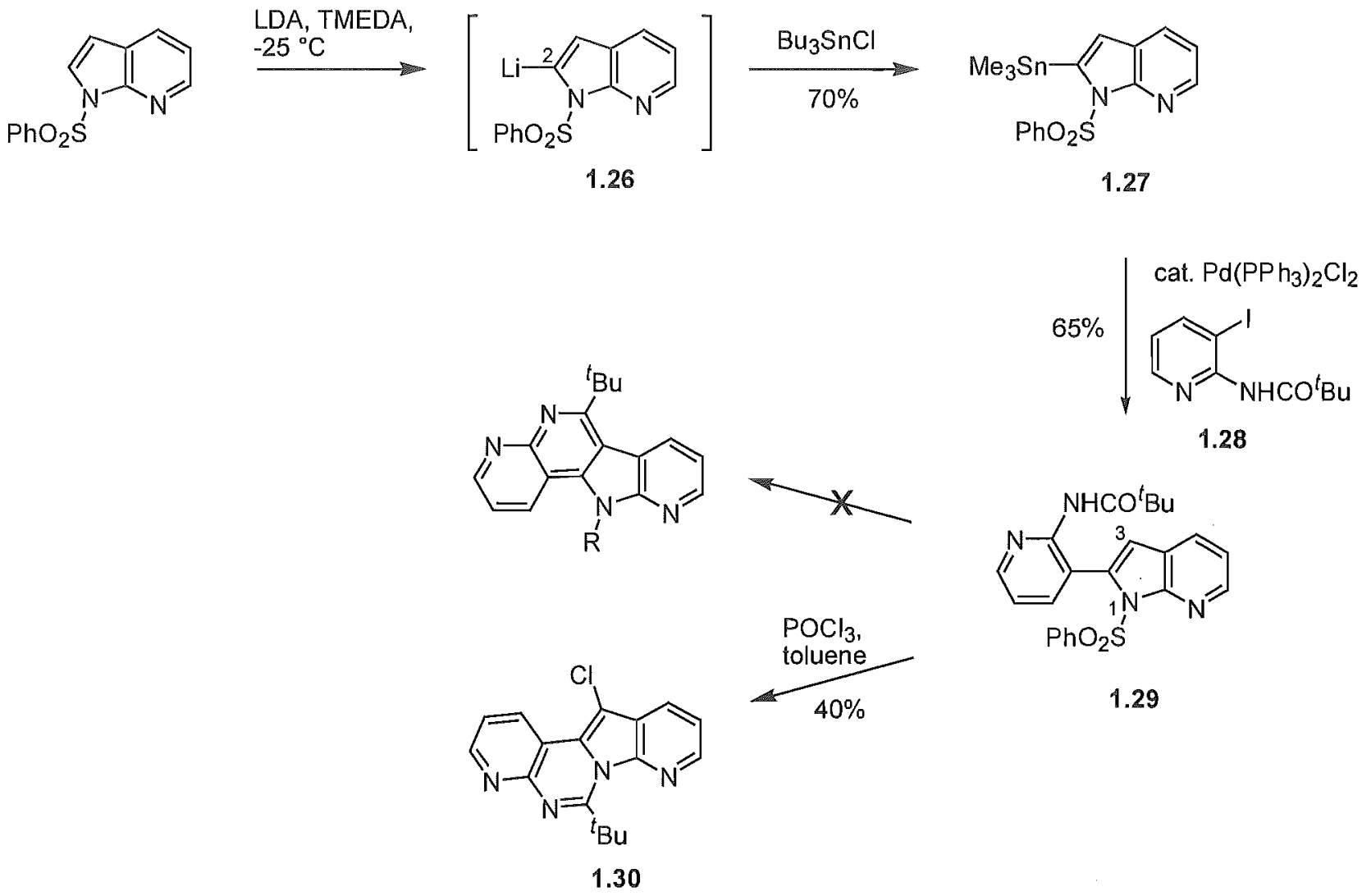

Scheme 1.2

While not specifically directed towards the variolins, both of the aforementioned schemes do represent a potential approach to the variolin core: an appropriate 2-substituted 7-azaindole is cyclised to the indole nitrogen (i.e. N1 of the azaindole) to give the variolin core (or a partially hydrogenated version, thereof). This can be viewed retrosynthetically as a disconnection between C9 and N10 of the variolin core (Figure 1.3).

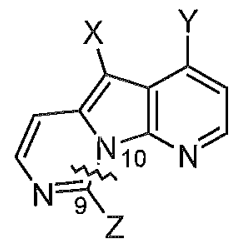

Figure 1.3 Disconnection of the C9-N10 bond of the variolin core. 
If such a strategy was applied to a synthesis of the variolins, the general requirements would be:

a) an efficient route to a 4-substituted 7-azaindole;

b) a means of introducing a side chain at $\mathrm{C} 2$ (such as the lithiation/transmetalation and Pdcatalysed cross-coupling chemistry demonstrated by Mérour); alternatively, an appropriate handle for functionalisation at $\mathrm{C} 2$ may be included in the construction of the azaindole system;

c) a means of cyclising the $\mathrm{C} 2$ side chain to the indole nitrogen to give the pyrido $\left[3^{\prime}, 2^{\prime}: 4,5\right]$ pyrrolo[1,2-c]pyrimidine core at the correct level of oxidation;

d) an appropriate method of introducing the amino group (or a suitable equivalent) at C9 of the variolin core;

e) either a reactive group at C5 of the variolin core to allow coupling of an intact pyrimidine heterocycle, or a carbon substituent upon which, the 2-aminopyrimidine ring can be built up.

Clearly, the first synthesis (Scheme 1.1) is not suited to the variolins as it does not provide the functionality at C5 of the variolin core structure, which is required for the introduction or construction of the 2-aminopyrimidine ring. Mérour's work does (fortuitously) leave a chlorine at $\mathrm{C} 5$, however, it is doubtful whether this would undergo oxidative addition with palladium. A further point to consider from this work is that the Pd-catalysed coupling of stannane 1.27 was attempted with a very limited range of iodides. When vinyl iodide 1.31 was used, no coupling product was formed, despite a variety of reaction conditions being investigated. Using $\mathrm{Pd}\left(\mathrm{PPh}_{3}\right)_{4}$ in refluxing toluene, the dimer of the vinyl species, 1.32 , was isolated in $30 \%$ yield. This casts a shadow on the use of this methodology for a synthesis of the variolins, which would require coupling of a vinyl iodide similar to $\mathbf{1 . 3 1}$.

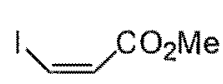

1.31

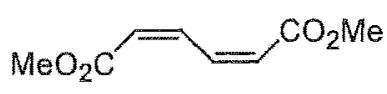

1.32 


\subsubsection{Symthetic Efforts Towards the Variolins}

As has been mentioned, a number of syntheses of the variolin core have recently been reported by three separate groups - all based in Spain. All three syntheses employ the strategy described above, i.e. disconnection between $\mathrm{C} 9$ and N10, leading to a 2-substituted 7-azaindole.

\subsubsection{Fresneda and Molina's Approach to the Variolin Core and Synthesis of Variolin B}

Fresneda, Molina and coworkers were the first to report their synthesis of the pyrido[3', $\left.2^{\prime}: 4,5\right]$ pyrrolo[1,2-c]pyrimidine core, which is shown in Scheme 1.3. ${ }^{36 a}$ Their approach began using 3-formyl-4-methoxypyridine (1.33), available in one step from 4methoxypyridine. ${ }^{41}$ Condensation with ethyl azidoacetate gave the vinyl azide 1.34 , which was cyclised via a nitrene insertion process to produce the 2 -substituted azaindole 1.35 . This is a modification of the Hemetsberger indole synthesis. ${ }^{42}$ The side-chain was extended through a four-step sequence (again, employing ethyl azidoacetate) to give the protected vinylazide 1.36. This underwent the Staudinger reaction with triphenylphosphine to give (after deprotection of the SEM group) the iminophosphorane 1.37, which was set up for ring-closure to the variolin core 1.38. ${ }^{43}$ This was effected by treatment with several dielectrophilic isocyanates (or ethoxycarbonyl isothiocyanate), in excellent yields.<smiles>COc1ccncc1C=O</smiles>

1.33

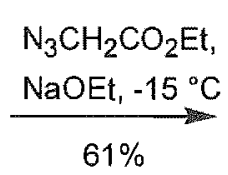

$61 \%$<smiles>CCOC(=O)/C(N)=C/c1cnccc1OC</smiles>

1.34

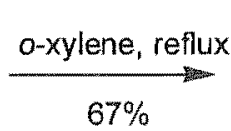

$67 \%$<smiles>CCOC(=O)c1cc2c(OC)ccnc2[nH]1</smiles>

1.35<smiles>[R]Nc1nc(C(=O)OCC)cc2cc3c(OC)ccnc3n12</smiles>

1.38

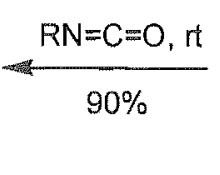

$0 \%$<smiles>CCOC(=O)/C(=C/c1cc2c(OC)ccnc2[nH]1)N=P</smiles>

1.37

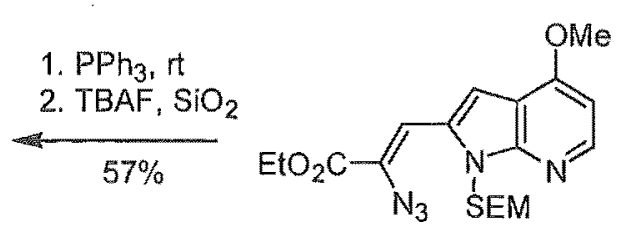

1.36

$\mathrm{SEM}=$ hO $\mathrm{OSIME}_{3}$ 
Fresneda and Molina's ten-step synthesis of the variolin core successfully introduces the oxygen and nitrogen functionalities at $\mathrm{C} 4$ and $\mathrm{C} 9$ respectively, as required by all of the variolins. However, it leaves unaddressed the issue of the superfluous ethoxycarbonyl group at C7. Neither was any mention made as to how the pyrimidine heterocycle might be introduced at C5.

In January 2002, while this dissertation was being prepared, these workers reported their progress in regard to both of these matters. This work has culminated in a total synthesis of variolin $\mathrm{B}^{36 \mathrm{~b}}$ The construction of the pyrimidine heterocycle was based on methodology they had previously used for a series of related natural products, meridianins C-E (1.39$1.41)^{35 d, 36 a, 44}$

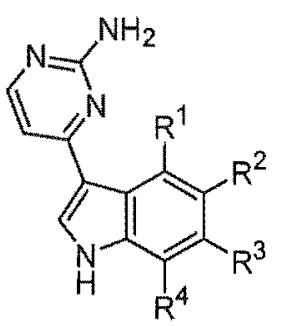

$$
\begin{array}{ll}
\text { meridianins } & C(1.39) R^{1}, R^{3}, R^{4}=H, R^{2}=B r \\
& D(1.40) R^{1}, R^{2}, R^{4}=H, R^{3}=B r \\
& E(1.41) R^{1}=O H, R^{2}, R^{3}=H, R^{4}=B r
\end{array}
$$

Functionalisation at $\mathrm{C} 5$ of the variolin core was achieved in a highly efficient manner by reaction with bromine in pyridine to give 1.42 (Scheme 1.4). This regioselectivity observed in the pyrido[3', $\left.2^{\prime}: 4,5\right]$ pyrrolo[1,2-c]pyrimidine system is analogous to the behaviour of indoles with electrophiles, which generally react at $\mathrm{C} 3$ of the 5-membered ring. Pd-catalysed coupling with ( $\alpha$-ethoxyvinyl)trimethylstannane gave ketone 1.43 , which was extended by treatment with dimethylformamide di-t-butyl acetal to give 1.44. The 2-aminopyrimidine ring was closed by heating with guanidine hydrochloride and anhydrous $\mathrm{K}_{2} \mathrm{CO}_{3}$. Under these conditions, hydrolysis of the ester occurred concurrently, giving 1.45 in $89 \%$ yield. Decarboxylation of 1.45 proved to be rather difficult. Similar difficulties were encountered by another Spanish group when attempting to decarboxylate a related fused pyrimidine derivative. ${ }^{35 a}$ Eventually, it was found that decarboxylation and simultaneous deprotection of the methoxy group could be accomplished in $35 \%$ yield by heating at $260^{\circ} \mathrm{C}$ in diphenyl ether, to give 1.46 . The final step involved treatment of $\mathbf{1 . 4 6}$ with triflic acid at room temperature to remove the $\alpha$-methylbenzyl protecting group, giving the natural product variolin B (1.17) in $90 \%$ yield. 


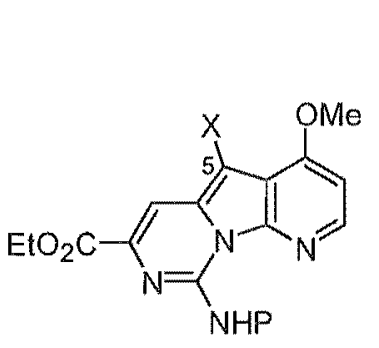

$$
\begin{aligned}
& \text { pyridine, } \\
& \mathrm{Br}_{2}, \mathrm{O}^{\circ} \mathrm{C} \\
& 93 \%
\end{aligned}\left[\begin{array}{l}
1.38 \mathrm{X}=\mathrm{H} \\
-1.42 \mathrm{X}=\mathrm{Br}
\end{array}\right.
$$<smiles></smiles>

1.17

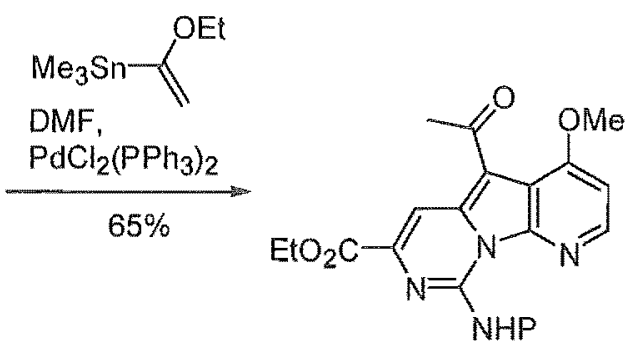

1.43

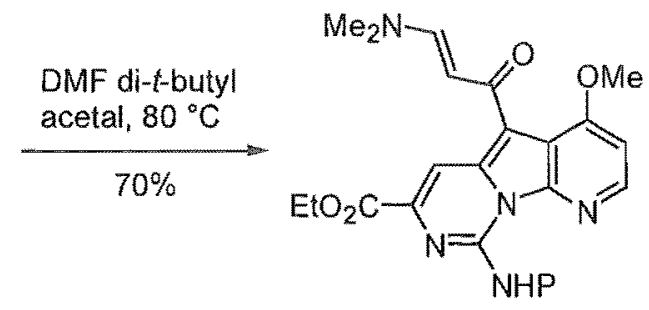

1.44

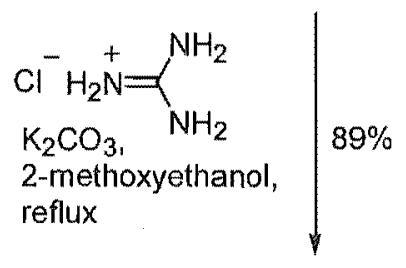

$\frac{\text { triflic acid, } n}{90 \%}$<smiles></smiles>

1.46<smiles>COc1ccnc2c1c(-c1ccnc(N)n1)c1cc(C(=O)O)nc(N)n12</smiles>

1.45

variolin $\mathbf{B}$

Scheme 1.4

This synthesis should be amenable to the production of analogues, varying in the substituents at both $\mathrm{C} 5$ and $\mathrm{C} 9$ of the variolin core. However, the linear nature of this synthesis does draw it out somewhat, the variolin core 1.38 requiring ten steps for its synthesis, while a further six steps were necessary to convert this to the natural product. The overall yield for the sequence was $1.2 \%$. 


\subsubsection{Vaquero's Approach to the Variolin Core}

Shortly after the appearance of Fresneda and Molina's original communication, ${ }^{36 a}$ Vaquero's group published a four-step synthesis of the variolin core. In their approach, intact azaindoles were utilised as starting materials, with the variolin core being formed by annelation of a sidechain attached to $\mathrm{C} 2$ of the azaindole. Thus, azaindoles 1.47 were protected at the indole nitrogen, and dibrominated to give the bromomethyl compounds 1.48 (Scheme 1.5). These products incorporate the functionality required both at $\mathrm{C} 3$ (what would become $\mathrm{C} 5$ of the variolin core) and the $\mathrm{C} 2$ side chain. Extension of the bromomethyl group was achieved using tosylmethyl isocyanide under phase-transfer conditions. Unexpectedly, the product isolated from this reaction was, in fact, variolin core structure 1.49. This was presumed to have formed due to an intramolecular transfer of the $N$-protecting group, which enabled nucleophilic attack by the indole nitrogen on the isocyanide to produce 1.49 .

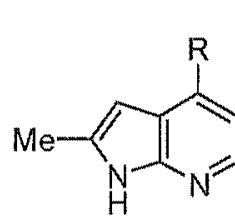

1.47

a $\mathrm{R}=\mathrm{H}$

b $\mathrm{R}=\mathrm{OMe}$

c $\mathrm{R}=\mathrm{Cl}$
1. $\mathrm{ClCO}_{2} \mathrm{Me}$

2. NBS, $\mathrm{CCl}_{4}$

3. $\mathrm{NBS}, \mathrm{CCl}_{4},\left(\mathrm{PhCO}_{2}\right)_{2}$

$82 \%$ for a $(R=H)$

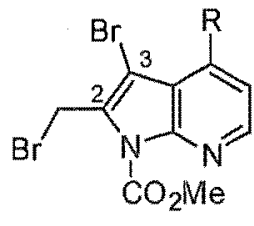

1.48

$\mathrm{CNCH}_{2} \mathrm{Ts}_{3}$ $\mathrm{NaOH}, \mathrm{CH}_{2} \mathrm{Cl}_{2}$<smiles>[R]c1ccnc2c1c(Br)c1cc(C(C)C)ncn12</smiles>

1.49

a $65 \%$

b $61 \%$

c $27 \%$

Scheme 1.5

Three examples were given, including one that possessed a methoxy group suitably positioned on the pyridine ring, which would serve as a synthetic equivalent for the hydroxyl group found in 
all the variolins. This certainly offers a much more direct route to the variolin core than Fresneda and Molina's ten-step sequence and should allow the introduction of a variety of heterocycles at C5. As with Fresneda and Molina's synthesis, however, the procedure suffers in that the methoxycarbonyl group is retained at C7. The procedure also requires introduction of the amino group at C9. However, to date, there has been no report on how this is to be accomplished.

\subsubsection{3 Álvarez and Joule's Synthesis of Deoxyvariolin $\mathbb{B}$}

The third synthesis of the variolin core, which appeared within approximately six months of Fresneda and Molina's original communication, was due to the group of Álvarez and Joule. ${ }^{38}$ In this paper, they elected to ignore the hydroxyl group found in the variolins, their goal being a synthesis of the deoxy-analogue of variolin $\mathrm{B}$, termed deoxyvariolin $\mathrm{B}(1.50)$. Their overall strategy involved initial formation of the variolin core, followed by selective functionalisation at C5 and introduction of an intact pyrimidine heterocycle.

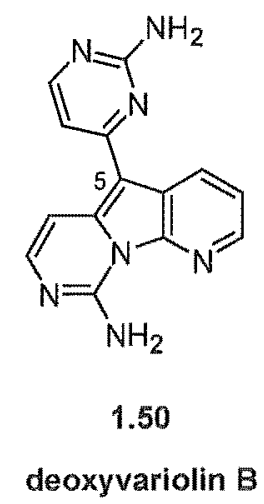

Their synthesis of the variolin core is portrayed in Scheme 1.6. Starting with commercially available 7-azaindole, Álvarez adapted Katritzky's method ${ }^{45}$ for lithiation of indoles at the 2position. This was found to be more convenient than the phenylsulfonyl-directed lithiation method described by Mérour ${ }^{40}$ and gave superior results. Thus, the lithio-species 1.51 with was quenched with $N$-pthalimidoaminoacetaldehyde to give the 2-substituted azaindole 1.52 . Following protection/deprotection steps, cyclisation to tetrahydropyrimidinone 1.53 was effected upon treatment with triphosgene and Hünig's base. Conversion to pyrimidinone 1.54 was readily achieved by hydrolysis of the protecting group and dehydration via the mesylate. 


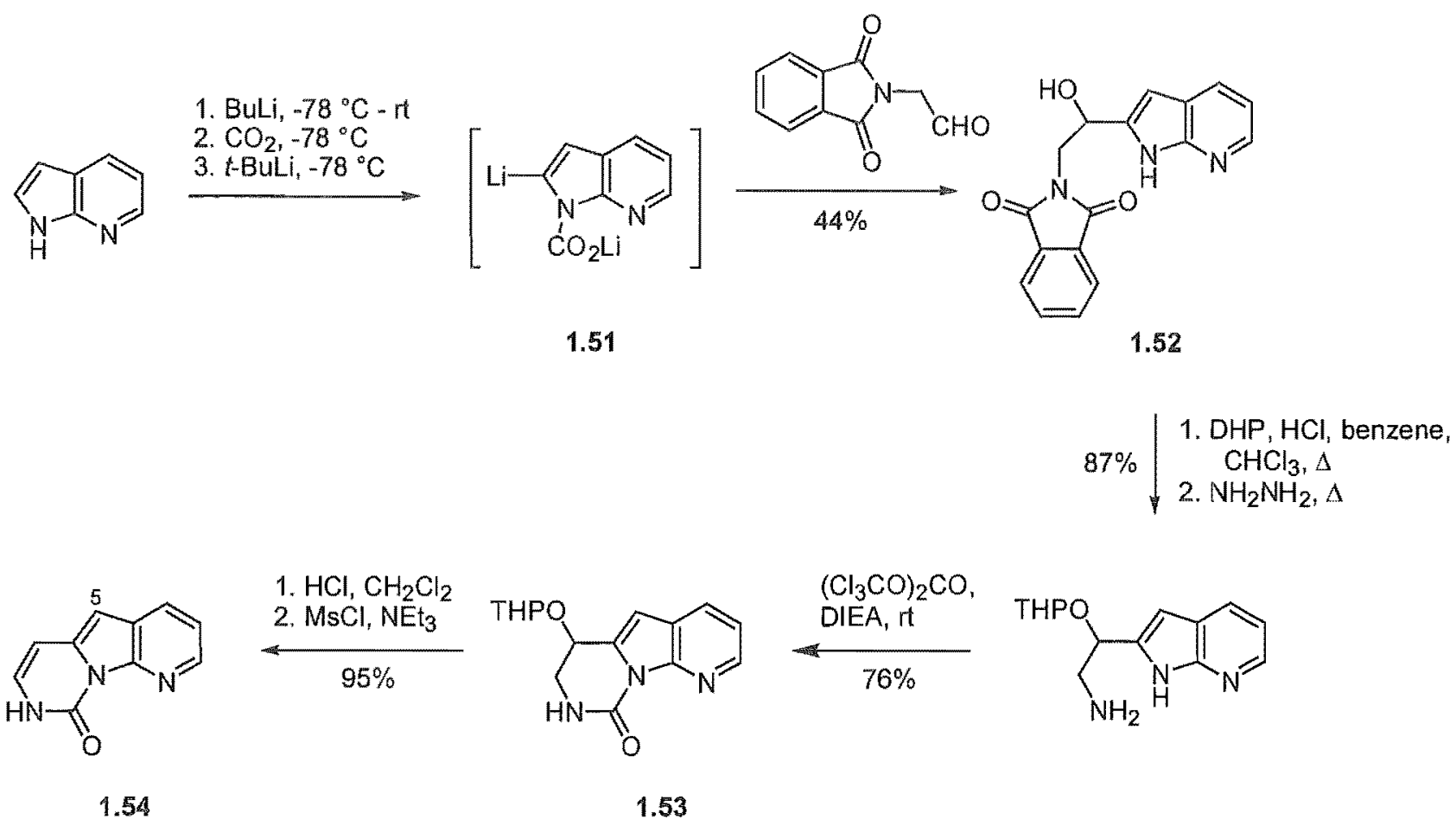

Scheme 1.6

Their strategy for introduction of the 2-aminopyrimidine ring was based on their earlier work, which had been conducted on a simpler system. ${ }^{35 b}$ In this paper, stannane 1.55, which was obtained from the corresponding bromide 1.56 , was coupled with a range of aryl and heteroaryl halides under Pd-catalysis, to give aryl-susbstituted azaindoles 1.57 (Scheme 1.7).<smiles>[R]n1cc(Br)c2cccnc21</smiles>

1.56

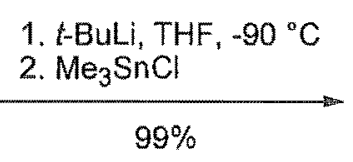

$99 \%$<smiles>[R]n1cc([AsH2])c2cccnc21</smiles>

1.55
1. $\mathrm{Pd}\left(\mathrm{PPh}_{3}\right)_{4}, \mathrm{ArX}$

$\mathrm{LiCl}, \mathrm{THF}$, reflux

2. $10 \%$ aq $\mathrm{HCl}, \mathrm{Ht}$<smiles>Brc1c[nH]c2ncccc12</smiles>

1.57

\begin{tabular}{lcc}
\hline Ar & $X$ & $\%$ Yield \\
\hline 4-methoxyphenyl & $\mathrm{Br}$ & 38 \\
4-nitrophenyl & $\mathrm{Br}$ & 50 \\
thien-3-yl & $\mathrm{Br}$ & 57 \\
2-(methylthio)pyrimidin-4-yl & $\mathrm{Cl}$ & 60 \\
2-(methylthio)pyrimidin-5-yl & $\mathrm{Br}$ & 53 \\
\hline
\end{tabular}


However, application of this methodology to the variolin system presented some difficulties which had to be overcome. While 1.54 could be efficiently halogenated at the 5-position (Scheme 1.8), the halo-derivatives 1.58 could not be efficiently converted to the corresponding 5-stannyl compounds. Neither could 1.58 (Hal $=\mathrm{I}, \mathrm{R}=$ MOM) be coupled with the stannylpyrimidine 1.59. To circumvent these difficulties, it was decided to convert 1.54 to the amine 1.60. This was achieved in a two-step procedure involving $O$-silylation followed by nucleophilic substitution with ammonia in a pressure vessel. After $N$-acylation, selective iodination at $\mathrm{C} 5$ gave 1.61, which participated in $\mathrm{Pd}$-catalysed coupling with 1.59 more readily than did the corresponding 5-halopyrimidinones 1.58 . Finally, $S$-oxidation of 1.62 , followed by substitution of the resulting sulfone with ammonia, produced the target compound deoxyvariolin B (1.50).

TMSCI, HMDSA,

2,6-lutidine

2. $\mathrm{NH}_{3}, 150^{\circ} \mathrm{C}, 60 \mathrm{psi}$

1.54<smiles>Nc1nccc2cc3cccnc3n12</smiles>

1. $A c_{2} \mathrm{O}$, $\mathrm{rt}$

2. $\mathrm{NIS}, \mathrm{CHCl}_{3}, \mathrm{H}$

$71 \%$<smiles>CC(C)Nc1nccc2c(I)c3cccnc3n12</smiles>

1. $N$-protection

2. NBS or $\mathrm{I}_{2}$ or NIS

1.60

1.61<smiles></smiles>

1.58<smiles>Nc1nccc(-c2c3cccnc3n3c(N)nccc23)n1</smiles>

1.50
1. $m$-CPBA, it

2. aq $\mathrm{NH}_{4} \mathrm{OH}$, dioxane, $80^{\circ} \mathrm{C}$

$81 \%$

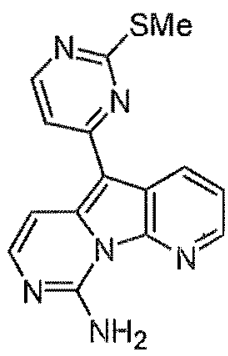

1.62

deoxyvariolin $\mathrm{B}$ 
A notable feature of this synthesis is the selective iodination at C5 of the variolin core, and subsequent introduction of the intact heterocycle. This methodology should allow introduction of a wide range of heterocycles (and other groups) at $\mathrm{C5}$, providing access to a variety of useful analogues. The synthesis is, however, quite a lengthy sequence for a compound of relative structural simplicity, and this situation is likely to worsen if the strategy was applied to variolin $\mathrm{B}$ (1.17). The overall yield for the 14 -step sequence was $2.2 \%$.

\subsubsection{Work Described in this Thesis}

As demonstrated in all the aformentioned syntheses of the variolin core, disconnection of the C9-N10 bond (Figure 1.3, p 18), or double-disconnection of the N8-C9 and C9-N10 bonds, gives rise to a 2-substituted azaindole, which in turn, must be built up from simpler building blocks. Once the variolin core is constructed, further steps must be taken to introduce the heterocycle at $\mathrm{C} 5$. Examination of variolin B revealed another possible disconnection, which could lead to a much simplified, more direct synthesis compared to those mentioned above.

The basis of the new approach was to make the key disconnection between $\mathrm{N} 10$ and $\mathrm{ClOa}$ (Figure 1.4). This would give rise to a synthon in which a negative charge rests on the pyrimidine nitrogen, and a positive charge is placed at the electrophilic 2-position of the pyridine ring, thus exploiting the natural reactivity found in both of these units.

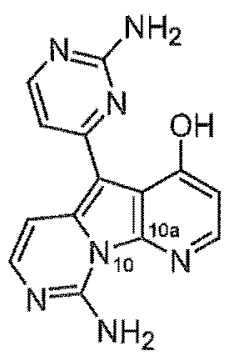

1.17

variolin $\mathrm{B}$<smiles>NC1=NC=CC(=C(c2ccnc(N)n2)c2cnccc2O)[N-]1</smiles><smiles>C1#CCCC#C1</smiles><smiles>C1CCC1</smiles>

Figure 1.4 Disconnection of the N10-C10a bond of variolin B (1.17). 
An appealing feature of this strategy lies in the realisation that a suitable synthetic equivalent for this synthon would be a triarylmethane substituted with a pyridine ring and two identical pyrimidine units. As noted by Corey and Cheng, structural symmetry can usually be exploited to reduce the length or complexity of a synthesis. ${ }^{46}$

In designing the synthesis of variolin B (1.17), it would be prudent to perform some functional group interconversions (FGIs) before disconnection of the $\mathrm{N10}-\mathrm{C} 10 \mathrm{a}$ bond is carried out, so as to avoid having to carry the polar amino groups throughout the synthesis. Thiomethyl groups are often used as synthetic equivalents for amines due to their relative non-polarity and stability, and should be able to be converted to the amines late in the synthesis. ${ }^{47}$ The hydroxyl function would also require protection, most easily as the methyl ether. Numerous methods exist for the deprotection of methyl aryl ethers, ${ }^{48}$ which again, could be conducted after the key bond-forming reactions had been carried out. Implementation of these FGIs reduces the problem to a synthesis of 1.63 (Figure 1.5).

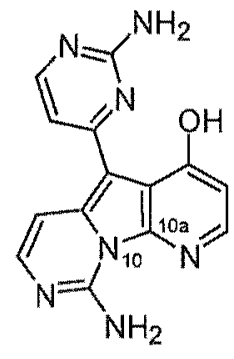

1.17

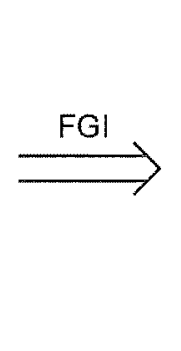

\section{variolin $\mathrm{B}$}

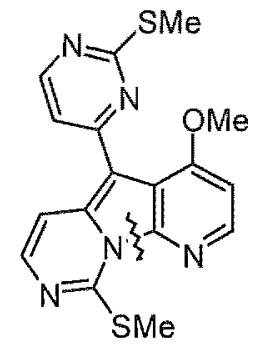

1.63<smiles>[X]c1nccc(OC)c1C(c1ccnc(C)n1)c1ccnc(SC)n1</smiles>

1.64<smiles>C=CC(=[Cr])C=C</smiles><smiles>[X]c1nccc(OC)c1C(O)(c1ccnc(SC)n1)c1ccnc(C(C)C)n1</smiles><smiles>COc1nccc(Cl)n1</smiles>

1.66<smiles>[X]c1nccc(OC)c1C(=O)Cl</smiles>

1.67
1.65

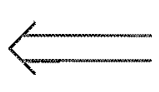

$\mathrm{X}=$ leaving group

Figure 1.5 Retrosynthetic analysis of variolin B (1.17). 
Disconnection of the $\mathrm{N} 10-\mathrm{C} 10 \mathrm{a}$ bond of 1.63 gives the symmetrical triarylmethane 1.64 , where $\mathrm{X}$ would be a suitable leaving group. The triarylmethane could conceivably be produced by reduction of the tertiary benzylic alcohol 1.65 , which itself could result from the reaction of two equivalents of the known ${ }^{49}$ lithiated pyrimidine 1.66 with acid chloride 1.67 .

It is to be noted that this strategy prescribes a highly convergent process, whereby all the carbons and ring-nitrogens present in the target are assembled in a single step. It exploits the natural reactivity of the pyrimidine and pyridine rings, as well as the symmetry embedded in the structure of variolin $B$. The method would not be so well-suited to the other naturally occurring variolins, as it specifically relies on the symmetry found in variolin B (1.17). Having said this, it is possible that the other variolins may be accessible by further functionalisation of variolin $B$ itself. In addition to this possibility, there is the probability that a range of analogues should be accessible by substitution of the thiomethyl groups with various amines or other nucleophiles.

The substituent X would have to be carefully selected-it needs to be a good leaving group, yet it must be stable to a range of reaction conditions, which may include low-temperature lithiations and reducing conditions. It was felt that the chloro group would be an appropriate choice.

While acid chloride $1.67(\mathrm{X}=\mathrm{Cl})$ has not been reported in the literature, 2-chloronicotinoyl chloride (in which the methoxy group of $1.67(\mathrm{X}=\mathrm{Cl}$ ) is replaced by a proton) is available from commercial sources. ${ }^{50}$ It was decided, therefore, to initially investigate the potential of the strategy using 2-chloronicotinoyl chloride as a convenient starting material. The main purpose was to determine the feasibility of this approach to the variolin core. It could also result in a synthesis of deoxyvariolin B (1.50), which would serve as a useful analogue for biological studies. If the methodology proved successful, application to the acid chloride $\mathbf{1 . 6 7}$ would provide a potential method for the synthesis of variolin B (1.17). 


\section{CHAPTER Two}

\section{Synthesis of the Variolin Core}

and

\section{Deoxyvariolin $B$}




\subsection{Introduction}

As discussed in Chapter One, it was decided to first investigate the synthesis of the pyrido[3',2:4,5]pyrrolo[1,2-c]pyrimidine ring system found in all the variolins. As convenient starting materials were available for the deoxy-system, efforts were initially focused on the synthesis of the deoxyvariolin core structure 2.1. If successful, this could be extended to a synthesis of deoxyvariolin B (1.50).

The retrosynthetic strategy for deoxyvariolin B is outlined in Figure 2.1, with the key disconnection being between N10 and C10a of the variolin core 2.1. It was anticipated that this bond could be formed by an addition-elimination reaction of triarylmethane 2.2 . To generate this system, it was decided to investigate the deoxygenation of triarylmethanol $\mathbf{2 . 3}$, as it was felt that this could be readily generated from commercially available 2-chloronicotinoyl chloride (2.4) and lithio-species 1.66.

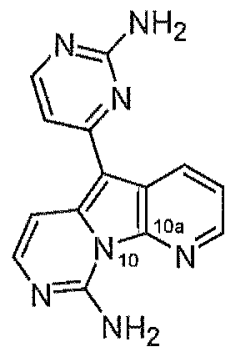

1.50
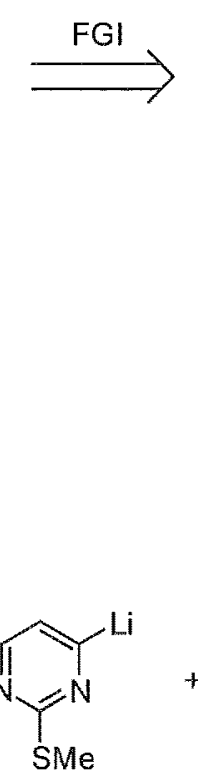

1.66<smiles>CSc1nccc(-c2ccnc(SC)c2-c2ccnc(SC)n2)n1</smiles>

2.1

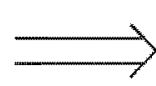<smiles>CSc1nccc(C(c2ccnc(SC)n2)c2cccnc2Cl)n1</smiles>

2.2

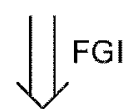<smiles>CSc1nccc(C(O)(c2ccnc(SC)n2)c2cccnc2Cl)n1</smiles>

2.3

Figure 2.1 Retrosynthetic analysis of deoxyvariolin B (1.50). 


\subsection{Synthesis of Triarylmethanol 2.3}

Non-heteroaromatic triarylmethanols have enjoyed much attention in the literature as precursors to stabilised triarylmethyl carbocations. ${ }^{51}$ Generally, triarylmethanols are synthesised using either aryllithium or aryl-Grignard reagents with suitable electrophiles. For $C_{3}$ symmetrical triarylmethanols, reaction of three equivalents of an aryllithium species with diethyl carbonate is a typical procedure (Figure 2.2a). ${ }^{51 f}$

a

b

$$
\operatorname{Ar}-M+
$$<smiles></smiles>

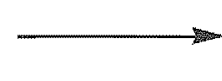

c

$$
2
$$$$
\operatorname{Ar}-\mathrm{M}+
$$<smiles>[R]OC(=O)[Al]</smiles><smiles></smiles>

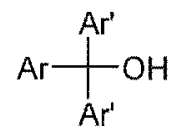<smiles>OC([Al])([Al])[Al]</smiles><smiles>OC([Al])([Al])[Al]</smiles>

$$
M=L i \text { or } M g X
$$

Figure 2.2 Variations on the use of polar organometallics to form triarylmethanols.

Even very hindered products have been produced from such reactions, although high temperatures and extended reaction times are often required. For example, the hexa-o-methoxysubstituted product $\mathbf{2 . 5}$ was formed in $80 \%$ yield upon reaction of the aryllithium species with diphenyl carbonate at $80^{\circ} \mathrm{C}$ for $12 \mathrm{~h}^{51 \mathrm{~b}}$ Less hindered products have been produced under milder conditions. For example, tetra- $o$-methoxy-substituted products, as represented by $\mathbf{2} .6$, were formed in $78 \%$ yield, upon reaction of the appropriate aryllithium species with a symmetrical biaryl ketone in diethyl ether at room temperature for $7 \mathrm{~h}$ (Figure 2.2b). ${ }^{51 \mathrm{c}}$ Alternatively, aryl-Grignard reagents (two equivalents) have been added to arylcarboxylic esters to produce triarylmethanols in 71-95\% yields (Figure $2.2 \mathrm{c}$ ). ${ }^{52}$ 


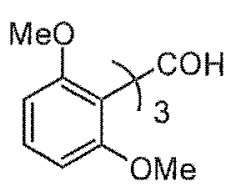

2.5

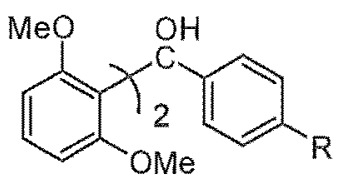

2.6

Application of such methodology to the synthesis of 2.3 requires lithiation of a $\pi$-deficient heterocycle - a widely used tool, but one which deserves some comment. Much work has been done with heteroaryllithium and heteroaryl-Grignard reagents, which have been formed by direct metallation (usually with the aid of an ortho-directing group, although this is not always required $)^{53}$ or by halogen-metal exchange of a suitably functionalised heterocycle. ${ }^{49,54,55,56}$

With $\pi$-deficient heterocycles, low temperatures (usually $-78^{\circ} \mathrm{C}$ or below) are required for optimum results due to the electrophilic nature of the azomethine bond (N1-C2 in pyridine and N1-C6 in pyrimidine), which is readily attacked by nucleophiles at higher temperatures. The pyrimidine azomethine linkage (N1-C6) is more susceptible to nucleophilic attack than that of pyridine, ${ }^{57}$ and pyrimidines have been studied correspondingly less than pyridines. In orthodirected metallations of $\pi$-deficient aromatics, highly hindered bases such as lithium diisopropylamide (LDA) and lithium 2,2,6,6-tetramethylpiperidide (LTMP) have found widespread use for deprotonation, as they have a much lesser propensity to act as nucleophiles than alkyllithiums. ${ }^{53}$ The heteroaryllithiums that are generated, however, are very reactive species which may undergo dimerisation or polymerisation before the electrophile can be introduced. $^{55,58}$ When halogen-metal exchange is used to form the heteroaryllithium, the more nucleophilic alkyllithium reagents must be used.

An example which typifies these difficulties is given below. Gronowitz and Röe have found that 5 -lithiopyrimidine could be formed by halogen-metal exchange of 5 -bromopyrimidine with $n$ BuLi at $-105^{\circ} \mathrm{C}^{55}$ Quenching with $\mathrm{CO}_{2}$ produced the 5-substituted carboxylic acid 2.7 in $68 \%$ yield (Scheme 2.1). If the lithiation is carried out at $-80^{\circ} \mathrm{C}$, however, only a trace of 2.7 was obtained. The lithio-species does form, but it immediately reacts with starting material, adding to the 3,4-azomethine linkage to produce the 3,4-dihydro product 2.8 , which was isolated from the aqueous phase. Small amounts of 2.9 were also produced, which presumably arises as a result of aerial oxidation of 2.8 . When the reaction was conducted at temperatures above $-30{ }^{\circ} \mathrm{C}$, 
direct addition of $n$-BuLi predominated over halogen-metal exchange, giving 2.10 as the major product.<smiles>Brc1cncnc1</smiles>

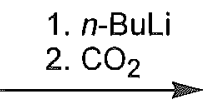<smiles>O=C(O)c1cncnc1</smiles>

2.7<smiles>BrC1=CN=CNC1c1cncnc1</smiles>

2.8<smiles>Brc1cncnc1-c1cncnc1</smiles>

2.9<smiles>BrC1=CN=CNC1Br</smiles>

2.10

\section{Scheme 2.1}

However, if care is taken with the selection of reagents and reaction conditions (altering base, solvent, additives and reaction temperature), it is possible to successfully metallate a wide variety of $\pi$-deficient aromatics, quenching with all manner of electrophiles. In some cases, selectivity can often be achieved for different positions on the ring, ${ }^{59}$ constituting a powerful tool in the functionalisation of heterocycles.

The use of 4-lithio-2-(methylsulfanyl)pyrimidine (1.66) appealed for a direct synthesis of triarylmethanol 2.3. Reaction of two equivalents of 1.66 with 2-chloronicotinoyl chloride (2.4) should give 2.3 through the intermediacy of ketone 2.11 (see Figure 2.3).<smiles>CSc1nccc(Cl)n1</smiles>

1.66<smiles>O=C(Cl)c1cccnc1Cl</smiles>

2.4<smiles>CSc1nccc(C(=O)c2cccnc2Cl)n1</smiles>

2.11<smiles>CSc1nccc(C(O)(c2ccnc(SC)n2)c2cccnc2Cl)n1</smiles>

2.3

Figure 2.3 Proposed reaction of 4-lithio-2-(methylsulfanyl)pyrimidine (1.66) with acid chloride 2.4 to form 2.3 . 
Lithio-species 1.66 was first reported by Undheim and Sandosham, ${ }^{49}$ being formed from the corresponding iodide $\mathbf{2 . 1 2}$ by halogen-metal exchange with $n$-BuLi (Scheme 2.2). The iodide is available in one step from commercially available 4-chloro-2-(methylsulfanyl)pyrimidine (2.13). ${ }^{60}$ Presumably 2.13 is not reactive enough to undergo halogen-metal exchange with $n$ BuLi. The lithiation conditions involve stirring iodide 2.12 and $n-\mathrm{BuLi}$ at $-95{ }^{\circ} \mathrm{C}$ for $1 \mathrm{~h}$, followed by addition of the electrophile (in the original paper, tributylstannyl chloride) and gradual warming to room temperature. Stannane 2.14 was produced in $76 \%$ yield. Interestingly, the stannane was coupled with heterocycles 2.15 and 2.16 to afford the biaryl products 2.17 and 2.18 in good yields. It is remarkable that reaction of 2.14 with 2.16 proceeds at $-78{ }^{\circ} \mathrm{C}$ and requires no metal catalysis.

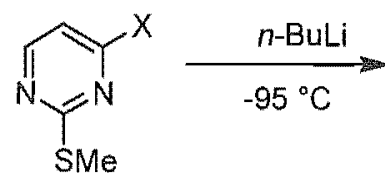

$\underset{76 \%}{\operatorname{aq~} H I, \mathrm{rt}}\left[\begin{array}{l}2.13 X=\mathrm{Cl} \\ 2.12 X=1\end{array}\right.$

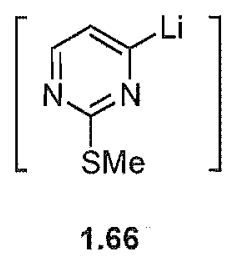

2.14

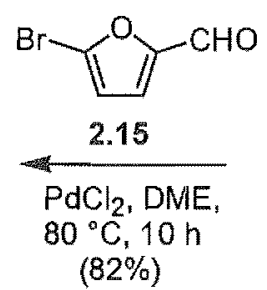

2.17<smiles>CSc1nccc(-c2ccc(C=O)o2)n1</smiles>

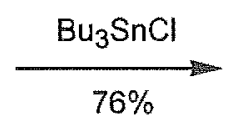<smiles>CCCCCCCCCCCCC</smiles>

2.14

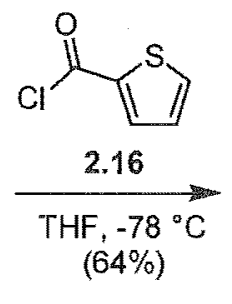

$(64 \%)$<smiles>CSc1nccc(C(=O)c2cccs2)n1</smiles>

2.18

Scheme 2.2

Thus, the synthesis of triarylmethanol 2.3 began with preparation of the iodide 2.12 from 2.13 according to Undheim's procedure ${ }^{60}$ (Scheme 2.3). This involved stirring the chloride 2.13 in $55 \%$ aqueous $\mathrm{HI}$ solution for $48 \mathrm{~h}$ at room temperature. Efficient stirring of the thick sludge thus produced was necessary to obtain the maximum yield, and it was important that any lumps were regularly broken up with a glass rod to give a homogeneous brown paste. After workup with aqueous $\mathrm{NaHCO}_{3}$ solution, recrystallisation from hexanes gave iodide 2.12 as large white needles in $79 \%$ yield. 
The other component required for the synthesis of 2.3 is 2-chloronicotinoyl chloride (2.4). While this compound is commercially available, it was found that hydrolysis to the carboxylic acid occurs slowly, even when carefully stored under anhydrous conditions. It was more convenient (and economical), therefore, to freshly prepare it from 2-chloronicotinic acid (2.19) one day before use. This was effected in $97 \%$ yield by refluxing in thionyl chloride ${ }^{61}$ with catalytic DMF, and distilling the crude material under vacuum in a Kugelrohr oven.

Using the lithiation protocol reported by Undheim and Sandosham, ${ }^{49}$ a solution of iodide 2.12 in $\mathrm{THF}$ at $-95{ }^{\circ} \mathrm{C}$ was treated with $n$-BuLi. After $1 \mathrm{~h}$ at $-95{ }^{\circ} \mathrm{C}, 0.5$ equivalents of 2 chloronicotinoyl chloride (2.4) in THF were added and, after $15 \mathrm{~min}$, the temperature was allowed to slowly rise to room temperature. The ${ }^{1} \mathrm{H}$ NMR spectrum of the crude reaction mixture indicated the presence of at least six products. Flash chromatography allowed the isolation of the major product, which was determined to be the desired triarylmethanol $\mathbf{2 . 3}$.

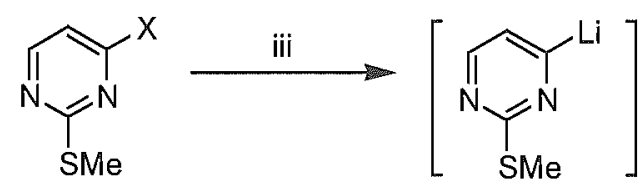

1.66<smiles>O=C(Cl)c1cccnc1Cl</smiles><smiles>C#CC#N</smiles>

2.4<smiles>O=C(O)c1cccnc1Cl</smiles>

2.19

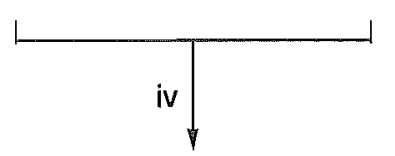<smiles>CSc1nccc(C(O)(c2ccnc(SC)n2)c2cccnc2Cl)n1</smiles>

2.3

Scheme 2.3 Reagents and yields: (i) $55 \%$ aq $\mathrm{HI} / \mathrm{rt} \mathrm{(79 \% );} \mathrm{(ii)} \mathrm{SOCl}_{2} / \mathrm{cat}$. DMF/reflux (97\%); (iii) $n$-BuLi (1 equiv)/THF/-95 ${ }^{\circ} \mathrm{C}, 1$ h; (iv) 2.4 ( 0.5 equiv)/THF/-95 ${ }^{\circ} \mathrm{C}$ to $\mathrm{rt}(20 \%)$. 
The ${ }^{1} \mathrm{H}$ NMR spectrum of 2.3 is shown in Figure 2.4. The 2,3-substituted pyridine unit gives rise to a distinctive $\mathrm{ABX}$ splitting pattern, while the identical pyrimidine rings produce a pair of doublets, each of which integrates for two protons. A sharp singlet is seen for the hydroxyl proton at $\delta 6.33$, and a large singlet at $\delta 2.49$ represents the six thiomethyl protons. The mass spectrum displays a molecular ion at $\mathrm{m} / \mathrm{z}$ 391, as expected for 2.3. Interestingly, the base peak in this spectrum has a mass of 356 , which corresponds to a loss of chlorine. While simple 2chloropyridines can fragment in this manner, ${ }^{62}$ the loss of chlorine from 2.3 appears to be a much more facile process. It is thought that the alcohol may be cyclising under the MS conditions to give a pyridopyrrolopyrimidine-type species. It was hoped that this would be indicative of things to come.

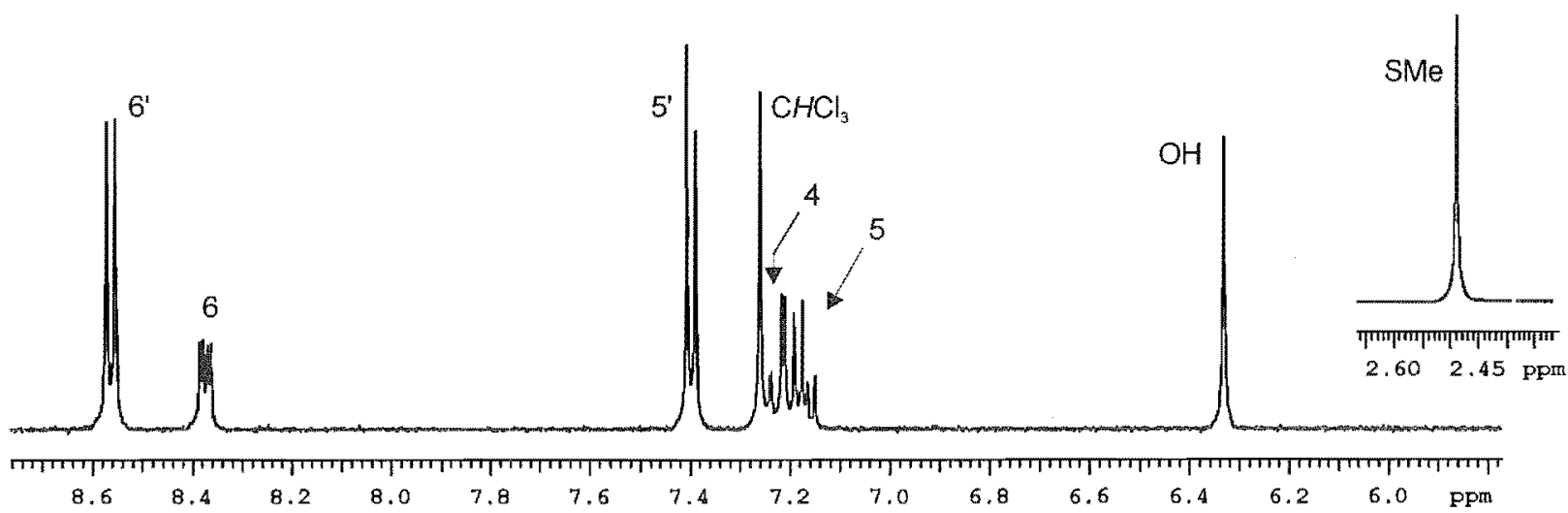

Figure 2.4 $300 \mathrm{MHz}{ }^{1} \mathrm{H}$ NMR spectrum of triarylmethanol 2.3.

Unfortunately, however, the isolated yield of 2.3 was only $\sim 20 \%$. Other products identified in the reaction fell into two classes - those which were simply pyrimidine-containing byproducts of the lithiation step (2.20-2.22), and those containing both a pyridine and a pyrimidine ring (2.11 and 2.23). The 2,3-disubstituted pyridine moiety was easily recognised by its distinctive longrange coupling in the ${ }^{1} \mathrm{H}$ NMR spectrum, whereas the 2,4-disubstituted pyrimidines give rise to simple AX splitting patterns. 
<smiles>CSc1ncccn1</smiles>

2.20<smiles>CSc1nccc(-c2ccnc(SC)n2)n1</smiles>

2.21<smiles>CSc1nccc(-c2cc(-c3ccnc(SC)n3)nc(SC)n2)n1</smiles>

2.22<smiles>CSc1nccc(C(=O)c2cccnc2Cl)n1</smiles>

2.11<smiles>CSc1nccc(C(O)c2cccnc2Cl)n1</smiles>

2.23

The major byproduct was the deiodinated pyrimidine 2.20 , being present in almost the same proportion as $\mathbf{2 . 3}$ (judging from the ${ }^{1} \mathrm{H}$ NMR spectrum of the crude material). It must be formed by the quenching of the lithio-species 1.66 with an adventitious proton source. Pyrimidine 2.20 could not arise from protonation of $\mathbf{1 . 6 6}$ upon workup, as $\mathbf{1 . 6 6}$ would have decomposed long before the reaction had reached room temperature. Significant quantities of $\mathbf{2 . 2 0}$ were always obtained, despite using standard inert atmosphere techniques, base-washed glassware and freshly purified solvents and reagents. It is possible that 2.20 may result from deprotonation of the solvent, THF. Such reactions have been documented with $n$-BuLi at room temperature, which quantitatively produces the enolate of acetaldehyde after $16 \mathrm{~h}$ (see Figure 2.5 ) ${ }^{63}$ It is surprising that the aryllithium $\mathbf{1 . 6 6}$ (which is presumed to be less basic than $n-\mathrm{BuLi}$ ) is reactive enough to deprotonate THF at temperatures well below room temperature.
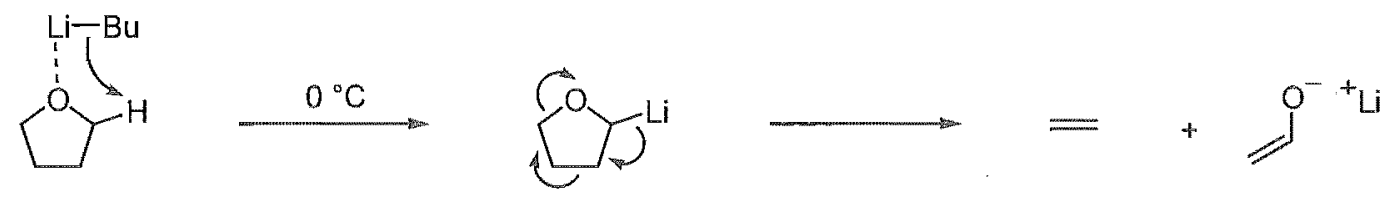

Figure 2.5 Deprotonation of THF by $n$-BuLi.

The presence of products $2.20-2.22$ indicate that the lithio-species 1.66 is unstable and can react via a number of pathways which compete with the addition to the acid chloride. Presumably 
tributylstannyl chloride, the electrophile used by Undheim and Sandosham, is reactive enough that these competing pathways do not present a significant problem. ${ }^{49}$ The bipyrimidine 2.21 is formally the result of a Wurtz coupling ${ }^{64}$ (Figure 2.6a), although there are several alternative processes that could be considered. For example, addition of $\mathbf{1 . 6 6}$ to $\mathbf{2 . 1 2}$, followed by elimination of LiI would give rise to the bipyrimidine (Figure 2.6b). However, $\pi$-deficient heteroaromatics can also undergo addition to unsubstituted azomethine bonds, as in Figure 2.6c, in preference to those with a substituent on the carbon. ${ }^{65}$ The resulting anion 2.24 could undergo halogen-metal exchange to form the dianion 2.25 which, when protonated and oxidised in air, would produce $\mathbf{2 . 2 1}$.<smiles>COc1nccc(I)n1</smiles>

1.66 a

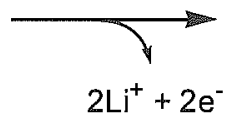

$2 \mathrm{Li}^{+}+2 \mathrm{e}$

b

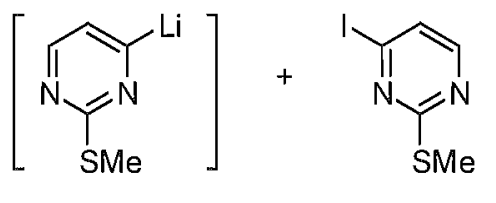

1.66<smiles>CSc1nccc(-c2ccnc(SC)n2)n1</smiles>

2.21<smiles>CSC1=NC=CC(I)(c2ccnc(SC)n2)N1[In+]</smiles>

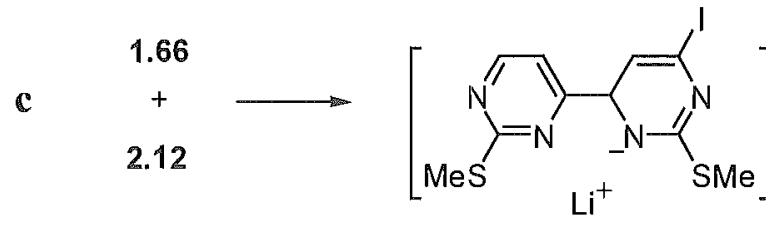

2.24

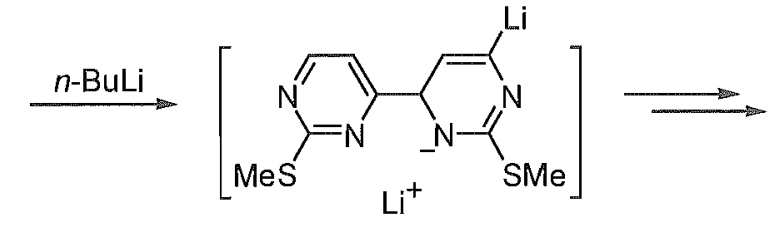

2.25

Figure 2.6 Possible mechanisms of formation of the bipyrimidine product 2.21 .

The presence of $\mathbf{2 . 2 2}$ can be explained by a combination of process (c) and either processes (a), (b) or (c). For example, (c) + (a) would involve formation of dianion 2.25 which reacts with another equivalent of lithio-species 1.66 to produce 2.26 . Oxidative workup of 2.26 would result in the formation of 2.22 (Figure 2.7). 


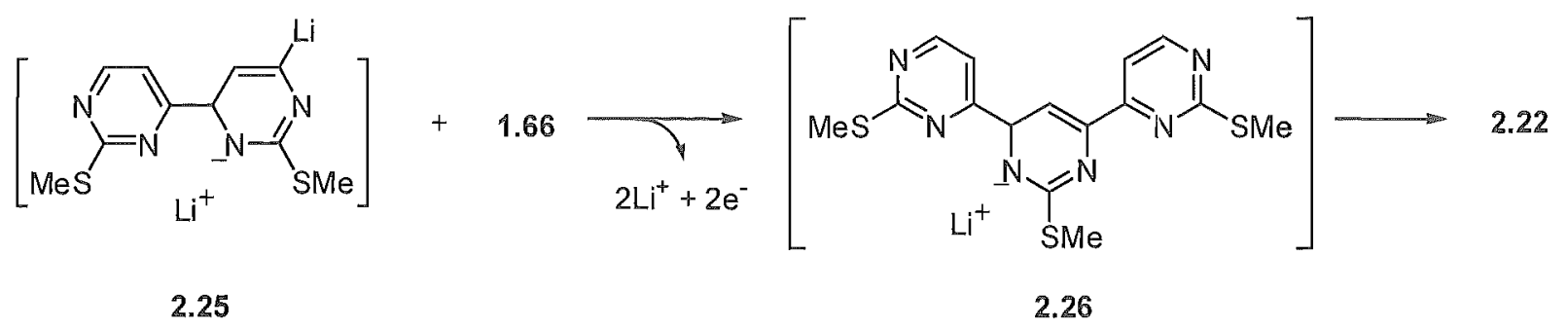

Figure 2.7 Possible mechanism of formation of 2.22.

The ketone product 2.11 is an expected intermediate en route to triarylmethanol 2.3. Its presence indicates that not enough $\mathbf{1 . 6 6}$ was available for complete reaction-a hardly surprising result based on the presence of products $2.20-2.22$. The biarylmethanol 2.23 , however, was unexpected and is not so easily explained. Common sense would suggest that it is formed from the ketone $\mathbf{2 . 1 1}$ by an adventitious reducing agent. For example, if route (a) (Figure 2.6a) is involved in the production of $\mathbf{2 . 2 1}$, the electrons produced would be expected to readily reduce the benzylic ketone $\mathbf{2 . 1 1}$, thereby explaining the formation of $\mathbf{2 . 2 3}$.

The conclusion from all this conjecture is that the lithio-species $\mathbb{1 . 6 6}$ is an extremely reactive intermediate which requires careful control of the reaction conditions to prevent it reacting with itself or unreacted iodide 2.12. It was surmised that side-products 2.20-2.22 most likely form upon warming of the reaction to room temperature. These products were expected to be minimised, therefore, if the following stipulations were adhered to: slow addition of precooled reagents (both $n-\mathrm{BuLi}$ and acid chloride 2.4) to avoid an exotherm in the reaction mixture; maintenance of low temperature until the reaction is complete; and dilution of the reaction mixture (this should disfavour dimerisation of 1.66 to give 2.21 ).

To this end, a specialised piece of glassware was used to conduct the reaction in, shown in Figure 2.8. The reactor was first described by Suzuki and Noyori, who required careful control of temperature in their prostaglandin synthesis. ${ }^{66}$ The reactor is immersed in the cooling bath up to the level of the top coil. Addition of reagents through the coiled side-arm ensures that these solutions are already cooled to the temperature of the external bath before making contact with the bulk reaction. 


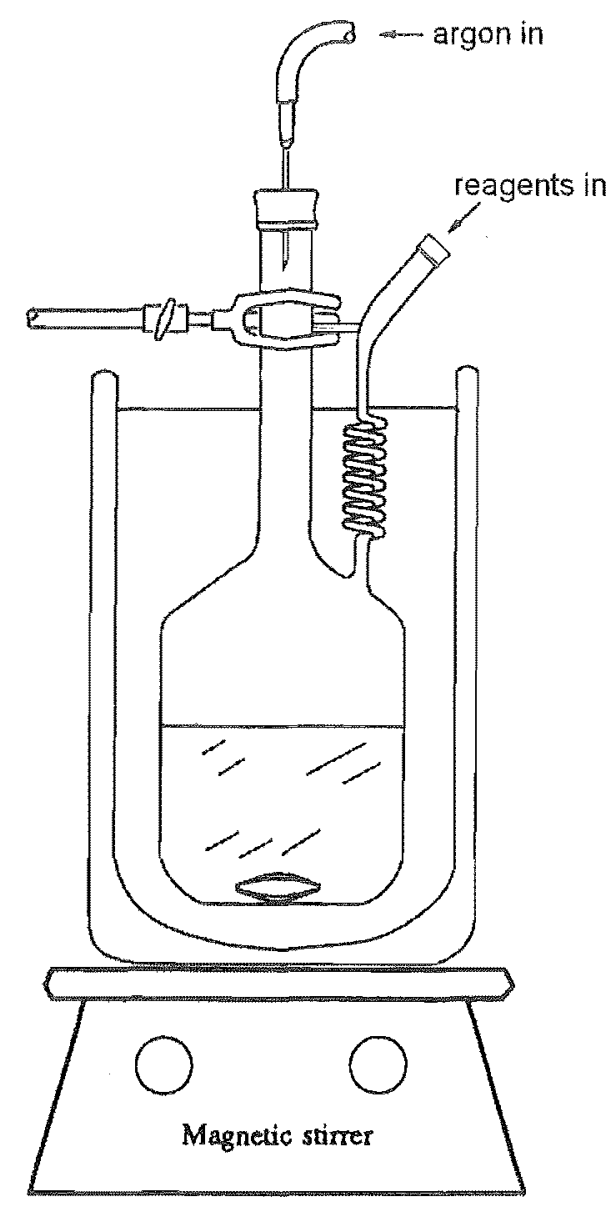

Figure 2.8 Reactor used to carry out large-scale lithiations of $\mathbf{2 . 1 2}$.

Use of the reactor in the lithiation of iodide 2.12 , with application of the aforementioned stipulations, led to significantly cleaner reactions. The reactor was particularly useful for large scale reactions, in which internal exotherms were a significant problem. The side-products 2.11 and 2.20-2.23 were still observed in the reaction, but in much lower proportions.

It was important to ascertain exactly how long was required for complete reaction to occur at -95 ${ }^{\circ} \mathrm{C}$. This was determined by using methanol to quench the reaction (while still at $-95^{\circ} \mathrm{C}$ ) after a fixed period. If any unreacted acid chloride 2.4 still remained, it would be identified in the ${ }^{1} \mathrm{H}$ NMR spectrum as the methyl ester. (Quenching with aqueous acid would convert any remaining 2.4 to 2-chloronicotinic acid. This would be extracted into the aqueous phase upon workup and consequently, would not be observed in the ${ }^{1} \mathrm{H}$ NMR spectrum.) If any 2.4 did remain, this could be due to either the reaction not being left for long enough at $-95^{\circ} \mathrm{C}$, and/or insufficient 
lithio-species 1.66 (which presumably would have been consumed via the competing pathways discussed previously). To distinguish between these two possibilities, the reaction was quenched at $-95{ }^{\circ} \mathrm{C}$ with deuterated methanol $\left(\mathrm{CH}_{3} \mathrm{OD}\right)$. If any active lithio-species remained, the deuterated pyrimidine 2.27 would be formed (and any unreacted 2.4 would still be isolated as the methyl ester). Pyrimidine 2.27 can be distinguished from 2.20 in the ${ }^{1} \mathrm{H}$ NMR spectrum, as illustrated in Figure 2.9. Comparison of the ${ }^{1} \mathrm{H}$ NMR spectra for 2.27 and 2.20 (present in the crude mixture of the lithiation reaction) reveals that the chemical shifts do not differ between the two compounds, merely the splitting pattern. The coupling between deuterium and hydrogen is too small to be observed and so 2.27 gives rise to a simple AX pair of doublets (Figure 2.9a). H5 of 2.20, however, couples to its two neighbouring protons and consequently gives rise to a triplet at $\delta 6.95$ (Figure 2.9b). Figure 2.9c shows the ${ }^{1} \mathrm{H}$ NMR spectrum of a reaction mixture quenched at $-95^{\circ} \mathrm{C}$. Even though the signals for 2.20 and 2.27 overlap, it can be clearly seen that 1.66 was still present in the reaction at the time of quenching.

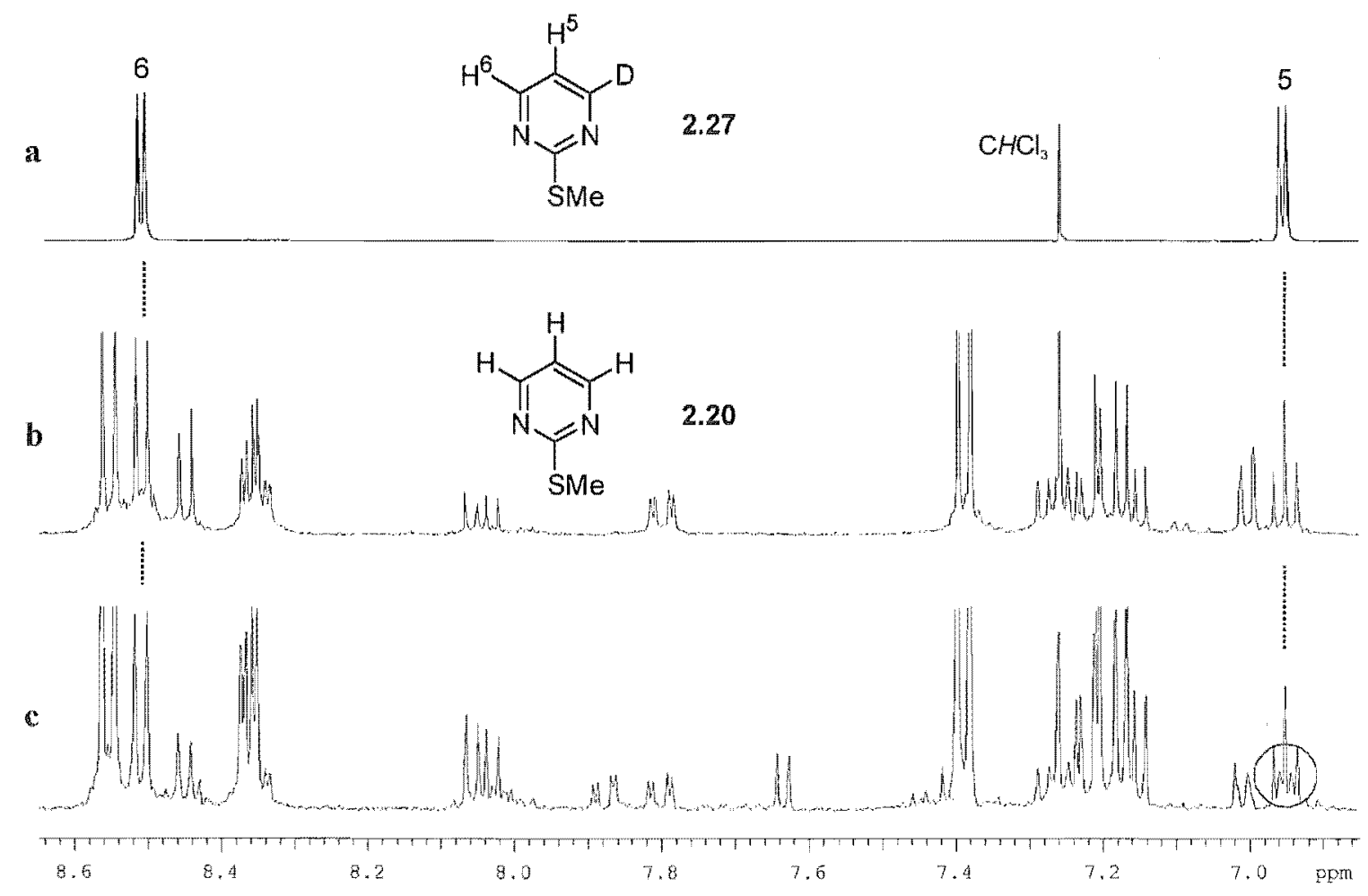

Figure 2.9 Aromatic regions of the ${ }^{1} \mathrm{H}$ NMR spectra of: (a) 4-deuterio-2-(methylsulfanyl)pyrimidine (2.27); (b) lithiation reaction containing 2-(methylsulfanyl)pyrimidine (2.20) as a byproduct; (c) lithiation reaction quenched at $-95^{\circ} \mathrm{C}$ with $\mathrm{CH}_{3} \mathrm{OD}, 2.27$ (circled) was observed, thereby indicating the presence of the lithio-species 1.66 at the time of quenching. 
Thus, by quenching the reaction with $\mathrm{CH}_{3} \mathrm{OD}$ at $-95{ }^{\circ} \mathrm{C}$, it can be determined whether 2.4 or 1.66 remain. If both are still present, the reaction needs to be left longer at $-95^{\circ} \mathrm{C}$ before quenching. If 2.4 remains, but not 1.66 , the reaction requires the use of greater than two equivalents of 1.66 .

After several reactions, the best conditions found involved using three equivalents of $\mathbf{2 . 1 2}$ (with respect to 2.4), and conducting the reaction at $-95^{\circ} \mathrm{C}$ for at least $3.5 \mathrm{~h}$. Under these conditions, the triarylmethanol 2.3 was isolated in 56\% yield (90-95\% purity). As chromatographic separation of the two alcohols 2.3 and 2.23 proved difficult, the partially purified mixture was subjected to oxidation to $\mathrm{MnO}_{2}$ in refluxing benzene. This converted the secondary alcohol to the ketone 2.11 , which was easily removed from triarylmethanol 2.3 by flash chromatography.

Complete elimination of 2.11 and $2.20-2.23$ could not be achieved, hence the need for greater than two equivalents of 2.12. It should be mentioned that extremely careful control of the bath temperature and addition rates of reagents was required for consistent results. As the scale of the reaction was increased beyond $\sim 20 \mathrm{mmol}$ of iodide 2.12 , it became particularly difficult to preclude the formation of 2.20-2.22. Prolonged periods of BuLi-addition (e.g. $60 \mathrm{~min}$ for $30 \mathrm{~mL}$ of $n$-BuLi in hexanes) meant that 1.66 had a significant opportunity to degrade before the acid chloride 2.4 could be added.

In summary, triarylmethanol 2.3 has been generated on a $4.5 \mathrm{mmol}$ scale in $56 \%$ yield (calculated from 2.19, the limiting reagent), starting with the readily available materials 2 chloronicotinic acid (2.19) and 4-chloro-2-(methylsulfanyl)pyrimidine (2.13). This method assembles all the carbons and ring-nitrogens of deoxyvariolin $B$ in a single step. With a successful route to the production of $\mathbf{2 . 3}$ on a gram-scale, its deoxygenation and subsequent cyclisation to the variolin skeleton 2.1 could now be investigated. 


\subsection{Formation of the Variolin Core}

Perusal of the literature reveals numerous methods that have been devised, or discovered, for the deoxygenation of alcohols. Some of these methods are well-established, being in common use for thirty years or more, ${ }^{67}$ while methods are still being reported at the present time. ${ }^{68}$ Two general methods for the deoxygenation of alcohols exist-radical deoxygenation ${ }^{69}$ and ionic hydrogenolysis. Within these two categories, there exist numerous reagent systems that have been developed; such variations are necessary, as the balance required between reactivity and selectivity varies from one application to another.

\subsubsection{Radical Deoxygenation of Triarylmethanol 2.3}

Radical reduction of triarylmethanol $\mathbf{2 . 3}$ may be a feasible process as the triarylmethyl radical 2.28 is expected to be stabilised by the $\pi$-deficient heterocycles. Provided that it could be formed, 2.28 could abstract a hydrogen atom (e.g. from tributylstannane) to produce the triarylmethane $\mathbf{2 . 2}$ or, alternatively, it may cyclise directly to the variolin core 2.1 (Figure 2.10 ). In either case, a chain process can be envisaged due to the elimination of tributyltin or chlorine radicals, respectively. 
<smiles>COc1nccc(C(O)(c2cccnc2)c2cccnc2Cl)n1</smiles>

2.3

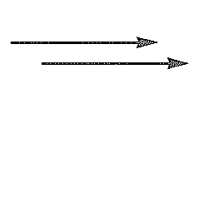<smiles>COc1nccc(C(c2cccnc2)c2cccnc2Cl)n1</smiles>

2.28<smiles>[CH][CH]C(C)C</smiles><smiles>CSc1nccc(-c2c3cccnc3n3c(SC)nccc23)n1</smiles>

2.1

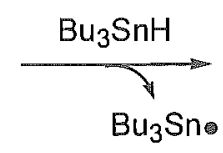<smiles>CSc1nccc(C(c2ccnc(SC)n2)c2cccnc2Cl)n1</smiles>

2.2

Figure 2.10 Proposed use of radical deoxygenation of $\mathbf{2 . 3}$.

\subsubsection{Barton-McCombie Deoxygenation}

Of the numerous procedures described for radical deoxygenation of alcohols, ${ }^{69}$ the most widely used is the Barton-McCombie method, first reported in $1975 .^{70}$ In this procedure, the alcohol is converted into either a thiobenzoate $\mathbf{2 . 3 0}$ or a dithiocarbonate/xanthate 2.31 , which is subsequently reduced with tributyltin hydride in refluxing toluene (Figure 2.11). The reduction proceeds via a chain process, in which addition of a tributylstannyl radical to sulfur gives a carbon-centred radical 2.32, which can then fragment to produce a new radical 2.33. This is intercepted by tributyltin hydride to give the reduced product 2.34 , with regeneration of the tin radical so that the chain process may continue. 
a)<smiles>[R]OC([Na])=S</smiles>

2.31

b)<smiles>[R]OC([R])=S</smiles>

1. 2.29, pyridine 2. $\mathrm{H}_{2} \mathrm{~S}$

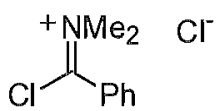

2.29<smiles>[R]OC(=S)c1ccccc1</smiles>

2.30<smiles>[R]OC([R])S[SbH2]</smiles>

2.32<smiles>[R]CSSC([R])=O</smiles>

2.33

2.34

Figure $2.11 \quad$ a) Formation of thiobenzoates and xanthates; b) Tributyltin hydride-mediated reduction of thiobenzoates/xanthates to give alkanes.

Accordingly, functionalisation of triarylmethanol 2.3 was attempted using the standard protocol for xanthate formation (see Figure 2.11a). However, mixtures of products were always obtained, with the major component of the mixture being the starting alcohol 2.3. Despite repeating the reaction with excess reagents and/or longer reaction times, no improvement could be gained. Other groups have also experienced difficulties in preparing (and reducing) hindered xanthates. ${ }^{71,72}$ 


\subsubsection{Deoxygenation of Methyl Oxalyl Esters}

Dolan and MacMillan have described the use of methyl oxalyl esters as alternative radical precursors to xanthates and thiobenzoates. ${ }^{72}$ While preparation of tertiary xanthate and thiobenzoate derivatives of $\mathbf{2 . 3 5}$ proved difficult, the methyl oxalyl esters 2.36 were readily prepared. These products were subsequently reduced by tributyltin hydride, in the presence of a radical initiator, to produce good yields of the 13-deoxygibberellins (Scheme 2.4).

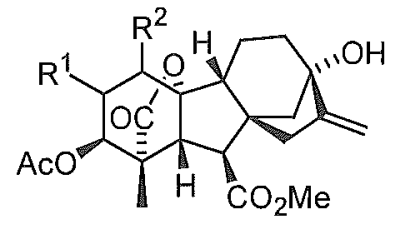

2.35

$$
\begin{aligned}
& R^{1}, R^{2}=H \text { or } \\
& R^{1}, R^{2}=\text { double bond }
\end{aligned}
$$

1. oxalyl chloride, it

2. $\mathrm{MeOH}$

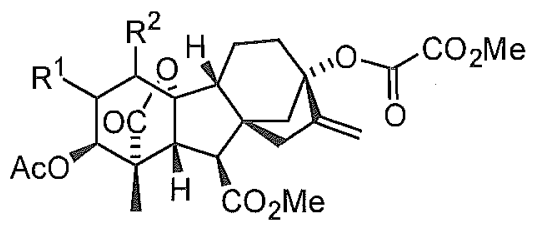

2.36

$\mathrm{Bu}_{3} \mathrm{SnH}$ radical initiator, toluene, reflux

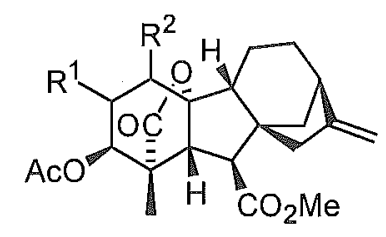

$65 \%$ over two steps for $R^{1}, R^{2}=$ double bond

Scheme 2.4

However when triarylmethanol 2.3 was stirred with oxalyl chloride in dry dichloromethane, followed by methanolic workup, only the starting alcohol was obtained. When triethylamine was included in the reaction, up to three minor products were produced, but the starting alcohol was still the major component of the mixture. It is assumed that the hindered nature of triarylmethanol 2.3 is the cause of its reluctance to undergo functionalisation to either xanthates or methyl oxalyl esters. As a consequence, this line of research was discontinued. 


\subsubsection{Ionic Hydrogenolysis of Triarylmethanol 2.3}

Many methods exist for the direct reduction of alcohols, without the need for functionalisation to a more reactive derivative. As has been mentioned, triarylmethanols have been the subject of a number of studies in regard to their direct deoxygenation to stabilised triarylmethane carbocations. $^{51}$ A benzylic carbocation experiences considerable stabilisation over a normal alkyl carbocation, due to overlap of the empty $p$ orbital of the carbocation with the conjugated $\pi$ system of the aromatic ring. Exceptional stabilisation can be achieved by substituting the carbocation with three aromatic rings. For example, the trityl cation $\mathbf{2 . 3 7}$ is considerably less susceptible to nucleophilic attack than the simple benzyl cation. However, this is largely due to steric effects rather than electronic effects. While it may seem tempting to delocalise the positive charge over all three rings, these resonance structures actually play only a small part in the electronic nature of the molecule. This is because the steric bulk of the aromatic rings forces the cation into a propeller conformation. ${ }^{73}$ This arrangement lessens the extra stabilisation that could be gained from overlap of the $2 p$ orbital of the cation with the aromatic $\pi$-electrons, but increases the stability of the cation by making it more hindered. Further stabilisation of such benzylic cations can be gained if the phenyl rings are substituted with electron-donating groups, such as the methoxy group. This stabilisation is probably a combination of both steric effects (if the substituent is in the ortho position) and electron-donating effects. ${ }^{51 \mathrm{~d}}$ Conversely, electronwithdrawing groups destabilise the cation, as they are unable to assist in delocalisation of the charge.

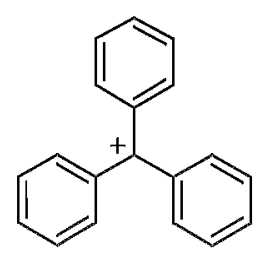

2.37

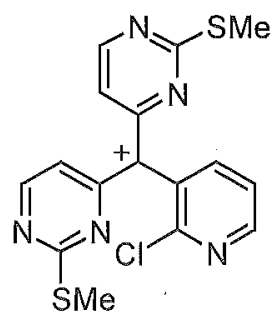

2.38

In the case of triarylmethanol $\mathbf{2 . 3}$, the heteroaromatic groups may play a part in resonancestabilisation of the incipient carbocation $\mathbf{2 . 3 8}$, however this would be offset to some extent, by the highly $\pi$-deficient nature of these rings. It is difficult to predict, in any quantitative manner, 
just how stable the cation may be. Suffice it to say, it was anticipated that more forcing conditions would be required for its formation than are required for the generation of the trityl cation 2.37. If, however, the cation could be produced, it was thought that a suitable hydride source could be found to reduce it to the triarylmethane 2.2 .

Such processes are referred to as ionic hydrogenolyses, and as the name suggests, they proceed by an ionic mechanism and involve an intermediate carbocation. These reactions require a twocomponent reagent system consisting of an acid (Lewis or Brønsted) to ionise the alcohol and a hydride source to reduce the incipient cation. Of special concern in the case of the variolins was the reactivity of heterocyclic systems to such conditions. As quarternised pyridines are known to undergo reduction of the azomethine bond with sodium borohydride or lithium aluminium hydride ${ }^{74}$ the possibility existed that the $\pi$-deficient heterocycles of $\mathbf{2 . 3}$ may not withstand such reducing conditions. Thus, conditions would have to be found of sufficient reactivity to intercept the triarylmethylcation $\mathbf{2 . 3 8}$, yet with the selectivity to leave the $\pi$-deficient heterocycles untouched.

A preliminary screening of the more commonly used methods was carried out to ascertain which, if any, would be most suitable for the deoxygenation of 2.3. These included $\mathrm{NaCNBH}_{3} / \mathrm{ZnI}_{2},{ }^{75}$ Pd-C/AlCl $3 /$ cyclohexene, ${ }^{76} \mathrm{P} / \mathrm{I}_{2} / \mathrm{HI}^{77}{ }^{7} \mathrm{NaBH}_{4} / \mathrm{TFA}^{78} \mathrm{H}_{2} /$ Adams' catalyst $\left(\mathrm{PtO}_{2}\right) / \mathrm{TFA}^{79}$ and $\mathrm{LiAlH}_{4} / \mathrm{AlCl}_{3}{ }^{80}$ In general, these reagent systems returned only starting material or formed complex product mixtures. In some cases, poor mass-return after workup indicated that destruction of the alcohol 2.3 was a problem. Reduction of the aromatic heterocycles may have been responsible, based on the ${ }^{1} \mathrm{H}$ NMR spectra of the crude reaction mixtures.

One reagent system, however, held promise for reduction of triarylmethanol 2.3. This system utilises an organosilane as a mild and selective reducing agent in the presence of an acid (usually trifluoroacetic acid). ${ }^{67}$ This system effectively reduces substituted alkenes, cyclopropanes, certain alcohols, esters and ethers, and aromatic ketones and aldehydes to the corresponding alkanes. A number of other functional groups can also be reduced, including $\alpha, \beta$-unsaturated and saturated ketones, $p$-quinones, disulfides and acetals to give a variety of products. However, the trifluoroacetic acid/organosilane system is tolerant of many functional groups which are reduced by other methods. These include carboxylic acids and simple esters, amides, nitriles, nitro groups, sulfonates and aromatic rings. In addition, many alcohols, ethers, alkenes and alkyl 
halides survive the reaction conditions. Significantly, pyridine, isoquinoline and acridine are all reported to resist reduction by the trifluoroacetic acid/organosilane hydrogenolysis pair. ${ }^{67}$ However, no mention was made regarding pyrimidines. In contrast to these $\pi$-deficient heterocycles, substituted indoles and thiophenes are reduced, to dihydroindoles and tetrahydrothiophenes respectively. ${ }^{67}$

The high yields obtained from the trifluoroacetic acid/organosilane hydrogenolysis system, combined with its wide application, particularly in the reduction of benzylic alcohols, and tolerance of many functional groups gave it significant appeal for the reduction of 2.3 .

Initially, the attempted reaction of 2.3 in $\mathrm{CH}_{2} \mathrm{Cl}_{2}$ at room temperature with 1.2 equivalents of triethylsilane (TES) and 2 equivalents of trifluoroacetic acid (TFA) gave only starting material. Even after refluxing for $30 \mathrm{~h}$, only starting material was isolated. However, when the reaction was carried out in refluxing TFA as solvent (i.e. $\mathrm{CH}_{2} \mathrm{Cl}_{2}$ was not present in the reaction), the starting material did react to form a number of products. As the ${ }^{1} \mathrm{H}$ NMR spectrum of the crude reaction mixture was rather complicated, it was difficult to determine whether the desired triarylmethane 2.2 had formed. To shed some light on the reaction, the mixture was subjected to chromatographic separation. Careful flash chromatography was used to separate the products, however HPLC was required to achieve complete purification.

\subsubsection{Products from the Ionic Hydrogenolysis of Triarylmethanol 2.3}

It should be noted that although many products were formed in the TFA/TES reaction, the products isolated were those that were most readily obtained after silica flash chromatography. They also corresponded (in general) to the more predominant compounds observed in the ${ }^{1} \mathrm{H}$ NMR spectrum of the crude mixture. The poor mass-balance obtained from the reaction, combined with the extensive chromatography that was required, meant that yields for most of the compounds were not determined-the initial focus was to identify the reaction products, and to attempt to ascertain what processes had occurred.

Four products $\mathbf{A}, \mathbb{B}, \mathbb{C}$ and $\mathbb{D}$ were isolated from the reaction. The ${ }^{1} \mathrm{H}$ NMR spectrum of triarylmethane 2.2 - the desired product of the reaction-should contain a rather deshielded singlet for the proton attached to the central carbon. One of the new products $(\mathbb{A})$ does indeed 
give rise to a singlet in its ${ }^{1} \mathrm{H}$ NMR spectrum, at $\delta 7.43$ (see Figure 2.12). It is clear, however, that this spectrum cannot belong to triarylmethane 2.2. The triarylmethane should contain only two doublets, each integrating for two protons, as the pyrimidine groups in $\mathbf{2 . 2}$ are identical. Product A, however, appears to possess two non-identical pyrimidine rings, based on the presence of four doublets in the aromatic region of the spectrum. Two separate peaks, which can just be resolved, at $\delta 2.43$ and 2.44 (not shown in the Figure) were assigned to the thiomethyl groups and help to confirm the non-identical nature of the pyrimidine rings. The AMX splitting pattern due to the 2,3-substituted pyridine ring is also clearly visible.

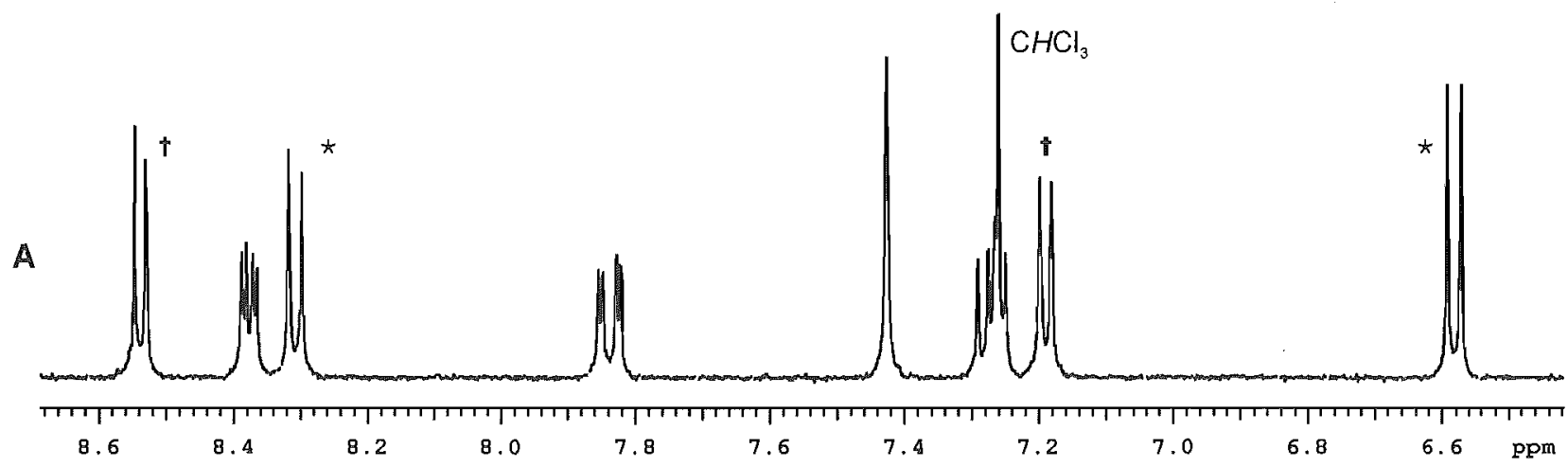

Figure 2.12 Aromatic region of the $300 \mathrm{MHz}{ }^{1} \mathrm{H}$ NMR spectrum of $\mathrm{A}$. Stars and crosses indicate pyrimidine signals that are coupled to each other.

Mass spectral analysis gave the surprising result that $\mathbf{A}$ had the molecular formula $\mathrm{C}_{16} \mathrm{H}_{14} \mathrm{ClN}_{5} \mathrm{OS}_{2}$ and thus, was simply a rearranged isomer of the starting material 2.3. Application of 2D NMR techniques led to the conclusion that $\mathbb{A}$ had the structure 2.39. The wealth of correlations in the HMBC (see Figure 2.13), particularly from the central methine proton to each of the three aromatic rings, was instrumental in the elucidation of this structure.

The formation of $\mathbf{2 . 3 9}$ from triarylmethanol $\mathbf{2 . 3}$ is certainly an interesting transformation. However, before any mechanisms that may lead to 2.39 are discussed, the identity of the other products from the TFA/TES reaction will be addressed. 


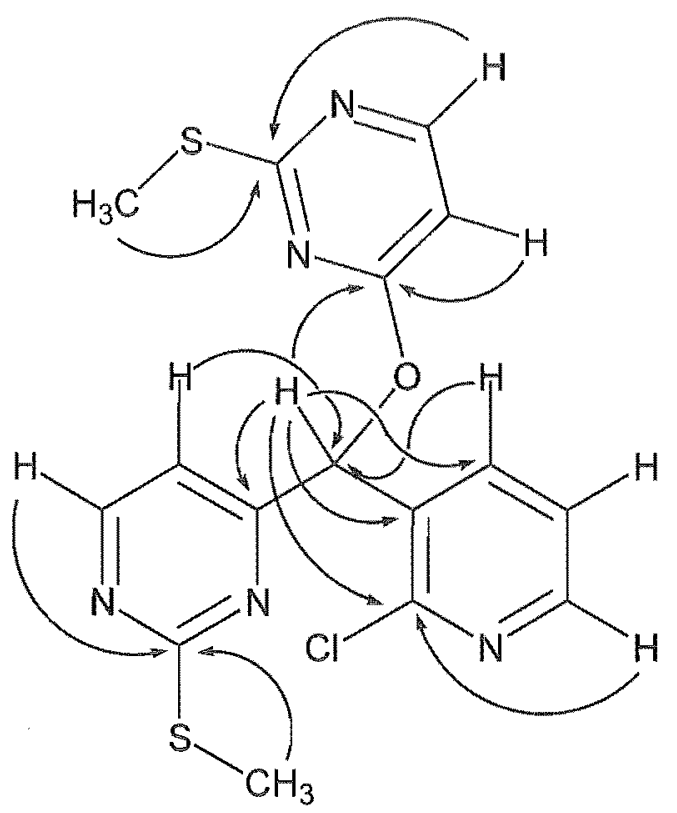<smiles>COc1nccc(C(Oc2ccnc(SC)n2)c2cccnc2Cl)n1</smiles>

$2.39(\mathrm{~A})$

Figure 2.13 Important HMBC correlations for $2.39(\mathrm{~A})$.

The aromatic regions of the ${ }^{1} \mathrm{H}$ NMR spectra of the three remaining products $\mathbf{B}, \mathbf{C}$ and $\mathbf{D}$ are displayed in Figure 2.14. It is immediately apparent that none of these products corresponds to the desired triarylmethane 2.2. Yet these spectra are intriguing, in that they are all consistent with what would be expected for the variolin core structure 2.1. It would appear that the triarylmethane may have been formed and then reacted further to produce the pyridopyrrolopyrimidine core. In each of the three spectra, two separate thiomethyl signals (which appear between 2 and $3 \mathrm{ppm}$ ) were seen, in addition to two pairs of doublets in the aromatic region (Figure 2.14). This would indicate that for each product, the two pyrimidine units are non-identical. The distinctive AMX coupling due to the pyridine ring is also seen for each product. 

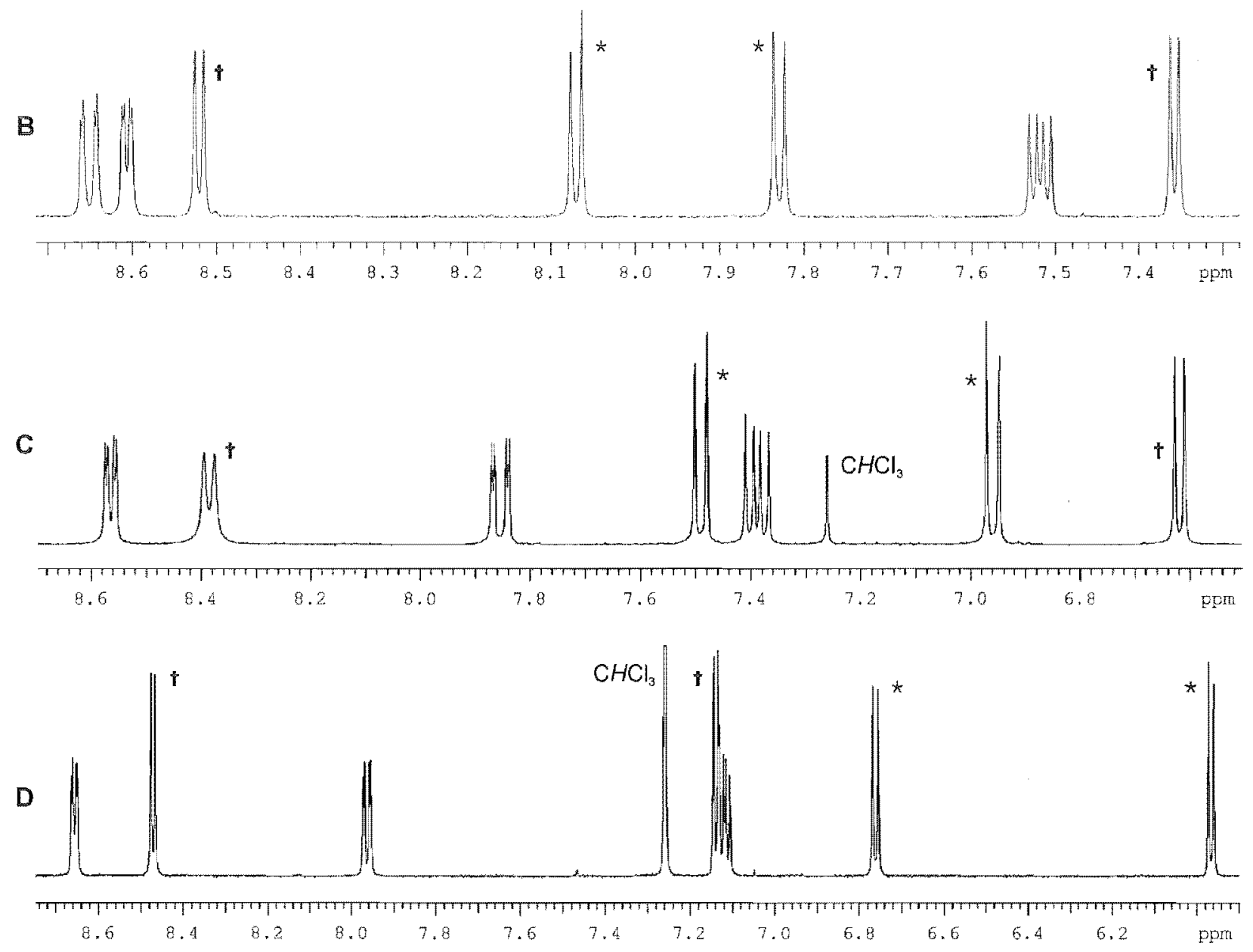

Figure 2.14 Aromatic regions of the ${ }^{1} \mathrm{H}$ NMR spectra of three compounds $\mathbf{B}, \mathbf{C}$ and $\mathbf{D}$ isolated from the TFA/TES reaction. Stars and crosses indicate pyrimidine signals that are coupled to each other.

Mass spectrometry revealed that two of the compounds, $\mathbf{C}$ and $\mathbf{D}$, both had molecular masses of 355 and the same molecular formula of $\mathrm{C}_{16} \mathrm{H}_{13} \mathrm{~N}_{5} \mathrm{OS}_{2}$. This corresponds to a loss of $\mathrm{HCl}$ from the starting material 2.3, suggesting that they may have cyclised to form the pyrido[3',2:4,5]pyrrolo[1,2-c]pyrimidine system. Apparently, however, neither had been deoxygenated. The other compound, $\mathbf{B}$ gave a molecular ion of 339 with the molecular formula $\mathrm{C}_{16} \mathrm{H}_{13} \mathrm{~N}_{5} \mathrm{~S}_{2}$, which is exactly that required by the variolin core structure 2.1 . 
All the NMR data obtained for $B$ is consistent with this structure. With the aid of HSQC and HMBC experiments, nearly all of the ${ }^{1} \mathrm{H}$ and ${ }^{13} \mathrm{C}$ signals were able to be assigned. However, it was unclear which pair of doublets (starred or crossed in Figure 2.14B) belonged to which pyrimidine ring. The answer was found by way of a difference NOE experiment. When the doublet at 7.36 ppm was irradiated, three other signals displayed enhancement (Figure 2.15). One was the doublet at $8.52 \mathrm{ppm}$ (to which the doublet at 7.36 is coupled); another was the doublet of doublets at $8.65 \mathrm{ppm}$ (which had already been conclusively assigned to H4); the third was the doublet at $8.07 \mathrm{ppm}$, which belonged to the other pyrimidine ring. Only H5' of 2.1 could exhibit NOEs with three different protons. On the basis of this experiment, the doublet at 7.36 ppm was assigned to $\mathrm{H} 5$ ' of the pendant pyrimidine ring and therefore, the doublet at $8.07 \mathrm{ppm}$ must belong to $\mathrm{H} 6$ of the fused pyrimidine ring. There is obviously some steric crowding around the biaryl linkage, rendering $\mathrm{H} 4$ and $\mathrm{H} 6$ of the pyridopyrrolopyrimidine core close in space to H5'. Assignment of the other pyrimidine signals (in both the ${ }^{1} \mathrm{H}$ and ${ }^{13} \mathrm{C}$ spectra) followed without difficulty (see Figure 2.16).
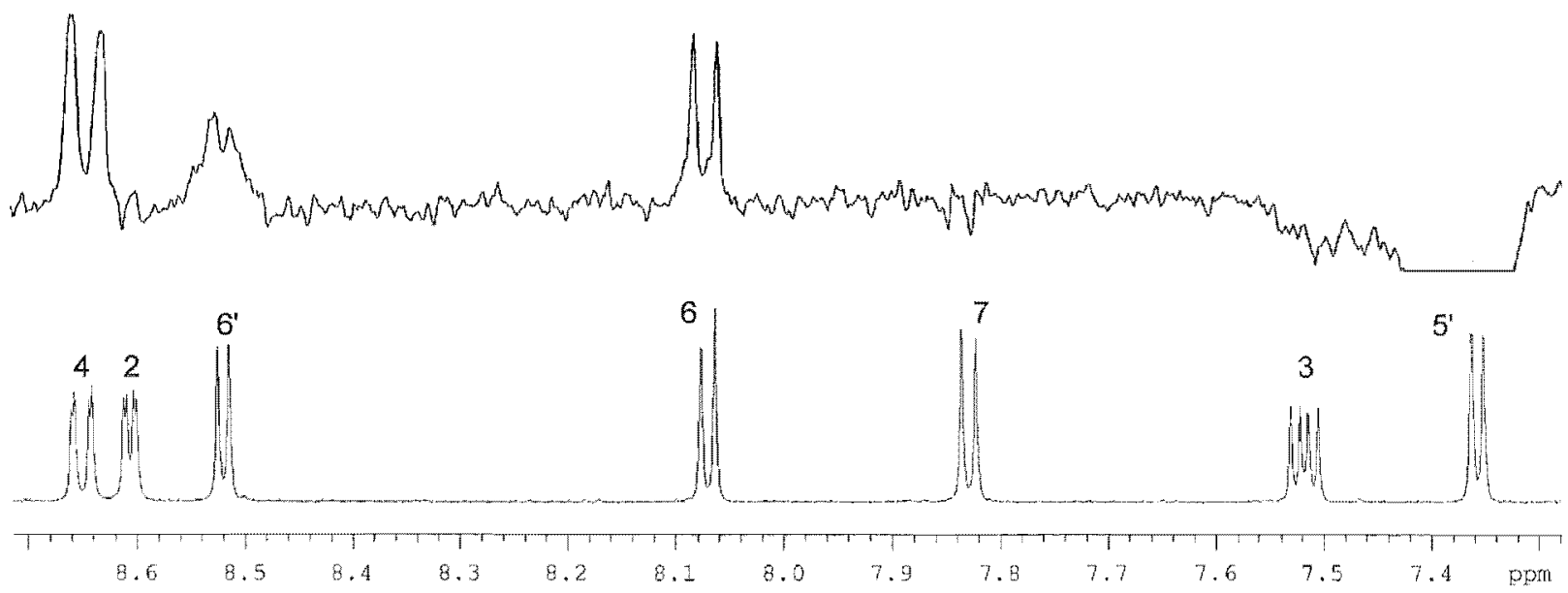

Figure 2.15 NOE irradiation of H5' of 2.1, giving enhancement of three other signals. 


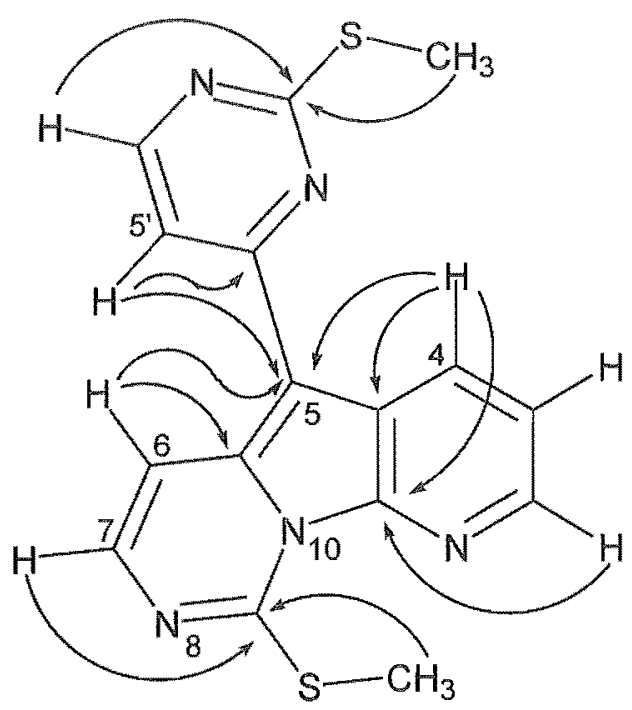

a

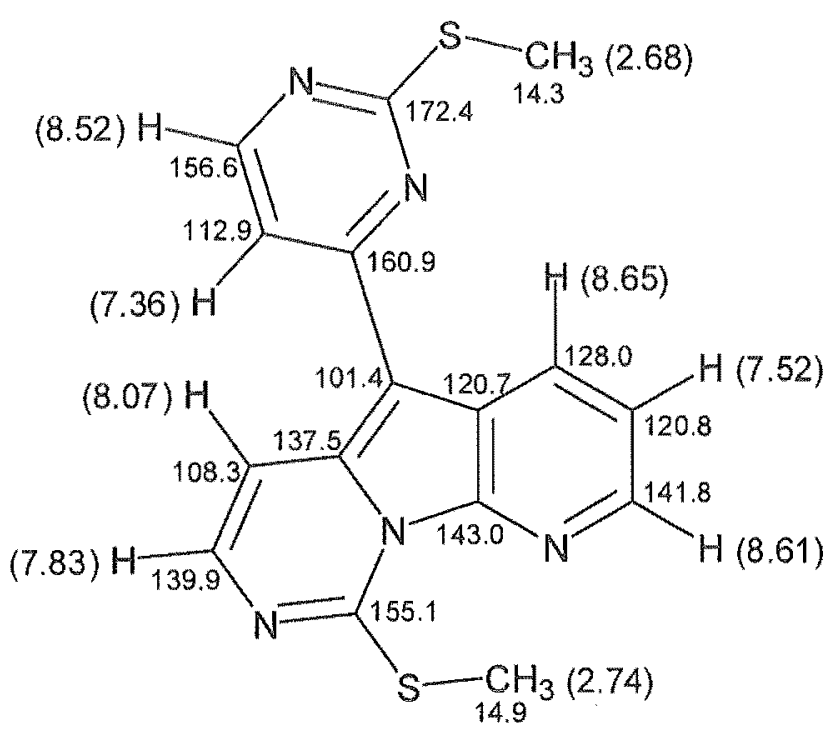

b

Figure 2.16 (a) Important HMBC correlations that helped to confirm the identity of $\mathbf{B}$ as 2.1 ; (b) ${ }^{1} \mathrm{H}$ and ${ }^{13} \mathrm{C}$ NMR chemical shift values for 2.1 .

The ${ }^{1} \mathrm{H}$ NMR chemical shifts of the fused pyrimidine ring are most unusual as $\mathrm{H} 6$ is more downfield than $\mathrm{H} 7$ (which is $\alpha$ to the nitrogen). Compared to the precursor 2.3 , the signal for $\mathrm{H} 6$ has moved downfield by almost $0.7 \mathrm{ppm}$, while the signal for $\mathrm{H} 7$ has moved upfield by just over $0.7 \mathrm{ppm}$. These substantial shifts can be explained by a combination of electronic and anisotropic effects. Electronically, the fused pyrimidine ring can be regarded as being less aromatic than in $\mathbf{2 . 3}$, an assertion which is reflected in the chemical shift values of the ring. (This assertion will be further substantiated later in this Chapter.) In a normal aromatic pyrimidine, $\pi$-polarisation towards the nitrogens leaves the 4-and 6-positions with less electron density. Consequently, higher chemical shifts are observed in the ${ }^{1} \mathrm{H}$ and ${ }^{13} \mathrm{C}$ NMR spectra for these positions. In 2.1, however, there can be no significant delocalisation of the $\pi$ electrons onto either N8 or N10. This argument explains the lower chemical shift of $\mathrm{H} 7$ (and also C7, which appears 18 ppm lower than in 2.3). Why then does H6 appear so far downfield? This is almost certainly due to its position in relation to the pendant pyrimidine ring. It has been demonstrated that $\mathrm{H}^{\prime}$ promotes significant $\mathrm{NOE}$ enhancement for both $\mathrm{H} 4$ and $\mathrm{H} 6$. Both of these protons, therefore, must spend some time in the deshielding zone of the pendant aromatic ring. It is well-known that protons which lie around the periphery of an aromatic ring experience a greater magnetic field in a NMR magnet than they ordinarily would, due to the induced 
aromatic ring current. ${ }^{81}$ These protons are said to be deshielded and appear at higher chemical shifts than normal. This explains the increase of $0.7 \mathrm{ppm}$ in the chemical shift of H6, when compared to 2.3. Proton $\mathrm{H} 4$ experiences an even greater increase in its chemical shift, appearing $1.4 \mathrm{ppm}$ further downfield than in $\mathbf{2 . 3}$.

The combination of all the spectroscopic data convincingly proves that the pyrido[3',2':4,5]pyrrolo[1,2-c]pyrimidine ring system has indeed been produced. However, it is important to note that while the variolin core $\mathbf{2 . 1}$ was formed in just one step from $\mathbf{2 . 3}$, the yield of purified material was only $5 \%$. If a practical synthesis of the variolins was to be developed, conditions would have to be found that minimised the formation of the other side-products. To do so, it was necessary to identify the remaining products $\mathbb{C}$ and $\mathbf{D}$, and to reflect on how $\mathbf{A}, \mathbb{C}$ and $\mathbb{D}$ may have formed.

As discussed earlier, products $\mathbf{C}$ and $\mathbf{D}$ have identical molecular formulas, $\mathrm{C}_{16} \mathrm{H}_{13} \mathrm{~N}_{5} \mathrm{OS}_{2}$ and differ from 2.1 by a single oxygen. From the ${ }^{1} \mathrm{H}$ NMR spectrum of $\mathbb{C}$, it appears to contain a pyridine unit and two non-identical pyrimidine units (Figure 2.17, following page). In addition, the absence of chlorine in its molecular formula indicates that cyclisation to the pyridopyrrolopyrimidine ring system may have occurred. This was confirmed by the use of $2 \mathrm{D}$ NMR spectroscopy, which allowed the determination of two partial structures-the pyridopyrrolopyrimidine core $\mathbf{2 . 4 0}$ (which is also found in 2.1) and the remaining 2(methylsulfanyl)pyrimidine ring 2.41. However these could not be connected to each other. From the molecular formula, the only element unaccounted for is the oxygen. It is assumed, therefore, that the two fragments must be separated by oxygen, which gives 2.42 as the structure for $\mathbb{C}$. It should be noted that a close structural relationship exists between 2.1 and 2.42 which differ only by the presence of an oxygen between C5 and C4'. Some important HMBC correlations which helped to determine this structure are shown in Figure 2.18a. 
<smiles>CSc1nccc2c(C)c3ccccc3n12</smiles>

2.40<smiles>CSc1ccccn1</smiles>

2.41<smiles></smiles>

2.42 (C)

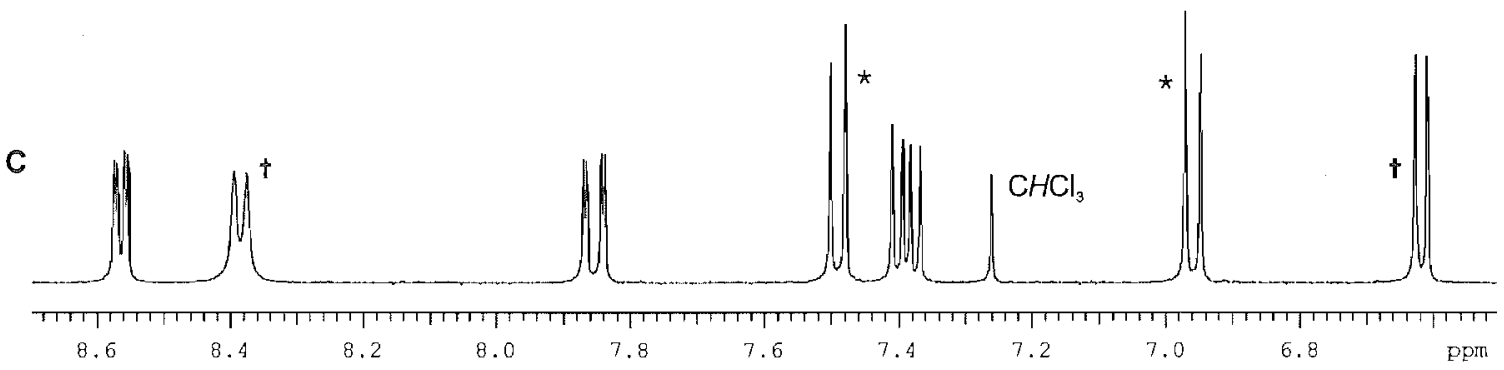

Figure 2.17 Aromatic region of the $300 \mathrm{MHz}{ }^{1} \mathrm{H}$ NMR spectrum of $\mathbb{C}$. Stars and crosses indicate pyrimidine signals that are coupled to each other.

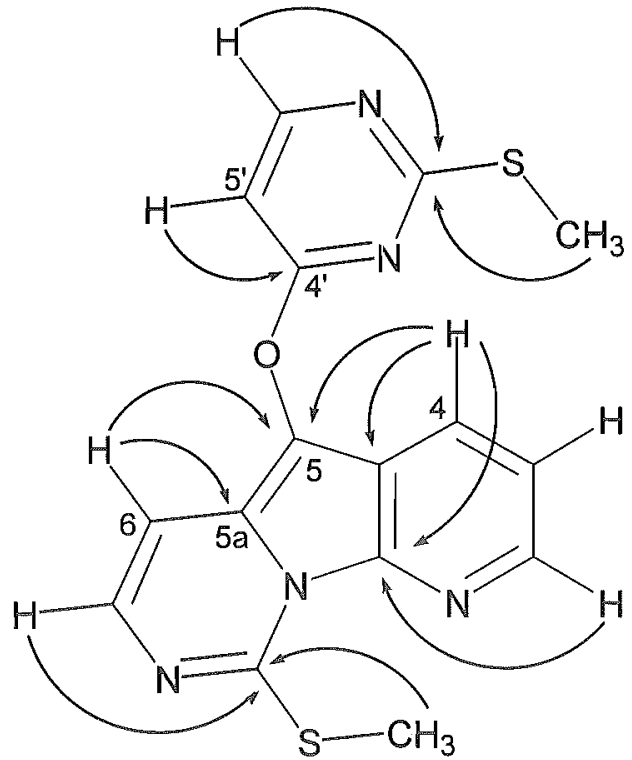

a

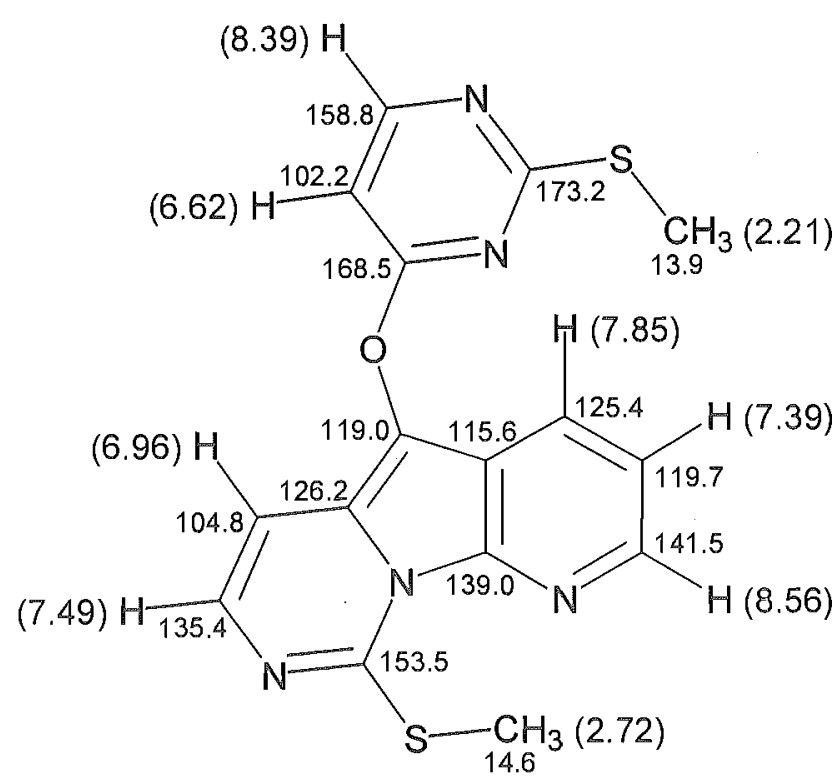

b

Figure 2.18 (a) Important $\mathrm{HMBC}$ correlations for $2.42(\mathbb{C})$; (b) ${ }^{1} \mathrm{H}$ and ${ }^{13} \mathrm{C} \mathrm{NMR}$ chemical shift values for 2.42 . 
The chemical shifts differ from those in 2.1, exactly as would be expected upon insertion of oxygen between $\mathrm{C} 5$ and $\mathrm{C}^{\prime}$ (compare Figure $2.18 \mathrm{~b}$ and Figure $2.16 \mathrm{~b}$ ). In the ${ }^{13} \mathrm{C}$ NMR spectrum, $\mathrm{C} 5$ and $\mathrm{C} 4$ ' both move downfield by 18 and $7 \mathrm{ppm}$, respectively, with the incorporation of an oxygen between them. H5' and C5', on the other hand, move upfield by 0.75 and $11 \mathrm{ppm}$ respectively, due to the $+R$ effect of the oxygen. C5a experiences a similar effect, shifting upfield by $11 \mathrm{ppm}$. It is interesting to compare the chemical shifts of $\mathrm{H} 4$ and $\mathrm{H} 6$ of 2.42 and 2.1. Neither of the protons in the oxygen-containing product experience the anisotropic deshielding of the pendant aromatic ring which was observed in 2.1. Their NMR signals appear upfield by 0.8 and $1.1 \mathrm{ppm}$ respectively, relative to those in 2.1. Neither is any NOE seen between these protons and H5'. Both of these observations are to be expected, if the two ring systems are separated by oxygen. These chemical shift arguments give weighty support to the structure $\mathbf{2 . 4 2}$ for $\mathbf{C}$, which is further substantiated by all of the 2D NMR data (see Figure 2.18).

The final product $(\mathrm{D})$ possesses some rather different spectroscopic features to the former three. For example, one of the pyrimidine rings (starred in Figure 2.19, below) appears to be more alkene-like than aromatic, based on the chemical shifts of the ${ }^{1} \mathrm{H}$ NMR signals (at $\delta 5.96$ and 6.76). A distinctive feature in the ${ }^{13} \mathrm{C}$ spectrum is a signal at $\delta 193$, strongly suggestive of a ketone. The IR spectrum confirms this with a strong peak at $1732 \mathrm{~cm}^{-1}$, which is typical of a 5membered ring ketone. ${ }^{82}$ In the HMBC, correlations from $\mathrm{H} 4$ of the pyridine ring $(87.96)$ and the doublet at $\delta 5.97$ are seen to this carbonyl carbon.

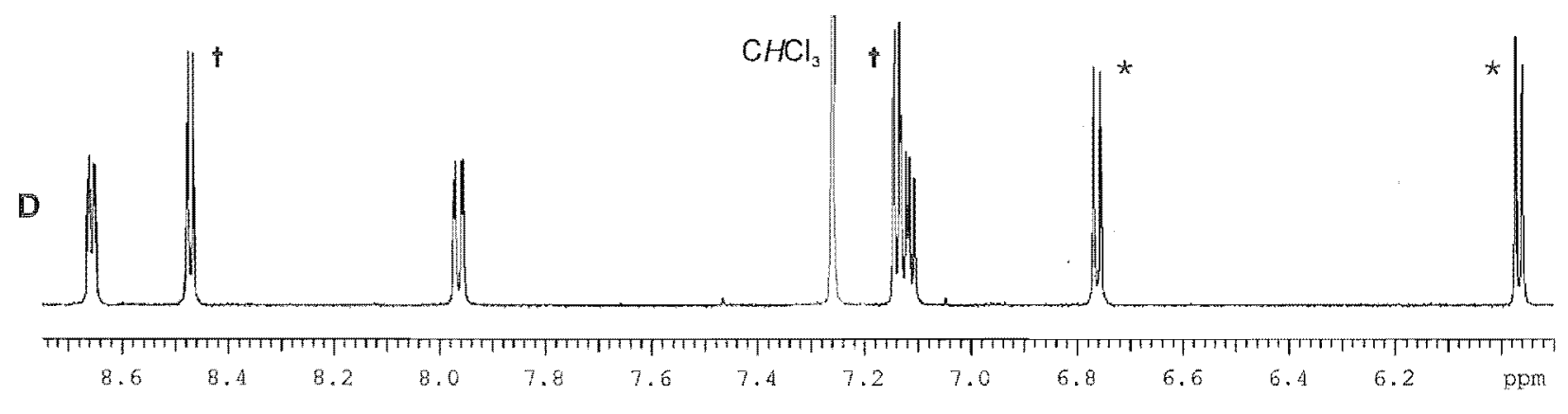

Figure 2.19 Aromatic region of the $500 \mathrm{MHz}{ }^{1} \mathrm{H}$ NMR spectrum of $\mathbb{D}$. Stars and crosses indicate pyrimidine signals that are coupled to each other. 
Consideration of these findings and other data suggests the partial structure 2.43 . This leaves only $\mathrm{C} 5 \mathrm{a}$ with a free valence to accommodate the other pyrimidine ring. This is confirmed in the $\mathrm{HMBC}$, which displays a correlation from $\mathrm{H}^{5}$ ' of this ring $(7.14 \mathrm{ppm})$ to $\mathrm{C} 5 \mathrm{a}$. The structure of is therefore, proposed to be 2.44. Selected H-C correlations observed in the HMBC are shown in Figure 2.20, along with the complete set of ${ }^{1} \mathrm{H}$ and ${ }^{13} \mathrm{C}$ NMR chemical shift values. Further evidence for the structure 2.44 is gained from an NOE between H5' and H6, while no NOE is observed between $\mathrm{H}^{\prime}$ ' and $\mathrm{H} 4$ (as was observed with 2.1). This structure also rationalises the alkene-like ${ }^{1} \mathrm{H}$ NMR signals for the fused pyrimidine ring as no aromatic resonance contributors are possible.

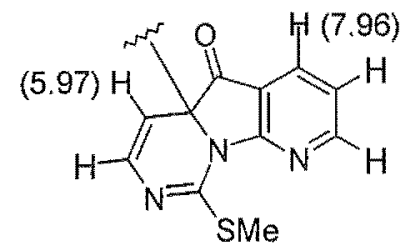

2,43

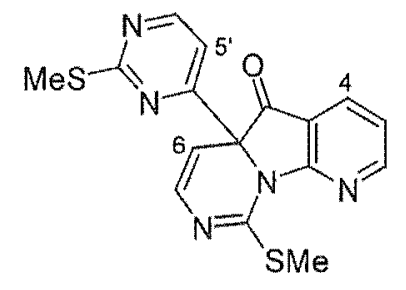

$2.44(D)$

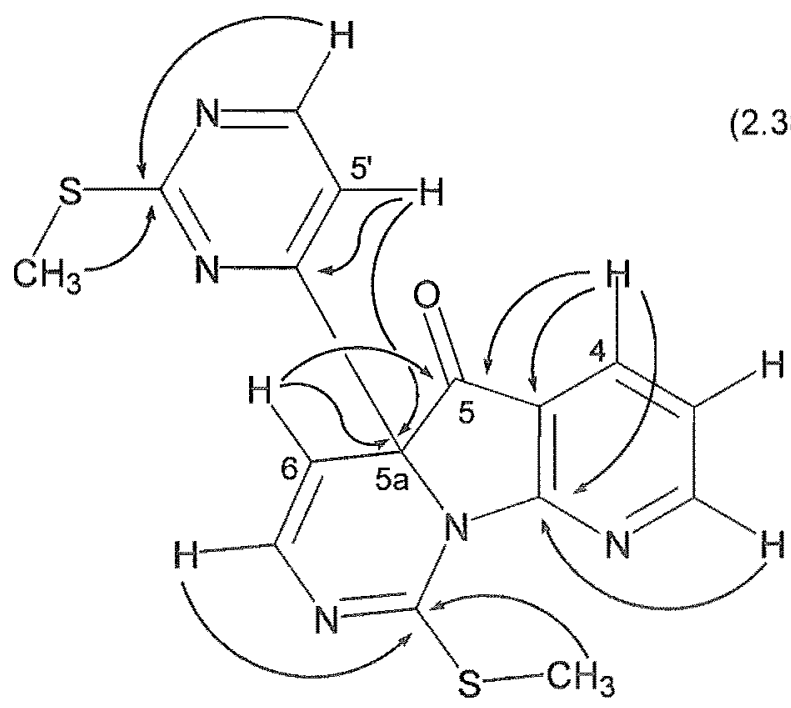

\&

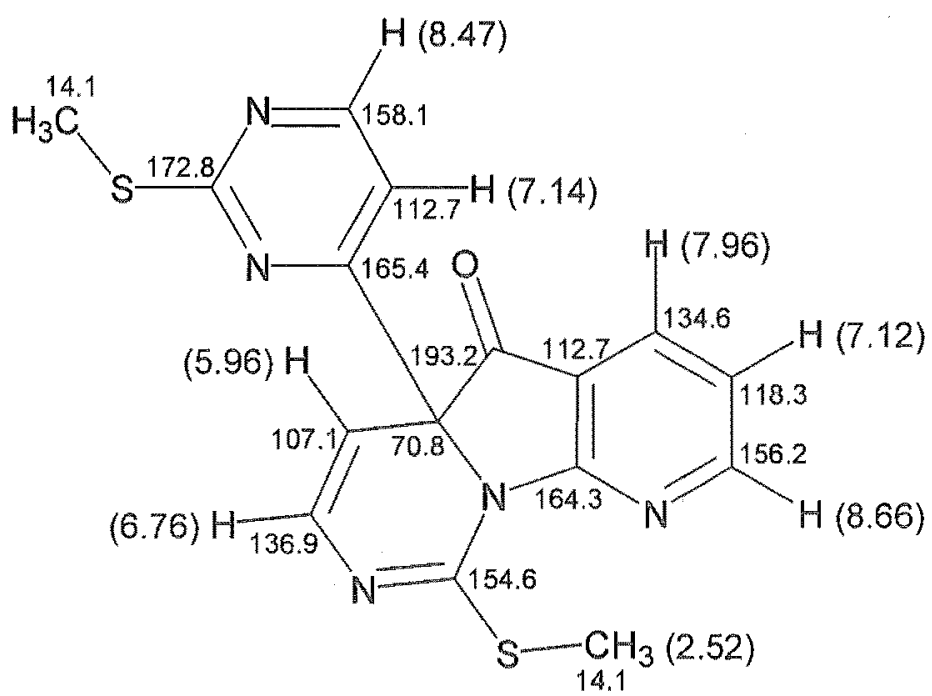

b

Figure 2.20 (a) Important HMBC correlations for 2.44 (D); (b) ${ }^{1} \mathrm{H}$ and ${ }^{13} \mathrm{C}$ NMR chemical shift values for 2.44 . 
Thus, a total of four compounds were isolated and identified from the attempted ionic hydrogenolysis of triarylmethanol 2.3 :

- Product A, 2.39 is a rearrangement product of the starting material.

- Product $\mathbb{B}$ is the desired variolin core structure $2 . \mathbb{1}$, which was thought to have been formed $i n$ situ from triarylmethane $\mathbf{2 . 2}$ (although this intermediate was not observed in the reaction).

- Product C, 2.42 also possesses the pyridopyrrolopyrimidine ring system, although it has retained the oxygen from the starting material.

- Product $\mathbf{D}, 2.44$ is an isomer of $\mathrm{C}$, in which the 2-(methylsulfanyl)pyrimidine ring has undergone a 1,2-shift.<smiles>CSc1nccc(OC(c2ccnc(SC)n2)c2cccnc2Cl)n1</smiles>

$2.39(\mathrm{~A})$<smiles>CSc1nccc(-c2c3cccnc3n3c(SC)nccc23)n1</smiles>

2.1 (B)<smiles>CSc1nccc(Oc2c3cccnc3n3c(SC)nccc23)n1</smiles>

$2.42(\mathrm{C})$<smiles>CSC1=NC=CC2(c3ccnc(SC)n3)C(=O)c3cccnc3N12</smiles>

$2.44(\mathrm{D})$

Several other minor products were formed in the TFA/TES reaction, but only the four discussed were isolated and their structures determined. It is important to note that three out of the four structures possess the pyridopyrrolopyrimidine skeleton found in the variolins. With the mystery of their respective structures solved, the question of how they were formed had to be answered if the formation of the undesired products was to be minimised. 


\subsubsection{Some Mechanisms which may lead to Products 2.1, 2.39, 2.42 and 2.44}

It was initially thought that core structure 2.1 was formed from triarylmethanol 2.3 by a stepwise process involving deoxygenation of 2.3 and reduction of the incipient cation, and then cyclisation of the intermediate triarylmethane 2.2. However the fact that triarylmethane 2.2 was never detected in the reaction mixture, and the presence of the other cyclised compounds 2.42 and 2.44, which still contain oxygen, raised the possibility that an alternative mechanism may be operating. Further evidence for this alternative mechanism was found when it was attempted to cyclise ether 2.39 to form 2.42. This process would be analogous to the suggested cyclisation of triarylmethane $\mathbf{2 . 2}$ to produce the variolin core 2.1. It was thought that, in either case, elimination of $\mathrm{HCl}$ to give the cyclised products 2.1 or 2.42 would be a facile process. However, when 2.39 was heated at reflux in either toluene or TFA, only starting material was returned (Figure 2.21). This was a most-unexpected result, and cast serious doubt on the suggestion that triarylmethane 2.2 was an intermediate in the formation of 2.1 .

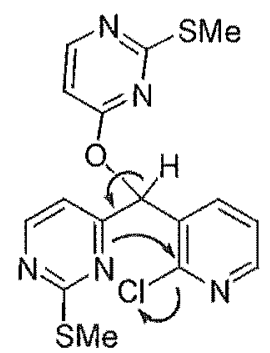

2.39

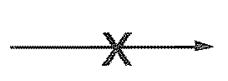

2.42

Figure 2.21 Attempted cyclisation of 2.39 to give 2.42 .

An alternative mechanism can be envisaged in which cyclisation of triarylmethanol 2.3 precedes deoxygenation. This would produce the pyrimidinium salt 2.45 (Figure 2.22). Hydride reduction at $\mathrm{C} 5 \mathrm{a}$ of 2.45 would give 2.46 , which could eliminate $\mathrm{H}_{2} \mathrm{O}$ to produce the variolin core 2.1 . 
<smiles>COc1ncccc1C(O)(c1cccnc1)c1ccnc(SC)n1</smiles>

2.3
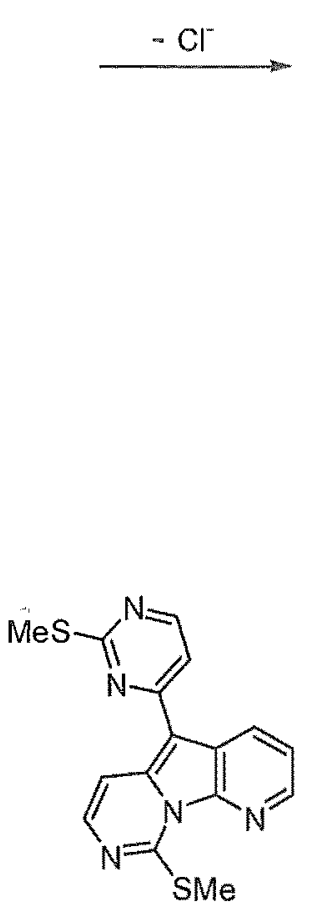

2.1
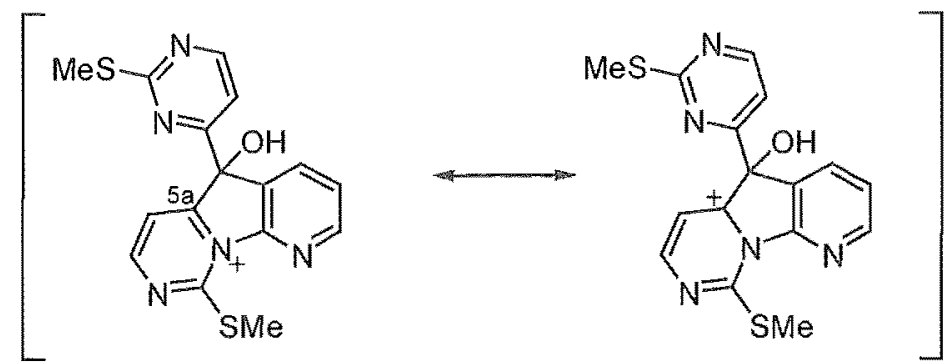

2.45 TES
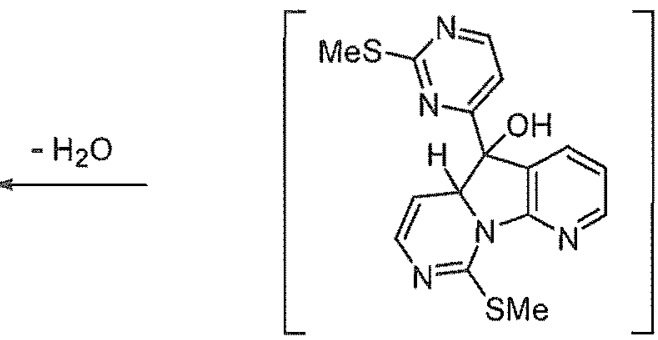

2.46

Figure 2.22 Proposed mechanism of formation of 2.1, where cyclisation precedes deoxygenation.

The same intermediate $\mathbf{2 . 4 5}$ can also account for the formation of the other cyclised products, 2.42 and 2.44. In the case of 2.42, incorporation of oxygen into the 4 '-position of the pyrimidine ring must arise from intramolecular attack of the hydroxyl group at the substituted azomethine bond, as shown in path (a), Figure 2.23. Presumably, protonation of the pyrimidine nitrogen (N3') aids this process. This would produce the strained epoxide $\mathbf{2 . 4 7}$, which could either revert to 2.45 or irreversibly produce 2.42 by breaking the $\mathrm{C}-\mathrm{C}$ bond of the epoxide.

Migration of the pyrimidine ring of intermediate 2.45 (path (b), Figure 2.23) would produce a new cation $\mathbf{2 . 4 8}$, which is stabilised by the attached oxygen. Deprotonation of the hydroxyl group would result in the formation of ketone 2.44 . 

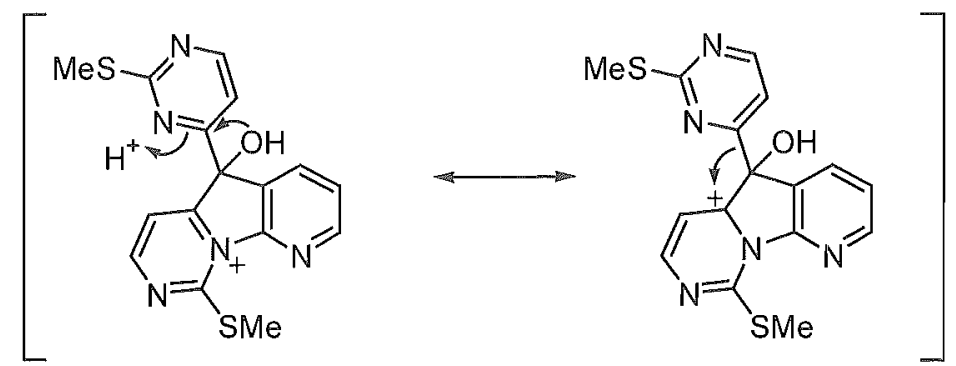

2.45
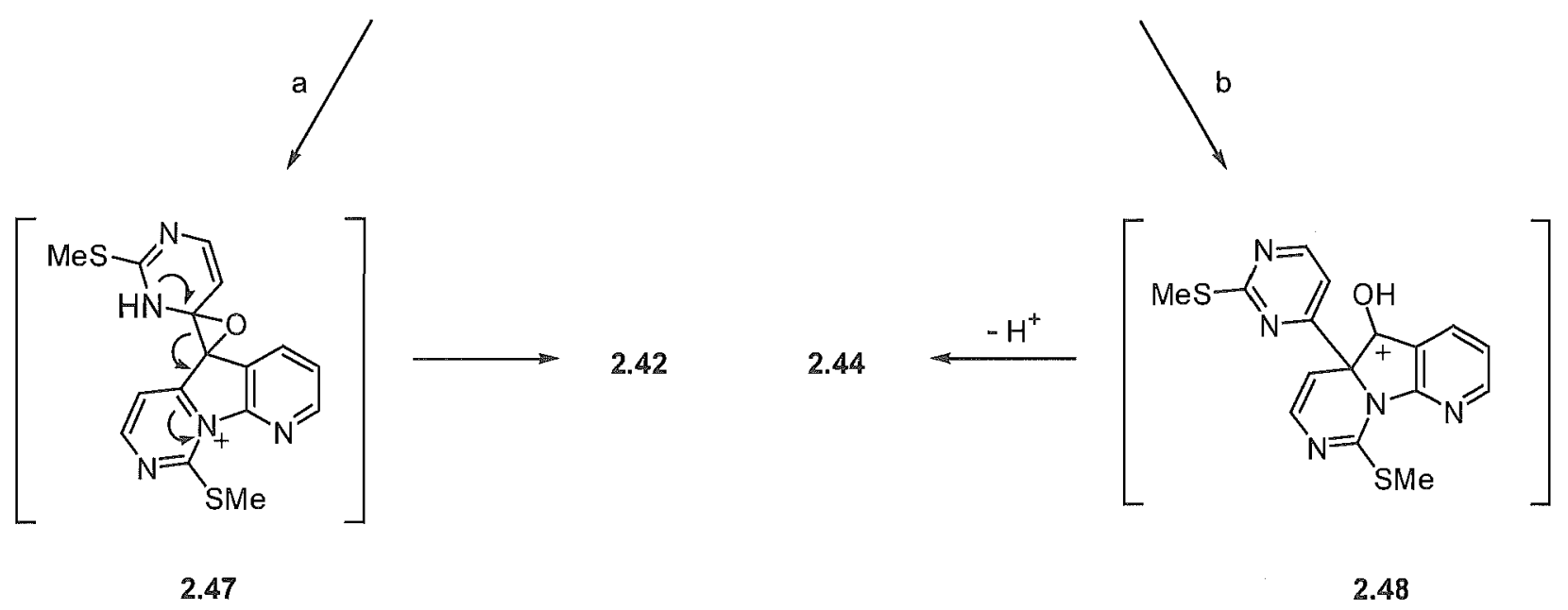

Figure 2.23 Proposed mechanisms of formation of enol ether 2.42 and ketone 2.44 .

This leaves only the uncyclised product 2.39 to consider, with a possible mechanism for its formation shown in Figure 2.24. Starting from the triarylmethanol 2.3, an analogous process to that shown in path (a), Figure 2.23 would account this product.<smiles>CCOCC(c1ccnc(SC)n1)(c1ccnc(SC)n1)c1cccnc1Cl</smiles>

2.3<smiles></smiles>

$\longrightarrow$<smiles>CSc1nccc(OC(c2ccnc(SC)n2)c2cccnc2Cl)n1</smiles>

2.39

Figure 2.24 Proposed mechanism of formation of 2.39. 


\subsubsection{Further Observations on the Mechanism of the TFA/TCS Reaction}

Of the four products isolated from the TFA/TES reaction, the variolin core 2.1 is the only product in which any reductive process involving TES has occurred. The other three products are the result of acid-catalysed processes and should not require TES for their formation. Indeed, when triarylmethanol $\mathbf{2 . 3}$ was heated at reflux in neat TFA for $10 \mathrm{~h}$, the starting material was completely consumed and all three products $2.39,2.42$ and 2.44 were observed in the crude mixture, while no trace of 2.1 could be detected. The following observations are given in an attempt to clarify the processes involved in the TFATES reaction.

- If the reaction with TFA, alone, is subjected to basic workup, ketone 2.44 is isolated as the major product with small amounts of 2.39 and 2.42 also being produced.

- If the reaction mixture is simply concentrated (without workup), ${ }^{1} \mathrm{H}$ NMR analysis of the paste reveals a new product $X$, which is the major component of the mixture (see Figure 2.25). A $\mathrm{CH}_{2} \mathrm{Cl}_{2}$-solution of $\mathrm{X}$ is relatively stable to water, however when exposed to base $(5 \%$ aq $\mathrm{NaHCO}_{3}$ solution), ketone 2.44 is produced, together with small amounts of 2.39 and 2.42 .

- If the reaction is repeated with the inclusion of TES ( 2 equivalents), the same product $\mathrm{X}$ is produced after $10 \mathrm{~h}$ (although not as cleanly). Basic workup, again, produces ketone 2.44 . Small amounts of the variolin core $\mathbf{2 . 1}$ are also present in the crude mixture.

- If the reaction (with the inclusion of TES) is left for $66 \mathrm{~h}, \mathrm{X}$ is not observed (pre-workup). Basic workup gives the variolin core 2.1 as the major product. Ketone $\mathbf{2 . 4 4}$ is not observed. 


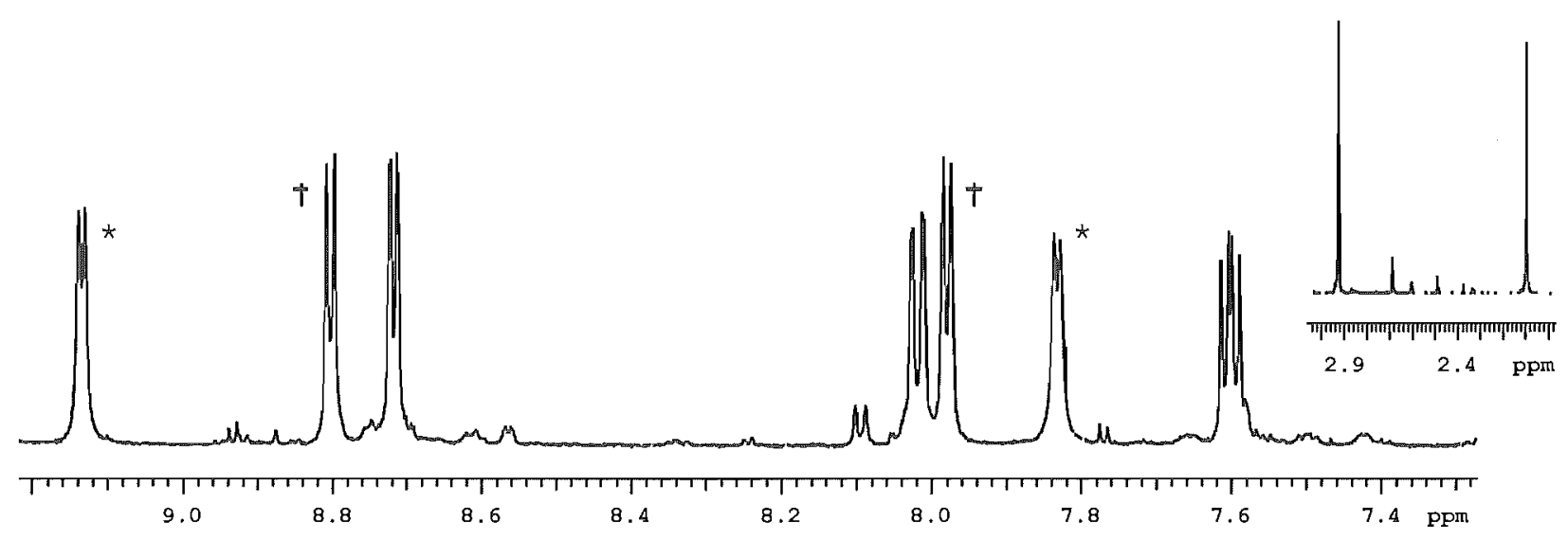

Figure 2.25 $500 \mathrm{MHz}{ }^{1} \mathrm{H}$ NMR spectrum of $\mathrm{X}$. Stars and crosses indicate pyrimidine signals that are coupled to each other.

These observations suggest that the following mechanistic pathways operate:

Heating $\mathbf{2 . 3}$ in TFA initially forms intermediate $\mathrm{X}$, which we propose to have the structure $\mathbf{2 . 4 5}$ (Figure 2.26). This product is stable in acidic solution, and in the presence of TES, it reacts slowly to give the variolin core 2.1. A competing pathway involves rearrangement of $\mathbf{X}$ to enol ether 2.42. If, on the other hand, $X$ is subjected to basic workup, it immediately rearranges to the ketone 2.44. The uncyclised product 2.39 does not form through $\mathbf{X}$, but is rather produced from 2.3 as described earlier. 

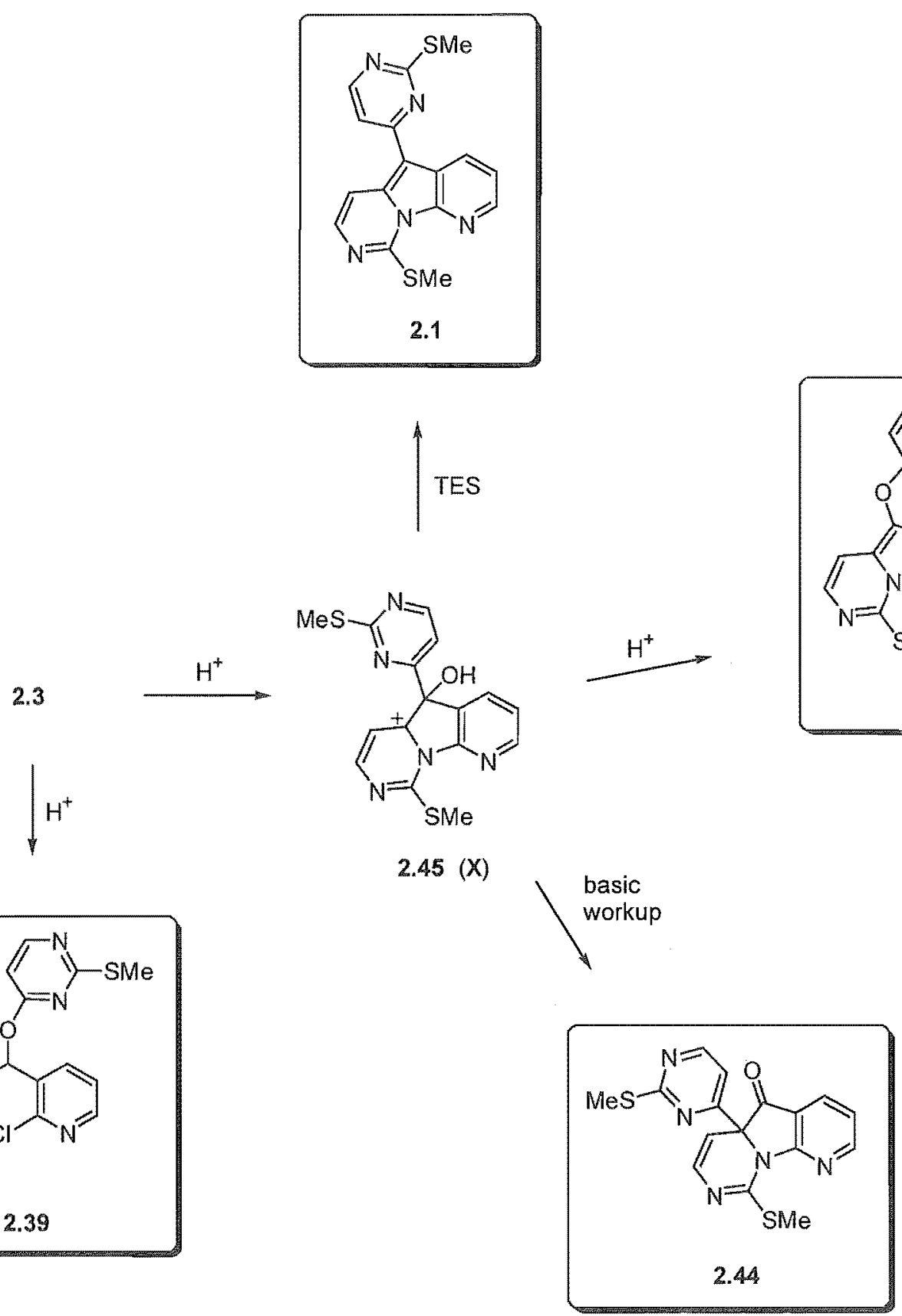

Figure 2.26 Proposed mechanistic pathways leading to products from the TFA/TES reaction.

The foregoing discussion has covered some intriguing aspects of the TFA/TES reaction, including the successful identification of some side-products and possible mechanisms of their formation. Much of the latter is merely speculation. However speculation is useful, if it leads to conclusions which can be tested experimentally. 


\subsubsection{A Stabilised Triarylmethyl Cation?}

It was surmised that the three highly electron-deficient heterocyclic rings make the triarylmethyl cation 2.38 ( $\mathrm{p}$ 48) prohibitively high in energy. Therefore, it was decided to investigate the ionic hydrogenolysis of an alternative triarylmethano1, 2.49. The carbocation produced by ionisation of this alcohol is expected to be considerably stabilised over $\mathbf{2 . 3 8}$, due to the electron-donating anisole ring. If the cation could be produced, reduction with TES would produce a triarylmethane $\mathbf{2 . 5 0}$ (Figure 2.27). This compound could be used to ascertain whether the cyclisation of a triarylmethane to the pyridopyrrolopyrimidine ring system 2.51 is achievable.<smiles>COc1ccc(C(O)(c2ccnc(SC)n2)c2cccnc2Cl)cc1</smiles>

2.49<smiles>COc1ccc(C(c2ccnc(SC)n2)c2cccnc2Cl)cc1</smiles>

2.50

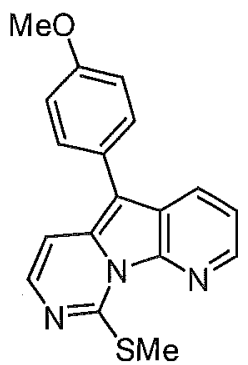

2.51

Figure 2.27 Proposed use of an electron-rich triarylmethanol for the formation of a pyridopyrrolopyrimidine system.

Triarylmethanol 2.49 was readily produced by reaction of 4-methoxyphenyllithium with the biaryl ketone $\mathbf{2 . 1 1}$ (Scheme 2.5). When this was heated with TES (1.2 equivalents) in excess TFA, a yellow product was cleanly produced, which was clearly neither triarylmethane 2.50 nor the pyridopyrrolopyrimidine compound 2.51. MS and NMR analysis led to the identification of the product as 2.52. Slow evaporation of a hexanes solution of $\mathbf{2 . 5 2}$ gave green needles, which were analysed by X-ray crystallography. The results from the diffraction analysis confirmed the structure of 2.52 (see Figure 2.28). 


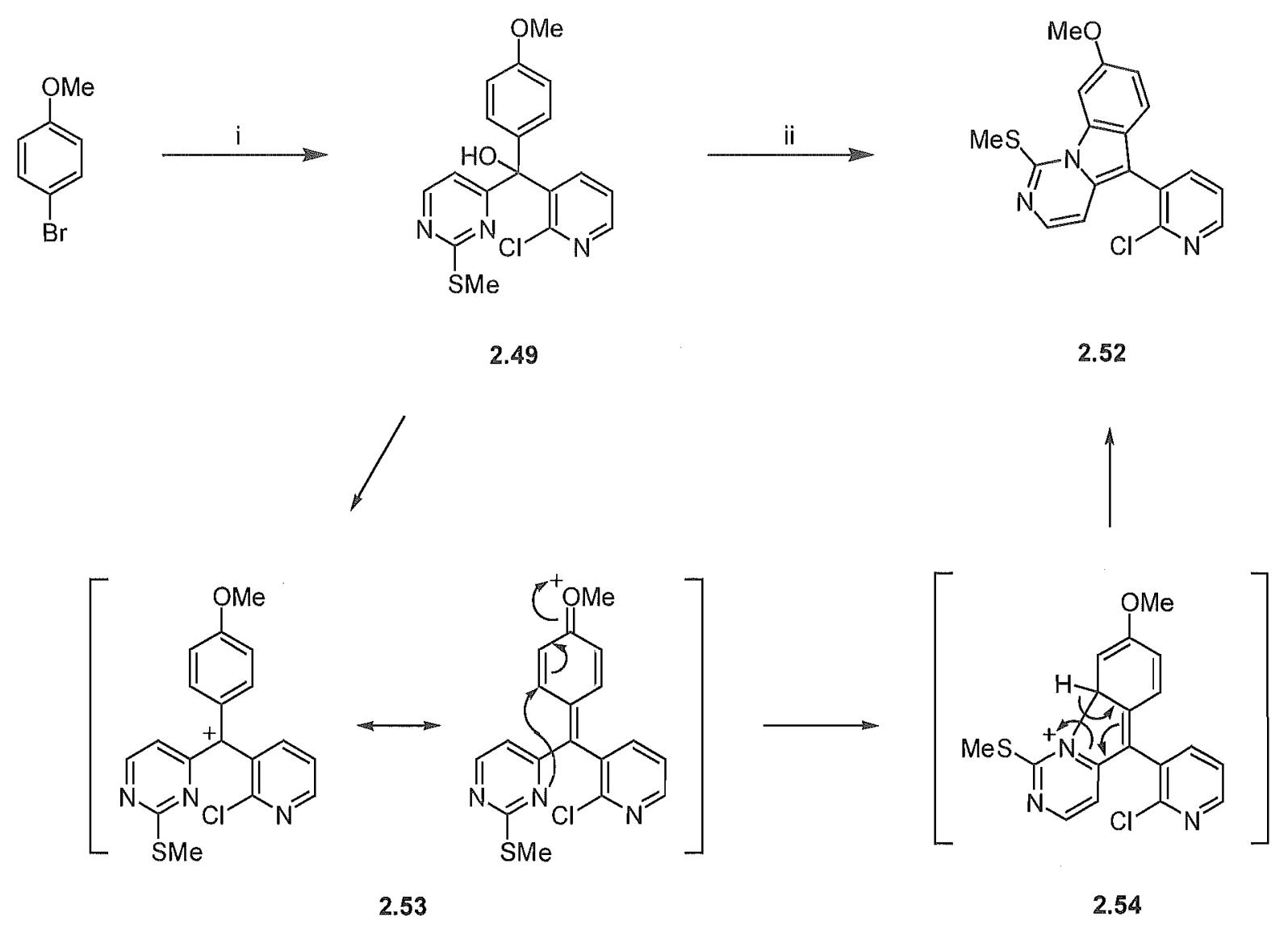

Scheme 2.5 Reagents and yields: (i) BuLi (1 equiv)/THF $/-78^{\circ} \mathrm{C} / 20 \mathrm{~min}$, then 2.11 ( 1 equiv) $/-97^{\circ} \mathrm{C}$ to rt (45\%); (ii) TES (1.2 equiv)/TFA/reflux (53\%).

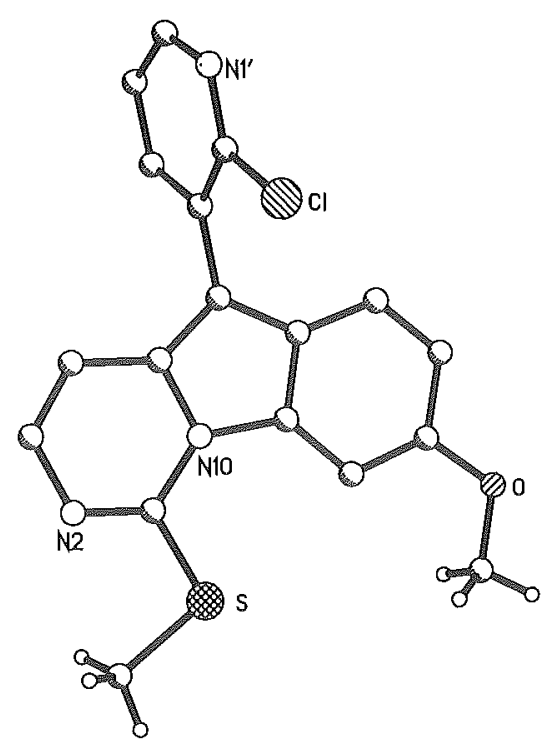

Figure 2.28 X-ray crystal structure of 2.52. Aromatic protons have been omitted for clarity. 
An explanation as to how this interesting structure might be formed is shown in Scheme 2.5. It is believed that the electron-donating anisole ring does, indeed, allow the formation of a stabilised carbocation 2.53. However, before this can be reduced by TES, intramolecular attack of the pyrimidine ring on the anisole ring gives intermediate 2.54 . This requires only the loss of a proton to give the reaction product, formally a pyrimido[1,6- $a]$ indole. Evidently, this model system offers no insights as to how the yield of the variolin core 2.1 might be improved.

\subsubsection{Optimisation of the Yield of Variolin Core Structure 2.1}

It was reasoned that since products $\mathbf{2 . 3 9}, 2.42$ and 2.44 are produced by acid-catalysed processes, their formation might be minimised by decreasing the ratio of acid to reducant in the reaction. A series of experiments was conducted which led to the optimisation of the reaction for the variolin core 2.1. Table 2.1 clearly shows that reducing the amount of acid in the reaction, as well as increasing the proportion of reducing agent, had a beneficial effect on the yield of 2.1. The best results were obtained when 2.3 was heated at $75^{\circ} \mathrm{C}$ for $66 \mathrm{~h}$, with 4 equivalents of TFA and 8 equivalents of TES (Entry 4). Under these conditions, 2.1 was isolated in $34 \%$ yield. The relative proportions of $\mathbf{2 . 1}, \mathbf{2 . 3 9}$ and $\mathbf{2 . 4 2}$ were $140: 5: 13$ (judging by ${ }^{1} \mathrm{H}$ NMR analysis of the crude material). Ketone $\mathbf{2 . 4 4}$ was not detected in the reaction mixture. Reducing the amount of TFA below 4 equivalents did not further improve the yield of 2.1 (Entry 5).

\begin{tabular}{cccc}
\hline Entry & Equiv of TFA & Equiv of TES & Yicld of 2.1 (\%) \\
\hline 1 & 8.3 & 2.0 & 15 \\
2 & 8.2 & 4.0 & 18 \\
3 & 8.2 & 8.0 & 22 \\
4 & 4.3 & 8.1 & 34 \\
5 & 2.0 & 7.8 & 33 \\
\hline
\end{tabular}

Table 2.1 Optimisation of the reaction conditions for the synthesis of 2.1 from 2.3 .

* 2.3 (500 mg) was heated with TFA and TES under argon, at $75{ }^{\circ} \mathrm{C}$ for $66 \mathrm{~h}$. Yields refer to purified products. 
This result was most pleasing and represents a significant improvement on the initial $5 \%$ yield of 2.1. While a yield of $34 \%$ may seem modest, it is important to realise that the variolin core 2.1 has been generated in just three steps from commercially available 4-chloro-2(methylsulfanyl)pyrimidine. Sufficient quantities of 2.1 would now be available to investigate its elaboration to deoxyvariolin B (1.50).

\subsubsection{Summary}

After a considerable amount of effort directed towards the reduction of triarylmethanol $\mathbf{2 . 3}$, the TFA/TES reaction has been determined to be a feasible, and indeed, successful approach to the variolin core 2.1. Triarylmethanol $\mathbf{2 . 3}$, which is produced in just two steps from commercially available materials, can be directly converted to 2.1 in $34 \%$ yield. While only one new bond has been created in this process, the transformation constitutes a significant increase in molecular complexity, by creating a fused tricyclic ring-system from a simple triaryl-substituted methanol precursor. This methodology would hopefully allow the synthesis of deoxyvariolin B (1.50), and should be able to be adapted to provide a synthesis of the natural product variolin B (1.17). 


\subsection{Elaboration of the Variolin Core 2.1 to Deoxyvariolin $\mathbb{B}$}

Completion of the synthesis of deoxyvariolin B (1.50) requires substitution of the thiomethyl groups of 2.1 with amino groups. Generally, such a procedure involves two distinct steps: oxidation of the thiomethyl groups to either sulfoxides or sulfones, followed by displacement with an appropriate amine. Brown and Ford have demonstrated that simple 2-chloro, 2(methylsulfinyl) and 2-(methylsulfonyl)pyrimidines are all of comparable reactivity in nucleophilic displacements with alkyl amines. ${ }^{83}$ An earlier study ${ }^{84}$ showed that 2chloropyrimidines were $>10^{5}$ times more reactive than 2 -(methylsulfanyl)pyrimidines ${ }^{*}$. The corollary inferred $^{83}$ was that oxidation of a (methylsulfanyl)pyrimidine to either the corresponding sulfoxide or sulfone would improve the rate of aminolysis by $>10^{5}$-fold.

\subsubsection{Direct Substitution of Thiomethyl Groups}

In certain cases, however, thiomethyl groups have been directly displaced by amines, or synthetic equivalents, without prior oxidation. For example, Kos and van der Plas have shown that reaction of 2-(methylsulfanyl)purine (2.55) with potassium amide in liquid ammonia gave 2aminopurine (2.56) in 90\% yield (Scheme 2.6). ${ }^{85}$ This reaction, although simple in appearance, proceeds through a ring-opening mechanism in which the amide anion attacks exclusively at the 6-position of the purine. The mechanism was determined by the use of ${ }^{15} \mathrm{~N}$-labelled potassium amide. When position 6 of purine was blocked by a methyl substituent, nucleophilic attack did not occur at any other position. ${ }^{86}$ Both the complex mechanism involved in formation of 2.56 and the absence of nucleophilic attack at the 2-position of 6-methylpurine make this procedure unsuitable for substitution of the thiomethyl groups in 2.1.

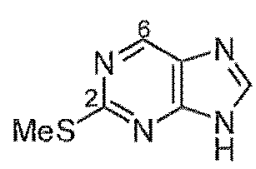

2.55

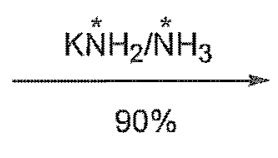

Scheme 2.6

\footnotetext{
"The trivial term thiomethyl is used throughout this thesis, while methylsulfanyl is used for proper names.
} 
Acetamide has also been used in nucleophilic substitutions as a synthetic equivalent for ammonia. For example, the thiomethyl group of 2.57 was displaced in $81 \%$ yield by the acetamide anion (Scheme 2.7). ${ }^{87}$ The amide was deprotected later in the synthesis, in $84 \%$ yield, by heating in aqueous TFA, to complete a total synthesis of the nucleoside antibiotic cadeguomycin (2.58).

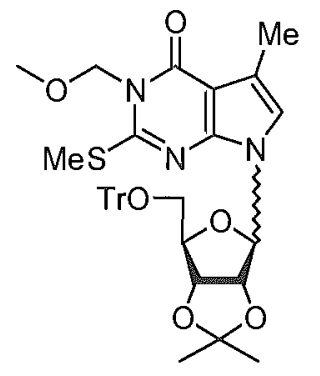

2.57

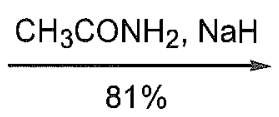

$81 \%$

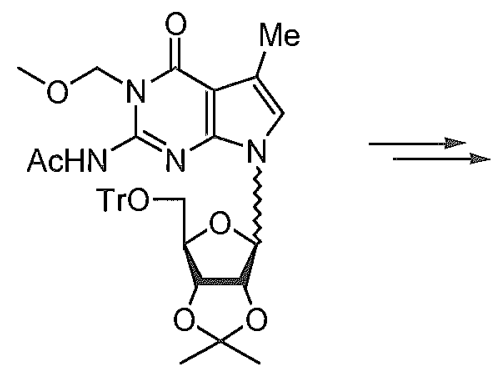

Scheme 2.7

In this reaction, the nucleoside substrate can be considered an extended Michael acceptor due to the $\alpha, \beta-\gamma, \delta$-unsaturated carbonyl group. Its presence may render 2.57 a more reactive electrophile than, for example, the 2-(methylsulfanyl)pyrimidine attached at C5 of the variolin core 2.1. Even so, the reaction required rather harsh conditions, proceeding at $135{ }^{\circ} \mathrm{C}$ in the presence of excess sodium acetamide and absence of a solvent. Attempted application of this methodology to the variolin core 2.1 gave a complicated product mixture. No attempt was made to identify any of the products - it was uncertain whether the pyridopyrrolopyrimidine nucleus had even survived the reaction conditions.

2-(Methylsulfanyl)pyrimidines, or related compounds, have occasionally been substituted with amines or synthetic equivalents under neutral conditions. For example, reaction of 2.59 with excess hydrazine hydrate resulted in substitution of the thiomethyl groups in $71-76 \%$ yield (Scheme 2.8) ${ }^{88}$ The hydrazine functions were subsequently nitrosated to give azides 2.60 , which were later reduced with zinc dust in acetic acid to yield amines 2.61 in $54-58 \%$ yield. 
The same substrates $\mathbf{2 . 5 9}$ were also directly substituted (under acid catalysis) with a variety of anilines to give amines 2.62 .<smiles>[R]c1sc2nc(N[Al])[nH]c(=O)c2c1[R]</smiles>

2.62

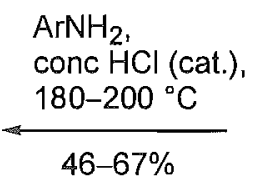

$\mathrm{R}^{1}, \mathrm{R}^{2}=\mathrm{Me}$ or $\mathrm{R}^{1}, \mathrm{R}^{2}=-\left(\mathrm{CH}_{2}\right)_{4}-$<smiles>[R]c1sc2nc([SiH3])[nH]c(=O)c2c1[R]</smiles>

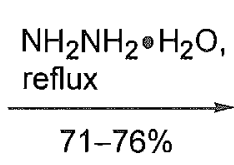<smiles>[R]c1sc2nc(NN)[nH]c(=O)c2c1[R]</smiles>

2.59<smiles>[R]c1sc2nc(N)nc(N)c2c1[R]</smiles>

2.61

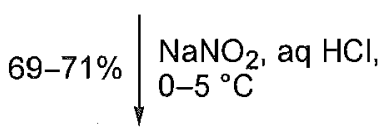

Scheme 2.8

In all of the examples mentioned, the substitutions are performed on fused derivatives of the pyrimidine nucleus. However, the 2-thiohexyl group of pyrimidine 2.63 undergoes direct substitution with a variety of amines at $80{ }^{\circ} \mathrm{C}$ in dioxane (Scheme 2.9). ${ }^{89}$ As there are two sites for substitution, mixtures of products 2.64 and 2.65 were isolated. While substitution of the more activated 4-SMe group occurred more readily, appreciable yields of products 2.65 were obtained. Selective substitution of the thiohexyl group could be achieved when the hexyl S-atom was oxidised to the sulfoxide using $m$-CPBA, prior to aminolysis. 


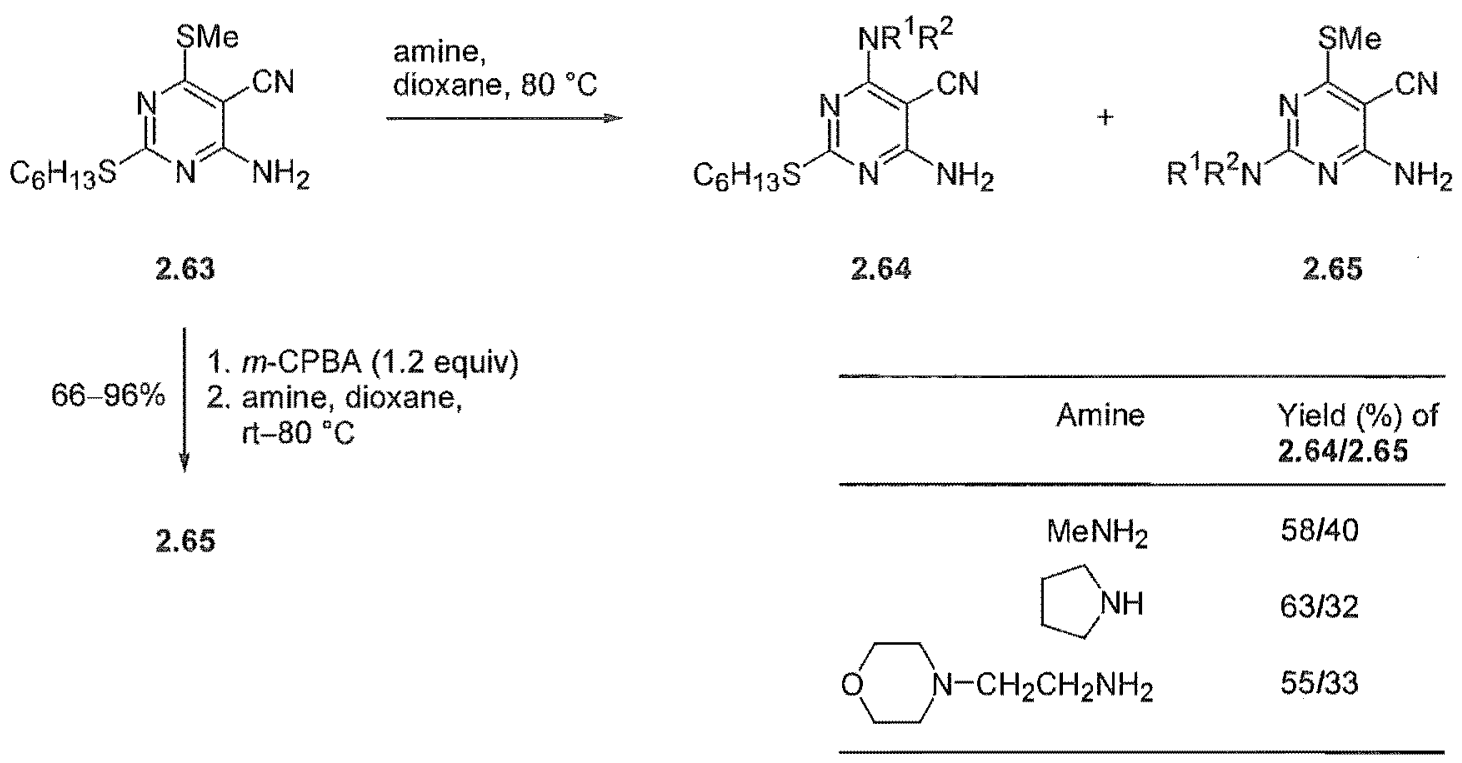

Scheme 2.9

The prospect of introducing both amines into the variolin core 2.1 in a single step, without the need for $S$-oxidation, held significant appeal and thus, pyrrolidine was chosen as a convenient nucleophile to examine this possibility with. Heating of $\mathbf{2 . 1}$ with excess pyrrolidine ( 20 equiv) in dioxane at $80^{\circ} \mathrm{C}$ for $28 \mathrm{~h}$ (as described in reference 89 ) resulted in the clean formation of a new product. Judging from the ${ }^{1} \mathrm{H}$ NMR spectrum, substitution of just one of the thiomethyl groups had taken place. This was readily deduced as only one thiomethyl peak (at $\delta 2.65$ ) was observed, in addition to two signals from the eight methylene protons of the pyrrolidine moiety. It appeared that it was the 9-SMe group which had been replaced, based on the chemical shift differences between the starting material and product. Application of 2D NMR spectroscopy, and comparison with the ${ }^{1} \mathrm{H}$ and ${ }^{13} \mathrm{C}$ chemical shifts of the starting material, confirmed that the product was indeed 2.66, as shown in Scheme 2.10. 


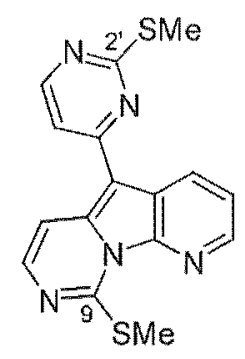

2.1

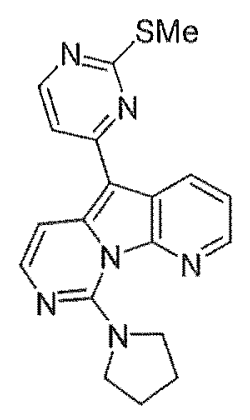

2.66

Scheme 2.10 Reagents and yields: (i) pyrrolidine (20 equiv)/dioxane/80 ${ }^{\circ} \mathrm{C}(50 \%)$

While it was not possible to displace both thiomethyl groups, this result was very encouraging as it meant that nucleophiles should be able to be selectively introduced at C9. Provided that oxidation of the 2'-SMe group was achievable, other substituents could then be introduced at this position, thus enabling a series of related analogues to be synthesised. Efforts to this end are described in Chapter Four. If identical substituents were required at $\mathrm{C} 9$ and $\mathrm{C} 2$ ' (as for deoxyvariolin B and variolin B), the most direct route would entail $S$-oxidation followed by aminolysis at both positions simultaneously. Accordingly efforts were turned towards the oxidation of the thiomethyl groups of the variolin core 2.1 .

\subsubsection{Oxidation/Nucleophilic Substitution of Thiomethyl Groups}

Many systems have been described for the oxidation of sulfides. ${ }^{90}$ In many cases, the reaction can be controlled, allowing isolation of either the sulfoxide, or if excess oxidant and/or higher temperatures are used, the sulfone. In the present case, it should not matter which product was favoured as both have been shown to be of comparable reactivity to amine nucleophiles (see discussion above). Due to the presence of two non-identical thiomethyl groups in 2.1 , several oxidised products were possible. Either of the sulfurs could be oxidised to the sulfoxide or sulfone, in addition to the possibility of both sulfurs being oxidised. Thus, up to eight products were possible. In addition, the chiral nature of the sulfoxides could lead to further diastereomeric products. However, even if several products were obtained, aminolysis of the crude mixture should, in theory, give only one product--a bis-amine. The only requirement, it 
seemed, was that the 2'-SMe group be oxidised, at least to the sulfoxide, as it has already been shown that the 9-SMe group is readily displaced without requiring oxidation.

A cheap and convenient reagent which has been extensively used in the oxidation of thiomethyl groups is Oxone ${ }^{(B)}$, which contains $49.5 \%$ potassium peroxymonosulfate $\left(\mathrm{KHSO}_{5}\right)$ as the active oxidant. When 2.1 was treated with 6 equiv of Oxone ${ }^{\text {(B) }}$ in THF/water at room temperature, the starting material was consumed after $30 \mathrm{~min}$. The major component of the mixture was the sulfoxide 2.67, as determined by ${ }^{1}$ H NMR spectroscopy and MS analysis (Scheme 2.11).

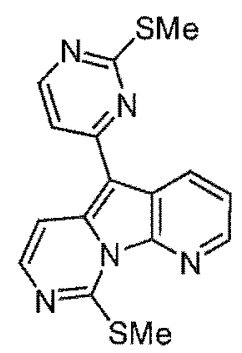

2.1
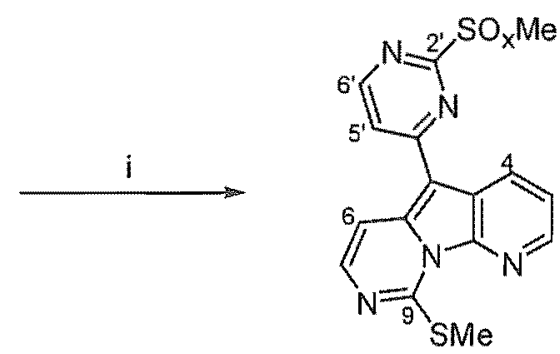

$2.67 x=1$

$2.68 x=2$

Scheme 2.11 Reagents and yields: (i) Oxone ${ }^{\text {(1) }}$ (6 equiv)/aq THF/rt (2.67 24\%).

In this situation, the chemical shifts of the $\mathrm{SO}_{x} \mathrm{CH}_{3}$ signals $(\mathrm{x}=0,1$ or 2$)$ are diagnostic of the oxidation level of the sulfur. In 2.1, the thiomethyl groups give rise to singlets at $\delta 2.68$ and 2.74, while sulfoxide $\mathbf{2 . 6 7}$ displayed peaks at $\delta 2.74$ and 3.03. The second value is fairly typical of 2-(methylsulfinyl)pyrimidines. ${ }^{91}$ The signals for $\mathrm{H}^{\prime}$ and $\mathrm{H} 6^{\prime}$ display similar shifts, moving downfield by 0.36 and $0.25 \mathrm{ppm}$, respectively, upon oxidation of the sulfide (Figure 2.29 ). Conclusive evidence for the position of oxidation was obtained from the HMBC. A correlation from the sulfoxide protons $(\delta 3.03)$ to $\mathrm{C} 2{ }^{\prime}(\delta 173.6)$ showed that it must be the pendant pyrimidine ring that has been oxidised. The analogous position of the fused pyrimidine ring (C9) has a quite different chemical shift of $\delta$ 155.6. As with 2.1, H5' showed strong NOEs to three other protons: $\mathrm{H} 6$ ', $\mathrm{H} 4$ and $\mathrm{H} 6$. 


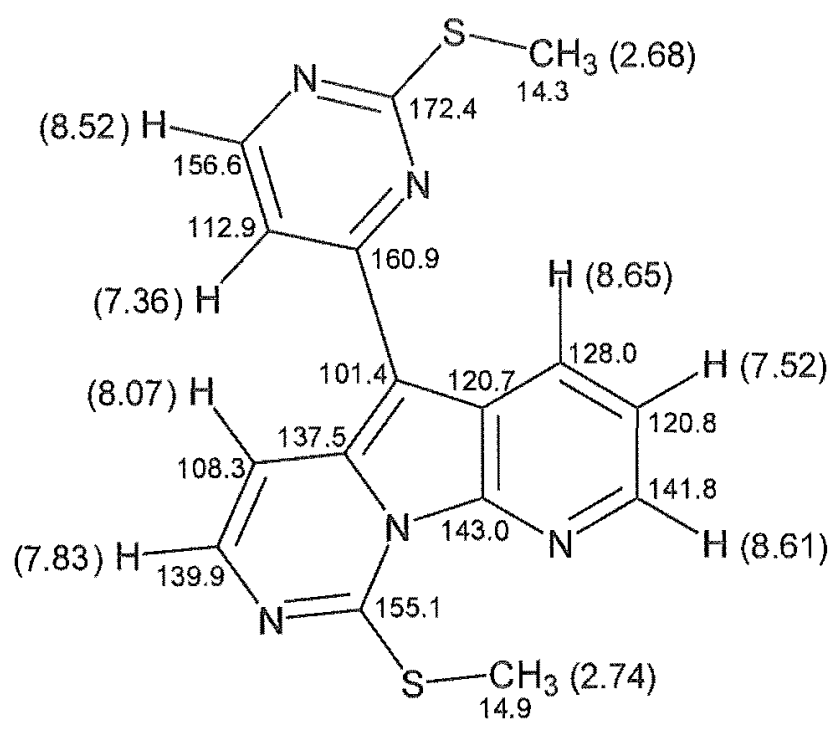

$a$

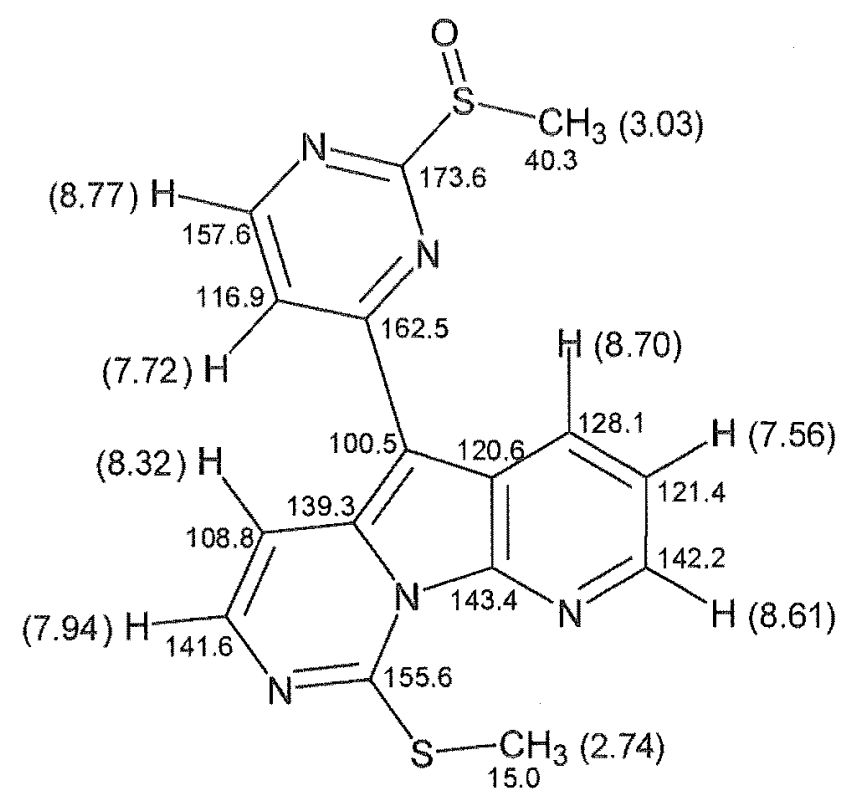

b

Figure 2.29 ${ }^{1} \mathrm{H}$ and ${ }^{13} \mathrm{C}$ NMR chemical shifts of (a) the variolin core structure 2.1 and (b) sulfoxide 2.67 .

Closer examination of the ${ }^{1} \mathrm{H}$ NMR spectrum of the crude reaction mixture revealed a small singlet at $\delta 3.41$, which probably corresponds to the sulfone 2.68 (Scheme 2.11). This $\delta$-value is typical of the $\mathrm{SO}_{2} \mathrm{CH}_{3}$ protons of 2-(methylsulfonyl)pyrimidines. ${ }^{47,91}$ A trace amount of oxidation of the 9-SMe group to the sulfoxide may be indicated by a very small peak at $\delta 3.22$.

This selectivity in oxidation is interesting and can be explained with a mechanistic understanding of sulfide oxidation. Most sulfide oxidising agents (including Oxone ${ }^{(3)}$ ) are electrophilic in nature. The mechanism of oxidation involves nucleophilic attack by sulfur on the oxidising agent, as shown in Figure 2.30. Such a mechanism explains why sulfoxides are oxidised less readily than the comparatively electron-rich sulfides (and why they can often, therefore, be isolated before further oxidation to the sulfone occurs). In 2.1 , the non-bonding electrons of the sulfur at $\mathrm{C} 9$ must be delocalised, to some extent, within the fused pyrimidine ring of the variolin core-they are less available, therefore, for oxidation by an electrophilic oxidant than those of the sulfur at $\mathrm{C} 2$ '. 


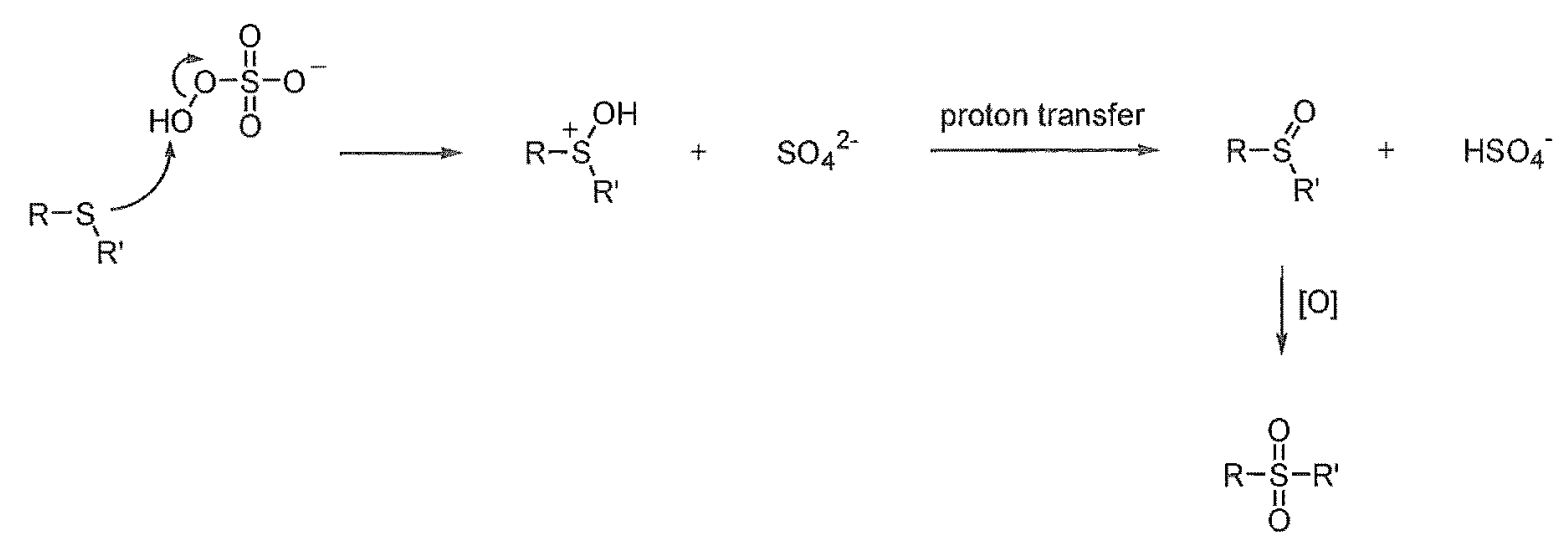

Figure 2.30 Mechanism of sufide oxidation by peroxymonosulfate.

The low yield of 2.67 (24\%) was due, in part, to the difficulties experienced in chromatography. These products tend to stick to silica and consequently elute over many fractions, making separation from other products very difficult. This seems to be a general phenomenon for compounds derived from the variolin core 2.1. For example, flash chromatography of 2.1, following the guidelines given by Still and coworkers, ${ }^{92}$ caused the product to elute over more than 15 fractions. Three consecutive purifications were required to obtain the bulk of the product in a pure state.

In the case of 2.67 , however, the low yield was not solely attributable to losses from chromatography. A yellow precipitate was observed in the reaction, which was not soluble in either aqueous or halogenated solvents. Longer reaction times led to increased amounts of precipitate, while shorter reaction times left significant amounts of starting material unreacted. It was speculated that the insoluble material may be a product of pyridine $N$-oxidation.

While it was encouraging to find that the use of Oxone ${ }^{\circledR}$ allowed the selective oxidation of the 2'SMe group, this reagent did not provide the clean oxidation that is typical of most sulfides and was therefore not suitable for the present synthesis. One drawback of the Oxone ${ }^{\mathbb{B}}$ reagent (other than the poor results of the reaction) was that it required water-soluble solvents $-\mathbf{2 . 1}$ is only modestly soluble in such solvents. As 2.1 has good solubility in $\mathrm{CH}_{2} \mathrm{Cl}_{2}$ and chloroform, it was decided to find a homogeneous oxidant that performs well in these solvents. 
The obvious choice was $m$-chloroperoxybenzoic acid ( $m$-CPBA), a strong (albeit, not very selective) oxidant which is routinely used in $\mathrm{CH}_{2} \mathrm{Cl}_{2}$ or chloroform. When 2.1 was stirred at room temperature with 4 equivalents of $m$-CPBA, a yellow precipitate formed almost immediately. As with the precipitate encountered earlier, this was insoluble in both aqueous and organic solvents. Suspecting that $N$-oxidation may once again have taken place, the reaction was repeated, but using only 1.9 equivalents of $m$-CPBA at $-15^{\circ} \mathrm{C}$. This time, no yellow precipitate was produced, and TLC analysis indicated the formation of several products of greater polarity than 2.1. The major product appeared to be the bis-sulfoxide 2.69 , judging from the ${ }^{1} \mathrm{H}$ NMR spectrum of the crude mixture. As with mono-sulfoxide 2.67, the signals for the SMe protons had moved downfield significantly, appearing at $\delta 3.03$ and 3.23 for 2.69. As 2.69 contains two sulfoxide groups, the molecule can exist in four diastereomeric forms (two pairs of enantiomers). The diastereomers should give rise to slightly different signals in the ${ }^{1} \mathrm{H}$ NMR spectrum. Indeed, ${ }^{1} \mathrm{H}$ NMR analysis showed that the compound is a 1:1 mixture of diastereomers. In some cases, the signals for the two diastereomers can almost be resolved, while other signal pairs completely coincide.

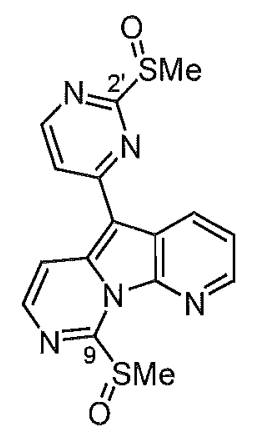

2.69

Closer examination of the ${ }^{1} \mathrm{H}$ NMR of the reaction mixture indicated the presence of monosulfoxide 2.67. In addition, a very small peak was observed at $\delta 3.4$, which probably indicates a small amount of sulfone, as observed in the Oxone ${ }^{\circledR}$ oxidation. It appears then, that as with Oxone $^{\circledR}$, the 2'-SMe group is oxidised by $m$-CPBA to the sulfoxide faster than the 9-SMe group. However, the selectivity differs from that seen with Oxone ${ }^{\circledR}$, in that the remaining thiomethyl group (9-SMe) is then oxidised to the sulfoxide, in preference to oxidation of $2^{2}-\mathrm{SOMe}$ to the sulfone. 
It should be noted that the only products observed were those of $S$-oxidation, with none of the insoluble precipitate seen earlier being formed. Thus, the stage is set for the introduction of the amino groups. It was decided to use a protected amine rather than ammonia itself, even though this would require at least one extra step in the synthesis (for amine deprotection). The desired product of this reaction, bis-protected amine 2.70 , would be more easily handled than deoxyvariolin $\mathrm{B}(\mathbf{1 . 5 0})$, both in terms of polarity and solubility. Variolin B was found to be extremely insoluble in all solvents other than $\mathrm{DMSO}^{32 \mathrm{a}}$ and deoxyvariolin $\mathrm{B}$ was expected to exhibit similar properties. $p$-Methoxybenzyl (PMB) groups were chosen as they are readily removable by a variety of methods, whereas the deprotection of benzylamines cannot always be easily realised. ${ }^{93}$ Hopefully, reaction conditions could be found for clean deprotection of $\mathbf{2 . 7 0}$, so that minimal purification of $\mathbf{1 . 5 0}$ would be required.

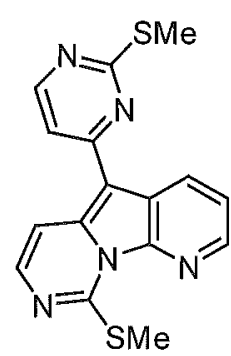

2.1

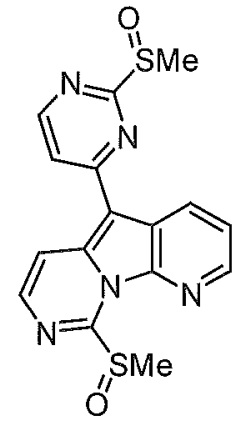

2.69

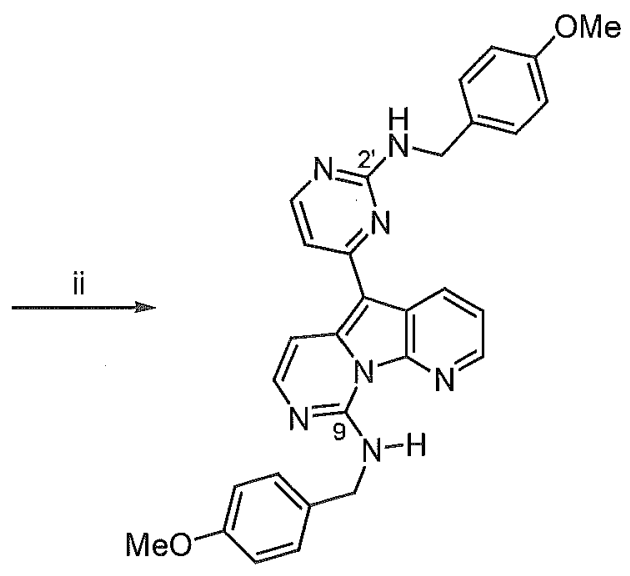

2.70

Scheme 2.12 Reagents and yields: (i) $m$-CPBA (1.9 equiv) $/ \mathrm{CHCl}_{3} /-15^{\circ} \mathrm{C}$; (ii) $p$-methoxybenzylamine (26 equiv) $/ 90{ }^{\circ} \mathrm{C}$ (62\% from 2.1 ).

Thus, the crude oxidised mixture was taken, without further purification or characterisation, and heated in neat $p$-methoxybenzylamine. After $14 \mathrm{~h}$ at $90^{\circ} \mathrm{C}$, TLC analysis showed just one yellow spot. Careful flash chromatography allowed the isolation of the desired product $\mathbf{2 . 7 0}$ in $62 \%$ yield over the two steps (Scheme 2.12). The presence of two PMB groups was clearly seen in the ${ }^{1} \mathrm{H}$ NMR spectrum. In addition to the aromatic and methoxy signals, two doublets were observed at $\delta 4.69$ and 4.89 for each of the benzyl methylene groups. The first doublet was coupled to a broad signal at $\delta 5.52$, assigned to the $2^{\prime}-\mathrm{NH}$ proton. The second doublet was 
coupled to a slightly broadened triplet at $\delta 10.36$, which was assigned to the $9-\mathrm{NH}$ proton. The downfield chemical shift of this signal can be explained by hydrogen bonding to the pyridine nitrogen (N1), which has been seen in the natural product variolin $\mathrm{B}^{32 \mathrm{a}}$ Interestingly, when the NMR sample of 2.70 was shaken with $\mathrm{D}_{2} \mathrm{O}$, this proton did not exchange. This provides further confirmation of a strong hydrogen bond between these two atoms. No hydrogen bond acceptor is available for the $2^{\prime}-\mathrm{NH}$ proton, hence its appearance much further upfield. The signals from the pyrimidine protons all move upfield, as expected, upon introduction of the electron-donating amines.

The introduction of both amine functionalities in one step was a pleasing result. In addition to taking the synthesis of deoxyvariolin B (1.50) one step closer to completion, this methodology should allow incorporation of an almost limitless range of amines (and presumably other nucleophiles), the products of which can be tested in a range of assays for potency and other desirable pharmacological properties.

\subsubsection{Removal of the $p$-Methoxybenzyl Protecting Groups}

The removal of both $\mathrm{N}$-protecting groups would result in a concise synthesis of deoxyvariolin B. It would also validate this methodology for application to the natural product variolin $\mathrm{B}(\mathbf{1 . 1 7})$. $p$-Methoxybenzyl groups are often removed under oxidative conditions, occasionally with DDQ (2,3-dichloro-5,6-dicyano-1,4-benzoquinone), ${ }^{94}$ but more commonly with CAN (ceric ammonium nitrate). ${ }^{93}$ Attempted deprotection of 2.70 with DDQ produced a number of minor products, but mainly starting material was retained. The use of CAN led to a complex mixture in which no variolin-type compound could be detected.

Another simple method for deprotection of PMB groups, although not so commonly used, involves standing the protected amine in neat TFA at room temperature. ${ }^{95}$ This was deemed appropriate for the variolins, as they are reported to be stable to acid and, indeed, are most easily handled as their trifluoroacetate salts. ${ }^{3230}$ Dissolving 2.70 in TFA immediately produced a blood-red solution. After $24 \mathrm{~h}$, TLC analysis indicated that a significant portion of starting material remained, so the reaction was heated at $60^{\circ} \mathrm{C}$. After $3 \mathrm{~h}$ at this temperature, one major 
product was observed, which was of greater polarity than 2.70 . From the ${ }^{1} \mathrm{H}$ NMR spectrum of the crude reaction mixture, it appeared that only one PMB group had been removed.

When the stronger acid triflic acid was used, a new product of even greater polarity was produced after just $4 \mathrm{~h}$ at room temperature (Scheme 2.13). The crude ${ }^{1} \mathrm{H}$ NMR spectrum (in DMSO- $\mathrm{d}_{6}$ ) contained just seven aromatic signals, consistent with what would be expected from deoxyvariolin B (1.50). Purification of the product from the PMB byproducts proved difficult, requiring the use of reversed phase chromatography. A yellow band eluted (when $70 \%$ $\mathrm{MeOH} /$ water $+0.1 \%$ TFA was used as the eluant) to give an acidic product. This was neutralised with solid potassium carbonate and extracted with boiling $\mathrm{CH}_{2} \mathrm{Cl}_{2}$ (in which it is only sparingly soluble), to afford deoxyvariolin $\mathrm{B}$ in an acceptable yield of $56 \%$.

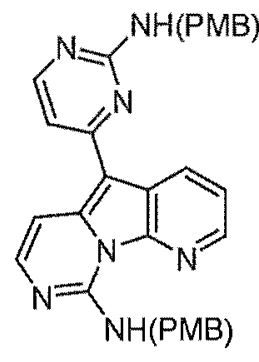

2.70

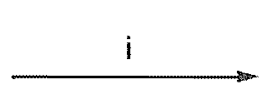

1.50

deoxyvariolin $\mathbf{B}$

Scheme 2.13 Reagents and yields: (i) triflic acid/rt (56\%).

The ${ }^{1} \mathrm{H}$ NMR spectrum, shown in Figure 2.31, contains seven aromatic signals and displays no peaks below $6.5 \mathrm{ppm}$, consistent with the removal of both $N$-protecting groups. The usual 2D NMR experiments were used to assign the ${ }^{1} \mathrm{H}$ and ${ }^{13} \mathrm{C}$ signals. As with 2.1, the pyrimidine signals were assigned with the aid of an NOE experiment. Thus, H5' $(\delta 7.05)$ exhibited NOEs with three other protons: $\mathrm{H} 6{ }^{\prime}$ (its coupling partner, $\left.\delta 8.22\right), \mathrm{H} 4(\mathrm{dd}, \delta 8.90)$ and $\mathrm{H} 6(\delta 7.67)$. The signals for the $\mathrm{NH}_{2}$ protons are similar to those seen for variolin $\mathrm{B}$. The $2-\mathrm{NH}_{2}$ protons give rise to a sharp singlet at $\delta 6.51$, integrating for two protons. Two broad signals at $\delta 8.5$ and 9.4 are seen for the 9- $\mathrm{NH}_{2}$ protons. As with variolin $\mathrm{B}(1.17)$, this is explained by restricted rotation around the $\mathrm{C} 9-\mathrm{N}$ bond which is, itself, a consequence of hydrogen bonding between one of the 
9- $\mathrm{NH}_{2}$ protons and $\mathrm{N} 1$ of the pyridine ring. The hydrogen bonded proton is not as deshielded as that of variolin B, whose signal appears at $\delta 9.8$. This difference is best explained by the absence of the hydroxyl group on the pyridine ring of variolin B. Its electron-donating effect would make N1 of variolin B a stronger hydrogen bond donor than that of deoxyvariolin B, with the consequence that the $9-\mathrm{NH}$ proton of variolin $\mathrm{B}$ is more deshielded.

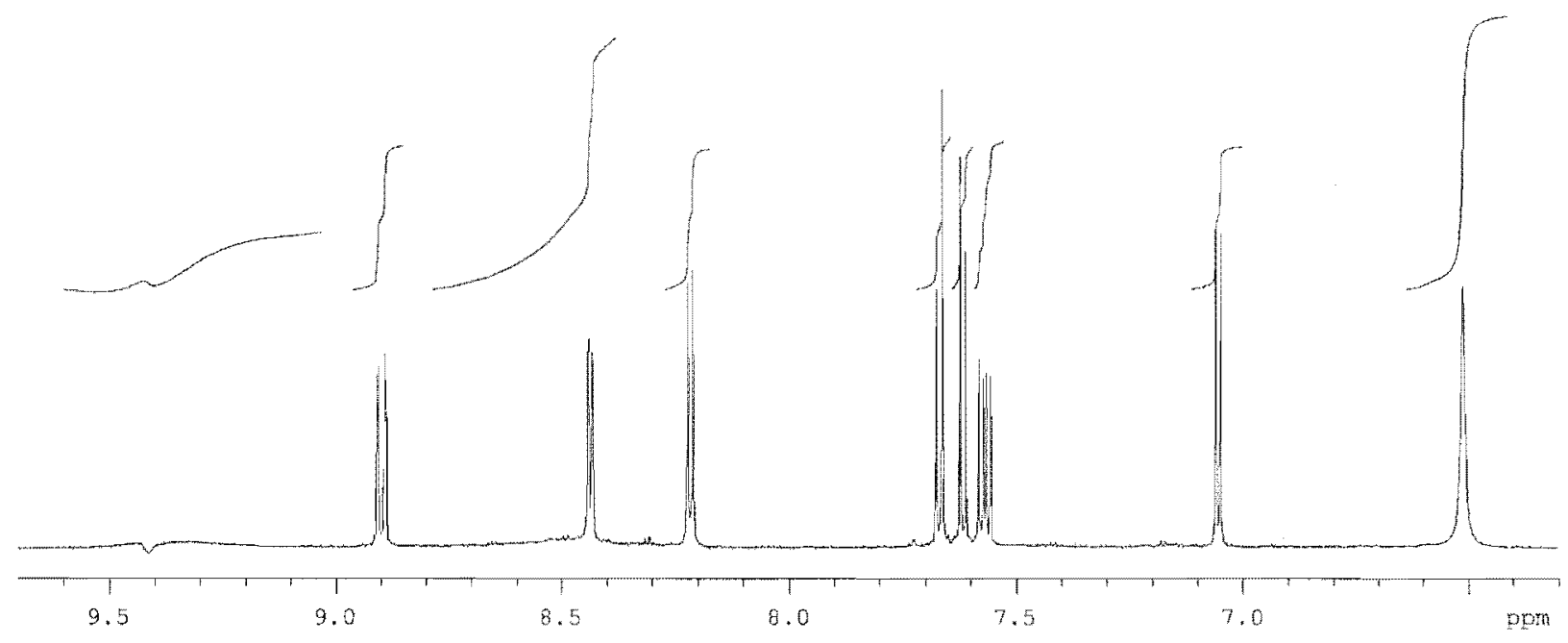

Figure 2.31 $500 \mathrm{MHz}{ }^{1} \mathrm{H}$ NMR spectrum of deoxyvariolin B (1.50) in DMSO-d $\mathrm{d}_{6}$. The phasing artefact at $\delta 9.4$ is of unknown origin, and was also present in the spectrum of the solvent alone.

Further comparisons of the ${ }^{1} \mathrm{H}$ and ${ }^{13} \mathrm{C}$ NMR data can be drawn between deoxyvariolin $\mathrm{B}$ and the natural product variolin B from Table 2.2. The major differences lie in positions 3 and 4 a (due to the $+\mathrm{R}$ effect of the hydroxyl group of variolin $\mathrm{B}$ ), and $\mathrm{C} 4$ (due to the $-I$ effect of the hydroxyl group). Also noteworthy is $\mathrm{H} 6$ of deoxyvariolin B, whose signal appears almost $0.5 \mathrm{ppm}$ downfield of that in the natural product. This is presumably due to the anisotropic deshielding effect of the pendant pyrimidine ring (see discussion on p 55). $\mathrm{H} 4$ also gives rise to an unusually downfield signal, presumably for the same reason. 


\begin{tabular}{|c|c|c|c|c|c|c|}
\hline \multirow[b]{2}{*}{ position } & \multicolumn{3}{|c|}{${ }^{1} \mathrm{H}(\mathrm{ppm})$} & \multicolumn{3}{|c|}{${ }^{13} \mathrm{C}(\mathrm{ppm})$} \\
\hline & $1.50^{\mathrm{a}}$ & $1.17^{b}$ & $1.50^{\mathrm{e}}$ & $1.50^{\mathrm{d}}$ & 1.17 & $1.50^{\circ}$ \\
\hline 2 & 8.44 & 8.14 & 8.51 & 140.3 & 143.2 & 143.8 \\
\hline 3 & 7.57 & 6.78 & 7.64 & 121.0 & 107.6 & 120.7 \\
\hline 4 & 8.90 & & 8.99 & 129.4 & 159.9 & 129.1 \\
\hline $4 a$ & & & & 121.7 & 111.2 & 121.6 \\
\hline 5 & & & & 99.6 & 99.6 & 99.5 \\
\hline $5 \mathrm{a}$ & & & & 138.3 & 137.1 & 142.8 \\
\hline 6 & 7.67 & 7.19 & 7.70 & 101.9 & 100.4 & 101.7 \\
\hline 7 & 7.62 & 7.61 & 7.76 & 144.0 & 144.6 & 140.1 \\
\hline 9 & & & & 149.9 & 150.3 & $163.5^{*}$ \\
\hline $10 \mathrm{a}$ & & & & 143.0 & 145.0 & 149.7 \\
\hline $2^{\prime}$ & & & & 163.6 & 158.4 & $161.4^{*}$ \\
\hline $4^{\prime}$ & & & & 161.6 & 161.5 & 138.2 \\
\hline $5^{\prime}$ & 7.05 & 7.11 & 7.12 & 107.1 & 106.1 & 106.8 \\
\hline $6^{\prime}$ & 8.22 & 8.25 & 8.29 & 158.2 & 160.1 & 158.0 \\
\hline 9- $\mathrm{NH}_{2}$ & 8.5 & 8.5 & & & & \\
\hline 9- $\mathrm{NH}_{2}$ & 9.4 & 9.8 & & & & \\
\hline $2^{\prime}-\mathrm{NH}_{2}$ & 6.51 & 6.95 & $6.63(1 \mathrm{H})$ & & & \\
\hline $4-\mathrm{OH}$ & & 16.0 & & & & \\
\hline
\end{tabular}

Table 2.2 ${ }^{1} \mathrm{H}$ and ${ }^{13} \mathrm{C}$ NMR chemical shifts for deoxyvariolin $\mathrm{B}(1.50)$ and variolin $\mathrm{B}$ $(1.17) .^{32 \mathrm{a}}$

${ }^{a}$ Recorded in DMSO- $\mathrm{d}_{6}$ at $500 \mathrm{MHz}$. ${ }^{\mathrm{b}}$ These values have been adjusted by $-0.12 \mathrm{ppm}$ from those in the original paper, so that the solvent reference peak $(\delta 2.62)$ corresponds to the present system $(\delta 2.50 \mathrm{ppm})$. c These values reported by Álvarez, Fernández and Joule. ${ }^{38}$ (No value was provided for the solvent reference peak.) ${ }^{d}$ Recorded in DMSO- $\mathrm{d}_{6}$ at $75 \mathrm{MHz}$.

The NMR data for deoxyvariolin B (1.50) reported by Álvarez, Fernández and Joule has also been included in the Table. The ${ }^{1} \mathrm{H}$ chemical shifts of this thesis are consistently lower than those of Álvarez et al, probably due to differences in solvent referencing. However, it is likely that $\mathrm{H} 6$ and $\mathrm{H} 7$ have been confused by these workers - an easy trap to fall into if assignments are based solely on chemical shift arguments. Their ${ }^{13} \mathrm{C}$ assignments also appear to be rather muddled. Chemical shifts are likely to differ, somewhat, with small differences in $\mathrm{pH}$ and 
possibly the amount of water present in the solvent. However, their chemical shifts compare very well to those obtained in this thesis (all are within $0.3 \mathrm{ppm}$ ); therefore, it is their assignments which are at fault. It should be emphasised that all new comounds in this thesis were characterised by 2D NMR techniques (COSY, HSQC and HMBC or CIGAR experiments). If any uncertainties regarding signal assignments existed, these are made clear in the Experimental section of this thesis (Chapter Six). 


\subsection{Summary}

Using the strategy shown in Figure 2.1, a convenient and direct synthesis of the pyrido[3; $\left.2^{*}: 4,5\right]$ pyrrolo[1,2-c]pyrimidine ring system, found in all of the variolins, has been realised. Conditions were found to optimise the formation of the variolin core structure 2.1, which was produced in just three steps from commercially available materials. In addition, the route was extended to provide a six-step synthesis of deoxyvariolin B (1.50), which is summarised in Scheme 2.14. The overall yield of this sequence was $5.2 \%$. This compares favourably to the synthesis reported by Alvarez et al., which proceeded in $2.2 \%$ yield over 14 steps. $^{38}$

This work employs a strategy which should be readily adapted to a total synthesis of the natural product variolin B (1.17). It has also been highlighted that the method is particularly suited to the production of analogues which vary in their substituents at $\mathrm{C} 9$ and C2'. This will allow structure-activity-relationship (SAR) studies to be undertaken, which may result in new leads for potential drug-development. 


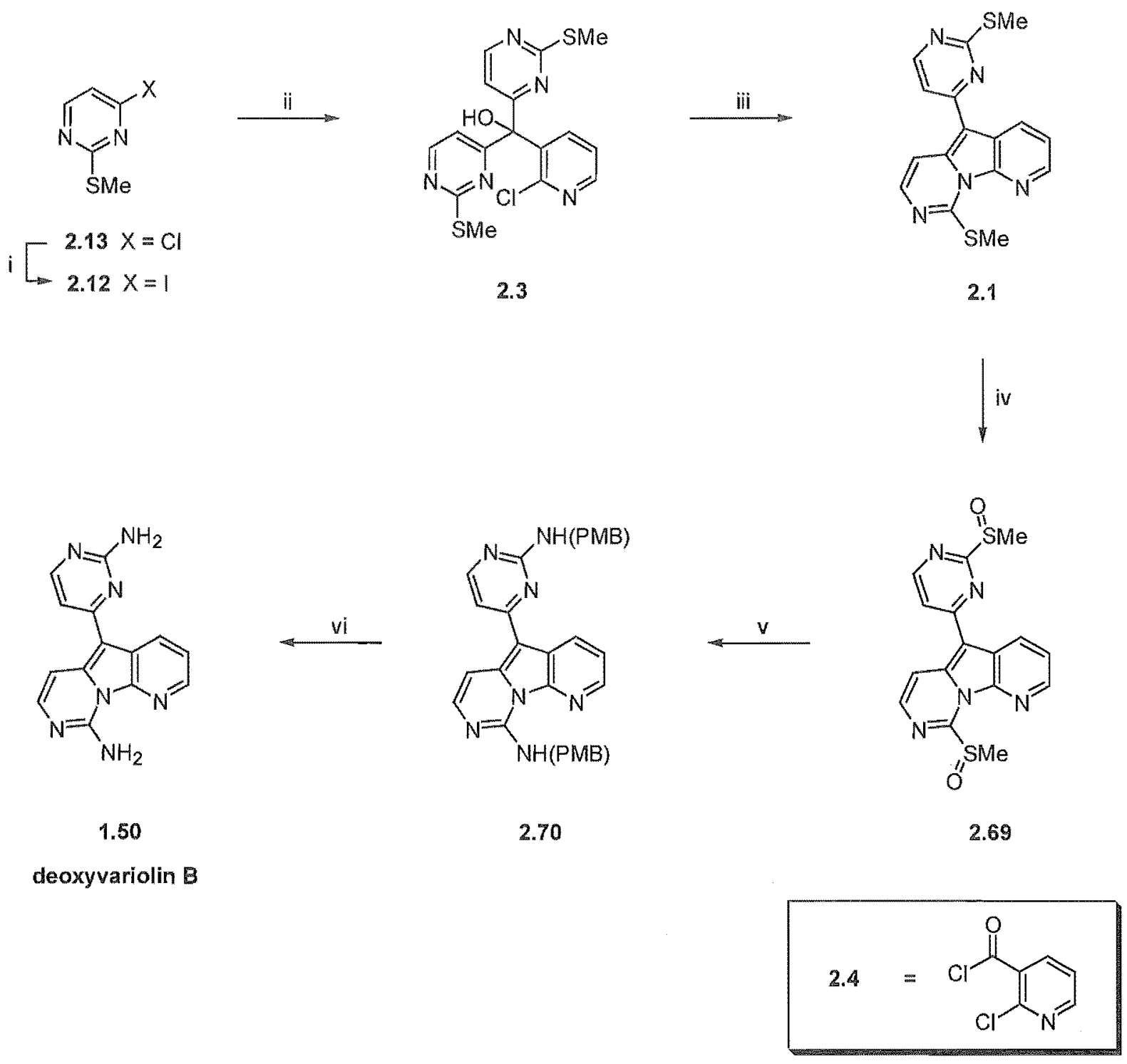

Scheme 2.14 Reagents and yields: (i) $55 \%$ aq HI/rt (79\%); (ii) $n$-BuLi (1 equiv)/THF/ $-95{ }^{\circ} \mathrm{C}, 30 \mathrm{~min}$ then $2.4\left(0.33\right.$ equiv)/THF $/-95^{\circ} \mathrm{C}, 3.5 \mathrm{~h}(56 \%)$; (iii) TFA ( 4 equiv)/TES ( 8 equiv) $/ 75^{\circ} \mathrm{C}$ (34\%); (iv) $m$-CPBA (1.9 equiv) $/ \mathrm{CHCl}_{3} /-15{ }^{\circ} \mathrm{C}$; (v) $p$-methoxybenzylamine (26 equiv) $/ 90$ ${ }^{\circ} \mathrm{C}(62 \%$ from 2.1$)$; (vi) triflic acid/rt $(56 \%)$. 


\section{Chapter Three}

\section{The Total Synthesis of}

Variolin B 


\subsection{Synthesis of Triarylmethanol 1.65}

As has been discussed in Chapter Two, a direct route to the pyrido[3', $\left.2^{\prime}: 4,5\right]$ pyrrolo[1,2c]pyrimidine ring system found in the variolin natural products has been developed, employing the tandem deoxygenation/cyclisation of a triarylmethanol as the key step. Having shown that this methodology was applicable to a synthesis of deoxyvariolin B, our attention turned to the application of this methodology to the synthesis of variolin B (1.17).

The retrosynthetic analysis for variolin B is displayed in Figure 3.1. It must be noted that this has been modified slightly from the original plan discussed in Chapter One due to the results obtained for the deoxy-system (Chapter Two). The retrosynthesis differs from that of deoxyvariolin $\mathrm{B}$ by the requirement of the hydroxyl group in the natural product. In our retrosynthetic plan, this group is protected as the methyl ether throughout most of the sequence. At some stage, its deprotection would be required. It was intended that this would be carried out after the variolin core had been constructed, however, the exact order of steps may depend upon the reactivity, stability and ease of handling of the intermediates in the sequence.

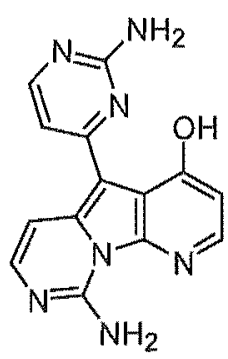

1.17 variolin $B$
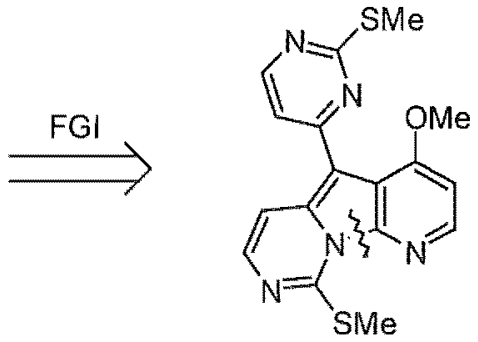

1.63<smiles>COc1ccnc(Cl)c1C(O)(c1ccnc(SC)n1)c1ccnc(SC)n1</smiles>

1.65<smiles>C=CCCC</smiles><smiles>COc1ccnc(Cl)c1C(=O)Cl</smiles>

Tigure 3.1 Retrosynthetic analysis of variolin B (1.17). 


\subsubsection{Synthesis of the Acid Chloride 1.67}

Adaptation of the methodology developed in Chapter Two to variolin B (1.17) requires access to 2-chloro-4-methoxynicotinoyl chloride (1.67). While neither this compound, nor the parent carboxylic acid, have been reported in the literature, the bromo analogues have. ${ }^{96}$ Kasum and Prager generated 2-bromo-4-methoxynicotinic acid (3.1) via an efficient four step sequence shown in Scheme 3.1. This compound was required as an intermediate in the proposed synthesis of the alkaloid perloline. First, condensation of ethyl cyanoacetate (3.2) with trimethyl orthoacetate (3.3) gave ethyl 2-cyano-3-methoxybut-2-enoate (3.4). This product contains the methoxy group that would later be found at position 4 of the nicotinic acid. Heating with dimethylformamide dimethyl acetal gave enamine 3.5 , which could be readily cyclised to bromopyridine 3.6 by treatment with anhydrous $\mathrm{HBr}$ in acetic acid. The ester was hydrolysed with aqueous sodium hydroxide solution to give the carboxylic acid 3.1 .

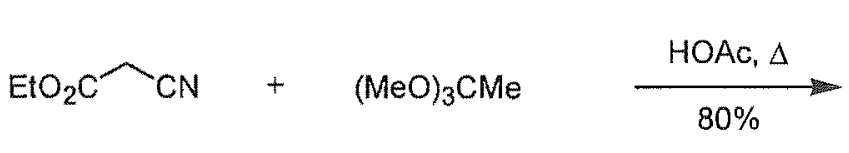

3.2<smiles>CCOC(=O)/C(C#N)=C(\C)OC</smiles>

3.4

$$
85 \% \mid \begin{aligned}
& \mathrm{Me}_{2} \mathrm{NCH}(\mathrm{OMe})_{2} \\
& 140^{\circ} \mathrm{C}
\end{aligned}
$$<smiles>[R]Oc1ccnc(Br)c1C(=O)O[Na]</smiles><smiles>CCOC(=O)CCOC(C)=O</smiles><smiles>CCOC(=O)C(C#N)=C(C=CN(C)C)OC</smiles>

3.5

$$
\underset{80 \%}{\operatorname{aq~NaOH}, \text { reflux }}\left[\begin{array}{c}
3.6 \mathrm{R}=\mathrm{Et} \\
3.1 \mathrm{R}=\mathrm{H}
\end{array}\right.
$$


If anhydrous $\mathrm{HCl}$ could be substituted for $\mathrm{HBr}$, the chloro analogue of $\mathbf{3 . 6}$ should be produced. Indeed, bubbling dry $\mathrm{HCl}$ gas through a solution of 3.5 (produced by Kasum and Prager's method) in acetic acid produced the desired chloropyridine 3.7 (Scheme 3.2). This was partially purified by vaccum distillation, with the remaining impurities being conveniently removed in the following step. A minor product of this reaction was 2-chloro-4-methoxypyridine (3.8). This must be formed by ester hydrolysis and subsequent decarboxylation, although it is not known if this occurs before formation of the pyridine ring, or after. Hydrolysis of ester $\mathbf{3 . 7}$ provided the carboxylic acid 3.9 in $77 \%$ yield for the two steps. No purification was necessary, other than repeated washing of an aqueous alkaline solution of $\mathbf{3 . 9}$ with an organic solvent. Refluxing 3.9 in thionyl chloride with catalytic DMF did not cleanly produce the desired acid chloride 1.67, which is in contrast to the deoxy-equivalent 2.4 . However, 1.67 was formed in $97 \%$ yield when oxalyl chloride was used in place of thionyl chloride.

3.2

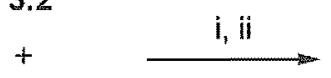

3.3<smiles>CCOC(=O)C(C#N)=C(C=CN(C)C)OC</smiles>

3.5

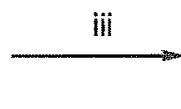

iv

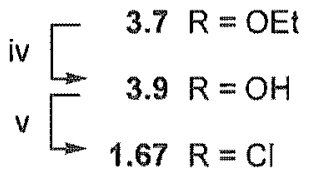<smiles>[R]C(=O)c1c(OC)ccnc1Cl</smiles>

3.8

Scheme 3.2 Reagents and yields: (i) $\mathrm{HOAc} / \triangle$ (70\%); (ii) $\mathrm{Me}_{2} \mathrm{NCH}(\mathrm{OMe})_{2} / 140{ }^{\circ} \mathrm{C}(79 \%)$; (iii) dry $\mathrm{HCl} / \mathrm{HOAc} / 65-70{ }^{\circ} \mathrm{C}$; (iv) $1.5 \mathrm{M}$ aq $\mathrm{NaOH} /$ reflux $(77 \%$ from 3.5$)$; (v) $(\mathrm{COCl})_{2} / \mathrm{cat}$. $\mathrm{DMF} / \mathrm{CH}_{2} \mathrm{Cl}_{2} / \mathrm{rt}(97 \%)$.

\subsubsection{Addition of the Pyrimidine to Acid Chloride 1.67}

\subsubsection{Use of the Lithiated Pyrimidine 1.66}

With a straightforward route to the acid chloride, the double addition of lithio-species 1.66 was attempted using the conditions previously established for the synthesis of triarylmethanol $\mathbf{2 . 3}$. Disappointingly, after $4.5 \mathrm{~h}$ at $-95{ }^{\circ} \mathrm{C}$, the triarylmethanol 1.65 was isolated in less than $15 \%$ 
yield (Scheme 3.3). The side-products of the lithiation step 2.20-2.22 were, once again, all observed in the present reaction. In fact, the deiodinated pyrimidine $\mathbf{2 . 2 0}$ was the major component of the mixture. However, two other products were isolated from the reaction - the biaryl ketone $\mathbf{3 . 1 0}$ and biaryl alcohol 3.11. These products are analogous to ketone 2.11 and alcohol 2.23, which were formed as side-products during the synthesis of triarylmethanol $\mathbf{2 . 3}$ (Chapter Two). Both $\mathbf{3 . 1 0}$ and $\mathbf{3 . 1 1}$ were present in greater quantities than the triarylmethanol 1.65, with the ketone 3.10 being formed in $\sim 25 \%$ yield and the alcohol 3.11 in $15 \%$ yield.

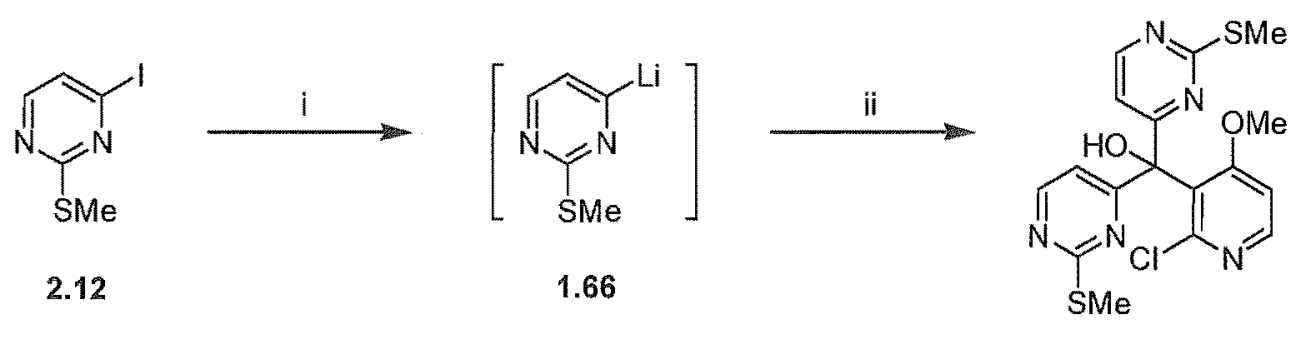

$$
1.65
$$

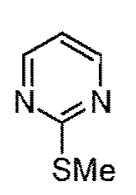

2.20<smiles>CSc1nccc(-c2ccnc(SC)n2)n1</smiles>

2.21<smiles>CSc1nccc(-c2cc(-c3ccnc(SC)n3)nc(SC)n2)n1</smiles>

2.22<smiles>COc1ccnc(Cl)c1C(=O)c1ccnc(SC)n1</smiles>

3.10<smiles>COc1ccnc(Cl)c1C(O)c1ccnc(SC)n1</smiles>

3.11

Scheme 3.3 Reagents and yields: (i) $n$-BuLi (1 equiv)/THF/ $-95{ }^{\circ} \mathrm{C}, 30 \mathrm{~min}$; (ii) 1.67 (0.33 equiv) $/ \mathrm{THF} /-95^{\circ} \mathrm{C}, 4.5 \mathrm{~h}(<15 \%)$.

When the reaction was quenched at $-95^{\circ} \mathrm{C}$ with deuterated methanol $\left(\mathrm{CH}_{3} \mathrm{OD}\right)$, both 4-deuterio2-(methylsulfanyl)pyrimidine (2.27) and the methyl ester 3.12 were observed. As discussed in 
Chapter Two, this clearly indicates that both the lithio-species 1.66 and acid chloride 1.67 were present in the reaction mixture at the time of quenching. Evidently, longer reaction times would be required to improve the yield of the triarylmethanol 1.65 . Clearly, the presence of large amounts of the ketone 3.10 also leads to this conclusion.<smiles>[2H]c1cc([2H])nc([2H])n1</smiles>

2.27<smiles>COC(=O)c1c(OC)ccnc1Cl</smiles>

3.12

Conducting such a reaction for extended periods requires regular top-ups of the cooling bath $\left(\mathrm{MeOH} /\right.$ liquid $\mathrm{N}_{2}$ ) with liquid nitrogen to ensure that the temperature does not rise above $-95^{\circ} \mathrm{C}$. An all-night vigil allowed the reaction to be stirred at $-95^{\circ} \mathrm{C}$ for at least $20 \mathrm{~h}$, followed by gradual warming to room temperature." Every precaution was taken to ensure optimum results. These included the use of standard inert atmosphere techniques; freshly purified solvents and reagents; the use of the reactor described in Chapter Two (Figure 2.8, p 41); slow, smooth addition of reagents (both $n$-BuLi and acid chloride 1.67); and careful monitoring of the cooling bath temperature, such that it never rose above $-93^{\circ} \mathrm{C}$. Much to our consternation and disgust, the ${ }^{1} \mathrm{H}$ NMR spectrum of the crude material showed only trace amounts of the desired triarylmethanol 1.65. The major product was, once again, the deiodinated pyrimidine 2.20. This was followed closely by the product of mono-addition, ketone 3.10. A small, but significant, amount of the methyl ester $\mathbf{3 . 1 2}$ was also present as the reaction was quenched (after warming somewhat) with methanol. As usual, small amounts of the biaryl alcohol 3.11, bipyrimidine 2.21 and the trimeric product 2.22 were also present in the mixture.

The conclusion from this egregious result is that the second addition of the lithio-species $\mathbf{1 . 6 6}$ to the ketone 3.10 is extremely slow at $-95^{\circ} \mathrm{C}$. This is in contrast to the double addition of 1.66 in the deoxy-series, where good yields of triarylmethanol 2.3 were obtained after $3.5 \mathrm{~h}$ at $-95^{\circ} \mathrm{C}$. The presence of the methoxy group on the pyridine ring must greatly hinder attack at the

\footnotetext{
"Julian Browne is gratefully acknowledged for taking part of the "night watch".
} 
carbonyl group of both the acid chloride 1.67 and the biaryl ketone 3.10. A crystal structure of the ketone was obtained and is shown in Figure 3.2. In this structure, the two aromatic rings lie almost orthogonal to each other. This causes the methoxy group and the chlorine of the pyridine ring to block both faces of the carbonyl group, making attack by a nucleophile-particularly a large one such as the pyrimidinyllithium 1.66 extremely difficult.

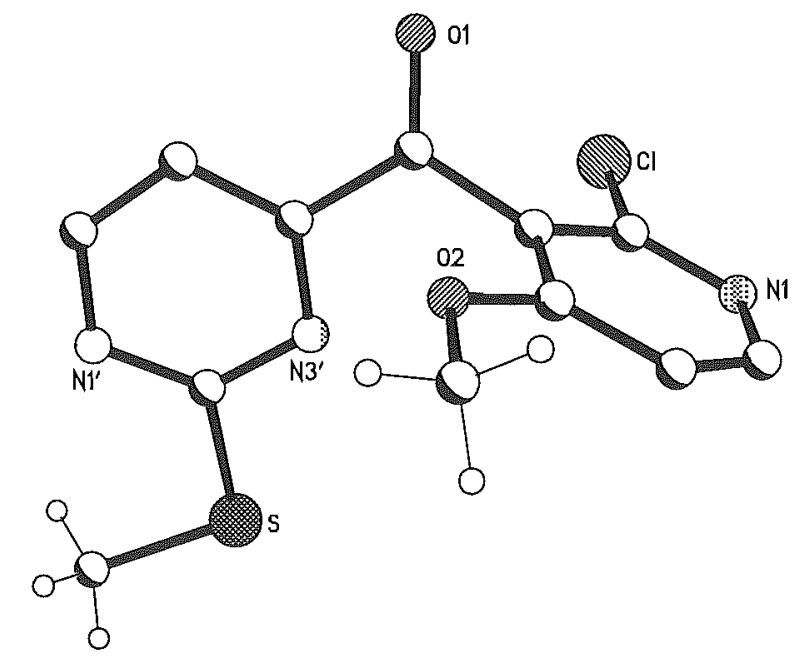

Figure 3.2 X-ray crystal structure of 3.10. Aromatic hydrogens have been omitted for clarity.

The sensitivity of these reactions to temperature should be highlighted. Product ratios differed, somewhat, from one reaction to another. It seems that slight (unintentional) increases in temperature resulted in greater amounts of the triarylmethanol being formed. But this would be accompanied by increased proportions of side products such as the biarylmethanol 3.11. In no instance was the triarylmethanol ever isolated in greater than $15 \%$ yield.

\subsubsection{DMPU as Cosolvent}

To improve this reaction, it was necessary to both increase the nucleophilicity of the heteroaryllithium species and decrease its basicity, so as to favour attack at the carbonyl group over unwanted side-reactions. Additives such as hexamethylphosphoramide (HMPA) ${ }^{97}$ and, more recently, $N, N^{\prime}$-propyleneurea (DMPU) ${ }^{98}$ have been widely used to increase the 
nucleophilicity of organometallic reagents. Organolithium and magnesium reagents commonly form oligomeric structures in non-polar or weakly polar solvents (hydrocarbons, diethyl ether). In more strongly coordinating solvents such as $\mathrm{THF}$, these aggregates are broken down to some extent, which has the effect of increasing the reactivity of the organometallic reagent. ${ }^{99}$ The addition of dipolar cosolvents such as HMPA or DMPU can cause further deaggregation to give monomeric species. HMPA has also been shown to coordinate to the lithum cations of a number of organolithium species, ${ }^{100}$ leaving a "naked" anion with enhanced nucleophilicity.

It was thought that the use of a polar cosolvent may enhance the nucleophilicity of 1.66. For these studies, $t$-BuLi (two equivalents) was used for lithium-iodine exchange to avoid the generation of the potentially reactive $n$-BuI, which would be formed if $n$-BuLi was used. The use of two equivalents of $t$-BuLi destroys any alkyl iodide produced, as $t$-BuI immediately eliminates $\mathrm{HI}$ in the presence of the second equivalent of base to produce 2 -methylpropene and isobutane (Figure 3.3), which normally do not interfere with subsequent reactions. ${ }^{101}$ DMPU was chosen as the cosolvent, primarily due to its lower toxicity when compared to the highly mutagenic HMPA.

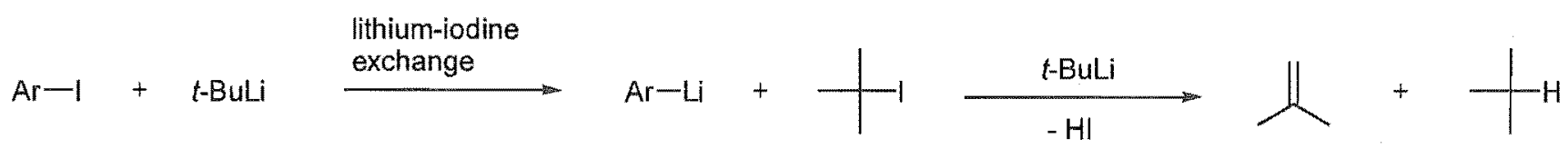

Figure 3.3 Lithium-iodine exchange using $t$-BuLi as metallating agent.

However, lithiation of iodopyrimidine 2.12 in THF using two equivalents of $t$-BuLi, followed by the addition of DMPU (10\% volume relative to THF) and then acid chloride 1.67 did not improve the outcome of the reaction. In fact, the only recognisable product in the mixture was the familiar pyrimidine 2.20. The presence of various signals in the ${ }^{1} \mathrm{H}$ NMR spectrum at $\delta 4.5-$ 5.5 may indicate nucleophilic addition to the $\pi$-deficient heterocyclic rings to give dihydropyridines and/or -pyrimidines. As a consequence, the use of polar cosolvents was not explored any further. 


\subsubsection{Organocerium Reagents}

Organocerium reagents have been shown to be considerably less basic than the corresponding organolithium or Grignard reagents, while being of comparable or greater nucleophilicity. ${ }^{102}$ These reagents have been especially useful for nucleophilic addition to enolisable carbonyl groups, whereas the more basic organolithium and Grignard reagents often give low yields due to competitive enolisation.

The organocerium reagents are generally derived by transmetallation of either organolithium species at low temperature $\left(-78^{\circ} \mathrm{C}\right)$ or Grignard reagents at $0{ }^{\circ} \mathrm{C}$. If the cerium equivalent of 1.66 could be generated, it may provide an effective means of avoiding unwanted side-products and increasing the yield of the triarylmethanol 1.65. It may also allow higher reaction temperatures to be used if it is less prone to protonation than 1.66. The lithio-species was generated at $-95^{\circ} \mathrm{C}$ in the usual manner and a suspension of dry $\mathrm{CeCl}_{3}$ in $\mathrm{THF},{ }^{102 a}$ which had also been cooled to $-95^{\circ} \mathrm{C}$, was transferred to the yellow-brown solution. A colour-change to red-brown was observed and the solution was allowed to stir for $0.5 \mathrm{~h}$ at $-80^{\circ} \mathrm{C}$, after which time, the acid chloride $\mathbf{1 . 6 7}$ was introduced. However, prolonged stirring at this temperature gave no triarylmethanol 1.65, nor even the ketone 3.10. Despite quenching the reaction with methanol, the methyl ester was not isolated either. In fact, the only pyridine-containing product appeared to be the starting material, acid chloride 1.67 , which must have survived both methanolysis and aqueous workup The major product was, once again, the pyrimidine $\mathbf{2 . 2 0}$, accompanied by small amounts of 2.21 and 2.22 .

When the reaction was repeated, but using reverse addition (i.e. the cooled lithio-species 1.66 was added to $\mathrm{CeCl}_{3}$ via a cannula), the results were the same except that much more of the bipyrimidine 2.21 was present (and correspondingly less 2.20 ). The predominance of 2.21 in this case is probably due to significant warming of the unstable lithio-species during transfer, even though it had been cooled to below $-100^{\circ} \mathrm{C}$ immediately before transfer. Even so, some active aryl-metal species (either lithium or cerium) was still present after $3.5 \mathrm{~h}$ at $-78^{\circ} \mathrm{C}$, as evidenced by quenching with $\mathrm{CH}_{3} \mathrm{OD}$. However, it should be reiterated that the only pyridinecontaining species observed in any significant amount was the starting material 1.67. Neither the biaryl ketone $\mathbf{3 . 1 0}$ nor triarylmethanol 1.65 were produced. 
When the ketone 3.10 was used as the electrophile in place of the acid chloride, starting material was again recovered, along with the usual pyrimidine byproducts. It has been observed that amine and alkoxide ligands greatly reduce the reactivity of organocerium species, presumably by coordination to the cerium. ${ }^{102 \mathrm{~b}}$ It is possible that the abundance of heterocyclic nitrogens in the present system may contribute to the pyrimidinylcerium species' pronounced unreactivity. However, it is unclear if the cerium reagent had even formed. In any case, it was evident that this line of work offered no improvements to the synthesis of triarylmethanol 1.65.

\subsubsection{Pyrimidinyl Grignard Reagents}

Around this time, Quéguiner and coworkers reported their investigations into the use of the pyrimidinyl Grignard reagent 3.13. ${ }^{56}$ This species is formed from the iodide 2.12 by iodinemagnesium exchange with $\mathrm{i}-\mathrm{PrMgCl}$ and is reported to be stable at $0^{\circ} \mathrm{C}$. Reaction with a limited range of electrophiles gave moderate to good yields of substituted pyrimidines $\mathbf{3 . 1 4}$ (Scheme 3.4).

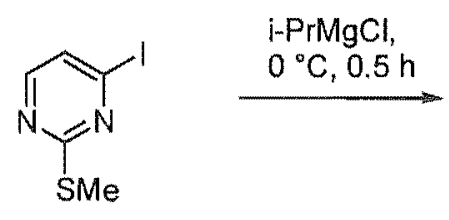

2.12

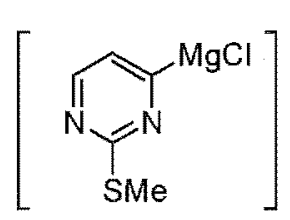

3.13

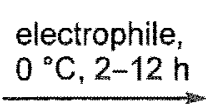<smiles>CSc1nccc(F)n1</smiles>

3.14

\begin{tabular}{lll}
\hline Electrophile & E & Yield (\%) \\
\hline PhCHO & PhCH(OH) & 57 \\
$\mathrm{C}_{5} \mathrm{H}_{11} \mathrm{CHO}$ & $\mathrm{C}_{5} \mathrm{H}_{11} \mathrm{CH}(\mathrm{OH})$ & 55 \\
$\mathrm{NC}-\mathrm{COOEt}$ & $-\mathrm{COOEt}$ & 35 \\
DMF & $-\mathrm{CHO}$ & 34 \\
\hline
\end{tabular}

Scheme 3.4 
It was hoped that 3.13 would be reactive enough to add to acid chloride 1.67 at $0{ }^{\circ} \mathrm{C}$. However, after stirring the two reagents at $0{ }^{\circ} \mathrm{C}$ for $3.5 \mathrm{~h}$ and quenching with $\mathrm{CH}_{3} \mathrm{OD}$, the major product was the pyrimidine 2.20, along with some of the deuterated equivalent 2.27. A significant amount of the bipyrimidine $\mathbf{2 . 2 1}$ had also formed at this temperature. Only traces of the triarylmethanol 1.65 were observed and the ketone $\mathbf{3 . 1 0}$ was present in even lower amounts. Surprisingly, the acid choride was not recovered, either in that form or as its methyl ester $\mathbf{3 . 1 2 .}$

When the reaction was repeated, but at $-78{ }^{\circ} \mathrm{C}$ (after formation of the Grignard species at $0{ }^{\circ} \mathrm{C}$ ), the deuterated pyrimidine 2.27 was, by far, the major product. Again, almost none of the methyl ester was produced, despite quenching with $\mathrm{CH}_{3} \mathrm{OD}$ at $-78{ }^{\circ} \mathrm{C}$ and then allowing to warm to room temperature. It appears that the acid chloride is destroyed by the Grignard reagent. In both reactions, it was noted that upon addition of the acid chloride, a sudden colour change from brown to bright red occurred. This may be due to the acid chloride coordinating/chelating to the magnesium centre. How this then decomposes is not known.

The use of the methyl ester 3.12 (at room temperature) or ketone 3.10 (at $0{ }^{\circ} \mathrm{C}$ ) as electrophiles gave essentially no reaction. In each case, only trace amounts of $\mathbf{3 . 1 0}$ or triarylmethanol $\mathbf{1 . 6 5}$ respectively were observed. When DMPU was used as cosolvent with the ketone as electrophile, again, no reaction occurred. Transmetalating to cerium from the Grignard reagent was equally unsuccessful, returning the ketone and pyrimidine byproducts. In this case, a series of small peaks in the ${ }^{1} \mathrm{H}$ NMR spectrum between $\delta 5.00$ and 7.20 may indicate a small amount of addition to the pyridine or pyrimidine rings(s). However, no addition to the carbonyl group took place, as 1.65 was not present in the crude mixture.

Figure 3.4 summarises the attempted variations using the Grignard reagent 3.13. The failure to produce any of the desired triarylmethanol 1.65 meant that a new strategy for its synthesis would have to be developed. 
<smiles>COc1ccnc(Cl)c1C(=O)c1ccnc(SCC(=O)O)n1</smiles><smiles>COc1ccnc(Cl)c1C(=O)OCc1ccccc1</smiles><smiles>CSc1nccc(N(C)Cl)n1</smiles>

1. $\mathrm{CeCl}_{3}, \mathrm{THF}$,

3.13

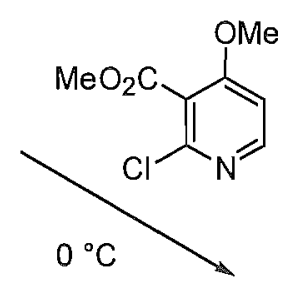

2.<smiles>COc1ccnc(Cl)c1C(=O)c1ccnc(SC)n1</smiles>

Figure 3.4 Attempted reactions with Grignard reagent 3.13. 


\subsection{An Altermative Synthesis of Triarylmethamol 1.65}

\subsubsection{Strategy}

Having explored a variety of options for the metalation of iodopyrimidine $\mathbf{2 . 1 2}$, most of which had met with resounding failure, a new strategy was developed for the synthesis of 1.65 (Figure 3.5b). The new approach employs an alternative disconnection to that shown in Figure 3.5a. These two approaches can be compared to the general approaches to triarylmethanols depicted in Figure 2.2b and $\mathbf{c}, \mathrm{p} 32$. The disconnection shown in Figure $\mathbf{3 . 5 b}$ would generate the symmetrical biaryl ketone $\mathbf{3 . 1 5}$ and a lithiated pyridine 3.16. It was hoped that the use of the alternative aryllithium species would allow higher temperatures to be used than was possible for the unstable pyrimidinyllithium species 1.66. The latter would still be required, however, for generation of the symmetrical ketone by reaction with a suitably reactive electrophile (Figure $3.5 b)$.

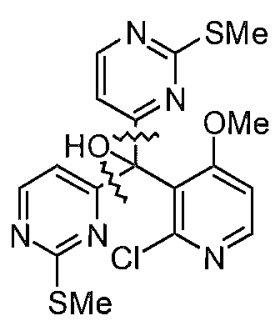

a<smiles>COc1ccnc(Cl)c1C(O)(c1ccnc(SC)n1)c1ccnc(SC)n1</smiles>

$\mathbf{b}$<smiles>CSc1nccc(C(=O)c2ccnc(SC)n2)n1</smiles>

3.15<smiles>COc1ccnc(Cl)c1Cl</smiles>

3.16

$$
\downarrow
$$

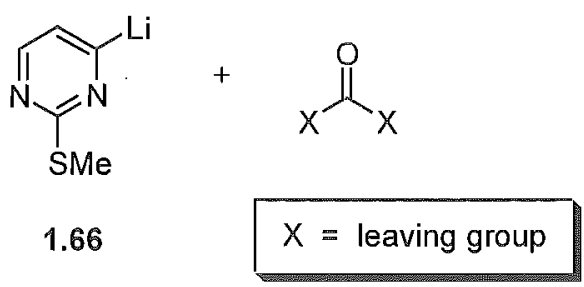

Figure 3.5 (a) Previous disconnection of 1.65; (b) New approach to triarylmethanol 1.65. 


\subsubsection{Synthesis of the Pyridine}

A literature search of 2-chloro-4-methoxypyridine (3.8), the precursor required for the generation of lithiated pyridine 3.16, revealed only a few reports of its synthesis and use. ${ }^{103,104,105}$ Encouragingly, these reports detailed the lithiation of 3.8 at $\mathrm{C} 3$ using either LTMP ${ }^{104}$ or LDA ${ }^{105}$ at $-78^{\circ} \mathrm{C}$, to form the required lithio-species 3.16. However, it was stated that attempted lithiation of 3.8 using alkyllithium reagents $(n-, s-$ or $t$-BuLi) failed due to competing substitution or halogen-metal exchange. ${ }^{105}$

While 3.8 was observed as a minor side-product in the formation of the pyridine carboxylic ester 3.7 (Scheme 3.2), a more practical route was required for the new approach to triarylmethanol 1.65. Finger and Starr have produced 2-chloro-4-nitropyridine (3.17) in three steps from 2chloropyridine $^{106}$ (Scheme 3.5). The nitro group of $\mathbf{3 . 1 7}$ has been substituted with sodium methoxide in refluxing methano $1^{103}$ or dioxane ${ }^{105}$ to produce 3.8 in $77 \%$ and $55 \%$ yields respectively.

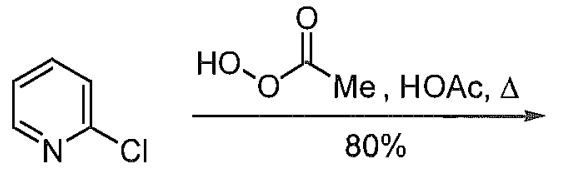<smiles>CO[n+]1ccccc1Cl</smiles>

$$
\underset{69 \%}{\stackrel{\mathrm{H}_{2} \mathrm{SO}_{4}, \mathrm{HNO}_{3}, \Delta}{\longrightarrow}}
$$<smiles>COc1ccnc(Cl)c1</smiles>

3.8<smiles>CO[n+]1ccc([N+](=O)[O-])cc1Cl</smiles>

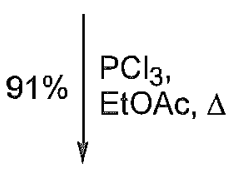

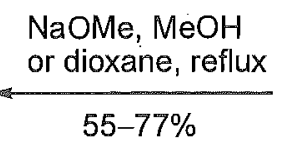

$55-77 \%$<smiles>O=[N+]([O-])c1ccnc(Cl)c1</smiles>

3.17 
Walters and Shay have reported that 3.17 could be produced in one step by treating 4 nitropyridine- $N$-oxide with a mixture of $\mathrm{POCl}_{3}$ and $\mathrm{PCl}_{5}{ }^{104}$ However, no experimental details were given and attempts to repeat the reaction in this Department failed to produce any of the chloropyridine $3.8 .^{107}$

In the end, 4-nitropyridine- $N$-oxide was converted to the desired 2-chloro-4-methoxypyridine by a three-step procedure, which is outlined in Scheme 3.6. The nitro group was displaced by sodium methoxide, following Ochiai's general procedure, ${ }^{108}$ to give 4-methoxypyridine- $N$-oxide (3.18) in 75\% yield." Attempts to directly convert the $N$-oxide 3.18 to 2 -chloro-4methoxypyridine (3.8) using $\mathrm{POCl}_{3}{ }^{109}$ or mixtures of $\mathrm{POCl}_{3}$ and $\mathrm{PCl}_{5}$ failed. Oxalyl chloride with catalytic or stoichiometric DMF was also ineffective, while refluxing in thionyl chloride resulted in deoxygenation to give 4-methoxypyridine in $82 \%$ yield. However, the $N$-oxide 3.18 could be isomerised to 4-methoxy-2-pyridone (3.19) in 61\% yield, following a literature procedure. $^{110}$ This involved heating 3.18 in refluxing acetic anhydride and subsequent hydrolysis of the acetoxy intermediate. The pyridone was converted to 3.8 by heating in $\mathrm{POCl}_{3}{ }^{111}$ Inconsistent results were obtained when this reaction was performed at reflux. Inattention to the temperature of the oil bath was the cause. It is thought that as the reaction progresses, phosphoric acid (or a related phosphorus-product) is produced which raises the reflux temperature of the mixture. If a particularly hot oil bath is used, the internal temperature rises significantly, resulting in lower yields of product. When the oil bath temperature was maintained at $95^{\circ} \mathrm{C}$, consistent results were obtained and 3.8 was isolated in $79 \%$ yield.

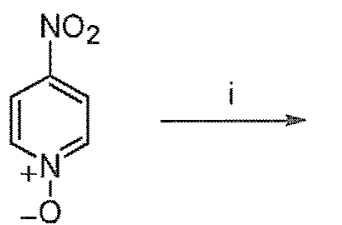

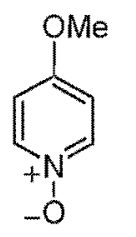

3.18

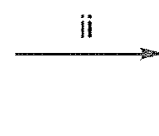

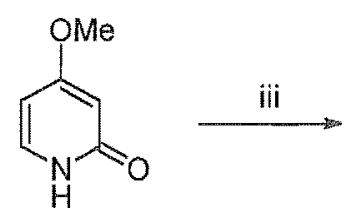

3.19<smiles>COc1ccnc(Cl)c1</smiles>

3.8

Scheme 3.6 Reagents and yields: (i) $\mathrm{NaOMe}$ (1 equiv)/MeOH/rt (75\%); (ii) $\mathrm{Ac}_{2} \mathrm{O} / \mathrm{reflux}$ then aq $\mathrm{MeOH} / \mathrm{rt} \mathrm{(61 \% );} \mathrm{(iii)} \mathrm{POCl}_{3} / 95^{\circ} \mathrm{C}(79 \%)$.

\footnotetext{
"While 3.18 is commercially available from Aldrich Chemical Company, it was convenient to prepare it from 4nitropyridine- $N$-oxide, which is also available from these suppliers at a substantially lower cost.
} 


\subsubsection{Synthesis of the Symmetrical Ketone}

The other component required for the synthesis of triarylmethano1 1.65 is the symmetrical ketone 3.15. Symmetrical biaryl ketones have been formed by metal carbonyl-catalysed carbonylations of aryl halides ${ }^{112}$ or aryl mercuric species, ${ }^{113}$ or by reaction of aryllithium reagents with an appropriate carbonyl compound (e.g. diethyl carbonate). ${ }^{114}$ Due to our familiarity with the pyrimidinyl lithio-species $\mathbf{1 . 6 6}$, the latter option was adopted.

Lithiation of $\mathbf{2 . 1 2}$ in the usual manner $\left(n-\mathrm{BuLi}, \mathrm{THF},-95^{\circ} \mathrm{C}\right.$ in the reactor described in Figure 2.8) produced the lithiated pyrimidine 1.66 (Scheme 3.7). A solution of diethyl carbonate (0.5 equivalents) in THF was added slowly and the reaction was allowed to warm slowly to room temperature. Between -75 and $-70{ }^{\circ} \mathrm{C}$ the dark brown solution took on a cherry-red colour, which intensified to deep violet on further warming. The major component of the crude mixture did indeed appear to be the desired ketone 3.15, judging by ${ }^{1} \mathrm{H}$ NMR spectroscopy. The product gave only two aromatic signals - a pair of doublets at $\delta 7.54$ and 8.79. These values compare well to the pyrimidine signals of the non-symmetrical ketones 2.11 and 3.10. A singlet was also present at $\delta 2.51$, representing the thiomethyl protons. Pleasingly, only very small amounts of the lithiation byproducts $\mathbf{2 . 2 0}$ and 2.21 were present and 2.22 was not detected in the mixture at all, in contrast to the use of $\mathbf{2 . 1 2}$ in the syntheses of triarylmethanols 2.3 and $\mathbf{1 . 6 5}$.

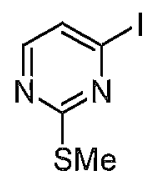

2.12
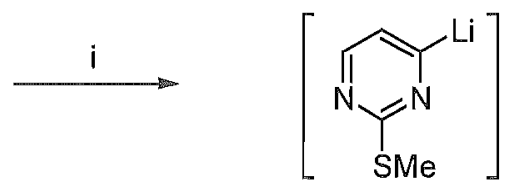

1.66
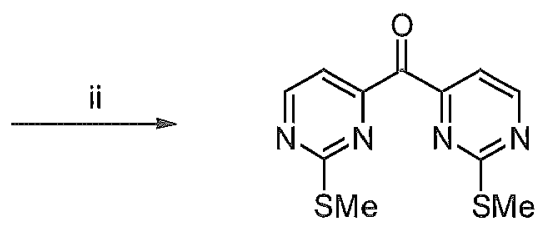

3.15

Scheme 3.7 Reagents and yields: (i) $n$-BuLi (1 equiv)/THF/-95 ${ }^{\circ} \mathrm{C}, 30 \mathrm{~min}$; (ii) diethyl carbonate $(0.5$ equiv)/THF/-95 ${ }^{\circ} \mathrm{C}$ to $\mathrm{rt}(53 \%)$.

When subjected to flash chromatography, the product exhibited unusual behaviour, smearing badly on silica (although it did elute, given enough solvent) and sticking to alumina (only eluting 
with methanol/ethyl acetate mixtures). After some experimentation, it was found that it was most convenient to distil the crude product under high-vacuum in a Kugelrohr apparatus (3.15 melts at $106^{\circ} \mathrm{C}$ and distils at $160^{\circ} \mathrm{C}, 0.03 \mathrm{~mm} \mathrm{Hg}$ ), and then to remove the remaining impurities by flash chromatography on silica. In this manner, the pure ketone could be isolated in $53 \%$ yield.

When ethyl chloroformate was used as the electrophile in place of diethyl carbonate, the ketone was not produced as cleanly. Pyrimidine byproducts 2.20 and 2.21 were both present in greater quantities than when diethyl carbonate was used, and a new product was observed. Although this product was not isolated, it is most likely the symmetrical triarylmethanol $\mathbf{3 . 2 0}$, produced from the addition of three equivalents of the lithio-species $\mathbf{1 . 6 6}$ to ethyl chloroformate. It displays a pair of doublets in the ${ }^{1} \mathrm{H}$ NMR spectrum at $\delta 7.49$ and 8.48 and a broad peak at $\delta$ 6.15. These values compare fairly well to the pyrimidine and $\mathrm{OH}$ signals of triarylmethanols $\mathbf{2 . 3}$ and 1.65 .

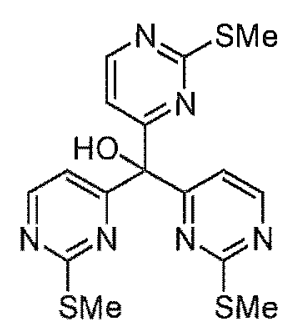

3.20

When the Grignard reagent 3.13 was used in place of $\mathbf{1 . 6 6}$, the reaction with diethyl carbonate gave only small amounts of the ketone, with deiodinated pyrimidine 2.20 and bipyrimidine 2.21 being the major products. If ethyl chloroformate was the electrophile, no ketone could be detected. Thus, despite the greater stability of the magnesium species, the lithiated pyrimidine $\mathbf{1 . 6 6}$ has proven to be the superior reagent in the author's experience.

\subsubsection{Formation of the Triarylmethanol 1.65}

As has been mentioned, 2-chloro-4-methoxypyridine (3.8) has been lithiated between the chlorine and the methoxy group using either $\mathrm{LTMP}^{104}$ or $\operatorname{LDA}^{105}$ at $-78^{\circ} \mathrm{C}$, to give the lithiated pyridine 3.16 (Scheme 3.8). In both of these reports, the lithio-species thus generated was used 
as a precursor to 4-methoxy-2,3-pyridyne (3.21) -warming the reaction to room temperature results in elimination of LiCl. Evidence of pyridyne formation is seen by in situ trapping with furan to give the Diels-Alder adduct $\mathbf{3 . 2 2}$.

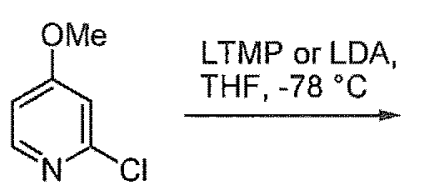

3.8<smiles>COc1ccnc(Cl)c1Cl</smiles>

3.16

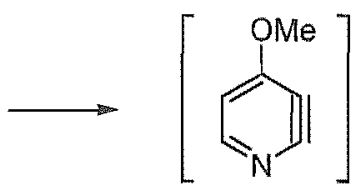

3.21

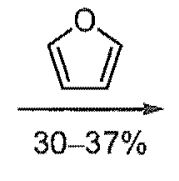<smiles>COc1ccnc2c1C1C=CC2O1</smiles>

3.22

Scheme 3.8

As it was the lithio-species $\mathbf{3 . 1 6}$ that we required, the formation of the pyridyne was the cause of some concern - it was not known how readily elimination of $\mathrm{LiCl}$ from 3.16 would occur. It was hoped that the lithio-species would be stable at $-78^{\circ} \mathrm{C}$ and reactive enough to add to the symmetrical ketone $\mathbf{3 . 1 5}$.

It should be noted that with either LTMP or LDA, starting material was recovered from the reaction ( $37 \%$ in the case of LDA). Comins and LaMunyon have found that when LDA is used to deprotonate ortho to the methoxy group of 2-,3- and 4-methoxypyridines, only low amounts of deuterium incorporation are obtained when the reaction is quenched with $\mathrm{D}_{2} \mathrm{O}{ }^{41}$ This was ascribed to the existence of an equilibrium between LDA and the metalated pyridine, in which the position of equilibrium lies in favour of LDA and the pyridine (Figure 3.6). Clearly, LDA was too weak a base in this situation.
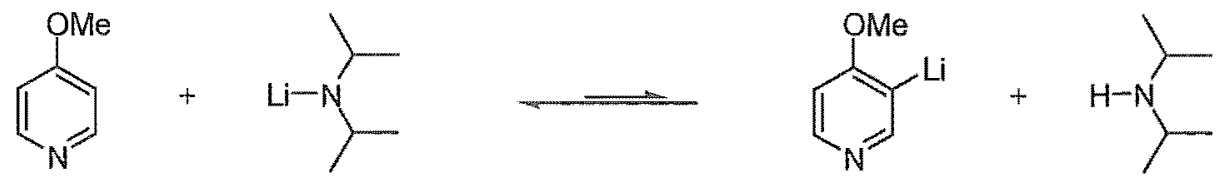

Figure 3.6 Equilibrium between 4-methoxypyridine/LDA and the metalated pyridine/ diisopropylamine. 
In the case of 2-chloro-4-methoxypyridine (3.8), the inductive effect of the chlorine would increase the acidity of $\mathrm{H} 3$ relative to $\mathrm{H} 3$ of 4-methoxypyridine. However, the fact that starting material was isolated when either LTMP or LDA were used suggests that these bases do not effect complete deprotonation of 3.8. For this reason, it was decided to examine the metalation of 2-chloro-4-methoxypyridine (3.8) with a variety of alkyllithium bases, even though Connon and Hegarty have claimed that $n-, s$ - and $t$-BuLi could not be used to lithiate $3.8 .^{105}$

As mesityllithium has been successfully used by Comins to deprotonate 4-methoxypyridine, this base was applied to the present situation. Comins' optimum conditions for metalation involve stirring the reagents at $-23{ }^{\circ} \mathrm{C}$ for $3 \mathrm{~h}$, followed by introduction of the electrophile. However these conditions would not be suitable for 2-chloro-4-methoxypyridine (3.8) as pyridyneformation is likely to occur at this temperature. Therefore, 3.8 and mesityllithium (1.1 equivalents) were stirred at $-78^{\circ} \mathrm{C}$ for $1 \mathrm{~h}$ and quenched with $\mathrm{CH}_{3} \mathrm{OD}$. Under these conditions, only starting material was isolated-no deuterium was incorporated into the pyridine nucleus. Presumably, this very hindered base requires higher temperatures to deprotonate the pyridine and is therefore unsuitable in the present situation.

When $t$-BuLi was used at $-78^{\circ} \mathrm{C}$, the major component of the mixture appeared to be the result of nucleophilic addition ( $85 \%$ of the mixture, judging by ${ }^{1} \mathrm{H}$ NMR spectroscopy). The addition product was not isolated, but is presumed to be dihydropyridine $\mathbf{3 . 2 3}$, in which the $t$-butyl group has added to the unsubstituted azomethine bond. This is a surprising result, based on the observation of Connon and Hegarty that exclusive lithium-chlorine exchange occurred between $t$-BuLi and 2-chloro-4-phenoxypyridine. ${ }^{105}$ However, some metalation had occurred in the present case, with about $8 \%$ of the deuterated pyridine 3.24 being present in the mixture, in addition to a similar amount of starting material $\mathbf{3 . 8}$.

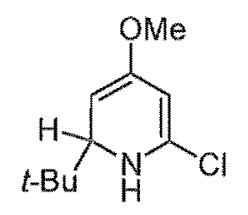

3.23<smiles>[2H]c1c(OC)ccnc1Cl</smiles>

3.24 
When $n$-BuLi was employed as metalating agent, the situation was markedly improved. After stirring the pyridine and $n$-BuLi (1.1 equiv) in THF at $-78^{\circ} \mathrm{C}$ for $1 \mathrm{~h}$ and quenching with $\mathrm{CH}_{3} \mathrm{OD}$, deuterated pyridine 3.24 was produced in $84 \%$ yield (judging by ${ }^{1} \mathrm{H}$ NMR spectroscopy). Some starting material $(\sim 7 \%)$ was still present and a small amount of addition product $(\sim 9 \%)$ was also observed. When the lithiation was repeated at lower temperature $(-95$ ${ }^{\circ} \mathrm{C}$ ), the addition product was virtually eliminated, although some starting material was still present $(\sim 13 \%)$. Pleasingly, no products indicative of pyridyne formation, or of lithiation at C5 were observed, either at -78 or $-95^{\circ} \mathrm{C}$. Over the course of the lithiation at $-78{ }^{\circ} \mathrm{C}$, an intense dark cherry colour developed, which may be due to trace amounts of pyridyne. However, at -95 ${ }^{\circ} \mathrm{C}$, the colour of the solution never progressed any further than orange.

These findings indicate that $n-\mathrm{BuLi}$ is an effective base for the deprotonation of $\mathbf{3 . 8}$, in contrast to claims otherwise. It remained to see if the lithiated pyridine $\mathbf{3 . 1 6}$ would react with the ketone 3.15 to provide the desired triarylmethanol. Pleasingly, when the two reagents were stirred at $78{ }^{\circ} \mathrm{C}$ for $10 \mathrm{~h}$, followed by quenching with methanol at this temperature, triarylmethanol $\mathbf{1 . 6 5}$ was isolated in $57 \%$ yield-a significant improvement on the previous method. It was subsequently found that $3.5 \mathrm{~h}$ at $-78^{\circ} \mathrm{C}$ was sufficient time for reaction to occur, as quenching with $\mathrm{CH}_{3} \mathrm{OD}$ gave no deuterated pyridine 3.24. However, it was observed that a significant amount of unreacted pyridine 3.8 was returned ( $\sim 35 \%$ by ${ }^{1} \mathrm{H}$ NMR analysis). When $\mathbf{3 . 8}$ was freshly distilled prior to use, however, the amount of pyridine remaining after quenching the reaction was reduced to $\sim 12 \%$. Despite the incomplete consumption of 3.8 , triarylmethanol 1.65 was isolated in a yield of 76\%, based on ketone 3.16 (Scheme 3.9). The presence of unreacted 3.8 may indicate that the metalation step required longer times or excess $n$-BuLi. However, neither of these (nor the combination of both) had any effect on the amount of 3.8 which remained. Therefore, it may be that 3.8 is produced on quenching of the lithiated pyridine 3.16 with an adventitious proton source. In any case, recovery and recycling of starting materials would add to the efficiency of the process. 


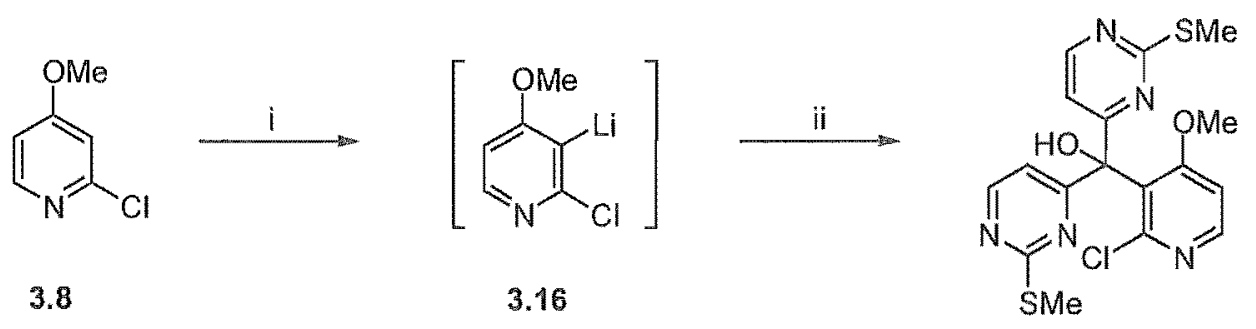

Scheme 3.9 Reagents and yields: (i) $n$-BuLi (1 equiv) $/ \mathrm{THF} /-95$ to $-78{ }^{\circ} \mathrm{C}, 1 \mathrm{~h}$; (ii) $3.15(0.93$ equiv)/THF $/-95$ to $-78^{\circ} \mathrm{C}, 3.5 \mathrm{~h}(76 \%)$.

\subsubsection{Summary}

Using the lithiation methodology established for the synthesis of triarylmethanol 2.3 (Chapter Two), it was difficult to obtain consistent results and the desired triarylmethanol 1.65 was produced in a maximum of $15 \%$ yield. No improvement on the yield was seen when different metal-species (magnesium and cerium) were employed and consequently, the approach was abandoned.

The second approach involved reacting a lithiated pyridine $\mathbf{3 . 1 6}$ with a symmetrical bipyrimidinyl ketone 3.15 . The pyridine precursor 3.8 was generated in three steps from readily available 4-nitropyridine- $N$-oxide, while the ketone was formed by reaction of the lithiated pyrimidine $\mathbf{1 . 6 6}$ with diethyl carbonate. Conditions were found for the successful lithiation of 3.8 , and the resulting lithio-species 3.16 could be reacted with 3.15 to give the desired triarylmethanol in $76 \%$ yield. While an extra step is required in this synthesis of 1.65 compared to the former approach, the overall yield is much improved ( $40 \%$ from iodide 2.12 , compared to $<15 \%$ ). Importantly, this synthesis of $\mathbf{1 . 6 5}$ is a very reproducible process which gives consistently high yields, in contrast to the difficulties encountered in the former approach. 


\subsection{Formation of the Variolin Core 1.63}

Now that a reliable synthesis of the triarylmethanol 1.65 had been developed, its cyclisation to the variolin core could be investigated. Based on the successful use of the TFA/TES hydrogenolysis pair on the deoxy-system, this methodology formed the basis of our approach to the methoxy-containing variolin core $\mathbf{1 . 6 3}$.

\subsubsection{TFA/TES "Ionic Hydrogenolysis" of Triarylmethanol 1.65}

\subsubsection{Preliminary Results}

The methoxy-containing triarylmethanol $\mathbf{1 . 6 5}$ was subjected to the same conditions (4 equivalents TFA, 8 equivalents TES, $70^{\circ} \mathrm{C}$ ) that had been optimal for the deoxy-system. One major component was observed in the ${ }^{1} \mathrm{H}$ NMR spectrum of the crude mixture, which, by all appearances was a rearrangement product, 3.25. This is analogous to compound 2.39, which was isolated as a side-product in the formation of the deoxy-variolin core 2.1 (Chapter Two). A minor product appeared to be the desired core structure 1.63. Flash chromatography allowed the isolation of the two compounds in $54 \%$ and $9 \%$ yields respectively.

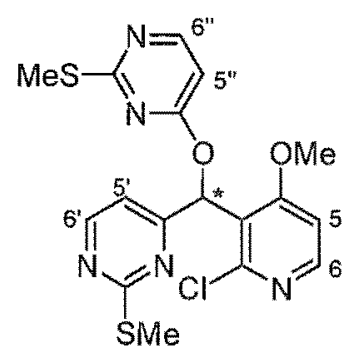

3.25

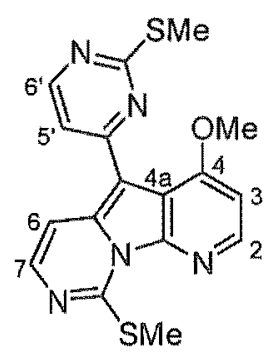

1.63

Spectroscopic analysis of the purified products confirmed their structures. The uncyclised ether 3.25 displays two separate thiomethyl signals in its ${ }^{1} \mathrm{H}$ NMR spectrum at $\delta 2.36$ and 2.44 , and a methoxy signal at $\delta 3.84$. Each of these peaks integrate for three protons. In the aromatic region, seven well-separated signals were observed, each integrating for one proton (Figure 3.7). Most prominent was the singlet corresponding to the central methine proton, appearing at $\delta 7.79$. 
The remaining signals (three pairs of doublets) correspond to the protons on the three, nonequivalent aromatic rings.

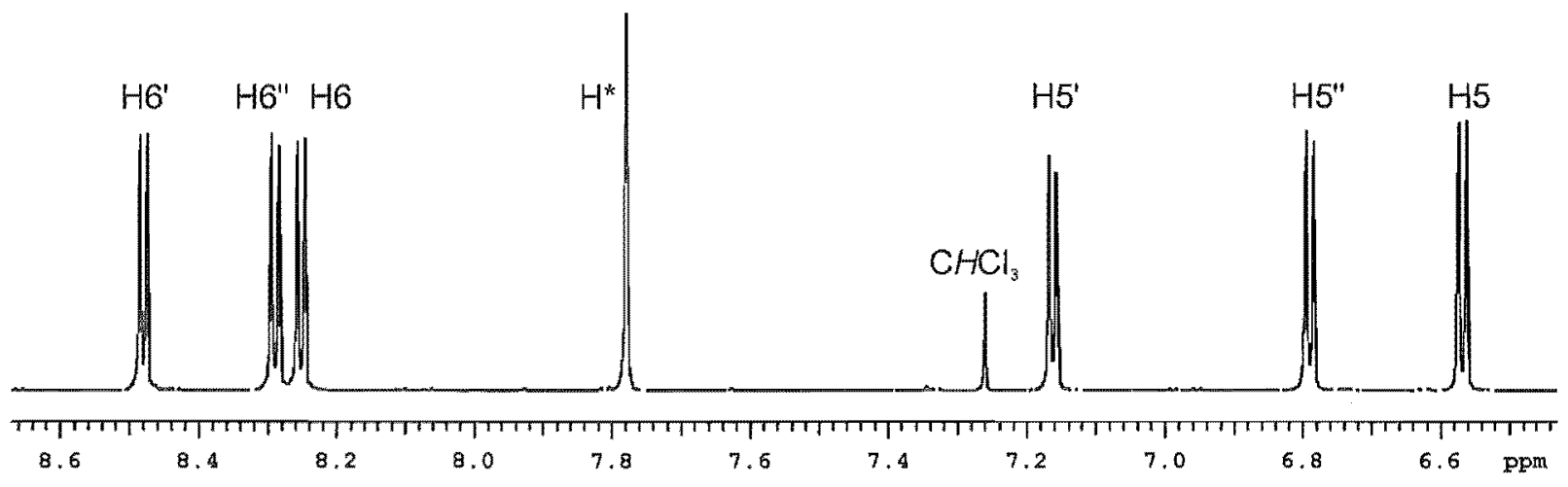

Figure 3.7 Aromatic region of the $500 \mathrm{MHz}{ }^{1} \mathrm{H}$ NMR spectrum of $\mathbf{3 . 2 5}$.

The ${ }^{1} \mathrm{H}$ NMR signals of the variolin core 1.63 match very well to those of the deoxymequivalent 2.1 (see Figure 3.8). The main differences lie in the pyridine ring, due to the methoxy group at C4. As there are only two protons on this ring, rather than three in 2.1 , the signals appear as doublets-not doublets-of-doublets as seen in 2.1. The $+\mathrm{R}$ effect of the methoxy group shifts the signal of $\mathrm{H} 30.6 \mathrm{ppm}$ further upfield than is seen in the deoxy-core 2.1. The signals for $\mathrm{H} 2, \mathrm{H} 6$ and $H 7$ of 1.63 all appear $\sim 0.1$ ppm upfield of the same signals of 2.1 , while the signals for the pendant pyrimidine ring ( $\mathrm{H}^{\prime}$ and $\mathrm{H}^{\prime}$ ) are relatively unchanged. As with 2.1, $\mathrm{H} 6$ appears more downfield than $\mathrm{H} 7$, presumably for the same reasons-a combination of the electronic nature of the fused pseudo-pyrimidine ring and the anisotropic deshielding effect associated with the aromatic ring current of the pendant pyrimidine ring. 


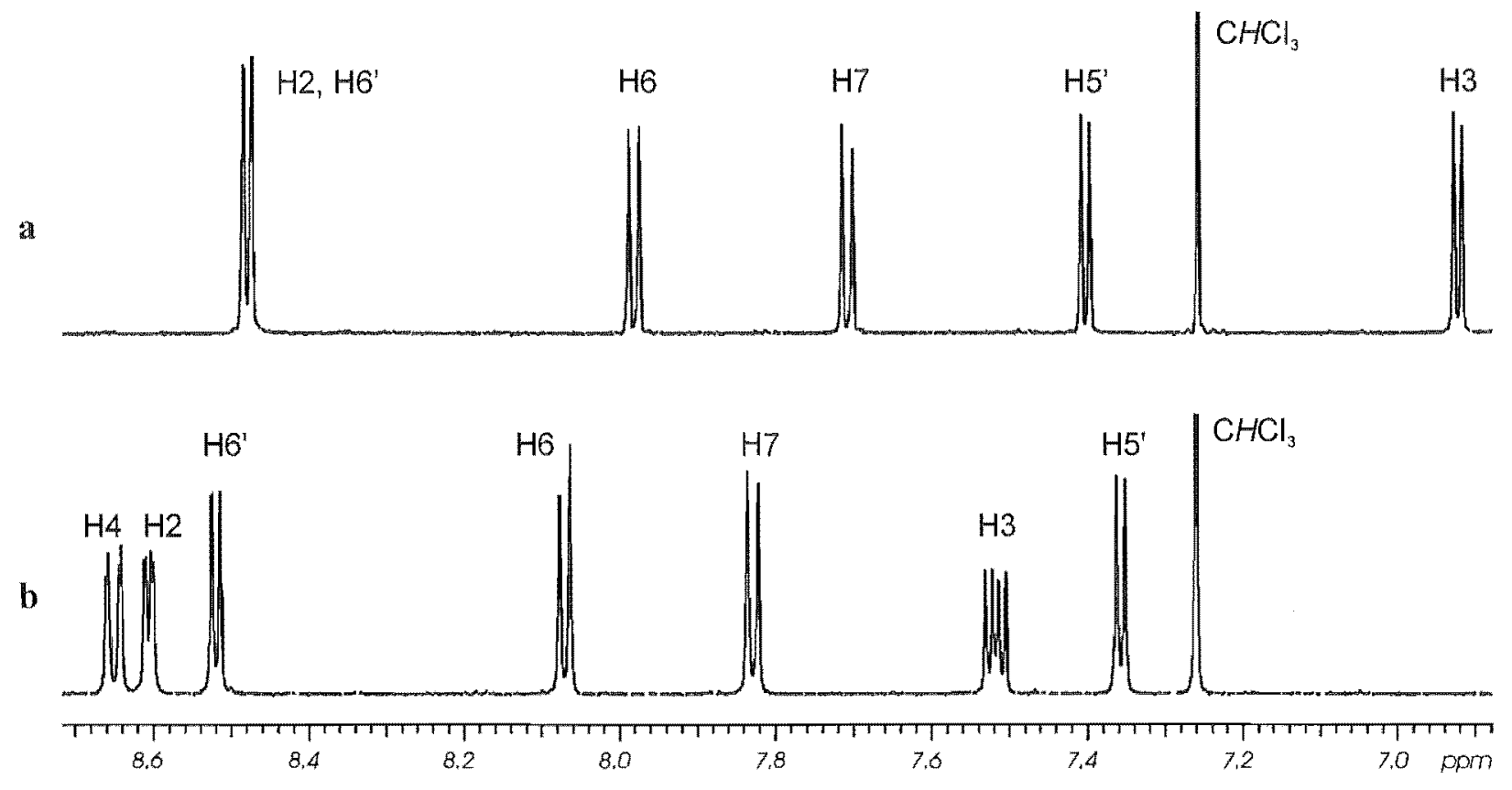

Figure 3.8 Aromatic regions of the $500 \mathrm{MHz}{ }^{1} \mathrm{H}$ NMR spectra of (a) the variolin core 1.63, and (b) the deoxy-equivalent 2.1 .

In the ${ }^{13} \mathrm{C}$ NMR spectrum, no major differences are seen between the two compounds except for positions 3, 4 and 4a. Specifically, C3 and C4a shift upfield by 19 and 10 ppm respectively upon introduction of the electron-donating methoxy group, while $\mathrm{C} 4$ moves downfield by $32 \mathrm{ppm}$ due to the -I effect of the oxygen.

It should also be pointed out that MS analysis confirmed the molecular formula of 1.63. While comparison of the NMR chemical shifts of 1.63 and the deoxy-equivalent 2.1 provided good evidence for the structure of 1.63 , MS analysis was necessary to prove the absence of oxygen in the formula. Thus, the product must be the desired variolin core 1.63 , and not a structure analogous to the oxygen-containing compounds 2.42 and 2.44 discussed in Chapter Two.

The low yield of 1.63 was rather disappointing. However, it must be noted that the reaction proceeded much more cleanly than in the deoxy-system. Even with the best conditions for formation of the deoxy-variolin core 2.1, several other side-products (both identified and unidentified) were present in significant amounts. In the present system, however, any other 
side-products comprised only a very minor part of the mixture. It is interesting that no products analogous to 2.42 and 2.44 were detected in the reaction mixture.

\subsubsection{Optimisation of the Yield of the Variolir Core 1.63}

A comprehensive series of reactions were carried out to determine the best conditions for formation of the variolin core 1.63. The use of a solvent, less TFA and longer reaction times all had beneficial effects on the yield of 1.63 . However, the greatest improvement was observed when the reaction was conducted at higher temperatures in a sealed tube. The optimum conditions found consisted of heating 1.65 (100 mg) with TFA (2 equivalents), TES (8 equivalents) and 1,2-dichloroethane (DCE) $(0.5 \mathrm{~mL})$ in a sealed tube at $100{ }^{\circ} \mathrm{C}$ for $43 \mathrm{~h}$. In this manner, the variolin core was isolated as a yellow solid in $47 \%$ yield (Scheme 3.10). The uncyclised ether $\mathbf{3 . 2 5}$ was the only other significant component of the reaction, being isolated in $28 \%$ yield.<smiles>COc1ccnc(Cl)c1C(O)(c1ccnc(SC)n1)c1ccnc(SC)n1</smiles>

1.65

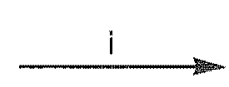<smiles>COc1ccnc(Cl)c1C(Oc1ccnc(SC)n1)c1ccnc(SC)n1</smiles>

3.25

Scheme 3.10 Reagents and yields: (i) TFA ( 2 equiv)/TES (8 equiv)/DCE/100 ${ }^{\circ} \mathrm{C}(1.6347 \%, 3.25$, $28 \%$ ).

As explained for the deoxy-system in Chapter Two, it is assumed that 3.25 arises from intramolecular attack of the hydroxyl group at $\mathrm{C} 4$ of a pyrimidine ring (Figure 3.9). Presumably, protonation of the pyrimidine ring aids this process. Two strategies were devised to minimise/block this pathway. It was thought that using a weaker acid would favour protonation of the more basic pyridine ring over the pyrimidine ring. This should favour formation of the 
desired variolin core 1.63 over the uncyclised product 3.25 . However, conducting the reaction in acetic acid (without DCE as solvent) gave only starting material.<smiles>CCC(O)(c1ccnc(SC)n1)c1c(OC)ccnc1Cl</smiles>

1.65<smiles>COc1ccnc(Cl)c1C1(c2ccnc(SC)n2)OC12C=CN=C(SC)N2</smiles>

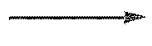<smiles>COc1ccnc(Cl)c1C(Oc1ccnc(S(C)(=O)=O)n1)c1ccnc(SC)n1</smiles>

3.25

Figure 3.9 Proposed mechanism of formation of 3.25 .

\subsubsection{An Alternative Substrate for the TFA/TES Reaction}

\subsubsection{Improved Synthesis of the Variolin Core 1.63}

The alternate strategy was to block the hydroxyl group's ability to act as a nucleophile by protecting it in some form. As discussed in Chapter Two, functionalisation of triarylmethanol 2.3 as either the xanthate or methyloxalyl ester was unreliable, at best. The present system poses a more difficult situation, being more hindered around the hydroxyl group. However, the alcohol may be able to be protected with a simpler, more stable group such as an acetyl group. If hydroxyl protection could be achieved, the pathway shown in Figure 3.9 would be effectively blocked. Cyclisation to the variolin core, however, should not be adversely affected.

Acetylation of triarylmethanol $\mathbf{1 . 6 5}$ was effected with acetic anhydride, triethylamine and catalytic 4-(dimethylamino)pyridine (DMAP) in $\mathrm{CH}_{2} \mathrm{Cl}_{2}$. Acetylation of alcohols under these conditions usually proceeds with ease at room temperature. In this case, however, the conversion proved to be a very slow process, even at reflux. Several further additions of reagents were required throughout the course of the reaction. After eight days, the conversion 
was about $97 \%$ complete, judging by ${ }^{1} \mathrm{H}$ NMR spectroscopy. The sluggishness of this reaction is testimony to the extremely hindered nature of the central hydroxyl group of this compound.

The nucleophilicity of the triarylmethanol $\mathbf{1 . 6 5}$ should be increased if the alcohol was deprotonated to the alkoxide. As the required alkoxide is generated in the synthesis of the triarylmethanol, quenching this reaction at $-78^{\circ} \mathrm{C}$ with acetyl chloride may provide a method of producing the acetate without the need for an extra step. Pleasingly, using the same conditions for formation of triarylmethanol 1.65 , except that the alkoxide was quenched at $-78^{\circ} \mathrm{C}$ with acetyl chloride, triarylmethylacetate $\mathbf{3 . 2 6}$ was readily produced, with the alcohol not being detected (Scheme 3.11). After chromatography, acetate $\mathbf{3 . 2 6}$ was isolated in $68 \%$ yield.<smiles>COc1ccnc(Cl)c1</smiles>

3.8<smiles>COc1ccnc(Cl)c1C(O)(c1ccnc(SC)n1)c1ccnc(SC)n1</smiles>

1.65

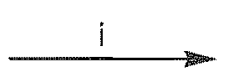<smiles>COc1ccnc(Cl)c1C(O)(c1ccnc(SC)n1)c1ccnc(SC)n1</smiles>

3.26

ii

Scheme 3.11 Reagents and yields: (i) $\mathrm{Ac}_{2} \mathrm{O}$ (3 equiv) $/ \mathrm{Et}_{3} \mathrm{~N}$ (2 equiv)/cat. DMAP/CH $\mathrm{Cl}_{2} / \mathrm{reflux}$ ( $97 \%$ conversion after 8 days); (ii) $n$-BuLi ( 1 equiv) $/ \mathrm{THF} /-90^{\circ} \mathrm{C}$ to $-78^{\circ} \mathrm{C}, 1$ h then 3.15 (1 equiv) $/ \mathrm{THF} /-90^{\circ} \mathrm{C}$ to $-78^{\circ} \mathrm{C}, 3 \mathrm{~h}$ then $\mathrm{AcCl}\left(4\right.$ equiv) $/-78{ }^{\circ} \mathrm{C}$ to rt $(68 \%)$.

The ${ }^{1} \mathrm{H}$ NMR spectrum of 3.26 is similar to that of the alcohol 1.65. A new peak at $\delta 2.30$ was observed for the acetyl protons, while the $\mathrm{OH}$ peak of 1.65 had disappeared. Small shifts (up to $0.2 \mathrm{ppm}$ ) were seen for the other signals. Two extra peaks were noted in the ${ }^{13} \mathrm{C}$ NMR spectrum for the carbonyl and methyl carbons, while the IR spectrum confirmed the presence of the ester group with a strong peak at $1755 \mathrm{~cm}^{-1}$. In the EIMS spectrum, the molecular ion $(\mathrm{m} / \mathrm{z} 463)$ was barely detectable, with strong peaks at $m / z 428(-\mathrm{Cl})$ and $421(-\mathrm{Ac},+\mathrm{H})$. The base peak, however, was at $m / z 369$, which corresponds to the loss of chlorine and the acetoxy group. It is tempting to speculate that this ion possesses the structure of the variolin core 1.63 , and it was 
hoped that this facile fragmentation in the mass spectrometer would be reproduced in the forthcoming reaction,

To this end, the acetate 3.26 was treated to the same conditions that were used for reaction of the triarylmethanol 1.65 ( 2 equivalents TFA, 8 equivalents TES, DCE, sealed tube). However, before the oven had warmed to $100^{\circ} \mathrm{C}$, it was noted that the visible appearance of the mixture was quite different to what had previously been observed. The mixture had developed a dark yellow-brown colour which had come to be associated with formation of the variolin core. In addition, an orange-brown solid had precipitated out of solution - also indicative of the variolin core. What was most surprising was that these observations were noted after just $0.5 \mathrm{~h}$ at $80^{\circ} \mathrm{C}$ or less! The reaction was left at $80^{\circ} \mathrm{C}$ for a total of $26 \mathrm{~h}$ and, after cooling to room temperature, was worked up as usual. Other than triethylsilyl byproducts, the crude material consisted of essentially pure variolin core structure $\mathbf{1 . 6 3}$ ! No trace of the uncyclised product $\mathbf{3 . 2 5}$ could be detected. The product was obtained in a pure state in $75 \%$ yield, after flash chromatography on silica (Scheme 3.12).<smiles>COc1ccnc(Cl)c1C(O)(c1ccnc(SC)n1)c1ccnc(SC)n1</smiles>

3.26

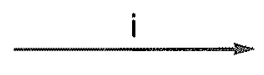

1.63

Scheme 3.12 Reagents and yields: (i) TFA (2 equiv)/TES ( 8 equiv)/DCE/80 ${ }^{\circ} \mathrm{C}$, sealed tube $(75 \%)$.

This gratifying result demonstrates the value of positively identifying unwanted side-products of a reaction, in order that steps may be taken to minimise/eliminate their formation. However, it was apparent that acetylation of the alcohol did not just block nucleophilic attack of oxygen at the pyrimidine ring. It also greatly increased the rate at which 1.63 formed.

\footnotetext{
"It has subsequently been shown by Jonathan Hill (of this Department) that this methodology is also applicable to the deoxy-system, allowing the core structure 2.1 to be isolated in $70 \%$ yield.
} 


\subsubsection{An Intermediate in the Formation of 1.63 ?}

Some insights into the mechanism of cyclisation were gained when acetic acid was used in place of TFA. When acetic acid was used, the reaction was visibly slower than with TFA, however, the variolin core $1.63 \mathrm{did}$ form, in $67 \%$ yield after chromatography. It should be reiterated that no reaction occurred when acetic acid was used with the triarylmethanol 1.65. A minor product of this reaction displayed quite unusual signals in its ${ }^{1} \mathrm{H}$ NMR spectrum. A combination of reversed phase and normal silica flash chromatography allowed isolation of the product " $\mathbf{Y}$ " in sufficient purity for characterisation by ${ }^{1} \mathrm{H},{ }^{13} \mathrm{C}$ and $2 \mathrm{D}$ NMR spectroscopy.

From the ${ }^{1} \mathrm{H}$ NMR spectrum, "Y" possesses four methyl groups-two thiomethyl groups, one methoxy group and one acetate. Most interesting were the two signals at $\delta 3.66$ and 4.33 ("a" and "b" in Figure 3.10a). These doublets-of-doublets were coupled to each other $(J=17 \mathrm{~Hz})$ and also to "c" at $\delta 6.50(J=3.4$ and $1.7 \mathrm{~Hz}$ respectively). Two pairs of doublets were also seen in the aromatic region. These features suggest a cyclised product (i.e. containing the pyridopyrrolopyrimidine core) with seven protons (apart from the various methyl protons).
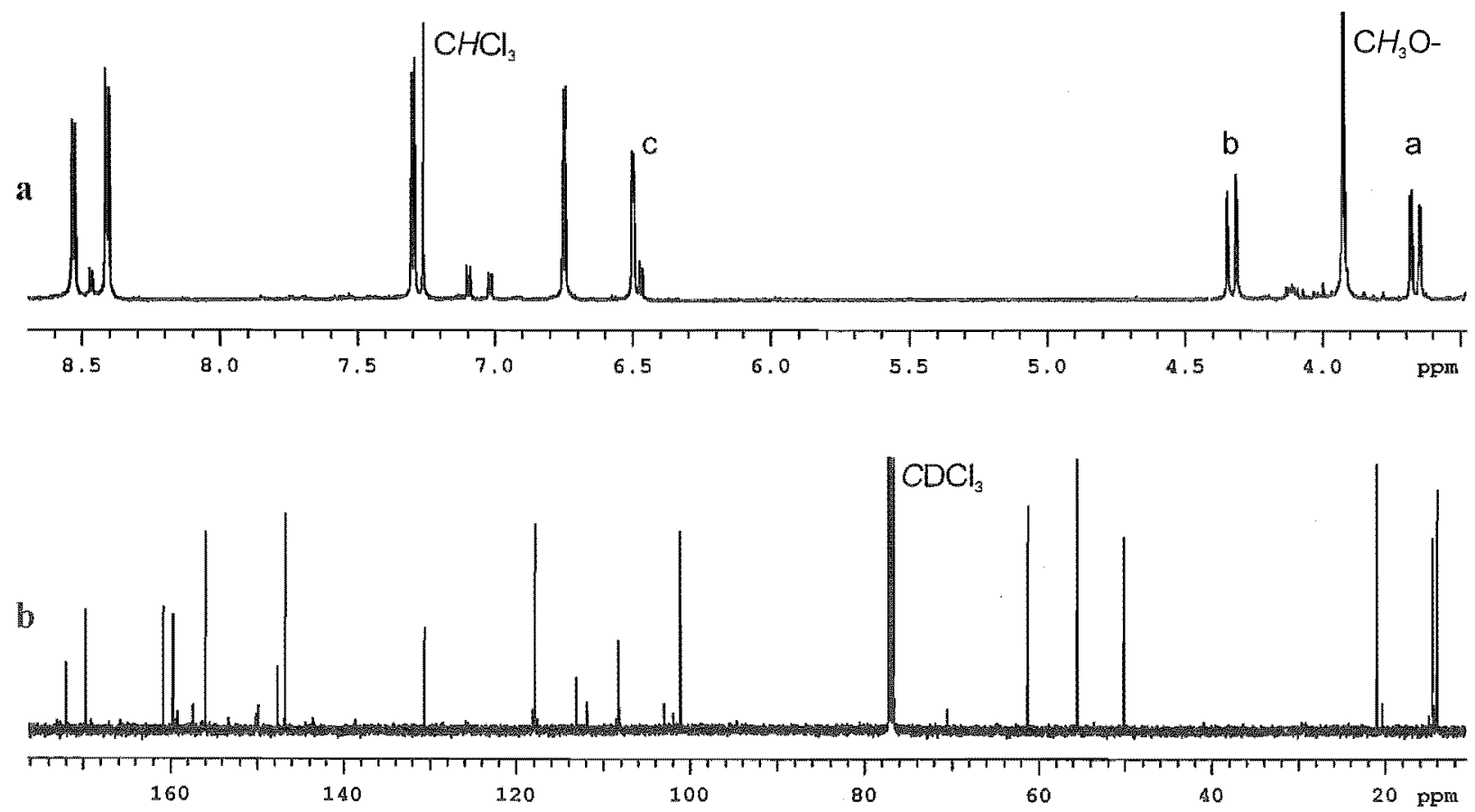

Figure 3.10 (a) A portion of the $500 \mathrm{MHz}^{1} \mathrm{H}$ NMR spectrum of $\mathbf{Y}$. Signals $\mathrm{a}, \mathrm{b}$ and $\mathrm{c}$ are all coupled to each other; (b) $126 \mathrm{MHz}{ }^{13} \mathrm{C}$ NMR spectrum of $\mathbf{Y}$. 
The large coupling constant of $17 \mathrm{~Hz}$ had never been observed in any other compounds associated with this work. This type of coupling is typical of geminal protons of a cyclic $-\mathrm{CH}_{2}-$ group. This was confirmed in the HSQC, which indicated that the two protons were attached to the same carbon at $\delta 50.2$. In the CIGAR experiment (the equivalent of a HMBC), these two protons showed correlations to a carbon at $\delta 150.1$, as did the protons of one of the thiomethyl groups (see Figure 3.11). This is convincing evidence that we are dealing with the fused pyrimidine ring of a pyridopyrrolopyrimidine system, in which two protons are attached to C7 and the carbon at $\delta 150.1$ would be C9. Invariably, the signal for C9 of these pyridopyrrolopyrimidine systems lies in the range $\delta 148-156$, while $\mathrm{C}^{\prime}$ (the equivalent carbon of the pendant pyrimidine ring) gives rise to a signal between $\delta 170$ and 173 (provided it is substituted with a thiomethyl group).

The 2D NMR data (see Figure $\mathbf{3 . 1 1}$ for some important correlations) did not locate the position of the acetate group. Initially, it was assumed that it is attached to $\mathrm{C} 5$ (which would require a double bond between $\mathrm{C} 5 \mathrm{a}$ and $\mathrm{C} 6$ ), as it is located at this position in the starting material. This would give $\mathbf{3 . 2 7}$ as the structure for $\mathbf{Y}$. However, the signal for C5 appears at $\delta 113.1$ (see Figure 3.10b), a value which surely indicates $\mathrm{sp}^{2}$ character. Additionally, the signal for C6 ( $\delta$ 61.3 ) is much too low for an alkene/aromatic carbon, but is reasonable for an oxygenated $\mathrm{sp}^{3}$ carbon centre. Structure $\mathbf{3 . 2 8}$ is, therefore, presented as an alternative structure for $\mathbf{Y}$, which fits the spectroscopic data more adequately.

Further evidence for this structure was obtained when the CIGAR experiment was re-run under different conditions. In HMBC and CIGAR experiments, the parameter ${ }^{\mathrm{n}} J_{\mathrm{CH}}$ represents the ${ }^{\mathrm{I}} \mathrm{H}$ ${ }^{13} \mathrm{C}$ coupling constant selected for in the experiment, where " $n$ " is the number of bonds separating the two nuclei. This parameter is typically set to $8.3 \mathrm{~Hz}$, which allows correlations to be observed for $\mathrm{n}=2$ or 3 bonds. However, some 2- or 3-bond correlations may not be observed with ${ }^{n} J_{\mathrm{CH}}=8.3$, so for more thorough investigations, an arrayed value of ${ }^{n} J_{\mathrm{CH}}=4$ and $9 \mathrm{~Hz}$ may be used. Correlations over 4 bonds are not usually seen, due to ${ }^{4} J_{\mathrm{CH}}$ coupling being very small. However, very small couplings can sometimes be observed if a lower value of ${ }^{n} J_{\mathrm{CH}}$ is used. When the experiment was repeated with ${ }^{\mathrm{n}} J_{\mathrm{CH}}=2 \mathrm{~Hz}$, some additional correlations became apparent. The most important of these was a weak, but real, correlation from the acetate protons 
to $\mathrm{C} 6$. This result verifies the assertion that the acetate group is situated at $\mathrm{C} 6$, not $\mathrm{C} 5$, and that the structure of $\mathbb{Y}$ is indeed $\mathbf{3 . 2 8}$.

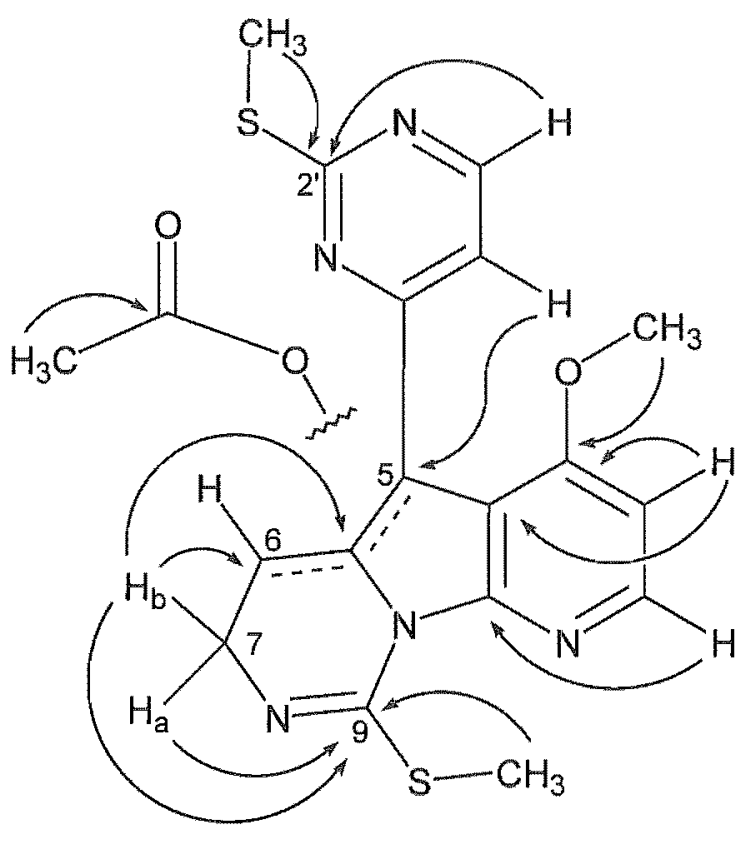

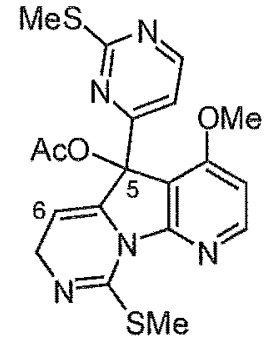

3.27

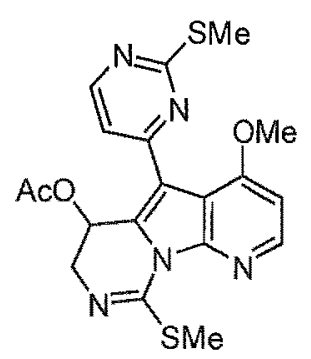

$3.28(Y)$

Figure 3.11 Important $\mathrm{HMBC}$ correlations for $\mathbf{Y}\left({ }^{\mathrm{n}} J_{\mathrm{CH}}=4\right.$ and $\left.9 \mathrm{~Hz}\right)$.

The isolation of 3.28 from the acetic acid/TES reaction may represent a significant advance in establishing the mechanism of the cyclisation, as it could be an intermediate in the formation of 1.63. It should be mentioned that this product was also observed in the TFA/TES reaction if that reaction was conducted at reflux for shorter periods. A possible mechanism for its formation is given in Figure 3.12. Initially, cyclisation to the pyridopyrrolopyrimidine cation 3.29 takes place. In Chapter Two, it was suggested that hydride reduction of such a species would occur at C5a. However, it would appear that, in the present case, the hydride attacks at $\mathrm{C} 7$ to produce the uncharged intermediate 3.30. At this stage, the acetate undergoes a rearrangement to give the isolated product 3.28. The driving force for the latter step is probably the formation of the aromatic indole nucleus, however, the combination of relief of steric strain at the central carbon and relief of bond angle strain by making the central 5-membered ring planar may also play a part. 
<smiles>COc1nccc(C(O)(c2ccnc(SC)n2)c2c(OC)ccnc2Cl)n1</smiles>

3.26
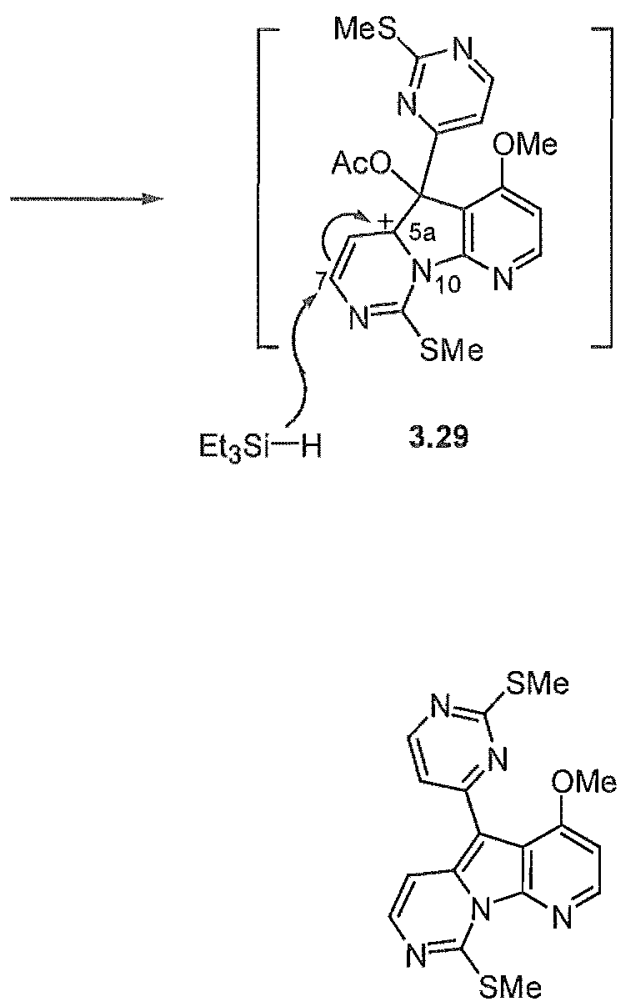

1.63

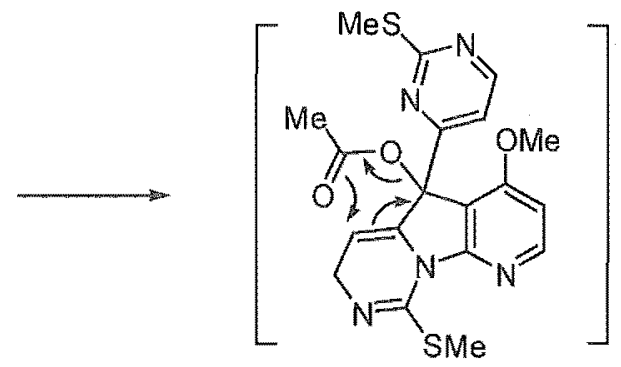

3.30<smiles>COc1ccnc2c1c(-c1ccnc(SC)n1)c1n2C(S(C)(C)C)=NCC1OC(C)=O</smiles>

3.28

Figure 3.12 Possible mechanism of formation of 3.28, and subsequent elimination of acetic acid to produce the variolin core structure $\mathbf{1 . 6 3}$.

It is not difficult to envisage that the elimination of acetic acid from 3.28 would give the variolin core structure 1.63. If this could be shown to be the case, this would provide persuasive evidence that 3.28 is, indeed, an intermediate in the formation of $\mathbf{1 . 6 3}$. To test this theory, 3.28 was heated in DCE with TFA to induce elimination of the acetate. However, this reaction resulted in a complex mixture in which, neither 3.28 , nor the variolin core 1.63 could be detected. This leaves the question of the role of 3.28 unanswered.

It may be that the predominant pathway involves hydride attack at C5a of 3.29, leading to the variolin core 1.63 , with a small amount of $\mathrm{C} 7$ attack leading to the side-product 3.28. Even if 3.28 is not involved in the formation of 1.63 , its isolation is still significant, in that it shows that cyclisation to the pyridopyrrolopyrimidine core can precede deoxygenation of the acetate. 


\subsubsection{Summary}

The TFA/TES hydrogenolysis pair was found to be an effective reagent system for the deoxygenation/cyclisation of triarylmethanol $\mathbf{1 . 6 5}$, producing the variolin core structure $\mathbf{1 . 6 3}$ in $47 \%$ yield. If, however, the alcohol is protected as the acetate, the reaction proceeds much more cleanly and the yield of $\mathbf{1 . 6 3}$ is increased to $75 \%$. A minor product of the reaction was identified, which lends some insights into the mechanism of cyclisation. However, further work is required in this regard, if a definitive mechanism is to be established. 


\subsection{Synthesis of Variolin $\mathbf{B}$}

Now that the variolin core $\mathbf{1 . 6 3}$ could be produced in good yield, the completion of the total synthesis of variolin B (1.17) requires substitution of the thiomethyl groups with amines and deprotection of the methoxy group. While the introduction of protected amines and their deprotection to free $\mathrm{NH}_{2}$ groups had already been accomplished for the deoxy-system (see Chapter Two), an efficient means of cleaving the methoxy group had to be found. In addition to selecting an appropriate reagent, the specific order of the remaining steps would be important. As $p$-methoxybenzyl groups would be used to protect the amines, methoxy deprotection on the bis-protected amine could result in mixtures of products, due to the presence of the two extra methoxy groups. For this reason, it was felt that methoxy deprotection would best be performed prior to amine substitution on structure 1.63.

\subsubsection{Preliminary Studies in Deprotection of the Methoxy Group}

\subsubsection{Boron Tribromide}

A particularly mild and effective method for the cleavage of ethers utilises boron tribromide. ${ }^{115}$ This agent complexes strongly to Lewis bases, such as the ethereal oxygen of a methyl aryl ether (Figure 3.13). Cleavage of the ether produces methyl bromide and a boron-phenolate complex which is protonated on acidic workup to give the phenol. It is recommended that an extra cquivalent of the reagent be added for every Lewis basic group in the substrate, as these can complex to the boron and thus remove it from solution.
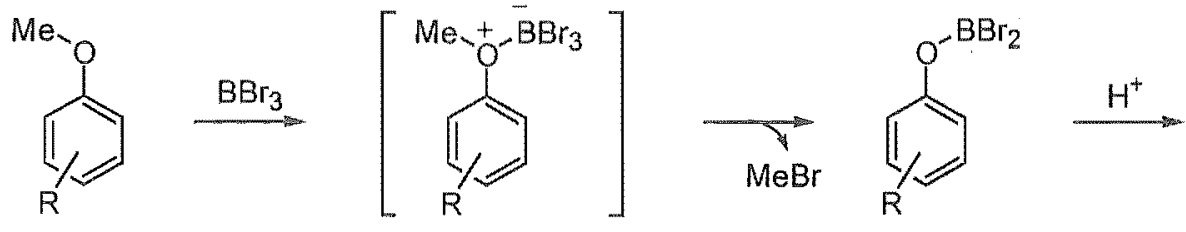<smiles>[R]c1ccc(O)cc1</smiles>

Figure 3.13 Deprotection of methyl aryl ethers by boron tribromide. 
A solution of 1.63 in $\mathrm{CH}_{2} \mathrm{Cl}_{2}$ was treated with boron tribromide (10 equivalents) at $-70{ }^{\circ} \mathrm{C}$, and allowed to warm to room temperature, producing an orange precipitate. However, TLC analysis indicated that only starting material was present. It is thought that the nitrogen atoms in the substrate were coordinating to the boron, causing the variolin-boron tribromide complex to precipitate out of solution. When more forcing conditions were applied (heating the heterogeneous mixture at $65{ }^{\circ} \mathrm{C}$ in DCE), several new products were formed. However, the bright orange precipitate would not dissolve in either $\mathrm{CH}_{2} \mathrm{Cl}_{2}$ or water. After filtering and washing the solid, a ${ }^{1} \mathrm{H}$ NMR spectrum was obtained in $\mathrm{CD}_{3} \mathrm{OD}$. A singlet at $\delta 4.13$ indicated that the methoxy group was still intact. Six aromatic protons were observed, as expected. Surprisingly, however, only one thiomethyl signal (integrating for three protons) could be seen. The ${ }^{13} \mathrm{C}$ NMR spectrum (in DMSO-d ${ }_{6}$ ) confirmed both the presence of the methoxy group, with a signal at $\delta 56.8$, and the loss of one of the thiomethyl groups (only one thiomethyl signal was seen at $\delta 13.7)$.

When the ${ }^{1} \mathrm{H}$ NMR spectrum was obtained in DMSO- $\mathrm{d}_{6}$, a broad signal at $\delta 13.0$ was seen which was not present in the $\mathrm{CD}_{3} \mathrm{OD}$ spectrum. Such a signal is indicative of a hydrogen bonded exchangeable proton. Electrospray MS revealed that the molecular formula of this product was $\mathrm{C}_{16} \mathrm{H}_{13} \mathrm{~N}_{5} \mathrm{OS}_{2}$. Thus, it would appear that although the molecule contains two sulfurs, only one of these belongs to a thiomethyl group. It seemed that the other thiomethyl group had been cleaved to give a thiol, in preference to deprotection of the methoxy group on the pyridine ring. Using 2D NMR spectroscopy, the position of the thiol was shown to be at $\mathrm{C} 9$, as indicated in structure 3.31. This regiochemistry is to be expected, based on the chemical shift of the thiol ${ }^{1} \mathrm{H}$ signal, as it allows hydrogen bonding of the thiol proton to the pyridine nitrogen (N1). This type of hydrogen bonding was observed for the amine 2.70 (Chapter Two), and of course, for deoxyvariolin $\mathrm{B}(\mathbf{1 . 5 0})$ and variolin $\mathrm{B}(\mathbf{1 . 1 7}){ }^{32 \mathrm{a}}$ Important correlations observed in the CIGAR experiment are shown in Figure 3.14 . 


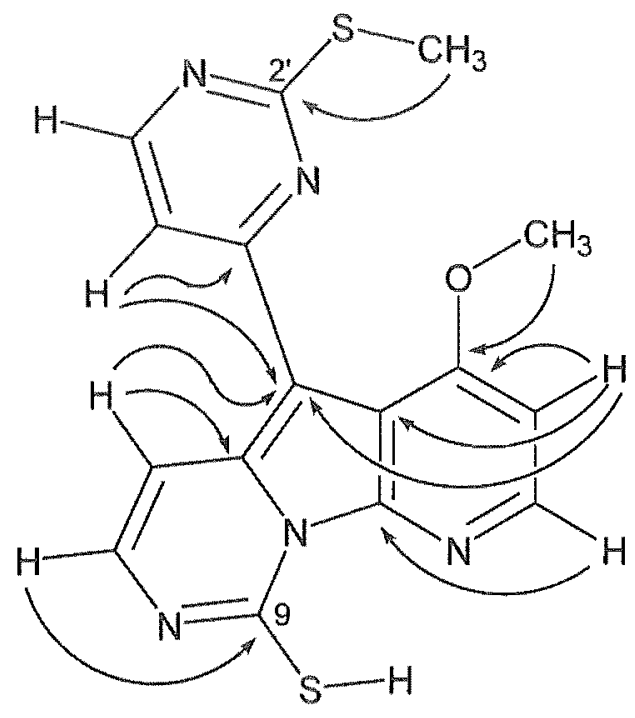

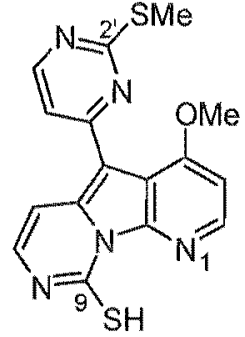

3.31

Figure 3.14 Important CIGAR correlations for 3.31.

Presumably, $\mathbf{3 . 3 1}$ arises by a similar mechanism to that expected for methoxy deprotection. As the 9-SMe group appeared to be more reactive than the methoxy group when using boron tribromide, an altemative reagent was required. Based on the experience with boron tribromide, it was decided to avoid Lewis acid reagents, as insoluble complexes would always be a possibility if such reagents were applied to the variolin poly-N-heterocyclic system.

\subsubsection{Sodium Ethanethiolate}

Sodium ethanethiolate is a commonly used reagent for the deprotection of methyl aryl ethers. ${ }^{116}$ Reactions are usually carried out in an aprotic dipolar solvent such as DMF, usually at refluxalthough in exceptional cases, reactions proceed at room temperature. Deprotection occurs by $\mathrm{S}_{\mathrm{N}} 2$ attack at the methoxy group to produce a phenolate anion, which is protonated on workup to give the phenol. Such a mechanism would be well-suited to the present case, as the resulting pyridinol anion would be stabilised by delocalisation onto the pyridine nitrogen (N1).

The reaction was initially conducted at $95{ }^{\circ} \mathrm{C}$ with an excess of sodium ethanethiolate (20 equivalents) and monitored by TLC. After only $0.5 \mathrm{~h}$, it was observed that the starting material 1.63 had been consumed and several new products had been generated. ${ }^{1} \mathrm{H}$ NMR spectroscopy 
of the crude mixture revealed at least three products, all of which appeared to have undergone clean methoxy-deprotection. However, varying degrees of nucleophilic substitution of the SMe groups (both, one or none) by sodium ethanethiolate had also taken place. Despite the mixtures of products, these results were encouraging. If substitution of the thiomethyl groups could be avoided, this reagent would serve our purposes well.

In an attempt to preclude SMe substitution, the reaction was repeated, but at room temperature. After $1 \mathrm{~h}$, a ${ }^{1} \mathrm{H}$ NMR spectrum of a small aliquot was obtained, which indicated that a significant amount of methoxy deprotection had occurred $(\sim 20 \%)$. However, one of the SMe groups had been almost completely substituted with SEt. Based on the results of selective amine substitution discussed in Chapter Two, it is presumed that substitution of the 9-SMe group had taken place. It would appear that this reaction proceeds at a much faster rate than methoxy deprotection. To avoid this complication, it was decided to leave the methoxy deprotection until after the SMe groups had been replaced with the protected amines. These groups would be far less readily substituted by sodium ethanethiolate. While they do contain potentially reactive methoxy groups, it seemed that cleavage of the pyridyl methyl ether was an especially facile process. The resulting phenolate would be a much better leaving group than an ordinary phenolate, and thus it was felt that selective cleavage may be possible in the presence of the $p$ methoxybenzylamines.

\subsubsection{Oxidation/Nucleophilic Substitution of Thiomethyl Groups}

Thus, core structure 1.63 was subjected to oxidation with $m$-CPBA using the conditions that were used for the oxidation of the thiomethyl groups of the deoxy-variolin core 2.1. As expected, the major product was the bis-sulfoxide $\mathbf{3 . 3 2}$. When the reaction was repeated at lower temperature $\left(-40^{\circ} \mathrm{C}\right)$ using 2 equivalents of oxidising agent, the bis-sulfoxide was produced much more cleanly than before. In the ${ }^{1} \mathrm{H}$ NMR spectrum of the crude product, a very small peak at $\delta 2.69$ indicated that oxidation of one of the SMe groups was not quite complete. An equally small peak at $\delta 3.40$ suggested a small degree of oxidation to the sulfone. However, the bis-sulfoxide comprised $>90 \%$ of the mixture (judging by ${ }^{1} \mathrm{H}$ NMR spectroscopy). As with the deoxy-equivalent $\mathbf{2 . 6 9}$, the product exists as a 1:1 mixture of diastereomers, due to the chirality of the two sulfoxides. The signals for the SOMe protons appeared at $\delta 3.02$ and 3.19 which, as 
explained in Chapter Two, is typical for this type of system. The aromatic signals shifted downfield from those of the starting material, in a manner analogous to that observed in the deoxy-series.

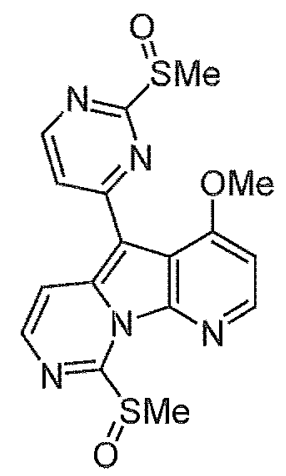

3.32

While the crude mixture should undergo amine substitution to give just one product, purification was attempted for the purpose of completely characterising the bis-sulfoxide 3.32. Silica flash chromatography of this compound requires methanol/ $/ \mathrm{CH}_{2} \mathrm{Cl}_{2}$ mixtures due to its high polarity. However, ${ }^{1} \mathrm{H}$ NMR analysis of the "purified" product revealed a new compound, present in $\sim 30 \%$, along with the bis-sulfoxide. The most notable features in the spectrum of the new product were that one SOMe peak was absent, while a new peak at $\delta 4.39$, integrating for three protons, had appeared. As this product was not observed in the ${ }^{1} \mathrm{H}$ NMR spectrum of the crude mixture, it must result from decomposition of the bis-sulfoxide on silica. Structure 3.33 is proposed for this product. The methoxy group must come from the eluant used in the chromatography ( $8 \%$ methanol/ $\mathrm{CH}_{2} \mathrm{Cl}_{2}$ ). This facile substitution, which is possibly catalysed by the acidic silica, highlights the highly electron-deficient nature of the fused pyrimidine ring, especially upon oxidation of sulfur to the sulfoxide.

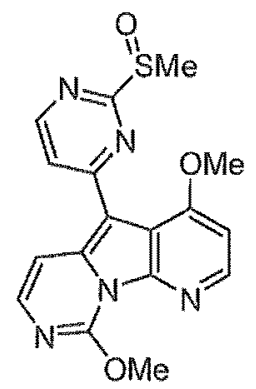


Despite trying a variety of other solvent systems on both silica and alumina TLC plates (including ethyl acetate, THF and $\mathrm{CH}_{3} \mathrm{CN}$ as the polar components), none could be found that would allow the polar bis-sulfoxide to move appreciably off the baseline. Thus, only ${ }^{1} \mathrm{H}$ NMR data for $\mathbf{3 . 3 2}$ is included in the Experimental section of this thesis. It was decided to continue with the amine substitutions on the crude oxidised mixture.

Therefore, the crude oxidised mixture, consisting mainly of 3.32 , was heated at $85^{\circ} \mathrm{C}$ in neat $p$ methoxybenzylamine. After $15 \mathrm{~h}$, complete substitution of all $\mathrm{SO}_{\mathrm{x}} \mathrm{Me}$ groups (where $\mathrm{x}=0,1,2$ ) had taken place. The mixture was, without workup, purified by flash chromatography to give the desired product, bis-protected amine 3.34, in 78\% yield over the two steps (Scheme 3.13). The identity of the product was clear, based on comparison of its ${ }^{1} \mathrm{H}$ NMR spectrum with that of the deoxy-equivalent 2.70. In particular, the two $\mathrm{NH}$ signals were easily recognised, with the 9-NH signal appearing as a broadened triplet at $\delta 10.39$ due to hydrogen bonding with the pyridine nitrogen (N1), and the 2 - $\mathrm{NH}$ signal appearing as a broad signal at $\delta 5.51$. The COSY experiment showed that the NH signals are coupled to separate doublets, which correspond to the methylene protons of the two p-methoxybenzyl groups. All other spectroscopic data are in agreement with the structure 3.34 .

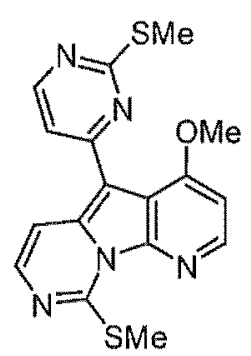

1.63

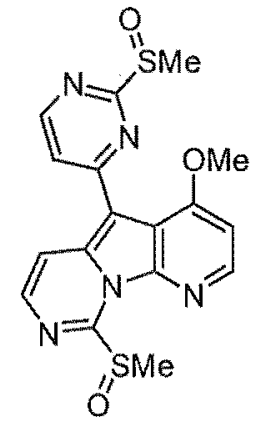

3.32

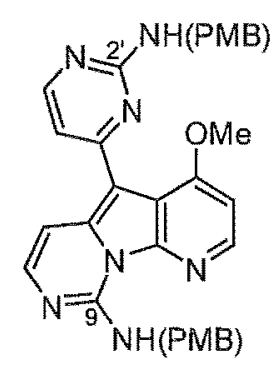

3.34

Scheme 3.13 Reagents and yields: (i) $m$-CPBA (2 equiv) $/ \mathrm{CHCl}_{3} /-40{ }^{\circ} \mathrm{C}$; (ii) $p$-methoxybenzylamine (11 equiv) $/ 85^{\circ} \mathrm{C}$ (78\% from 1.63$)$. 


\subsubsection{Deprotection of the Methoxy Group}

As the thiomethyl groups were no longer of consequence, methoxy deprotection was reattempted with sodium ethanethiolate. When heated at $95{ }^{\circ} \mathrm{C}$, a new product formed rapidly (judging by TLC analysis). ${ }^{1} \mathrm{H}$ NMR analysis of the mixture revealed clean deprotection of the pyridine methoxy group, while those of the PMB groups remained intact. Even when the mixture was heated at $130^{\circ} \mathrm{C}$ for $12 \mathrm{~h}$ with 20 equivalents of sodium ethanethiolate, the PMB methoxy groups were untouched. After optimisation of the conditions, the pyridine methoxy group was found to be readily cleaved on heating at $55^{\circ} \mathrm{C}$ for $7.5 \mathrm{~h}$ to give the pyridinol 3.35 as the only variolin-type product. Flash chromatography on silica allowed the isolation of $\mathbf{3 . 3 5}$ in pure form in 95\% yield (Scheme 3.14).

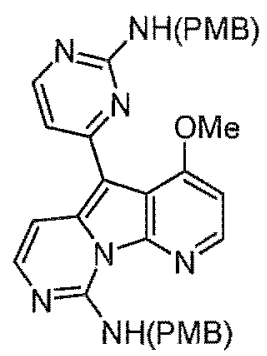

3.34

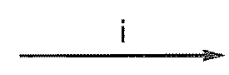

3.35

Scheme 3.14 Reagents and yields: (i) NaSEt (10 equiv)/DMF/55 ${ }^{\circ} \mathrm{C}(95 \%)$.

The most distinctive feature in the ${ }^{1} \mathrm{H}$ NMR spectrum of 3.35 is a broad signal at $\delta 15.7$. This very deshielded signal was assigned to the hydroxyl proton and its chemical shift probably reflects internal hydrogen bonding to $\mathrm{N}^{\prime}$ ' of the pendant pyrimidine ring. Interestingly, the most significant change in the aromatic region involves $\mathrm{H} 6$ and H7. The signals for these protons were at $\delta 7.29$ and 7.43 respectively in the starting material 3.34. Upon deprotection of the ether, the signal for $\mathrm{H} 6$ has moved further upfield to $\delta 7.03$, while the signal for $\mathrm{H} 7$ has moved further downfield to $\delta 7.68$. These shifts were rather unexpected, as the putative hydrogen bond between $\mathrm{N}^{\prime}$ and the $\mathrm{OH}$ proton is expected to bring the pendant pyrimidine ring more into the 
plane of the pyridopyrrolopyrimidine ring system. An intense NOE correlation between $\mathrm{H}^{\prime}$ and H6 shows that these protons do lie quite close togther in space. It was thought that the aromatic ring current of the pendant pyrimidine ring would cause deshielding of $\mathrm{H6}$, and the signal would be expected to be seen further downfield than in $\mathbf{3 . 3 4}$.

An argument that may account for the observed values relies on electronic effects rather than conformational effects. It is thought that once the free hydroxyl group is uncovered, the hydrogen bond between $9-\mathrm{N} H$ and the pyridine nitrogen is strengthened. This assertion is supported by the fact that the ${ }^{1} \mathrm{H}$ signal for 9-NH appears at $\delta 10.9$ for the pyridinol 3.35, compared to $\delta 10.4$ for the precursor, 3.34. This would result in a redistribution of the lone-pair of electrons on 9-N, with more electron density in the fused pyrimidine ring. This would particularly affect the signal for H6, causing it to move upfield.

\subsubsection{Removal of the p-Methoxybenzyl Protecting Groups}

Dissolution of $\mathbf{3 . 3 5}$ in triflic acid immediately produced a blood-red solution, which was left at room temperature for $17 \mathrm{~h}$. Upon careful neutralisation of the mixture with aqueous ammonia, a bright yellow precipitate developed. ${ }^{1} \mathrm{H}$ NMR analysis of the product indicated that clean deprotection of both amines had taken place, to give the target compound variolin $B(1.17$, Scheme 3.15).

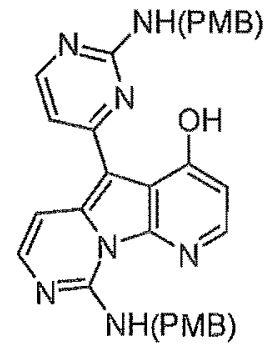

3.35

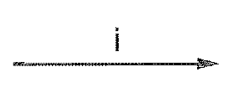

1.17

Scheme 3.15 Reagents and yields: (i) triflic acid/rt (80\%). 
The purification of the natural product was made difficult by its insolubility in most common solvents, other than DMSO. ${ }^{32 a}$ However, if it is converted to the trifluoroacetate salt, its solubility in methanol is increased. In this form, flash chromatography could be carried out on reversed phase silica to remove the PMB impurities, giving variolin $\mathrm{B}$ as its trifluoroacetate salt. This product was neutralised with aqueous ammonia/methanol and the ammonium trifluoroacetate, thus produced, was removed by high-vacuum sublimation $\left(35^{\circ} \mathrm{C}, 0.03 \mathrm{~mm} \mathrm{Hg}\right)$. Variolin B was obtained as a brown-orange solid in $80 \%$ yield.

In the original paper reporting the isolation of variolin $B, N M R$ data was reported for both the TFA salt and the free base. However, no clear definition was given as to what the "salt" is, other than stating that "Most NMR spectral data were obtained in a mixture of deuterated DMSO and TFA (salt) or DMSO alone (free base)..." ${ }^{32 a}$ In addition, it was found that chemical shifts of the salt were variable depending on the exact amount of TFA present. To avoid this ambiguity, all spectral data for synthetic variolin B were obtained as the free base.

The ${ }^{1} \mathrm{H}$ NMR spectrum of synthetic variolin B (Figure 3.15) is gratifyingly simple. Six doublets falling between $\delta 6.8$ and 8.3 represent the aromatic ring protons. A singlet integrating for two protons is due to the $2^{\prime}-\mathrm{NH}_{2}$ group, while the $9-\mathrm{NH}_{2}$ protons give rise to separate broadened signals due to restricted rotation about the $\mathrm{C} 9-\mathrm{NH}_{2}$ bond, as explained in Chapter One. The only other signal (at $\delta 16.05$ ) is from the hydroxyl proton.

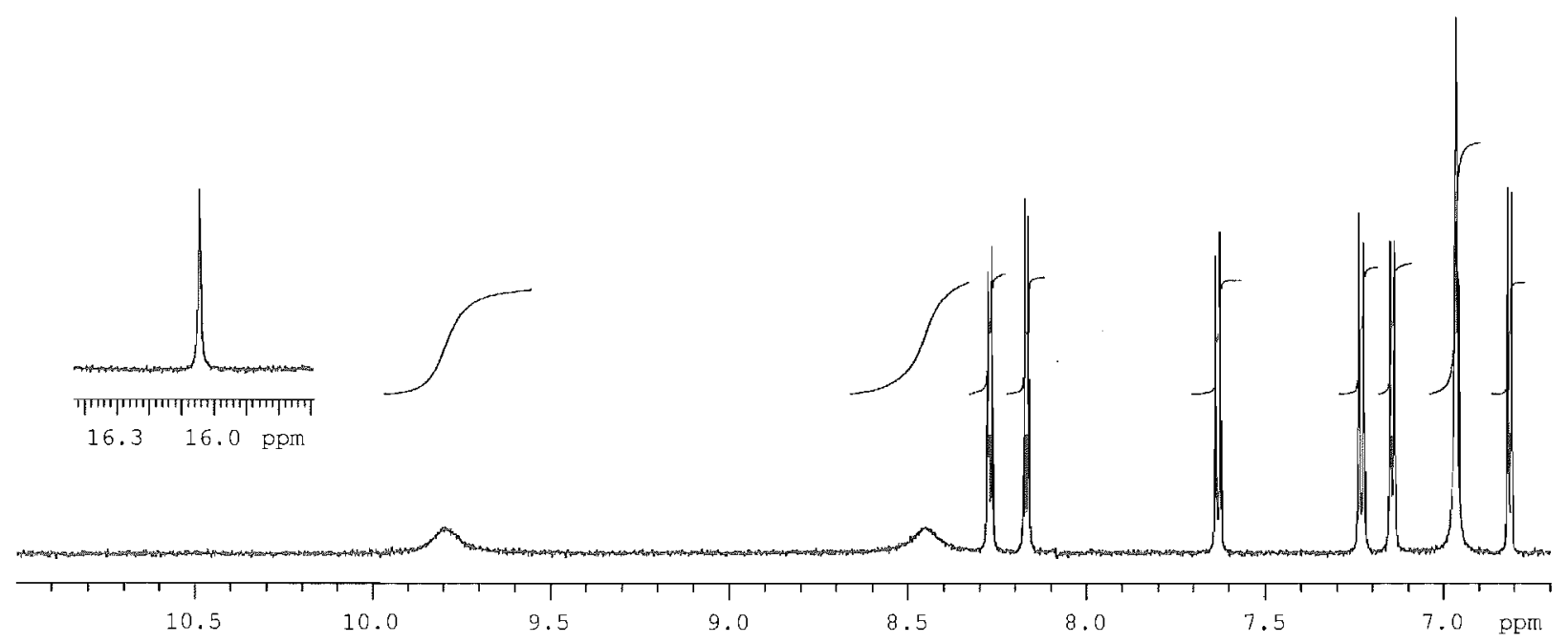

Figure 3.15 $500 \mathrm{MHz}{ }^{\mathrm{l}} \mathrm{H}$ NMR spectrum of synthetic variolin B (1.17) in DMSO-d 6 
As can be seen from Table 3.1, both the ${ }^{1} \mathrm{H}$ and ${ }^{13} \mathrm{C}$ chemical shift values agree very well with those of natural variolin B. A slight discrepancy can be seen regarding the assignments of $\mathrm{C}^{\prime}$ and C4'. The CIGAR experiment (on the synthetic material) showed a strong correlation from H6' to the carbon at $\delta 161.4$, which confirms that this peak belongs to C2'. Both H5' and H6' showed correlations to the carbon at $\delta 158.3$, which is to be expected if this peak belongs to C4'. Upon personal communication with the authors of the original paper, it was conceded that these ${ }^{13} \mathrm{C}$ signals may have been mis-assigned.

\begin{tabular}{c|cc|cc}
\hline \multirow{2}{*}{ position } & \multicolumn{2}{|c|}{${ }^{1} \mathrm{H}$} & \multicolumn{2}{c}{${ }^{13} \mathrm{C}$} \\
\hline 2 & synthetic $^{\mathrm{a}}$ & natural $^{\mathrm{b}}$ & synthetic $^{\mathrm{c}}$ & natural \\
\hline 3 & 6.17 & 8.14 & 143.1 & 143.2 \\
4 & & 6.78 & 107.5 & 107.6 \\
$4 \mathrm{a}$ & & & 159.8 & 159.9 \\
5 & & & 111.2 & 111.2 \\
$5 \mathrm{a}$ & & & 99.6 & 99.6 \\
6 & 7.23 & 7.19 & 137.1 & 137.1 \\
7 & 7.63 & 7.61 & 144.6 & 144.6 \\
9 & & & 150.3 & 150.3 \\
$10 \mathrm{a}$ & & & 144.9 & 145.0 \\
$2^{\prime}$ & & & 161.4 & 158.4 \\
$44^{\prime}$ & & & 158.3 & 161.5 \\
$5^{\prime}$ & 7.14 & 7.11 & 106.0 & 106.1 \\
$66^{\prime}$ & 8.27 & 8.25 & 160.0 & 160.1 \\
$9-\mathrm{NH}_{2}$ & 8.45 & 8.5 & & \\
$9-\mathrm{NH}_{2}$ & 9.79 & 9.8 & & \\
$2^{\prime}-\mathrm{NH}_{2}$ & 6.97 & 6.95 & & \\
$4-\mathrm{OH}^{\prime}$ & 16.05 & 16.0 & & \\
\hline
\end{tabular}

Table 3.1 Comparison of ${ }^{1} \mathrm{H}$ and ${ }^{13} \mathrm{C}$ NMR chemical shifts for synthetic and natural variolin $\mathrm{B}^{32 \mathrm{a}}$

${ }^{a}$ Recorded in DMSO- $\mathrm{d}_{6}$ at $500 \mathrm{MHz} .{ }^{\mathrm{b}}$ These values have been adjusted by $-0.12 \mathrm{ppm}$ from those in the original paper, so that the solvent reference peak $(\delta 2.62)$ corresponds to the present system $(\delta 2.50 \mathrm{ppm})$. ${ }^{\mathrm{c}}$ Recorded in DMSO-d $\mathrm{d}_{6}$ at $75 \mathrm{MHz}$. 
As shown in Table 3.2, the melting point and IR data of synthetic variolin B do not correspond well to those of the natural material. The reason for these differences is not known-it may be that the data of the natural material were obtained from the TFA salt. However, the TFA salt of synthetic variolin B (obtained by crystallisation from an aqueous TFA solution) showed no indication of any decomposition at, or near, $45^{\circ} \mathrm{C}$.

\begin{tabular}{c|l|l}
\hline & \multicolumn{1}{|c|}{ synthetic (free base) } & \multicolumn{1}{c}{ natural (salt?) } \\
\hline MS & ESMS: $294.1096\left(\mathrm{MH}^{+}\right)$ & HREIMS: $293.102\left(\mathrm{M}^{+}\right)$ \\
Mp $\left({ }^{\circ} \mathrm{C}\right)$ & $\sim 320(\mathrm{dec})$ & $45(\mathrm{dec})$ \\
$\operatorname{IR}\left(\mathrm{cm}^{-1}\right)$ & $3085,1670,1572,1477,1458$, & $3100,1684,1614,1540,1497,1310$, \\
& 1298 & 1188,1135 \\
\hline
\end{tabular}

Table 3.2 Comparison of spectroscopic data for synthetic and natural variolin $B .^{32 a}$

As a sample of natural variolin B in the salt form was available, ${ }^{1} \mathrm{H}$ NMR spectra of the TFA salts of synthetic and natural variolin $B$ were obtained side by side under the same conditions. It should be noted that the texture of the natural material was that of a paste, whereas synthetic variolin B was obtained as a free-flowing powder, or in micro-crystalline form when recrystallised from water/TFA.

The ${ }^{1} \mathrm{H}$ NMR spectra of the two samples are shown in Figure 3.16a and $\mathbf{b}$. It is clear that the chemical shifts in these spectra differ somewhat - this is presumably due to different amounts of TFA being present in each sample. To prove that the synthetic and natural samples are, indeed, one and the same compound, the two NMR solutions were thoroughly mixed together and the ${ }^{1} \mathrm{H}$ NMR spectrum was re-run. The result is shown in Figure 3.16c. Only one compound can be seen, making it absolutely clear that the synthetic material is, indeed, variolin B (1.17). 

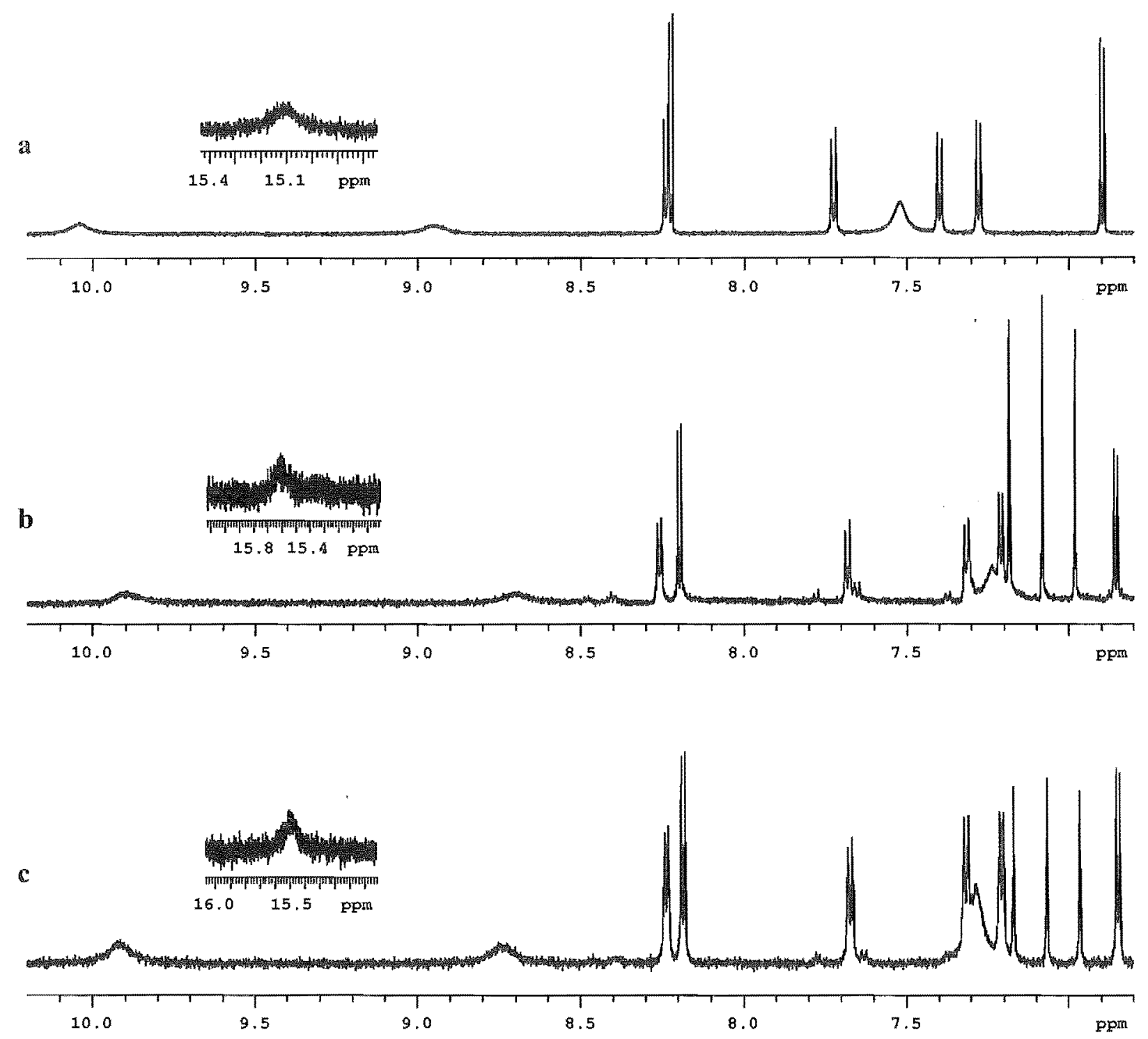

Figure 3.16 (a) $500 \mathrm{MHz}^{1} \mathrm{H}$ NMR spectrum of synthetic variolin B (TFA salt); (b) $500 \mathrm{MHz}$ ${ }^{1} \mathrm{H}$ NMR spectrum of natural variolin $\mathrm{B}$ (TFA salt); (c) $500 \mathrm{MHz}{ }^{1} \mathrm{H}$ NMR spectrum of a mixture of synthetic and natural variolin $B$ (TFA salt).

\footnotetext{
"The three peaks between $\delta 6.9$ and 7.2 had also been observed in synthetic samples of variolin B early onsometimes in such great proportions that they overwhelmed all other peaks in the spectrum. They appear to be a consequence of incomplete neutralisation of the TFA salt with aqueous ammonia solution. The three peaks arise from the protons of $\mathrm{NH}_{4}^{+}$. In strongly acidic solutions, the quadrupolar coupling of these protons to ${ }^{14} \mathrm{~N}$ can be observed, giving rise to a 1:1:1 triplet (Tony Carroll, personal communication).
} 


\subsection{Summary}

The target compound variolin B (1.17) has been synthesised in just eight steps from commercially available 4-chloro-2-(methylsulfanyl)pyrimidine (2.13, Scheme 3.16). Some modifications to the strategy described in Chapter Two were required for the synthesis of triarylmethanol 1.65 , as addition of the pyrimidinyl lithio-species 1.66 to acid chloride 1.67 was too slow at $-95^{\circ} \mathrm{C}$. However, the more stable lithiated pyridine 3.16 and bipyrimidinyl ketone 3.15 were found to react smoothly at $-78{ }^{\circ} \mathrm{C}$ to give the desired triarylmethanol 1.65 in $76 \%$ yield.

After careful optimisation of the cyclisation reaction conditions, the variolin core structure $\mathbf{1 . 6 3}$ could be produced in $47 \%$ yield from 1.65 . However, if the triarylmethylacetate 3.26 was used as an alternative to $\mathbf{1 . 6 5}$, the cyclisation step proceeded much more cleanly and efficiently to give 1.63 in $75 \%$ yield. Further modification of this compound involved the substitution of the thiomethyl groups for protected amines and subsequent deprotection of both the amines and the methoxy group. This resulted in the first total synthesis of variolin B, in an overall yield of $13 \%$ for the eight steps. ${ }^{39 b}$

This route is attractive due to its brevity and efficiency. It compares favourably to the total synthesis reported recently by Molina, Fresneda and coworkers, which required 16 steps and proceeded in an overall yield of $1.2 \% .{ }^{36}$ Importantly, our route will provide a supply of synthetic variolin B, which will allow further exploration of its biological properties and potential as a therapeutic agent. 
<smiles>[X]c1ccnc(S(C)(=O)=O)n1</smiles><smiles>C#CC</smiles>

i

$2.13 \mathrm{X}=\mathrm{Cl}$

2.12 $x=1$

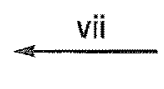

$\mathrm{NH}(\mathrm{PMB})$

3.35

1.17

variolin $B$<smiles>CCCCCCCCC</smiles><smiles>CSc1nccc(C(=O)c2ccnc(SC)n2)n1</smiles>

3.15
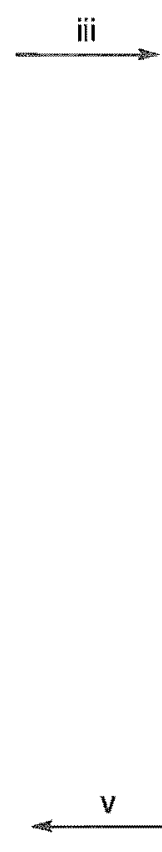<smiles>[X]c1nccc(-c2c3c(OC)ccnc3n3c([X])nccc23)n1</smiles>

vi $\left[\begin{array}{l}3.32 X=\text { SOMe } \\ 3.34 X=N H(P M B)\end{array}\right.$<smiles>COc1ccnc(Cl)c1C(OC)(c1ccnc(SC)n1)c1ccnc(SC)n1</smiles>

3.26<smiles>COc1ccnc2c1c(-c1ccnc(C)n1)c1ccnc(SC)n12</smiles>

1.63<smiles>COc1ccnc(Cl)c1</smiles>

Scheme 3.16 Reagents and yields: (i) $55 \%$ aq HI/rt (79\%); (ii) $n$-BuLi (1 equiv)/THF/-95 ${ }^{\circ} \mathrm{C}, 30 \mathrm{~min}$ then diethyl carbonate $\left(0.5\right.$ equiv) $/ \mathrm{THF} / 95{ }^{\circ} \mathrm{C}$ to $\mathrm{rt}(53 \%)$; (iii) $3.8: n$-BuLi (1 equiv)/THF $-90^{\circ} \mathrm{C}$ to $-78^{\circ} \mathrm{C}, 1 \mathrm{~h}$ then 3.15 ( 1 equiv) $/ \mathrm{THF} / .90^{\circ} \mathrm{C}$ to $-78{ }^{\circ} \mathrm{C}, 3 \mathrm{~h}$ then $\mathrm{AcCl}$ ( 4 equiv) $/-78{ }^{\circ} \mathrm{C}$ to $\mathrm{rt}\left(68 \%\right.$ ); (iv) TFA (2 equiv) $/ \mathrm{TES}$ ( 8 equiv) $/ \mathrm{DCE} / 80^{\circ} \mathrm{C} / \mathrm{sealed}$ tube $(75 \%) ;(\mathrm{v}) m$-CPBA (2 equiv) $/ \mathrm{CHCl}_{3} /-40{ }^{\circ} \mathrm{C}$; (vi) $p$-methoxybenzylamine $(10$ equiv) $/ 85^{\circ} \mathrm{C}(78 \%$ from 1.63$)$; (vii) $\mathrm{NaSEt}\left(10\right.$ equiv)/DMF $/ 55^{\circ} \mathrm{C}$ (95\%); (viii) triflic acid/rt $(80 \%)$. 


\section{CHAPTER FOUR}

Structure-Activity Relationships and Synthesis of Analogues 


\subsection{Introduction}

It was stated in the Introduction of this dissertation that a total synthesis of variolin B should not only be direct and efficient, but also be amenable to the production of a wide variety of analogues. Analogue synthesis is an important part of drug development, as it can allow the fine-tuning of important pharmacological parameters. These include potency, toxicity, bioavailablity, solubility in cellular fluids, biostability and site selectivity of the drug. Inadequate performance in any one of these areas could rule the drug out as a medicinal agent. With a range of analogues, however, these obstacles may be overcome if a candidate can be found which meets all the necessary requirements.

Analogues are also important in structure-activity relationship (SAR) studies. The structure of the drug can be altered in degrees by chemical transformation and the biological activity of each analogue can be determined. Correlation of the observed activity with chemical structure leads to SARs, thereby allowing the determination of the structural elements of the drug that are essential to its biological properties. This may eventually lead to a simpler target which is more easily obtainable, while retaining a similar degree of biological activity to the natural product. In addition, SARs may provide insights into the mode of action of the drug. For example, the essential components may be required for some intermolecular interaction with a macromolecule, such as an enzyme or other protein, the result of which will modulate its activity. Such knowledge will allow further development of the drug by modifying its structure so as to maximise these interactions.

\subsubsection{Definitions}

While the term "drug" strictly refers to a chemical which has been approved for medicinal use, it is used here of any compound made for the purpose of investigating its biological activity. The term "biological activity" is often used vaguely to describe the property of any chemical which may cause an alteration of a cell's normal state. In this Chapter, it refers specifically to performance in the P388 assay. This assay measures the growth inhibition of a murine leukaemia cell line in the presence of a known concentration of the drug. The parameter derived from the assay, $\mathrm{IC}_{50}$, represents the concentration of drug required to reduce the $\mathrm{P} 388$ cell growth 
by $50 \%$ comparative to control cells. $\mathrm{IC}_{50}$ is measured in $\mathrm{ng} / \mathrm{mL}$; thus, a lower value corresponds to a higher degree of potency. The reported $\mathrm{IC}_{50}$ value for natural variolin $\mathrm{B}$ is 210 ng/mL. ${ }^{32 a}$ Thus, compounds tested in the P388 assay in this thesis are compared to variolin $B$ and can be said to have increased or decreased activity, as compared to the natural product. Compounds with an $\mathrm{IC}_{50}>20,000 \mathrm{ng} / \mathrm{mL}$ are classed in this Chapter as being inactive. This value has been somewhat arbitrarily chosen, although it corresponds to a 100-fold loss of activity compared with variolin $B$.

\subsubsection{Biological Data of Synthetic Variolin B}

Before undertaking a project in the synthesis of variolin analogues, it was of crucial importance to first determine the biological properties of synthetic variolin B. With any biologically active extract, there exists the possibility that the activity of the natural material is not due to the characterised product (in this case, variolin B) but rather an unknown trace impurity. Therefore, synthetic variolin B was tested in the P388 assay, along with a sample of natural variolin B for comparison.

Pleasingly, synthetic variolin $\mathrm{B}$ was found to have an $\mathrm{IC}_{50}=189 \mathrm{ng} / \mathrm{mL}$, which is essentially equal to that reported for the natural material $\left(\mathrm{IC}_{50}=210 \mathrm{ng} / \mathrm{mL}\right){ }^{32 \mathrm{a}}$ It should be pointed out that the P388 assay is only accurate to $\sim 50 \%$. Thus, the difference between these two values is trivial-significant differences are measured in orders of magnitude. In the present assay, the naturally derived variolin $\mathrm{B}$ showed significantly lower activity, with an $\mathrm{IC}_{50}=465 \mathrm{ng} / \mathrm{mL}$. It may be that this sample has degraded over the years. Also of relevance is that this sample of natural variolin B was stated to be the TFA salt form. As has been discussed, there is no clear definition as to what this means. It may be that up to $50 \%$ of the sample is actually TFA, which would explain its higher $\mathrm{IC}_{50}$. This aside, the key result is that synthetic variolin $B$ shows at least equal potency to that of the natural material. This result rules out this possibility that the bioactivity originates from an unidentified contaminant of the natural material.

Further testing on synthetic variolin B has been carried out by Pharma Mar (who have the commercial rights to this synthesis). Preliminary results have been encouraging, with variolin $B$ displaying anti-proliferative activity against a range of human tumour cell lines. This has resulted in variolin $\mathrm{B}$ being entered into Safety Trials. 


\subsection{Structure-Activity Relationships in the Variolins}

\subsubsection{Introduction}

Throughout the course of Chapters Two and Three, it was seen that a limited range of nucleophiles were introduced into either of the variolin core structures 2.1 or 1.63 -sometimes deliberately and sometimes unintentionally. As substitution products were obtained using amines (primary and secondary), methanol and thiolate as nucleophiles, it appears that this type of substitution is quite general in the variolin system. This type of reactivity offers a powerful means of rapidly producing a range of analogues. It was also noted in Chapter Two that selective substitution of the 9-SMe group can be conveniently achieved by simply heating $\mathbf{2 . 1}$ with the nucleophile in a suitable solvent. This will allow for the production of an even broader spectrum of analogues. Substitution of the 9-SMe group with an amine nucleophile, for example, followed by $S$-oxidation of the 2 '-SMe group and subsequent replacement with a different nucleophile should increase the variety of analogues that can be produced. It would also allow a comparison of the importance of these two sites for biological activity. Alternatively, if identical substituents are required at both $\mathrm{C} 9$ and $\mathrm{C} 2$ ', oxidation of 2.1 or 1.63 followed by double substitution would provide the desired compound. This method was used in the syntheses of both deoxyvariolin B (1.50) and variolin B (1.17). Thus, the thiomethylcontaining structures $\mathbf{2 . 1}$ and $\mathbf{1 . 6 3}$ are key compounds for the synthesis of analogues of the variolins.

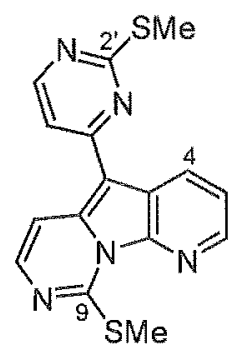

2.1

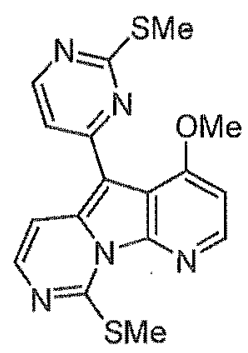

1.63

The oxygen at $\mathrm{C} 4$ of 1.63 also offers a convenient handle for increasing the diversity of analogues that can be produced. It has been shown that deprotection of the methoxy group can 
be readily achieved using sodium ethanethiolate to give the free hydroxyl group. A variety of groups could be appended to this oxygen to provide a new set of analogues, which would be examined for biological activity.

Thus, the three points of variation available are a) the substituent at C9 (group X), b) the substituent at $\mathrm{C}^{\prime}$ (group Y) and c) the substituent at C4 (group Z) (Figure 4.1). As the intermediates in Chapters Two and Three vary in these aspects, some of these were tested for biological activity. In addition, a range of analogues were produced to provide further variation of groups X, Y and Z.

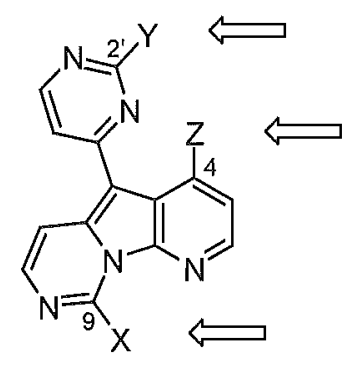

Figure 4.1 Points of variation available in the variolins.

\subsubsection{Variation in Group $\mathbb{Z}$}

\subsubsection{Synthesis}

Comparison of the biological properties of deoxyvariolin $B(\mathbb{1 . 5 0})(Z=H)$ and variolin $B(\mathbb{1 . 1 7})$ $(\mathrm{Z}=\mathrm{OH})$ would provide some insight into the importance of group $\mathrm{Z}$. A third variant where $\mathrm{Z}=\mathrm{OMe}$ should be readily accessible by removal of the PMB groups of $\mathbf{3 . 3 4}$, without prior deprotection of the methoxy group. To this end, $\mathbf{3 . 3 4}$ was dissolved in neat triflic acid and left at room temperature to give $O$-methylvariolin B (4.1) (Scheme 4.1). After neutralisation with aqueous ammonia solution, the ammonium triflate thus produced was removed by adsorbing the mixture onto a small $\mathrm{C}_{18}$ cartridge and eluting with $30 \% \mathrm{MeOH} /$ water. The yellow product was collected in a separate fraction upon elution with $25 \% \mathrm{MeOH} / \mathrm{CH}_{2} \mathrm{Cl}_{2}+0.2 \%$ TFA. While silica 
TLC of this partially purified product indicated that good separation should be achievable, when flash chromatography was carried out on silica, the yellow colour smeared down the length of the column. Addition of $0.5 \%$ triethylamine to the eluant $\left(10 \% \mathrm{MeOH} / \mathrm{CH}_{2} \mathrm{Cl}_{2}\right)$ brought the yellow colour off cleanly, however, a small amount of silica was solubilised with this solvent mixture and contaminated the sample. This was removed by filtering a solution of the product in $10 \% \mathrm{MeOH} / \mathrm{CH}_{2} \mathrm{Cl}_{2}$ through a fine frit, followed by chromatography on reversed phase silica. In this manner, 4.1 was isolated in $23 \%$ yield. The low yield is no doubt due to the difficulties experienced in purification of the product.

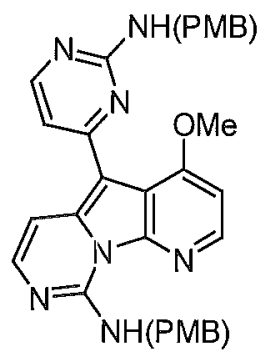

3.34
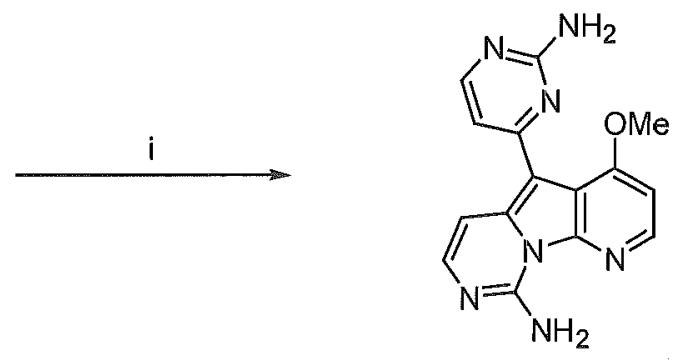

4.1

Scheme 4.1 Reagents and yields: (i) triflic acid/rt (23\%).

The synthesis of $O$-methylvariolin B (4.1) completes a three-membered group of compounds which vary only in their substituent at $\mathrm{C} 4$ (i.e. $\mathrm{Z}$ in Figure 4.1)-1.50 $(\mathrm{Z}=\mathrm{H}), \mathbf{1 . 1 7}(\mathrm{Z}=\mathrm{OH})$ and 4.1 $(\mathrm{Z}=\mathrm{OMe})$. Testing of these compounds should give an indication of the importance of this substituent for biological activity.

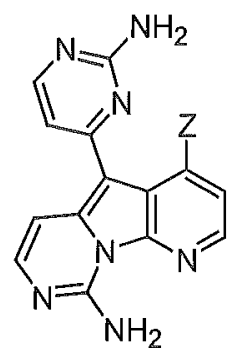

$1.50 \quad Z=H$ deoxyvariolin $B$

$1.17 \mathrm{Z}=\mathrm{OH}$ variolin $\mathrm{B}$

$4.1 Z=$ OMe O-methylvariolin $B$ 


\subsubsection{P388 Assay Results}

The results of the P388 assay of the three samples are shown in Table 4.1. Immediately apparent is the rather startling result that deoxyvariolin B (1.50) is equally active in the P388 assay as variolin $B$ itself! The implications of this unexpected result are discussed below. The other result concerns $O$-methylvariolin B (4.1), which displays moderate activity in the assay, with an $\mathrm{IC}_{50}$ of $3,660 \mathrm{ng} / \mathrm{mL}$.

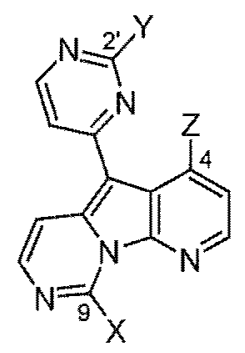

\begin{tabular}{c|cccc}
\hline & $\mathrm{X}$ & $\mathrm{Y}$ & $\mathrm{Z}$ & $\mathrm{IC}_{50}(\mathrm{ng} / \mathrm{mL})$ \\
\hline 1.50 & $\mathrm{NH}_{2}$ & $\mathrm{NH}_{2}$ & $\mathrm{H}$ & 157 \\
1.17 & $\mathrm{NH}_{2}$ & $\mathrm{NH}_{2}$ & $\mathrm{OH}$ & 189 \\
4.1 & $\mathrm{NH}_{2}$ & $\mathrm{NH}_{2}$ & $\mathrm{OMe}$ & 3,660 \\
\hline
\end{tabular}

Table 4.1 P388 assay results of variolin B (1.17), deoxyvariolin B (1.50) and $O$ methylvariolin B (4.1).

It is difficult to rationalise these results without making some assumptions regarding the role of the substitutent Z. Likewise, it is difficult to consider any possible roles of the substituent $Z$ without making some assumptions regarding the drug's mode of action.

It was suggested in the original isolation paper that variolin B may belong to the same class of DNA intercalating compounds as ellipticine and its analogues. ${ }^{32 a}$ Presumably, this statement was made on the basis of structural similarities between the molecules. Ellipticine (4.2) and 9. methoxyellipticine (4.3) are naturally occurring fused heteroaromatic systems ${ }^{117}$ with an overall planar structure. The discovery of their antitumour properties in $1967^{118}$ triggered widespread synthetic, biological and pharmacological studies of these compounds and numerous 
derivatives. ${ }^{119}$ As a consequence, their ability to intercalate into double-stranded DNA (i.e. slotting in between the stacked base pairs) has been well-established and this is believed to be responsible for their biological properties. ${ }^{120}$

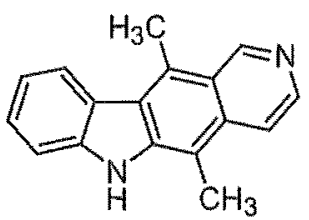

4.2

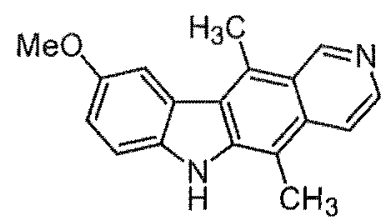

4.3

ellipticine

The ellipticine family is by no means unique in their intercalation into DNA. Numerous other natural and synthetic products have been shown to exhibit this behaviour. For example, synthetic dyes such as ethidium ion (4.4), proflavin (4.5) and acridine orange (4.6) are widely used as stains of DNA in biochemical laboratory processes. ${ }^{121}$ These agents bind by intercalation into double-stranded DNA. A general requirement for DNA intercalation is the presence of a planar substructure within the molecule, which is generally associated with an aromatic system.

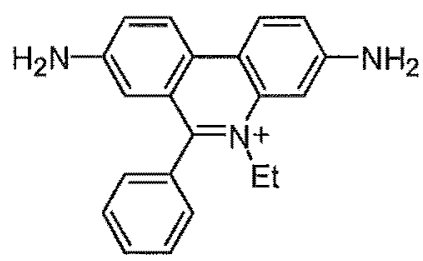

4.4

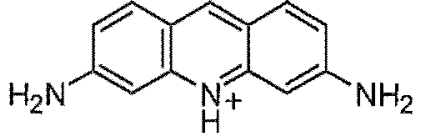

4.5<smiles>CN(C)c1ccc2cc3ccc(N(C)C)cc3[nH+]c2c1</smiles>

4.6

The comparison of variolin B (1.17) with ellipticine may, at first, seem somewhat unfounded. If variolin $\mathrm{B}$ was to exist in a planar conformation, significant clashing between $\mathrm{H} 6$ of the tricyclic core and H5' of the 2-aminopyrimidine ring would ensue. Steric arguments would suggest that the 2-aminopyrimidine ring plane should be significantly inclined to that of the tricyclic core to avoid this unfavourable interaction. However, as explained in Chapter One, an X-ray crystal structure of variolin $B$ showed that the two ring systems were closer to coplanarity than might be expected, being inclined to each other at an angle of $24^{\circ} .32 \mathrm{a}$ A hydrogen bond was observed 
between the hydroxyl proton and $\mathrm{N}^{\prime}$ of the 2-aminopyrimidine ring (see Figure 4.2 ); this may be important in allowing the molecule to adopt a near-planar structure.

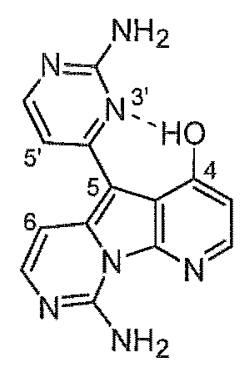

Figure 4.2 Internal hydrogen bonding seen in the X-ray crystal structure of variolin B (1.17).

If variolin $B$ acts as an intercalating agent, the ability to exist in a near-planar conformation may be important to its biological activity. The fact that $O$-methylvariolin B (4.1) displayed a $\sim 20$ fold decrease in activity compared with the natural product supports this notion. Hydroxyl protection would prevent any hydrogen bonding between the 2-aminopyrimidine ring and the tricyclic core, and would also force the 2-aminopyrimidine ring out of plane for steric reasons. It is interesting that variolin A (1.16) showed a comparable degree of potency in the P388 assay to 4.1, with an $\mathrm{IC}_{50}$ of $3,800 \mathrm{ng} / \mathrm{mL}^{32 \mathrm{~b}}$ The presence of the oxygen at $\mathrm{C}^{\prime}$ of the 2 aminopyrimidine ring would be expected to force this ring further out of the pyridopyrrolopyrimidine mean plane than for variolin B (1.17). Indeed, the crystal structure of variolin $\mathrm{A}(1.16)$ showed that the heterocyclic ring at $\mathrm{C} 5$ was inclined at an angle of $42^{\circ}$ to the tricyclic core-significantly more than was observed for variolin $\mathrm{B}^{32 \mathrm{~b}}$ It may be that the inability of both $O$-methylvariolin B (4.1) and variolin A (1.16) to attain coplanarity leads to their similar losses of activity as compared to variolin B (1.17).

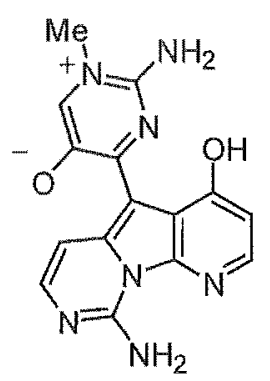


How then, does the finding that deoxyvariolin $B(1.50)$ is of equal cytotoxicity to variolin $B$ (1.17) fit in to this argument? It may be that deoxyvariolin B is quite capable of existing in a planar or near-planar conformation, without the need of a hydrogen bond to draw the 2aminopyrimidine ring into plane. In fact, the absence of the hydroxyl group at C4 may allow more freedom of movement of the 2-aminopyrimidine ring, such that a near-planar conformation is more easily attained.

It should be reiterated that the assumptions made in this discussion are speculative. To date, there is no direct evidence that the variolins are intercalating agents. To pursue this theory, DNA binding assays of variolin $B$ and its various analogues would be required, however such work was beyond the scope of this project. Neither can the results of the P388 assay be said to prove that a planar conformation is biologically important. Rather, this model provides a convenient means of rationalising the assay results. Nevertheless, both the structural similarity of the variolins to many known DNA intercalators, and these preliminary biological tests warrant further study in this area.

\subsubsection{Variation in Groups $X$ and $Y$}

\subsubsection{Synthesis}

To determine the importance of groups $X$ and $Y$, compounds 4.7 and 4.8 would be valuable. These compounds differ from variolin $\mathrm{B}$ only by the substitution of $\mathrm{X} / \mathrm{Y}=\mathrm{NH}_{2}$ with $\mathrm{X} / \mathrm{Y}=\mathrm{SMe}$. Unfortunately, the synthetic methodology does not lend itself to the production of 4.7, as selective substitution of the 2'-SMe group is not possible. However, relevant information regarding the importance of group $\mathrm{X}$ was gained from another analogue (vide infra).

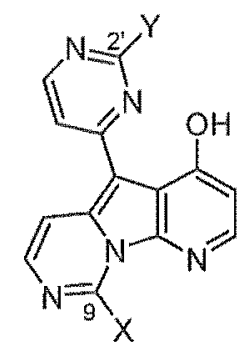

$4.7 X=S M e, Y=\mathrm{NH}_{2}$

$4.8 X=\mathrm{NH}_{2}, \mathrm{Y}=\mathrm{SMe}$ 
The synthesis of compound 4.8 began by selective substitution of the 9-SMe group of the variolin core structure 1.63. Thus, heating 1.63 in neat $p$-methoxybenzylamine cleanly gave the product of mono-substitution 4.9, in 86\% yield after flash chromatography on silica (Scheme 4.2). No evidence of di-substitution was observed. Deprotection of the methoxy group of 4.9 using sodium ethanethiolate at $50{ }^{\circ} \mathrm{C}$ gave $N$-protected pyridinol 4.10 in $95 \%$ yield. The ${ }^{1} \mathrm{H}$ NMR spectrum of this product indicated that trace amounts of 4.11 were present. This is the result of SMe substitution by sodium ethanethiolate. However, it was not possible to remove this product by silica flash chromatography. When pyridinol $\mathbf{4 . 1 0}$ was treated with triflic acid, the PMB group was cleaved to give the free amine 4.8. After neutralisation with aqueous ammonia solution, the ammonium triflate thus produced was able to be removed by washing a solution of the product in $5 \% \mathrm{MeOH} / \mathrm{CH}_{2} \mathrm{Cl}_{2}$ with aqueous $\mathrm{NaHCO}_{3}$ solution. Flash chromatography on silica allowed the isolation of $4.8\left(\sim 90 \%\right.$ pure by ${ }^{1}$ H NMR spectroscopy) in $80 \%$ yield.

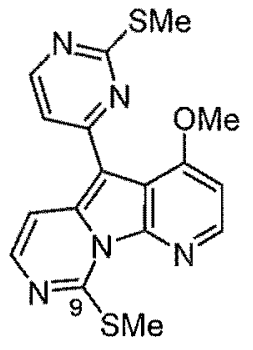

1.63

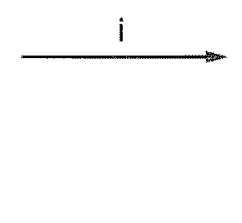

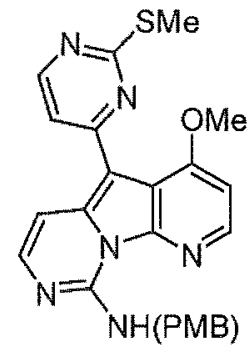

4.9<smiles>COc1ccnc2c1c(-c1ccnc(S(C)(=O)=O)n1)c1ccnc(N)n12</smiles>

4.12<smiles>[Y16]c1nccc(-c2c3c(O)ccnc3n3c(N)nccc23)n1</smiles>

4.10 $Y=S M e$

$4.11 Y=S E t$
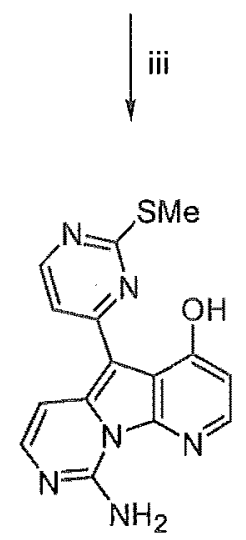

4.8

Scheme 4.2 Reagents and yields: (i) p-methoxybenzylamine (14 equiv)/85 ${ }^{\circ} \mathrm{C}$ (86\%); (ii) NaSEt (10 equiv)/DMF $/ 50{ }^{\circ} \mathrm{C}(4.1095 \%)$; (iii) triflic acid/rt (80\%); (iv) triflic acid/rt (100\%). 
A significant drop in solubility in halogenated solvents accompanies exposure of the free $\mathrm{NH}_{2}$ group, a phenomenon also noted with variolin $\mathrm{B}$, deoxyvariolin $\mathrm{B}$ and $O$-methylvariolin $\mathrm{B}$. Compound 4.8 was only sparingly soluble in either $\mathrm{CDCl}_{3}$ or DMSO- $\mathrm{d}_{6}$. NMR spectroscopy was carried out in a 1:1 mixture of these solvents.

As the $O$-methyl analogue 4.12 should also be readily obtainable from 4.9 , it was decided to include this compound in the present study. Removal of the PMB group of 4.9 was carried out in the usual manner-by standing in triflic acid for several hours - to give amine 4.12 in quantitative yield. Amine 4.12 was sufficiently soluble for NMR characterisation in $\mathrm{CDCl}_{3}$, including ${ }^{13} \mathrm{C}$ and $2 \mathrm{D}$ NMR spectroscopy.

As stated earlier, structure 4.7 would be useful in regard to the importance of group X, but cannot not be easily prepared by our methodology. However, an alternative analogue which differs from variolin $\mathrm{B}$ only in the nature of group $\mathrm{X}$ can be made. It was briefly mentioned in Chapter Two that $N$-deprotection using TFA appeared to remove only one of the PMB groups, although this particular product was not characterised. This selectivity in deprotection was investigated on the bis- $N$-protected pyridinol 3.35 .

Compound 3.35 was dissolved in TFA and left at room temperature, with the disappearance of starting material being followed by TLC. After 5 days, most of the starting material was consumed. Two new yellow products were evident, both of greater polarity than the starting material. It was assumed that these were the desired mono- $N$-protected product 4.13 and variolin $\mathrm{B}$ (1.17), the result of double- $N$-deprotection. Indeed, both of these products were present in the ${ }^{1} \mathrm{H}$ NMR spectrum of the crude material, in a $\sim 5: 1$ ratio in favour of 4.13 . Flash chromatography on silica allowed the isolation of 4.13 and variolin $\mathrm{B}(1.17)$ in 67 and 13\% yields respectively (Scheme 4.3). While variolin B did elute from this silica column, the purity was only moderate and therefore, reversed phase chromatography is generally recommended for this highly polar product. 


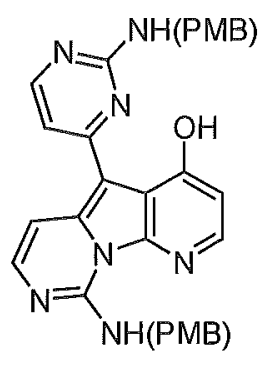

3.35

5

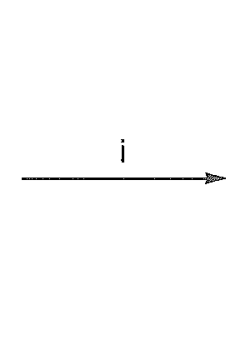

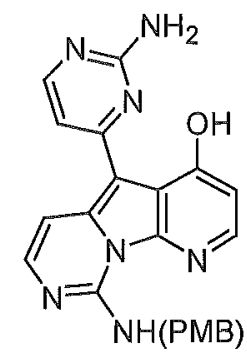

4.13<smiles></smiles>

1.17

Scheme 4.3 Reagents and yields: (i) TFA/rt (4.13 67\%, 1.17 13\%).

\subsubsection{P388 Assay Results}

The family of compounds thus generated was tested in the P388 assay, the results of which are shown in Table 4.2. It is immediately seen that none of these compounds display significant activity. One can conclude, therefore, that the variolin core skeleton is insufficient for biological activity-groups $\mathrm{X}$ and $\mathrm{Y}$ must play a role in the drug's cytotoxicity.

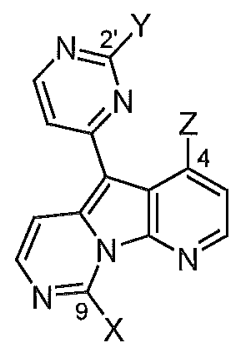

\begin{tabular}{c|cccc}
\hline & $\mathrm{X}$ & $\mathrm{Y}$ & $\mathrm{Z}$ & $\mathrm{IC}_{50}(\mathrm{ng} / \mathrm{mL})$ \\
\hline 1.17 & $\mathrm{NH}_{2}$ & $\mathrm{NH}_{2}$ & $\mathrm{OH}$ & 189 \\
4.8 & $\mathrm{NH}_{2}$ & $\mathrm{SMe}$ & $\mathrm{OH}$ & $>25,000$ \\
4.13 & $\mathrm{NH}(\mathrm{PMB})$ & $\mathrm{NH}_{2}$ & $\mathrm{OH}$ & $>25,000$ \\
4.9 & $\mathrm{NH}(\mathrm{PMB})$ & $\mathrm{SMe}$ & $\mathrm{OMe}$ & $>25,000$ \\
4.10 & $\mathrm{NH}(\mathrm{PMB})$ & $\mathrm{SMe}$ & $\mathrm{OH}$ & $>25,000$ \\
4.12 & $\mathrm{NH}_{2}$ & $\mathrm{SMe}$ & $\mathrm{OMe}$ & $>25,000$ \\
\hline
\end{tabular}

Table 4.2 P388 assay results of variolin B (1.17) and analogues. 
Even if only one of the groups $\mathrm{X}$ or $\mathrm{Y}$ is altered from those of variolin $\mathrm{B}(\mathbb{1} .17)$, this results in a $>100$-fold loss of activity. For example, compound 4.8 differs from variolin $B(1.17)$ only by the substitution of $\mathrm{Y}=\mathrm{NH}_{2}$ for $\mathrm{Y}=\mathrm{SMe}$. The inactivity of this compound indicates that the nature of this group is essential for biological activity. It cannot be said, however, that an amine is specifically required at this position until a variety of other substituents have been tested.

Compound 4.13 is more closely related to variolin $\mathrm{B}$ in that, both $\mathrm{X}$ and $\mathrm{Y}$ are amines, with $\mathrm{X}$ bearing a PMB group. However, even this analogue conveys no activity. This shows that an amine substituent at C9 is not, in itself, sufficient for cytotoxicity. Presumably, the PMB group is too bulky to allow interaction with the cellular target, whatever that target may be. Based on these results, it is not surprising that compounds $4.9,4.10$ and 4.12 are also devoid of biological activity.

While these assay results may seem disappointing, the purpose of producing these analogues was not to improve the potency of the drug, but rather to find out what features of variolin $\mathrm{B}$ are important to its biological function. In this regard, these studies have served their purpose very well. They have shown that the antitumour activity of variolin-type compounds is very sensitive to the nature of both $\mathrm{X}$ and $\mathrm{Y}$. Alteration of either group (in the above examples, at least) results in a $>100$-fold loss of activity compared to variolin B. This information is of relevance when it comes to designing further analogues of variolin B. 


\subsection{Semi-Rational Production of Variolin Analogues}

\subsubsection{Background}

Two alternative approaches can be taken to the synthesis of analogues of a lead compound. One approach is to make random incremental modifications to the structure-either by alteration of appendages or by modification of the gross architecture of the molecule - in the hope that some will display increased activity over the parent drug. A second approach centres around the systematic design of modified drugs, based on a knowledge of the structure of the drug's cellular target. In this rational design approach, one tries to maximise favourable interactions between the drug and its target by incorporating structural elements complementary to those found in the target. X-ray crystallography is a powerful technique in this regard as it can provide a "photograph" of the drug-target complex, plainly showing any interactions which occur at the interface of the complex.

While the cellular target of variolin $B$ is not known, the suggestion that it may act by intercalation into DNA is not unreasonable and deserves further attention. An examination of some current DNA intercalators that are promising anticancer agents reveals some important structural features. A group of drugs known as acridine-4-carboxamides have been widely studied for their antitumour properties. ${ }^{122}$ One of these, known as DACA or $\mathrm{N}$-[2(dimethylamino)ethyl]acridine-4-carboxamide (4.14), is currently in clinical trials as an antitumour agent. An X-ray crystal structure of a derivative of DACA, 9-amino-6-bromoDACA (4.15), complexed to a portion of duplex DNA demonstrates the specific interaction between the intercalator and DNA. ${ }^{122 a}$ In this complex, the planar acridine moiety slots between the $\mathrm{d}(\mathrm{CG})_{2}$ base pairs ( $\mathrm{d}$ refers to duplex DNA, $\mathrm{C}=$ cytosine, $\mathrm{G}=$ guanine) while the 2 (dimethylamino)ethyl chain can be seen to bind in the major groove, with a hydrogen bond between the $\mathrm{Me}_{2} \mathrm{NH}^{+}$of this side chain and N7 of one of the guanine bases. A similar complex has been characterised with 9-amino-DACA (4.16) and a duplex DNA hexanucleotide and shows similar interactions. ${ }^{122 \mathrm{c}}$ 


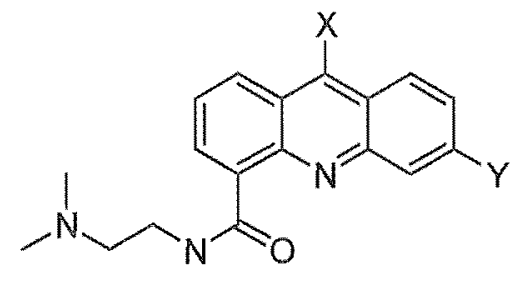

$4.14 X, Y=H$
$4.15 X=\mathrm{NH}_{2}, Y=\mathrm{Br}$
$4.16 \quad X=\mathrm{NH}_{2}, Y=\mathrm{H}$

Upon the recommendation of Professor Bill Denny of The University of Auckland, it was decided to incorporate a $\mathrm{Me}_{2} \mathrm{~N}\left(\mathrm{CH}_{2}\right)_{\mathrm{x}} \mathrm{NH}-($ where $\mathrm{x}=2$ or 3 ) side chain into either $\mathrm{C} 9$ or $\mathrm{C} 2$ ' of the variolin core. This "semi-rational" approach to analogue synthesis was based on the assumption that if variolin $\mathrm{B}$ does intercalate, then such a side chain may enhance binding affinity for DNA and, consequently, increase the activity of the drug. A useful set of analogues would incorporate the side chain at either $\mathrm{C} 9$ or $\mathrm{C}_{2}^{\prime}$, with an $\mathrm{NH}_{2}$ group at the other position, to give compounds $4.17-4.20$.

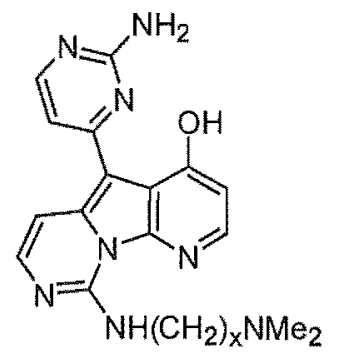

$4.17(x=2)$

$4.18(x=3)$

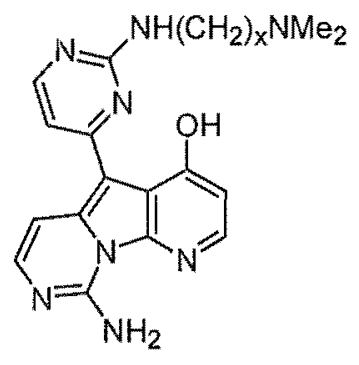

$4.19(x=2)$

$4.20(x=3)$

\subsubsection{Synthesis}

Incorporation of the side chain $\mathrm{Me}_{2} \mathrm{~N}\left(\mathrm{CH}_{2}\right)_{\mathrm{x}} \mathrm{NH}-($ where $\mathrm{x}=2$ or 3 ) into $\mathrm{C} 9$ of the variolin core structure 1.63 was readily achieved by heating the substrate at $100{ }^{\circ} \mathrm{C}$ with $\mathrm{Me}_{2} \mathrm{~N}\left(\mathrm{CH}_{2}\right)_{\mathrm{x}} \mathrm{NH}_{2}$, using toluene as solvent. This reaction would probably benefit from being conducted in a sealed tube, as addition of further portions of the amine were required throughout the course of the reaction-presumably due to loss of the reagent by evaporation. The mono-substituted products 4.21 and $\mathbf{4 . 2 2}$ were obtained in 84 and $89 \%$ yields respectively, after flash chromatography on either silica or alumina (Scheme 4.4). Product 4.22 (where $\mathrm{x}=3$ ) was considerably more polar on silica than 4.21 , however it could be purified readily by flash chromatography on alumina 
using much less polar solvent mixtures than were required on silica. Deprotection of the methoxy groups by heating with sodium ethanethiolate in DMF gave pyridinols 4.23 and 4.24 , along with small amounts of the inseparable SEt substituted products 4.25 and 4.26 . Both 4.23 and 4.24 required very polar solvent systems for flash chromatography on silica (20-30\% $\mathrm{MeOH} / \mathrm{CH}_{2} \mathrm{Cl}_{2}+0.5 \% \mathrm{NEt}_{3}$ ). Attempted purification of 4.24 (the more polar compound of the two) on alumina or reversed phase silica was unsuccessful and contributed to the lower yield of $4.24(58 \%)$ compared with that of $4.23(91 \%)$.

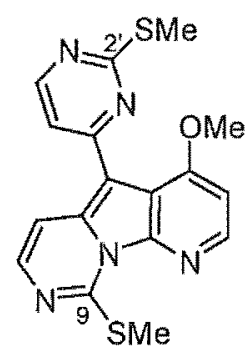

1.63
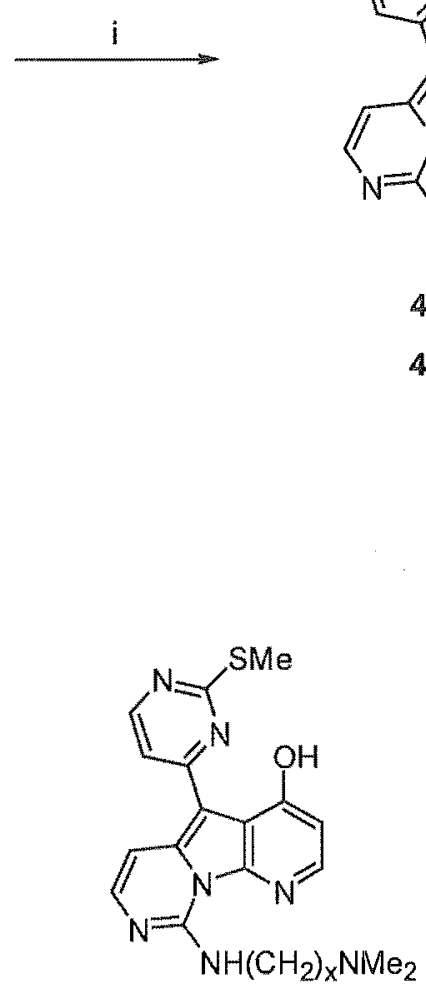

$4.23(x=2)$

$4.24(x=3)$<smiles>CCCCCNc1nccc2c(-c3ccnc(SC)n3)c3c(OC)ccnc3n12</smiles>

$4.21(x=2)$

$4.22(x=3)$

$$
\mid
$$

Scheme 4.4 Reagents and yields: (i) $\mathrm{Me}_{2} \mathrm{~N}_{4}\left(\mathrm{CH}_{2}\right)_{\mathrm{x}} \mathrm{NH}_{2}$ (2-6 equiv)/toluene/100 ${ }^{\circ} \mathrm{C}(4.2184 \%, 4.22$ $89 \%$ ); (ii) NaSEt (7 equiv)/DMF $/ 60^{\circ} \mathrm{C}(4.2391 \%, 4.2458 \%$ ).

Introduction of an amine at $\mathrm{C}_{2}$ ' requires prior oxidation of the remaining thiomethyl group. Unfortunately, attempted $S$-oxidation of $\mathbf{4 . 2 2}$ using $m$-CPBA did not cleanly produce the 
corresponding sulfoxide or sulfone. The products of these reactions were not isolated but are thought to be products of tertiary $N$-oxidation. Even when the reaction was carried out at $-60{ }^{\circ} \mathrm{C}$ with only one equivalent of $m$-CPBA, it appeared that $N$-oxidation had taken place preferentially to $S$-oxidation. This conclusion was based on the ${ }^{1} \mathrm{H}$ NMR chemical shifts of the $\mathrm{SCH}_{3}$ signal, the $\mathrm{N}\left(\mathrm{CH}_{3}\right)_{2}$ signal and the $-\mathrm{CH}_{2} \mathrm{NMe}_{2}$ signal. First, the $\mathrm{SCH}_{3}$ signal was essentially unchanged from the starting material, at $\delta$ 2.63. However, the $\mathrm{N}\left(\mathrm{CH}_{3}\right)_{2}$ signal (easily identifiable as it integrates for six protons) appeared at $\delta 3.45$-greater than $1 \mathrm{ppm}$ downfield than was observed for the starting material. Similarly, the $-\mathrm{CH}_{2} \mathrm{NMe}_{2}$ signal, which was at $\delta 2.47$ in the starting material, appeared at $\delta 3.78$ in the new product. These values indicated that it was the nitrogen which had been oxidised rather than the sulfur.

This result appeared to be a minor drawback in the general usefulness of this system for analogue production, as selective introduction of a nucleophile followed by $S$-oxidation was a central element in our plan. However, an alternative option for the selective incorporation of different groups at $\mathrm{C} 9$ and $\mathrm{C} 2{ }^{\prime}$ was available. It may be recalled from Chapter Three that attempted chromatography of the bis-sulfoxide 3.32 using $\mathrm{MeOH} / \mathrm{CH}_{2} \mathrm{Cl}_{2}$ mixtures as the eluant led to the formation of a new product, thought to be structure 3.33 .

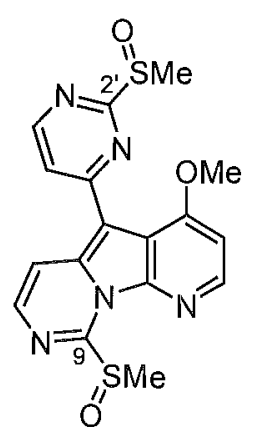

3.32

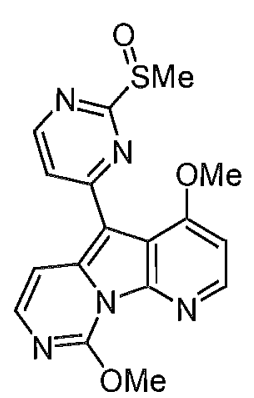

3.33

This product is thought to arise from nucleophilic substitution of the 9-SOMe group at room temperature. Based on this result, the selective introduction of an amine nucleophile at C9 of 3.32, without affecting the sulfoxide group at $\mathrm{C}^{\prime}$, may be feasible. Subsequent incorporation of a second substituent could be achieved at elevated temperature, as the sulfur is already at the required oxidation state for substitution to occur. 
To investigate this possibility, the bis-sulfoxide was produced by reaction with 2 equivalents of $m$-CPBA at $-60^{\circ} \mathrm{C}$, rather than the usual $-40^{\circ} \mathrm{C}$ (Scheme 4.5 ). The lower temperature resulted in very clean production of 3.32; no SMe peaks could be detected in the ${ }^{1} \mathrm{H}$ NMR spectrum, although some of the putative sulfoxide/sulfone 4.27 was produced. For the introduction of the amine, it was decided to conduct the reaction in $\mathrm{CDCl}_{3}$ so that the reaction could be easily followed by NMR spectroscopy. Thus, 1 equivalent of 3-(dimethylamino)propylamine was added to a solution of 3.32 and the corresponding sulfone 4.27 in $\mathrm{CDCl}_{3}$, and the mixture was examined by ${ }^{1} \mathrm{H}$ NMR spectroscopy after 10 minutes. Pleasingly, most of the starting material had been consumed to give what appeared to the product of mono-substitution. No substitution of the $2^{\prime}-\mathrm{S}(\mathrm{O})_{\mathrm{x}} \mathrm{Me}$ groups could be detected.

To ensure complete reaction, the solution was left for a total of $17 \mathrm{~h}$ at room temperature. The reaction mixture was purified by flash chromatography on silica (using $30 \% \mathrm{MeOH} / \mathrm{CH}_{2} \mathrm{Cl}_{2}+$ $1 \% \mathrm{NEt}_{3}$ as eluant), followed by reversed phase chromatography (using $50 \% \mathrm{MeOH} /$ water + $0.1 \%$ TFA), to give a mixture of amines 4.28 and 4.29 (Scheme 4.5). ${ }^{1} \mathrm{H}$ NMR spectroscopy indicated the ratio of sulfoxide 4.28 and sulfone 4.29 to be $\sim 8: 1$. These were not separated and the mixture was enriched enough in the sulfoxide to allow spectroscopic characterisation of this product. The extreme polarity of these compounds should be mentioned. The mixture of products was quite readily soluble in water, and yet was still easily characterised in $\mathrm{CDCl}_{3}$. The (dimethylamino)propyl chain appears to be responsible for this solubilising effect.

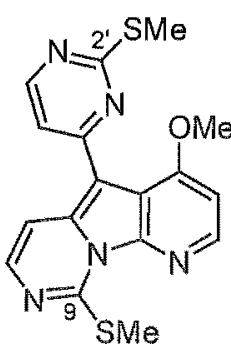

1.63

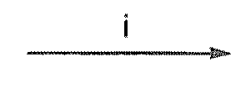

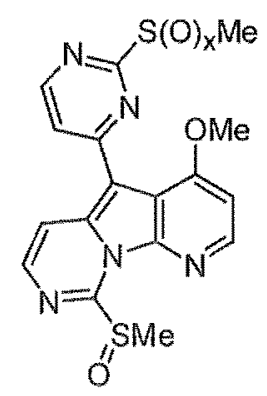

$3.32(x=1)$

$4.27(x=2)$

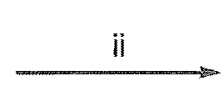<smiles>CCCCCCCCCCCCCCCCNc1nccc2c(-c3ccnc([Si](OC)OC)n3)c3c(OC)ccnc3n12</smiles>

$4.28(x=1)$

$4.29(x=2)$

Scheme 4.5 Reagents and yields: (i) $m$-CPBA (2 equiv) $/ \mathrm{CHCl}_{3} /-60{ }^{\circ} \mathrm{C}$ to $\mathrm{rt}$; (ii) 3 -(dimethylamino)propylamine (1 equiv)/ $\mathrm{CDCl}_{3} / \mathrm{rt}(8: 1$ mixture of 4.28 and $4.29,44 \%$ from 1.63 ); (iii) $\sim 25 \%$ aq $\mathrm{NH}_{3} / 95^{\circ} \mathrm{C}$, sealed tube. 
Substitution of the sulfoxide/sulfone groups with an amine and subsequent methoxy deprotection would give compound $\mathbf{4 . 1 8}$, an analogue directly comparable to variolin B (1.17), in which the C9 substituent $\mathrm{X}=\mathrm{NH}_{2}$ has been swapped with $\mathrm{X}=\mathrm{NH}\left(\mathrm{CH}_{2}\right)_{3} \mathrm{NMe}_{2}$. Due to a limited amount of material available in this late stage of the project, it was decided to directly introduce the amino group using aqueous ammonia rather than our $p$-methoxybenzylamine/deprotection protocol. Álvarez et al. had introduced the $2^{\prime}-\mathrm{NH}_{2}$ group of deoxyvariolin $\mathrm{B}$ in this manner, in good yield $(90 \%)$. $^{38}$

However, when the mixture of sulfoxide 4.28 and sulfone 4.29 was heated in aqueous ammonia solution, mixtures of products were obtained. Characterisation could not be achieved due to difficulties in purifying the mixture, and a lack of material prevented further investigation of this reaction. In the future, it may be prudent to use the protected amine with subsequent removal of the PMB group, as this has always resulted in clean substitution.

While the desired analogues $4.17-4.20$ have not yet been produced, the intermediates $4.21-4.24$ were tested for antitumour activity in the P388 assay.

\subsubsection{P388 Assay Results}

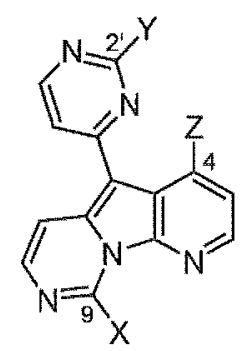

\begin{tabular}{c|cccc}
\hline & $\mathrm{X}$ & $\mathrm{Y}$ & $\mathrm{Z}$ & $\mathrm{IC}$ \\
\hline 4.8 & $\mathrm{NH}_{2}$ & $\mathrm{SMe}$ & $\mathrm{OH} / \mathrm{mL})$ \\
4.21 & $\mathrm{NH}\left(\mathrm{CH}_{2}\right)_{2} \mathrm{NMe}_{2}$ & $\mathrm{SMe}$ & $\mathrm{OMe}$ & $>25,000$ \\
4.23 & $\mathrm{NH}\left(\mathrm{CH}_{2}\right)_{2} \mathrm{NMe}_{2}$ & $\mathrm{SMe}$ & $\mathrm{OH}$ & 10,630 \\
4.22 & $\mathrm{NH}\left(\mathrm{CH}_{2}\right)_{3} \mathrm{NMe}_{2}$ & $\mathrm{SMe}$ & $\mathrm{OMe}$ & 12,930 \\
4.24 & $\mathrm{NH}\left(\mathrm{CH}_{2}\right)_{3} \mathrm{NMe}_{2}$ & $\mathrm{SMe}$ & $\mathrm{OH}$ & 3460 \\
\hline
\end{tabular}

Table 4.3 P388 assay results of several variolin analogues. 
Table 4.3 shows the results of the four (dimethylamino)alkyl variolin analogues. Included in the Table is the result of compound $\mathbf{4 . 8}$ from the earlier assay, so that a specific analysis can be made regarding the effect of substituting an amino group with a (dimethylamino)alkyl chain. Pleasingly, all of the analogues $4.21-4.24$ showed increased activity over the free amine 4.8 . This gives an indication that inclusion of the (dimethylamino)alkyl chain may be a useful method for increasing the activity of the variolins.

When comparing the two different chains, it can be seen that the 3-carbon chain $(4.22$ and 4.24$)$ gives greater activity than the 2-carbon chain (4.21 and 4.23). It is also apparent from these results that deprotection of the methoxy group results in a slight drop in activity. This was unexpected based on the assay results of variolin B (1.17) and $O$-methylvariolin B (4.1) shown in Table 4.1, where the natural product showed 20 -fold greater activity than the $O$-methyl analogue 4.1 .

It is difficult to rationalise these results without any knowledge of how these compounds are interacting with the biological system. That will be the focus of a separate study. The present results show that antitumour activity can be enhanced by appropriate substitution of the $9-\mathrm{NH}_{2}$ group. Based on these results and the results discussed earlier in the Chapter, it is reasonable to suggest that replacement of the thiomethyl group of compounds $4.21-4.24$ with $\mathrm{NH}_{2}$ would further increase the activity, perhaps even beyond that of variolin $B$. 


\subsection{Summary}

Clearly, further work is required in this area. This will be detailed in the following Chapter. However, it is hoped that this brief examination has demonstrated the versatile nature of this synthesis for analogue production. Displacement of the 9-SMe group proceeds cleanly and selectively with all nucleophiles that have been investigated so far. Subsequent oxidation of the 2'-SMe group should be straightforward provided that the $\mathrm{C} 9$-substituent does not contain any oxidisable groups. However, if this presents a problem, it has been shown that oxidation of both thiomethyl groups to the sulfoxides, followed by selective substitution of the 9-SOMe group at room temperature, is a viable process. Subsequent substitution of the 2'-SOMe at elevated temperature should not present any problems. Alternatively, if the same group is required at both $\mathrm{C} 9$ and $\mathrm{C} 2$ ', these can be introduced in the same step.

Regarding the biological assays that have been carried out, it can be said that the results gained thus far are certainly of interest and provoke some thought regarding the biological role of the variolin compounds. Apart from the pleasing result that synthetic variolin B showed at least equal potency to the natural material, the most significant result concerned deoxyvariolin B (1.50). The fact that this analogue is also equipotent was unexpected, yet not unreasonable. In light of the fact that $O$-methylvariolin B (4.1) was 20 -fold less active, this may be regarded as tenuous evidence that a general overall planar structure is required for biological activity.

It has been shown that the degree of cytotoxicity is quite dependent on the nature of groups $\mathrm{X}, \mathrm{Y}$ and $\mathrm{Z}$ (at $\mathrm{C} 9, \mathrm{C} 2{ }^{\prime}$ and $\mathrm{C} 4$, respectively). Preliminary results show that incorporation of a (dimethylamino)alkyl chain at $\mathrm{C} 9$ can lead to a significant increase in cytotoxicity. These results certainly warrant a more thorough investigation into the effects of different substituents at these three points of variation. 


\title{
Chapter Five
}

General Summary

\author{
and
}

\section{Future Studies}




\subsection{General Summary}

This dissertation has covered the development of methodology that has culminated in the first total synthesis of variolin B (1.17). At the commencement of this work, no synthetic efforts directed towards the variolins had been reported in the literature. However, throughout the course of the work described in this thesis, several publications emerged regarding the syntheses of either fragments of the pyrido $\left[3^{\prime}, 2^{\prime}: 4,5\right]$ pyrrolo[1,2-c]pyrimidine ring system, ${ }^{35}$ or this system itself with subsituents attached at various positions. ${ }^{36,37,38}$ All of these syntheses consist of fundamentally the same retrosynthetic strategy-disconnection of the C9-N10 bond to give a 2substituted 7-azaindole (Figure 5.1a). The strategy used in this thesis employs an alternative disconnection, breaking the N10-C10a bond of the variolin core (Figure 5.1b).

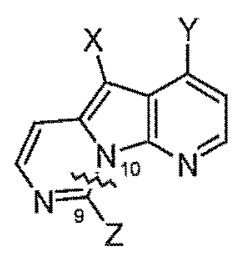

a

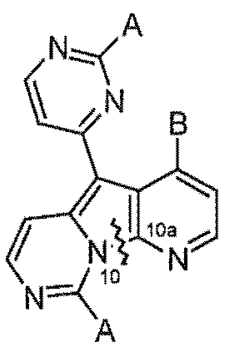

b

Figure 5.1 Retrosynthetic strategies for construction of the variolin core.

To investigate this strategy, the triarylmethanol 2.3 was required. Chapter Two describes the synthesis of triarylmethanol 2.3 by lithiation of iodopyrimidine 2.12 and subsequent lowtemperature addition to the acid chloride 2.4 (Scheme 5.1). After considerable experimentation, procedures were found to minimise the formation of several unwanted side-products, enabling the triarylmethanol to be produced in $56 \%$ yield for the double addition. Following this, a number of methods were attempted for forming the variolin core structure 2.1. Most of these were unsuccessful, being either too harsh for the present system or too unreactive. However, it was found that heating triarylmethanol 2.3 with a combination of triethylsilane and trifluoroacetic acid led to the formation of the variolin core structure 2.1 in low yield. Optimisation of this reaction allowed the core structure to be isolated in $34 \%$ yield. While this yield is modest, it should be noted that 2.1 is produced in only three steps from 2.13. A number 
of side-products isolated from this reaction give insights into the mechanism of cyclisation, which appears to proceed by a quite different mechanism to that originally proposed.

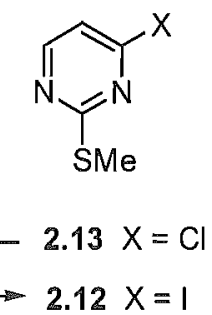

$2.12 x=1$

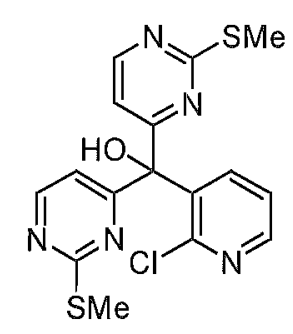

2.3

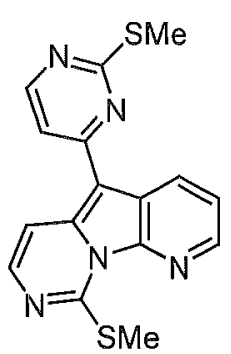

2.1

Scheme 5.1 Synthesis of triarylmethanol 2.3 and cyclisation to the variolin core structure 2.1.

The elaboration of core structure 2.1 to deoxyvariolin B (11.50) is also described in this Chapter. Oxidation of both thiomethyl groups using $m$-CPBA gave the bis-sulfoxide 2.69 , which was readily substituted with 2 equivalents of $p$-methoxybenzylamine to give the bis-protected amine 2.70 (Scheme 5.2). Removal of the PMB groups was accomplished using triflic acid at room temperature to give deoxyvariolin B (1.50) in six steps from commercially available 2-chloro-4(methylsulfanyl)pyrimidine (2.13).

2.1

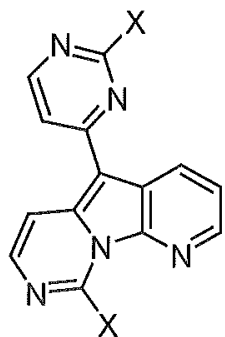

$\left[\begin{array}{l}2.69 X=\text { SOMe } \\ 2.70 X=N H(P M B)\end{array}\right.$<smiles>Nc1nccc(-c2c3cccnc3n3c(N)nccc23)n1</smiles>

1.50

deoxyvariolin $\mathrm{B}$

Scheme 5.2 Elaboration of variolin core structure 2.1 to deoxyvariolin B (1.50). 
Application of the methodology described above to a synthesis of variolin B required access to acid chloride 1.67 (Scheme 5.3). As detailed in Chapter Three, this was produced by a modification of Kasum and Prager's synthesis of the bromo-analogue. ${ }^{96}$ However, the double addition of lithio-species $\mathbf{1 . 6 6}$ to this acid chloride was unreliable, with the desired triarylmethanol 1.65 never being isolated in greater than $15 \%$ yield. Despite investigating other pyrimidinyl-metal species (magnesium, cerium) and different conditions, no improvement could be made to the yield of 1.65 .<smiles>CSc1nccc(I)n1</smiles>

2.12<smiles>CSc1nccc([AlH2])n1</smiles>

1.66<smiles>COc1ccnc(Cl)c1C(=O)Cl</smiles><smiles></smiles>

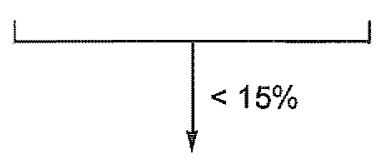<smiles>COc1ccnc(Cl)c1C(O)(c1ccnc(SC)n1)c1ccnc(S(C)(=O)=O)n1</smiles>

1.65

Scheme 5.3 Initial synthesis of triarylmethanol 1.65.

Accordingly, a new synthesis of the triarylmethanol was developed. The known ${ }^{103-105} 2$-chloro4-methoxypyridine (3.8) was produced in three steps from commercially available 4nitropyridine- $N$-oxide (Scheme 5.4). It was found that clean metalation at C3 of 3.8 could be achieved using $n-\mathrm{BuLi}$ at low temperature (below $-78^{\circ} \mathrm{C}$ ). The resulting lithiated pyridine was to be reacted with symmetrical ketone 3.15 (produced by reaction of 2 equivalents of the lithiospecies 1.66 with diethyl carbonate). In the event, the reaction of these two species at $-78^{\circ} \mathrm{C}$ resulted in the formation of the desired triarylmethanol 1.65 in a reproducible yield of $76 \%$. 
<smiles>CSc1nccc(I)n1</smiles>

2.12<smiles>CSc1nccc(C(=O)c2ccnc(S(C)(=O)=O)n2)n1</smiles>

3.15<smiles>COc1ccnc(Cl)c1</smiles>

3.8

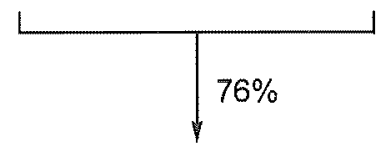<smiles>Cc1ccnc(Cl)c1C(O)(c1ccnc(S(C)(=O)=O)n1)c1ccnc(S(C)(=O)=O)n1</smiles>

Scheme 5.4 Improved synthesis of triarylmethanol 1.65 .

Unfortunately, subjection of triarylmethanol $\mathbf{1 . 6 5}$ to the optimum conditions for the formation of deoxy-core structure 2.1 resulted in the formation of 1.63 in only $9 \%$ isolated yield. However, further optimisation of this system improved the yield of $\mathbf{1 . 6 3}$ to $47 \%$ (Scheme 5.5). The major side-product in this reaction, uncyclised ether 3.25 , was isolated in $28 \%$ yield. It was reasoned that this product could be eliminated by carrying out the cyclisation reaction on an alternative substrate, triarylmethylacetate 3.26. The acetate was produced in the same fashion as the triarylmethanol 1.65, except that the reaction was quenched with acetyl chloride. To our delight, when 3.26 was treated to the same cyclisation conditions that were used for the triarylmethanol 1.65, the desired variolin core 1.63 was obtained in $75 \%$ yield, with no trace of the uncyclised product 3.25 being detected. 
<smiles>COc1ccnc(Cl)c1C(O)(c1ccnc(S(C)(=O)=O)n1)c1ccnc(S(C)(=O)=O)n1</smiles>

1.65

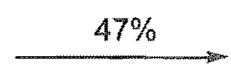

3.15

3.8<smiles>COc1ccnc2c1c(-c1ccnc(SC)n1)c1ccnc(SC)n12</smiles>

1.63<smiles>COc1ccnc(Cl)c1C(Oc1ccnc(SC)n1)c1ccnc(SC)n1</smiles>

3.25 $75 \%$<smiles>COc1nccc(C(O)(c2ccnc(SC)n2)c2c(OC)ccnc2Cl)n1</smiles>

3.26

Scheme 5.5 Synthesis of the variolin core 1.63 from triarylmethanol $\mathbf{1 . 6 5}$ and improved synthesis from the acetate $\mathbf{3 . 2 6}$.

Core structure 1.63 was elaborated to the target, variolin B (1.17), following precedent established in the deoxy-series. However, one extra step is required for deprotection of the methoxy group. While boron tribromide was unsuccessful in this regard, sodium ethanethiolate proved very effective. However, due to nucleophilic substitution of the SMe groups when this step was carried out on $\mathbf{1 . 6 3}$, it was deemed more appropriate to conduct this step once the protected amines had been introduced. Thus, $S$-oxidation and nucleophilic substitution led to the bis-protected amine 3.34 , the methoxy group of which was readily cleaved using sodium ethanethiolate (Scheme 5.6). To complete the synthesis, the PMB groups were removed with triflic acid to produce the target compound, variolin B (1.17). The overall yield for the eight step sequence was $13 \%$, calculated from 2-chloro-4-(methylsulfanyl)pyrimidine $(2.13)$.

\footnotetext{
"Two months after the publication of this work, Molina, Fresneda and coworkers published their total synthesis of variolin $B$, which proceeded in 16 steps and an overall yield of $1,2 \%{ }^{36 \mathrm{~b}}$
} 


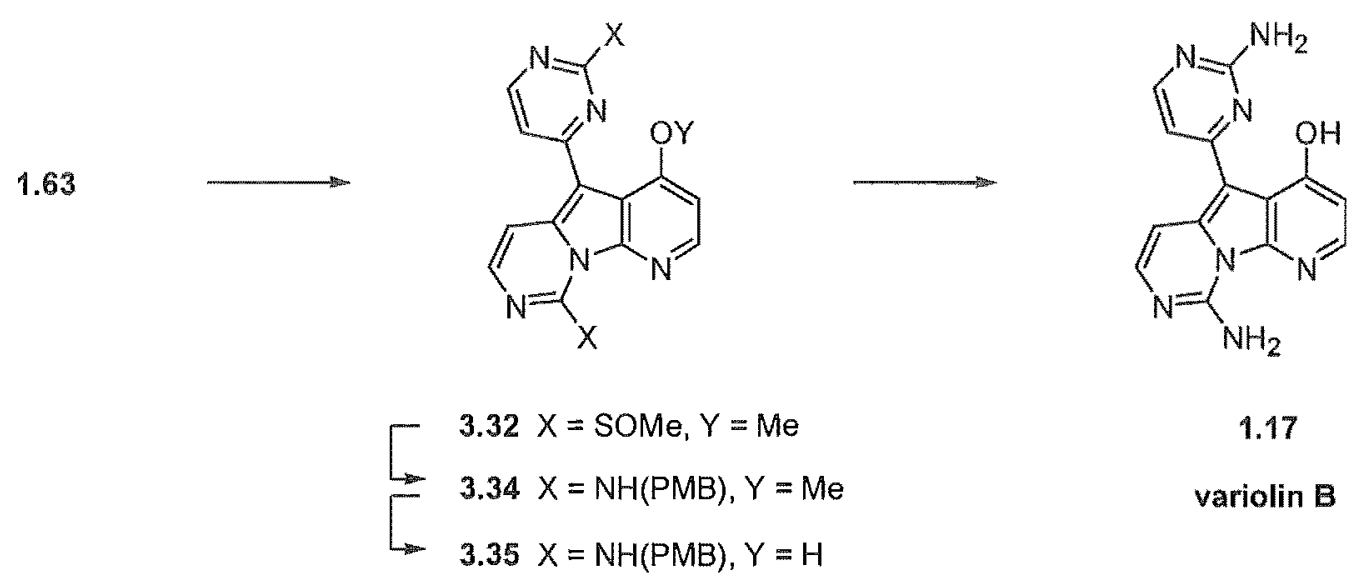

Scheme 5.6 Elaboration of variolin core structure 1.63 to variolin B (1.17).

Significantly, now that a supply of synthetic variolin B has been established, further biological evaluation is now able to be carried out. As has been mentioned, preliminary results against a range of human tumour cell lines have seen variolin B enter into Safety Trials. This represents an important step forward in the development of variolin B into a therapeutic agent.

Chapter Four highlights the utility of this synthesis for the production of analogues. Some preliminary undertakings in this area and the biological testing of these analogues were carried out, revealing that several analogues displayed modest to good activity in the P388 assay. It is hoped that further work in this area will bring to light other compounds of biological interest, and that ultimately, these studies will play a part in the effective treatment of cancer in its various forms. 


\subsection{Future Studies}

The synthetic strategy utilised in the syntheses of both deoxyvariolin $B(1.50)$ and the natural product, variolin B (1.17) must be fully exploited in the production of a wide range of analogues. In this regard, solid phase and/or combinatorial synthesis would be particularly suited to the variolins due to the high yielding and clean substitution of the thiomethyl groups, and facile deprotection of the methoxy group. The establishment of a library of compounds, varying in groups X, Y and Z (see Figure 5.2) would provide the basis for enhancement of the drug's biological activity and the fine-tuning of important pharmacological properties (stability, solubility, toxicity etc.). In particular, the family of (dimethylamino)alkyl analogues discussed in Chapter Four requires further investigations due to the promising early results. The synthesis of analogues $4.17-4.20$ must be a specific goal in this area, with a particular emphasis on those compounds with a 3 -carbon chain.

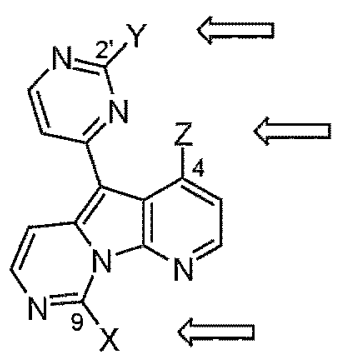

Figure 5.2 Points of variation available in the variolins.

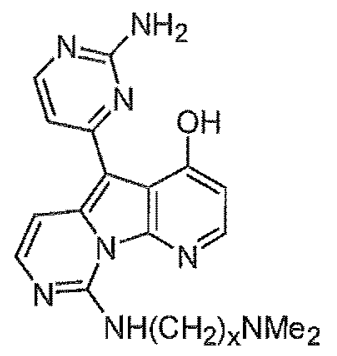

$4.17(x=2)$

$418(x=3)$

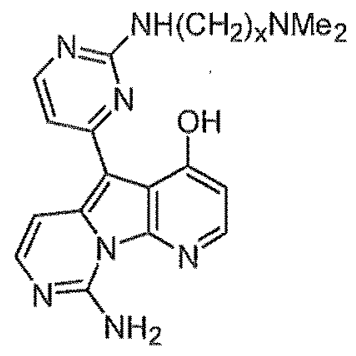

$4.19(x=2)$

$4.20(x=3)$ 
Analogues which differ from variolin B in their gross architecture may also be accessible and of biological value. For example, the oxygen-containing products 2.42 and 2.44 , which were obtained in the cyclisation reaction to form the deoxy-core structure $\mathbf{2 . 1}$, would be expected to possess a slightly different shape from that of the natural product, which may result in increased or modified biological activity. Replacement of the thiomethyl groups with $\mathrm{NH}_{2}$ would be necessary to make a direct comparison of the shapes and bioactivities of these compounds with deoxyvariolin B and variolin B (Figure 5.3).<smiles>CSc1nccc(Oc2c3cccnc3n3c(SC)nccc23)n1</smiles>

2.42<smiles>CSC1=NC=CC2(c3ccnc(SC)n3)C(=O)c3cccnc3N12</smiles>

2.44<smiles>Nc1nccc(Oc2c3cccnc3n3c(N)nccc23)n1</smiles><smiles>NC1=NC=CC2(c3ccnc(N)n3)C(=O)c3cccnc3N12</smiles>

Figure 5.3 Possible analogues of variolin B, differing in their overall shape.

As has been mentioned, the successful total synthesis of variolin B has allowed its further biological investigation, which is necessary for its potential development into a medicinal agent. To complement these efforts, studies into the mode of action would allow the full potential of this compound to be realised. It has been suggested that variolin B may form a complex with DNA by intercalation. Accordingly, DNA binding studies ${ }^{123}$ may help to determine what, if any, interaction variolin B has with DNA. If a positive connection is established, NMR studies and 
X-ray crystallography may be useful in determining the sequence-specificity of the binding interaction and may identify specific points of contact between the drug and the DNA sequence. This would allow a more rational approach to the design of analogues with higher binding affinities and/or increased potency. It would be interesting to compare the outcome of this approach to drug-design to the results of producing a library of essentially random variolin analogues.

However, an open mind must always be maintained as to the biological role of variolin B. Only once the cellular target is firmly established can any concrete hypothesis be given regarding the mode of action. Studies in this area will not only reveal how variolin B interacts with cellular processes, but may also open up new avenues to block the processes that cause the proliferation of cancerous cells. 


\section{Chapter Six}

\section{Experimental}




\subsection{General Experimental}

\section{Physical and Spectroscopic Techmiques}

Melting points were obtained on either an Electrothermal heating block or a Reichert microscope hot-stage and are uncorrected.

${ }^{1}$ H NMR spectra were obtained on either a Varian Unity 300 or Varian Inova 500 spectrometer, operating at 300 and $500 \mathrm{MHz}$ respectively. ${ }^{13} \mathrm{C}$ NMR were obtained on either a Varian Unity 300 or a Varian XL 300 instruments at $75 \mathrm{MHz}$, or a Varian Inova 500 spectrometer operating at $126 \mathrm{MHz}$, typically with a delay $\left(\mathrm{d}_{1}\right)$ of $2-3 \mathrm{~s}$. In some cases, the Inova 500 was fitted with a Broadband Probe for ${ }^{13} \mathrm{C}$ NMR spectra of very small or insoluble samples, as required; otherwise an Inverse Detection Probe was used. Chemical shifts are reported in parts per million (ppm) on the $\delta$ scale, and were referenced to residual protonated solvent peaks: deuteriochloroform spectra were referenced to $\mathrm{CHCl}_{3}$ at $\delta_{\mathrm{H}} 7.26$ and $\mathrm{CDCl}_{3}$ at $\delta_{\mathrm{C}} 77.0 ; \mathrm{DMSO}-\mathrm{d}_{6}$ spectra were referenced to $\left(\mathrm{CHD}_{2}\right)\left(\mathrm{CD}_{3}\right) \mathrm{SO}$ at $\delta_{\mathrm{H}} 2.50$ and $\left(C_{3}\right)_{2} \mathrm{SO}$ at $\delta_{\mathrm{C}} 39.6 ;$ methanol-d $\mathrm{d}_{4}$ spectra were referenced to $(\mathrm{CHD}) \mathrm{OD}$ at $\delta_{\mathrm{H}} 3.30$.

2D NMR experiments were carried out on either a Varian Unity 300 or Varian Inova 500 spectrometer, operating at 300 and $500 \mathrm{MHz}$ respectively. Both instruments were fitted with an Inverse Detection Probe and Pulsed Field Gradient driver. Assignments of $\mathrm{H}$ and $\mathrm{C}$ signals are given on the basis of COSY, HSQC, and HMBC or CIGAR experiments. In some cases, difference NOE or ID NOESY experiments were employed if uncertainty regarding structural assignments remained. HMBC and CIGAR experiments were usually obtained with an arrayed value of ${ }^{n} J_{\mathrm{CH}}=4,9 \mathrm{~Hz}$.

IR spectra were obtained on a Shimadzu FTIR-8201 PC spectrophotometer. Spectra of solids were obtained from $\mathrm{KBr}$ pellets or by the diffuse reflectance method, or in a solution of $\mathrm{CDCl}_{3}$ using $\mathrm{CaF}_{2}$ solution cells. Oils were run neat on $\mathrm{KBr}$ plates. Values are reported in wavenumbers $\left(\mathrm{cm}^{-1}\right)$. 
Electron impact mass spectrometry was carried out on a Kratos MS80RFA at $70 \mathrm{eV}$ and $4 \mathrm{kV}$ accelerating potential. Electrospray mass spectrometry was carried out on a Micromass LCT at $3.2 \mathrm{kV}$ and $150^{\circ} \mathrm{C}$ (probe temperature), with nitrogen nebulisation at $150 \mathrm{~L} / \mathrm{h}$ and desolvation at $500 \mathrm{~L} / \mathrm{h}$.

\section{Chromatography}

Preparative HPLC was performed on a Shimadzu LC-4A instrument equipped with a Hewlett Packard 3390A integrator, using a reversed phase Dynamax ${ }^{\circledR}$ column $(60 \AA ; 250 \times 10 \mathrm{~mm})$ with a flow rate of $10-13 \mathrm{~mL} / \mathrm{min}$.

MPLC was perfomed on a Merck Lobar ${ }^{(B)}$ size B (310-25) LiChroprep ${ }^{(R)}$ (RP-18) column (40-63 $\mu \mathrm{m})$, connected to a Mill-Royal D pump with a flow rate of $5.3 \mathrm{~mL} / \mathrm{min}$. The system was equipped with a LKB Broma 2238 UVICORD SII detector and a LKB Broma 2210 recorder with detection at $254 \mathrm{~nm}$.

Analytical thin layer chromatography (TLC) was conducted on aluminum-backed Merck Kieselgel $\mathrm{KG}_{60 \mathrm{~F}_{254}}$ silica plates or aluminum-backed alumina type $\mathrm{H}$ plates. Plates were visualised by both short- and long-wave UV light. For compounds possessing the pyrido[ $\left.3^{\prime}, 2^{\prime}: 4,5\right]$ pyrrolo[1,2-c]pyrimidine core, long-wave UV light was particularly useful.

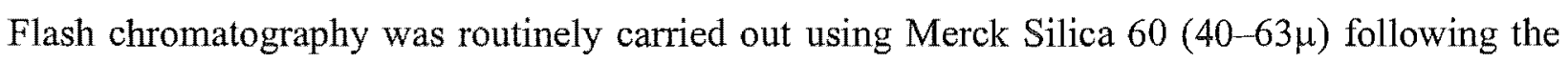
procedure of Still and coworkers. ${ }^{92}$ Where stated, alumina flash chromatography employed Laporte Alumina, Grade H (100-200 mesh). Reversed phase flash chromatography employed. BakerBond Octadecyl $\mathrm{C}_{18}(40 \mu \mathrm{m})$ reversed phase packing, which was recycled by successive washings with DMSO, $\mathrm{CH}_{2} \mathrm{Cl}_{2}, \mathrm{MeOH}+1 \%$ TFA and $\mathrm{MeOH}$. Solvents used for chromatography were either purified by simple distillation (methanol) or from calcium hydride (ethyl acetate, dichloromethane and hexanes). Hexanes consisted of the fraction boiling at 50-70 ${ }^{\circ} \mathrm{C}$. Occasionally " $\mathrm{C}_{18}$ cartridges" were used for removal of salts from very polar compounds. These were Bakerbond spe ${ }^{\mathrm{TM}}$ Octadecyl $\left(\mathrm{C}_{18}\right)$ Disposable Extraction Columns, packed with 500 mg reversed phase octadecylsilane $\left(\mathrm{C}_{18}\right)$ bonded to silica gel ( $40 \mu \mathrm{m}$ APD, $60 \AA$ ). 


\section{Reagents and Solvents}

Reagents and solvents used in reactions were purified according to well-established procedures. ${ }^{124}$ Tetrahydrofuran, diethyl ether and benzene were distilled from sodium benzophenone ketyl immediately prior to use. Toluene and dichloromethane were distilled from calcium hydride. Acetic anhydride was distilled from phosphorus pentoxide. 1,2Dichloroethane was distilled from phosphorus pentoxide and stored over $4 \AA$ molecular sieves under an atmosphere of nitrogen. Acetyl chloride was distilled from quinoline $(10 \% \mathrm{v} / \mathrm{v})$ immediately prior to use. $N, N$-Dimethylformamide was sequentially dried over two batches of freshly activated $4 \AA$ molecular sieves $(2 \times 24 \mathrm{~h})$, before finally being stored under nitrogen over a third batch of $4 \AA$ molecular sieves. ${ }^{125}$ To remove residual dimethylamine, the solvent was evacuated $(\sim 0.1 \mathrm{~mm} \mathrm{Hg})$ for at least $15 \mathrm{~min}$ prior to use. Methanol was distilled from $\mathrm{Mg}(\mathrm{OMe})_{2}$ and stored under nitrogen. Diethyl carbonate was dried over calcium chloride and then distilled under argon, collecting the fraction boiling at $124^{\circ} \mathrm{C}$. Phosphorus oxychloride was fractionally distilled, collecting the fraction boiling at $106^{\circ} \mathrm{C}$. Solutions of $n-\mathrm{BuLi}$ in hexanes were titrated in diethyl ether with 2-butanol, using 1,10-phenanthroline as an indicator. ${ }^{126} p$ Bromoanisole was passed through a plug of neutral alumina immediately prior to use. Unless otherwise stated, $m$-CPBA was washed with $\mathrm{pH} 7.5$ buffer to remove $m$-chlorobenzoic acid. Concentrated aqueous ammonia solution was of analytical grade, and was stated to contain $\sim 25 \%$ ammonia, density $=0.91 \mathrm{~g} / \mathrm{mL}$.

Unless otherwise stated, all reactions were performed in oven- or flame-dried glassware under an atmosphere of dry argon or nitrogen. Reaction temperatures refer to the external bath temperature. Where the term "standard workup" or a similar phrase is used, aqueous phases were extracted with at least three portions of ethyl acetate or dichloromethane. The organic extracts were washed with brine and dried over $\mathrm{MgSO}_{4}$ or $\mathrm{Na}_{2} \mathrm{SO}_{4}$. Drying agents were filtered off, prior to concentration of solvents on a Büchi rotary evaporator. A high vacuum pump was used to remove any remaining solvent. 


\section{Notes on Nomenclature}

Due to the need for consistency and clarity of compound names, the naming of some compounds described in this dissertation deviates from IUPAC nomenclature in the following manner. For fused polycyclic compounds (with the exception of the ketone in 2.44 ), no characteristic groups have been cited as suffix (i.e. appointed as principal group). This in no way creates any ambiguity in the naming of compounds, and is in accord with the IUPAC recommendations in such a situation. ${ }^{127}$

For example, in this thesis, compound 4.9 is named

4-Methoxy-9-(4-methoxybenzylamino)-5-[2-(methylsulfanyl)pyrimidin-4-yl]pyrido[3',2';4,5]pyrrolo $[1,2-c] p y r i m i d i n e$

rather than IUPAC nomenclature

(4-Methoxybenzyl)-\{4-methoxy-5-[2-(methylsulfanyl)pyrimidin-4-yl]pyrido[3',2':4,5]pyrrolo[1,2-c] pyrimidin-9-yl\}amine.

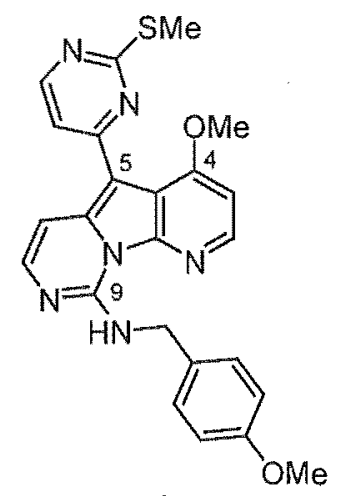




\section{Antitumour P388 Assay}

Samples were prepared for P388 assays by dissolving a known mass $(0.3-1.0 \mathrm{mg})$ in $\mathrm{MeOH}$ or $\mathrm{MeOH} / \mathrm{CH}_{2} \mathrm{Cl}_{2}$ (up to 1:3). In some cases, small amounts $(<1 \mu \mathrm{L}$ ) of TFA were added to increase solubility. Solvent blanks were also submitted in the assay as controls. A two-fold dilution series of the sample was incubated for $72 \mathrm{~h}$ with P388 (murine leukaemia) cells. The concentration of sample required to reduce the $\mathrm{P} 388$ cell growth by $50 \%$ (comparative to control cells) is determined using the absorbance values obtained when the yellow dye MTT tetrazolium is reduced by healthy cells to the purple MTT formazan (Figure 6.1). The result is expressed as an $\mathrm{IC}_{50}$ in $\mathrm{ng} / \mathrm{mL}$.
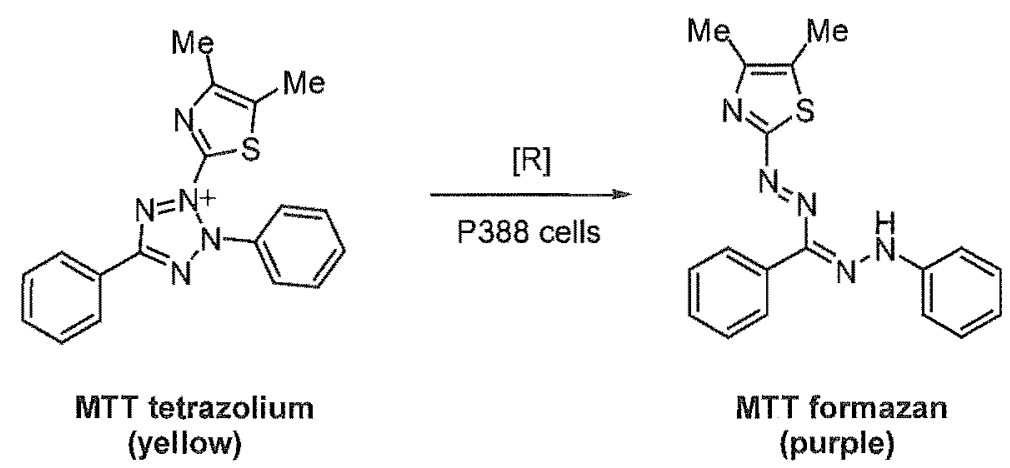

Figure 6.1 Reduction of MTT tetrazolium by viable cells of the P388 assay. 


\subsection{Experiments Described in Chapter Two}

4-Iodo-2-(methylsulfanyl)pyrimidine $2.12^{60}$

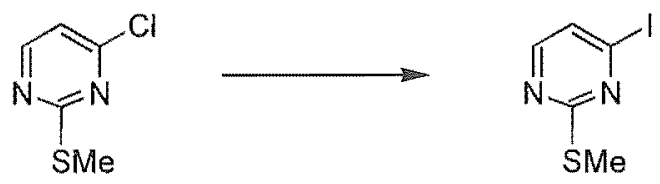

This was prepared from 4-chloro-2-(methylsulfanyl)pyrimidine $(2.13,14.3 \mathrm{~g}$ ), following the procedure of Undheim et al. ${ }^{60}$ To ensure the optimum yield, a magnetic flea of at least $50 \mathrm{~mm}$ was required for efficient stirring, and it was essential that large lumps were regularly broken up with a glass rod, to give a homogeneous brown paste. After isolation of the crude material, recrystallisation from hexanes gave pure iodide $\mathbf{2 . 1 2}$ as large white needles $(17.8 \mathrm{~g}, 79 \%)$.

Mp: $48-50^{\circ} \mathrm{C}$ (hexanes) $\left(\right.$ lit $^{60} 52-53^{\circ} \mathrm{C}$ ).

${ }^{1} \mathrm{H} \mathrm{NMR}\left(300 \mathrm{MHz}_{2} \mathrm{CDCl}_{3}\right): \delta 2.54\left(\mathrm{~s}, 3 \mathrm{H}, \mathrm{SCH}_{3}\right), 7.40(\mathrm{~d}, J=5.1 \mathrm{~Hz}, 1 \mathrm{H}), 8.00(\mathrm{~d}, J=5.1 \mathrm{~Hz}$, $1 \mathrm{H})$.

2-Chloronicotinoyl chloride $2.4^{61}$<smiles>O=C(O)c1cccnc1Cl</smiles><smiles>O=C(Cl)c1cccnc1Cl</smiles>

2-Chloronicotinic acid $(2.19,4.90 \mathrm{~g}, 31.1 \mathrm{mmol})$, thionyl chloride $(12 \mathrm{~mL}, 0.16 \mathrm{~mol})$ and dry DMF $(0.3 \mathrm{~mL}, 12 \mathrm{~mol} \%)$ were stirred at reflux for $26 \mathrm{~h}$, using a $\mathrm{CaCl}_{2}$ drying tube to exclude atmospheric moisture. After cooling, thionyl chloride was removed in vacuo, and the product was purified by Kugelrohr distillation $\left(110^{\circ} \mathrm{C}, 0.03 \mathrm{~mm} \mathrm{Hg}\right)$ to give acid chloride 2.4 as a white crystalline solid $(5.30 \mathrm{~g}, 97 \%)$. The solid was stored under argon and used within 1-2 days of distillation.

Mp: $38-41^{\circ} \mathrm{C}$, sweats at $34^{\circ} \mathrm{C}\left(1 \mathrm{lit}^{50} 39-44^{\circ} \mathrm{C}\right)$.

${ }^{1} \mathbb{H} \mathbb{N M R}\left(300 \mathrm{MHz}, \mathrm{CDCl}_{3}\right): \delta 7.45(\mathrm{dd}, J=4.6,7.9 \mathrm{~Hz}, 1 \mathrm{H}), 8.41(\mathrm{dd}, J=1.8,7.9 \mathrm{~Hz}, 1 \mathrm{H})$, $8.61(\mathrm{dd}, J=1.8,4.6 \mathrm{~Hz}, 1 \mathrm{H})$. 
(2-Chloropyridin-3-yl)bis-[2-(methylsulfanyl)pyrimidin-4-yl] methamol 2.3
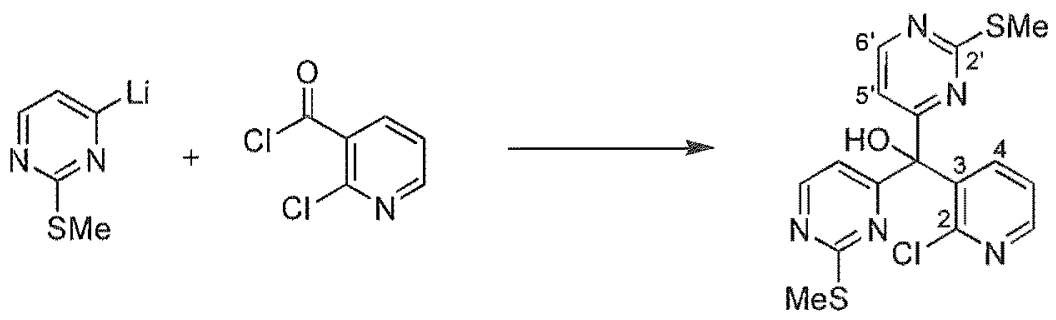

The reactor (Figure 2.8, p 41) was charged with iodide 2.12 (5.00 g, 19.8 mmol) and freshly distilled THF $(270 \mathrm{~mL})$, and cooled to $-95^{\circ} \mathrm{C}\left(\mathrm{MeOH} / \mathrm{N}_{2}\right)$. A solution of $n$-BuLi in hexanes $(1.57 \mathrm{M}, 12.6 \mathrm{~mL}, 19.8 \mathrm{mmol})$ was carefully added to the stirred solution over $20 \mathrm{~min}$, while maintaining the temperature at $-95^{\circ} \mathrm{C}$. After $20 \mathrm{~min}$, a solution of acid chloride $2.4(1.17 \mathrm{~g}, 6.67$ mmol) in THF $(10 \mathrm{~mL})$ was added to the dark mixture over $5 \mathrm{~min}$. After $3.5 \mathrm{~h}$ at $-95^{\circ} \mathrm{C}$, the reaction was quenched with methanol $(1 \mathrm{~mL})$ and allowed to warm overnight. Excess THF was removed in vacuo and the residue was taken up in water/EtOAc and worked up according to the standard procedure to give the crude material. Flash chromatography on silica, eluting with $25-$ $50 \%$ EtOAc/hexanes, gave triarylmethanol 2.3 (1.24 g, 93\% pure by ${ }^{1} \mathrm{H}$ NMR spectroscopy) in addition to a number of other side products, one of which was the biarylmethanol 2.23 . Fractions which contained mixtures of $\mathbf{2 . 3}$ and $\mathbf{2 . 2 3}$ were subjected to oxidation by $\mathrm{MnO}_{2}(2 \mathrm{~g})$ in refluxing benzene $(12 \mathrm{~mL})$. Filtration through celite (rinsing with boiling EtOAc) and concentration in vacuo gave a mixture of ketone $\mathbf{2 . 1 1}$ and the desired triarylmethanol 2.3, which was readily separated by chromatography to yield a further $343 \mathrm{mg}$ of $2.3\left(\sim 95 \%\right.$ pure by ${ }^{1} \mathrm{H}$ NMR spectroscopy). Thus, 2.3 was obtained as an orange foam (1.58 g, 93\% pure, $56 \%$ yield). An analytical sample was obtained by flash chromatography on reversed phase silica, eluting with $75 \% \mathrm{MeOH} /$ water, to give an off-white solid.

Mp: $54-58^{\circ} \mathrm{C}$.

${ }^{1} \mathrm{H}$ NMR $\left.\left(300 \mathrm{MHz}, \mathrm{CDCl}_{3}\right): \delta 2.49(\mathrm{~s}, 6 \mathrm{H}, \mathrm{SCH})_{3}\right), 6.33(\mathrm{~s}, 1 \mathrm{H}, \mathrm{OH}), 7.17(\mathrm{dd}, J=4.9,7.8 \mathrm{~Hz}$, $1 \mathrm{H}, \mathrm{H}-5$ ), 7.23 (dd, $J=2.0,7.8 \mathrm{~Hz}, 1 \mathrm{H}, \mathrm{H}-4), 7.40$ (d, $J=5.1 \mathrm{~Hz}, 2 \mathrm{H}, \mathrm{H}-5$ '), 8.38 (dd, $J=2.0$, $4.9 \mathrm{~Hz}, 1 \mathrm{H}, \mathrm{H}-6), 8.56$ (d, $\left.J=5.1 \mathrm{~Hz}, 2 \mathrm{H}, \mathrm{H}-6^{\prime}\right)$.

${ }^{13} \mathrm{C}$ NMR $\left(75 \mathrm{MHz}, \mathrm{CDCl}_{3}\right): \delta 14.2\left(\mathrm{SCH}_{3}\right), 79.4\left(\mathrm{Ar}_{3} \mathrm{COH}\right), 114.8\left(\mathrm{C}-5^{\prime}\right), 121.7(\mathrm{C}-5), 136.8$ (C-3), 138.9 (C-4), 149.1 (C-6), 151.5 (C-2), 158.0 (C-6'), 168.8 (C-4'), 171.8 (C-2').

$\mathbb{R}\left(\mathrm{CDCl}_{3}\right): \mathrm{v}_{\max } 3381,1549$. 
MS (EI): $m / z$ (rel intensity) 391/393 (59/25), 356 (100), 266/268 (71/29), 125 (68), 45 (53).

HRMS (EI): Calcd for $\mathrm{C}_{16} \mathrm{H}_{14}{ }^{35} \mathrm{ClN}_{5} \mathrm{O}^{32} \mathrm{~S}_{2}\left(\mathrm{M}^{+}\right) 391.0328$, found 391.0332 .

Other compounds isolated from this reaction and their spectroscopic data are listed below.

a) 2-(Methylsulfanyl)-4,6-bis[2-(methylsulfanyl)pyrimidin-4-yl]pyrimidine 2.22

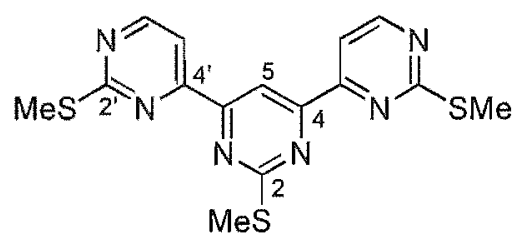

This compound was isolated as a tan solid.

Mp: dec at $236^{\circ} \mathrm{C}$.

${ }^{1} \mathrm{H}$ NMR $\left.\left(300 \mathrm{MHz}, \mathrm{CDCl}_{3}\right): \delta 2.69\left(\mathrm{~s}, 6 \mathrm{H}, 2^{\prime}-\mathrm{SCH}\right)_{3}\right), 2.72(\mathrm{~s}, 3 \mathrm{H}, 2-\mathrm{SCH}$ ), $8.05(\mathrm{~d}, J=5.1 \mathrm{~Hz}$, $\left.2 \mathrm{H}, \mathrm{H}-6^{\prime}\right), 8.73$ (d, $\left.J=5.1 \mathrm{~Hz}, 2 \mathrm{H}, \mathrm{H}-5^{\prime}\right), 9.08$ (s, $1 \mathrm{H}, \mathrm{H}-5$ ).

${ }^{13} \mathrm{C}$ NMR $\left(75 \mathrm{MHz}_{2} \mathrm{CDCl}_{3}\right): \delta 14.2\left(2^{\prime}-\mathrm{SCH}_{3}\right), 14.4\left(2-\mathrm{SCH}_{3}\right), 109.6(\mathrm{C}-5), 112.8\left(\mathrm{C}-5^{\prime}\right), 158.9$ (C-6'), 160.5 (C-4' or C-4), 162.7 (C-4 or C-4'), 173.1 (C-2'), $173.2(\mathrm{C}-2)$.

MS (EI): $m / z$ (rel intensity) 374 (100), 359 (16), 345 (15).

HRMS (EI): Calcd for $\mathrm{C}_{15} \mathrm{H}_{14} \mathrm{~N}_{6}{ }^{32} \mathrm{~S}_{3}\left(\mathrm{M}^{+}\right) 374.0442$, found 374.0452 .

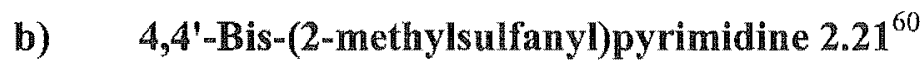

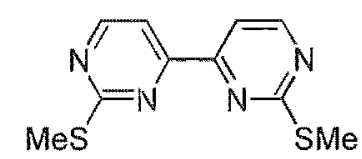

This compound was isolated as an off-white solid.

Mp: $187-189^{\circ} \mathrm{C}$, softens at $185^{\circ} \mathrm{C}\left(\mathrm{lit}^{60} 184-185^{\circ} \mathrm{C}\right)$.

${ }^{1}{ }_{\mathrm{H} \mathrm{NMR}}\left(500 \mathrm{MHz}, \mathrm{CDCl}_{3}\right): \delta 2.65(\mathrm{~s}, 6 \mathrm{H}), 8.04(\mathrm{~d}, J=5.1 \mathrm{~Hz}, 2 \mathrm{H}), 8.71(\mathrm{~d}, J=5.1 \mathrm{~Hz}, 2 \mathrm{H})$. 
c) (2-Chloropyridin-3-yl) $-[2-($ methylsullanyl)pyrimidin-4-yl]methamone 2.11

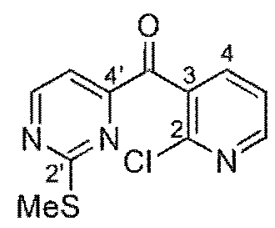

This compound was isolated as colourless blocks after recrystallisation from benzene/hexanes.

Mp: $107-109^{\circ} \mathrm{C}$ (benzene/hexanes).

${ }^{1} \mathrm{H}$ NMR $\left.\left(300 \mathrm{MHz}, \mathrm{CDCl}_{3}\right): \delta 2.37(\mathrm{~s}, 3 \mathrm{H}, \mathrm{SCH})_{3}\right), 7.40(\mathrm{dd}, J=4.9,7.6 \mathrm{~Hz}, 1 \mathrm{H}, \mathrm{H}-5), 7.64(\mathrm{~d}$, $\left.J=4.9 \mathrm{~Hz}, 1 \mathrm{H}, \mathrm{H}-5^{\prime}\right), 7.88(\mathrm{dd}, J=2.0,7.6 \mathrm{~Hz}, 1 \mathrm{H}, \mathrm{H}-4), 8.56(\mathrm{dd}, J=2.0,4.9 \mathrm{~Hz}, 1 \mathrm{H}, \mathrm{H}-6)$, $8.82\left(\mathrm{~d}, J=4.9 \mathrm{~Hz}, 1 \mathrm{H}, \mathrm{H}-6^{\prime}\right)$.

${ }^{13} \mathrm{C} \mathrm{NMR}\left(75 \mathrm{MHz}, \mathrm{CDCl}_{3}\right): \delta 14.0\left(\mathrm{SCH}_{3}\right), 113.4(\mathrm{C}-5), 122.0(\mathrm{C}-5), 133.3(\mathrm{C}-3), 139.1(\mathrm{C}-4)$, $148.5(\mathrm{C}-2), 151.4(\mathrm{C}-6), 158.6\left(\mathrm{C}-4^{\prime}\right), 159.4\left(\mathrm{C}-6^{\prime}\right), 173.6\left(\mathrm{C}-2^{\prime}\right), 192.8(\mathrm{C}=\mathrm{O})$.

$\mathbb{I R}(\mathrm{KBr}): \mathrm{v}_{\max } 1690,1556$.

MS (EI): $m / z$ (rel intensity) 267/269 (5/6), 230 (100), 140/142 (55/18), 112/114 (39/13).

HRMS (EI): Calcd for $\mathrm{C}_{11} \mathrm{H}_{8}{ }^{35} \mathrm{CIN}_{3} \mathrm{O}^{32} \mathrm{~S}\left(\mathrm{M}^{+}\right) 265.0077$ found 265.0072 .

d) (2-Chloropyridin-3-yl)-[2-(methylsulfanyl)pyrimidin-4-yl]methanol 2.23<smiles>CS(=O)(=O)c1nccc(C(O)c2cccnc2Cl)n1</smiles>

This compound was isolated as a viscous gum.

${ }^{1} \mathrm{H}$ NMR $\left(500 \mathrm{MHz}, \mathrm{CDCl}_{3}\right): \delta 2.58(\mathrm{~s}, 3 \mathrm{H}, \mathrm{SCH}), 4.88(\mathrm{~d}, J=3.9 \mathrm{~Hz}, 1 \mathrm{H}, \mathrm{OH}), 6.13(\mathrm{~d}, J=$ $3.9 \mathrm{~Hz}, 1 \mathrm{H}, \mathrm{Ar}_{2} \mathrm{CHOH}$ ), 7.00 (d, $J=5.1 \mathrm{~Hz}, 1 \mathrm{H}, \mathrm{H}-5$ ), 7.28 (dd, $J=4.8,7.8 \mathrm{~Hz}, 1 \mathrm{H}, \mathrm{H}-5$ ), 7.80 (dd, $J=1.8,7.8 \mathrm{~Hz}, 1 \mathrm{H}, \mathrm{H}-4), 8.36$ (dd, $J=1.8,4.8 \mathrm{~Hz}, 1 \mathrm{H}, \mathrm{H}-6$ ), 8.46 (d, $J=5.1 \mathrm{~Hz}, 1 \mathrm{H}, \mathrm{H}-6$ ). ${ }^{13} \mathrm{C} \mathrm{NMR}\left(75 \mathrm{MHz}, \mathrm{CDCl}_{3}\right): \delta 14.0\left(\mathrm{SCH}_{3}\right), 70.5\left(\mathrm{Ar}_{2} \mathrm{CHOH}\right), 113.0(\mathrm{C}-5), 123.0(\mathrm{C}-5), 136.0$ (C-3), 137.7 (C-4), 148.9 (C-6), 149.5 (C-2), 157.6 (C-6'), 168.0 (C-4'), 172.3 (C-2').

IR $\left(\mathrm{CDCl}_{3}\right): \mathrm{v}_{\max } 3416,1562$. 
MS (EI): $m / z$ (rel intensity) $267 / 269$ (6/2), 265 (10), 232 (83), $231(61), 230(100), 216(39), 214$ (30), 140/142 (84/50), $125(42), 112 / 114(80 / 34), 76(46)$.

HRMS (EI): Calcd for $\mathrm{C}_{11} \mathrm{H}_{10} \mathrm{~N}_{3} \mathrm{O}^{32} \mathrm{~S}\left(\mathrm{M}^{+}-\mathrm{Cl}\right) 232.0545$, found 232.0542 .

\section{9-(Methylsulfanyl)-5-[2-(methylsulfanyl)pyrimidin-4-yl]pyrido $\left[3^{\prime}, 2^{\prime}: 4,5\right]$ pyrrolo[1,2-c]} pyrimidine 2.1
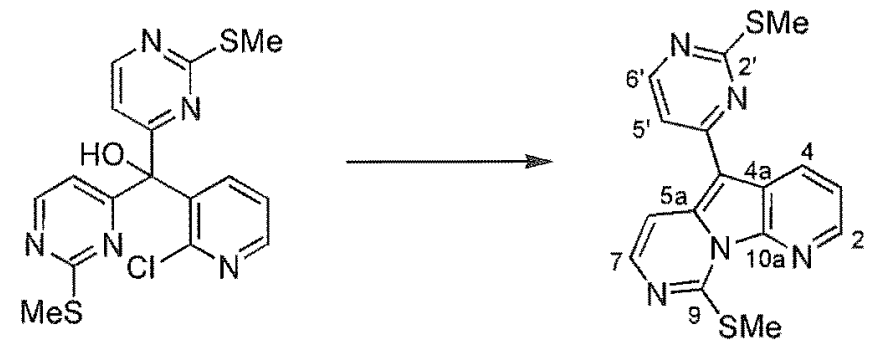

Triarylmethanol 2.3 (97\% pure, $497 \mathrm{mg}, 1.23 \mathrm{mmol})$ was heated with TFA $(0.41 \mathrm{~mL}, 5.3 \mathrm{mmol})$ and TES $(1.65 \mathrm{~mL}, 10.3 \mathrm{mmol})$ at $75^{\circ} \mathrm{C}$ for $66 \mathrm{~h}$. After concentration of excess reagents in vacuo the residue was dissolved in $\mathrm{CH}_{2} \mathrm{Cl}_{2}(65 \mathrm{~mL})$ and neutralised with $5 \%$ aq $\mathrm{NaHCO}_{3}$ solution $(40 \mathrm{~mL})$. After extraction with $\mathrm{CH}_{2} \mathrm{Cl}_{2}$ the extracts were worked up in the usual manner. Analysis of the ${ }^{1} \mathrm{H}$ NMR spectrum of the crude mixture revealed the presence of the title compound, and lesser amounts of $\mathbf{2 . 3 9}$ and $\mathbf{2 . 4 2}$, in addition to several unidentified products. Repeated flash chromatography on silica, eluting with 37-70\% EtOAc/hexanes, gave the variolin core 2.1 as a yellow solid (143 $\mathrm{mg}, 34 \%)$.

Mp: $210-211^{\circ} \mathrm{C}(\mathrm{MeOH} / 1,2-$ dichloroethane $)$.

${ }^{1} \mathrm{H}$ NMR $\left(500 \mathrm{MHz}, \mathrm{CDCl}_{3}\right): \delta 2.68\left(\mathrm{~s}, 3 \mathrm{H}, 2^{\prime}-\mathrm{SCH} H_{3}\right), 2.74(\mathrm{~s}, 3 \mathrm{H}, 9-\mathrm{SCH}), 7.36(\mathrm{~d}, J=5.1 \mathrm{~Hz}$, $\left.1 \mathrm{H}, \mathrm{H}-5^{\prime}\right), 7.52(\mathrm{dd}, J=4.5,8.2 \mathrm{~Hz}, 1 \mathrm{H}, \mathrm{H}-3), 7.83$ (d, $\left.J=6.8 \mathrm{~Hz}, 1 \mathrm{H}, \mathrm{H}-7\right), 8.07$ (d, $J=6.8 \mathrm{~Hz}$, $1 \mathrm{H}, \mathrm{H}-6), 8.52(\mathrm{~d}, J=5.1 \mathrm{~Hz}, 1 \mathrm{H}, \mathrm{H}-6), 8.61$ (dd, $J=1.5,4.5 \mathrm{~Hz}, 1 \mathrm{H}, \mathrm{H}-2), 8.65(\mathrm{dd}, J=1.5$, $8.2 \mathrm{~Hz}, 1 \mathrm{H}, \mathrm{H}-4)$.

${ }^{13} \mathrm{C}$ NMR $\left(75 \mathrm{MHz} \mathrm{CDCl}_{3}\right): \delta 14.3\left(2^{\prime}-\mathrm{SCH}_{3}\right), 14.9\left(9-\mathrm{SCH}_{3}\right), 101.4(\mathrm{C}-5), 108.3(\mathrm{C}-6), 112.9$ $\left(\mathrm{C}^{\prime} 5^{\prime}\right), 120.7(\mathrm{C}-4 \mathrm{a}), 120.8(\mathrm{C}-3), 128.0(\mathrm{C}-4), 137.5$ (C-5a), 139.9 (C-7), $141.8(\mathrm{C}-2), 143.0(\mathrm{C}-$ 10a), 155.1 (C-9), $156.6\left(\mathrm{C}^{\prime} 6^{\prime}\right), 160.9\left(\mathrm{C}-4^{\prime}\right), 172.4\left(\mathrm{C}-2 '^{\prime}\right)$.

MS (EI): $m / z$ (rel intensity) $339(100), 306(10), 220(14), 133$ (12),

HIRMS (EI): Calcd for $\mathrm{C}_{16} \mathrm{H}_{13} \mathrm{~N}_{5}{ }^{32} \mathrm{~S}_{2}\left(\mathrm{M}^{+}\right) 339.0612$, found 339.0617 . 
Other compounds isolated from this reaction and their spectroscopic data are listed below (from least to greatest polarity).

a) 9-(Methylsulfanyl)-5-[2-(methylsulfanyl)pyrimidin-4-yloxy]pyrido[3',2':4,5]pyrrolo $[1,2-c]$ pyrimidine 2.42<smiles>Cc1nccc(Oc2c3cccnc3n3c(C)nccc23)n1</smiles>

This could be further purified by reversed phase preparative HPLC, eluting with $85 \%$ $\mathrm{CH}_{3} \mathrm{CN} /$ water $+0.05 \% \mathrm{TFA}$, to give a yellow solid.

Mp: $205-207^{\circ} \mathrm{C}$, sweats at $203^{\circ} \mathrm{C}(\mathrm{MeOH} /$ water).

${ }^{1} \mathrm{H}$ NMR $\left(300 \mathrm{MHz}_{2} \mathrm{CDCl}_{3}\right): \delta 2.21\left(\mathrm{~s}, 3 \mathrm{H}, 2{ }^{\prime}-\mathrm{SCH} H_{3}\right), 2.72\left(\mathrm{~s}, 3 \mathrm{H}, 9-\mathrm{SCH}{ }_{3}\right), 6.62(\mathrm{~d}, J=5.4 \mathrm{~Hz}$, $\left.1 \mathrm{H}, \mathrm{H}-5^{\prime}\right), 6.96$ (d, $\left.J=6.6 \mathrm{~Hz}, 1 \mathrm{H}, \mathrm{H}-6\right), 7.39$ (dd, $\left.J=4.8,8.2 \mathrm{~Hz}, 1 \mathrm{H}, \mathrm{H}-3\right), 7.49$ (d, $J=6.6 \mathrm{~Hz}$, $1 \mathrm{H}, \mathrm{H}-7), 7.85$ (dd, $J=1.5,8.2 \mathrm{~Hz}, 1 \mathrm{H}, \mathrm{H}-4), 8.39$ (d, $J=5.4 \mathrm{~Hz}, 1 \mathrm{H}, \mathrm{H}-6$ ), 8.56 (dd, $J=1.5$, $4.8 \mathrm{~Hz}, 1 \mathrm{H}, \mathrm{H}-2)$.

${ }^{13} \mathrm{C} \mathrm{NMR}\left(75 \mathrm{MHz}, \mathrm{CDCl}_{3}\right): \delta 13.9\left(2^{\prime}-\mathrm{SCH}_{3}\right), 14.6\left(9-\mathrm{SCH}_{3}\right), 102.2\left(\mathrm{C}-5^{\prime}\right), 104.8(\mathrm{C}-6), 115.6$ (C-4a), 119.0 (C-5), 119.7 (C-3), 125.4 (C-4), 126.2 (C-5a), 135.4 (C-7), 139.0 (C-10a), 141.5 (C-2), 153.5 (C-9), 158.8 (C-6'), 168.5 (C-4'), 173.2 (C-2').

MS (EI): $m / z$ (rel intensity) 355 (94), 308 (17), 230 (100), 143 (19), 113 (18), 73 (15).

HRMS (EI): Calcd for $\mathrm{C}_{16} \mathrm{H}_{13} \mathrm{~N}_{5} \mathrm{O}^{32} \mathrm{~S}_{2}\left(\mathrm{M}^{+}\right) 355.0562$, found 355.0559 . 
b) (2-Chloropyridin-3-yl)-[2-(methylsulfanyl)pyrimidin-4-yl]-[2-(methylsulfanyl) pyrimidin-4-yloxy]methane 2.39

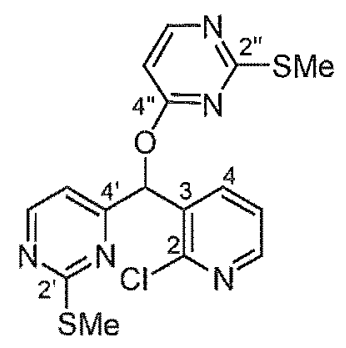

This could be further purified by flash chromatography on reversed phase silica, eluting with $80 \% \mathrm{MeOH} / \mathrm{water}$, followed by flash chromatography on silica, eluting with $50 \%$ EtOAc/hexanes, to give a viscous gum.

${ }^{1} \mathrm{H}$ NMR $\left(300 \mathrm{MHz}, \mathrm{CDCl}_{3}\right.$ ): $\delta 2.43$ (s, 3H, 2"-SCH $), 2.44$ (s, 3H, 2'-SCH ), 6.58 (d, $J=5.9$ $\mathrm{Hz}, 1 \mathrm{H}, \mathrm{H}-5 "), 7.19$ (d, $\left.J=4.9 \mathrm{~Hz}, 1 \mathrm{H}, \mathrm{H}-5^{\prime}\right), 7.27$ (dd, $J=4.8,7.6 \mathrm{~Hz}, 1 \mathrm{H}, \mathrm{H}-5$ ), 7.43 (s, $1 \mathrm{H}$, $\mathrm{Ar}_{2} \mathrm{CHOAr}$ ), 7.84 (dd, $J=1.8,7.6 \mathrm{~Hz}, 1 \mathrm{H}, \mathrm{H}-4$ ), 8.31 (d, $J=5.9 \mathrm{~Hz}, 1 \mathrm{H}, \mathrm{H}-6^{\prime \prime}$ ), 8.38 (dd, $J=$ $1.8,4.8 \mathrm{~Hz}, 1 \mathrm{H}, \mathrm{H}-6), 8.54$ (d, $J=4.9 \mathrm{~Hz}, 1 \mathrm{H}, \mathrm{H}-6^{\prime}$ ).

${ }^{13} \mathrm{C} \mathrm{NMR}\left(75 \mathrm{MHz}, \mathrm{CDCl}_{3}\right): 813.91\left(\mathrm{SCH}_{3}\right), 13.93\left(\mathrm{SCH}_{3}\right), 74.3\left(\mathrm{Ar}_{2} \mathrm{CHOAr}\right), 103.2(\mathrm{C}-5 ")$, 113.4 (C-5'), 122.6 (C-5), 132.5 (C-3), 137.6 (C-4), 149.3 (C-6), 150.1 (C-2), 157.8 (C-6'), 158.0 (C-6"), $165.3\left(\mathrm{C}-4^{\prime}\right), 166.8\left(\mathrm{C}-4^{\prime \prime}\right), 172.3\left(\mathrm{C}-2^{\prime \prime}\right), 172.9\left(\mathrm{C}-2^{\prime}\right)$.

MS (ES): $m / z$ (rel intensity) 392/394 (100/40), 356 (19), 250/252 (39/18).

HRMS (ES): Calcd for $\mathrm{C}_{16} \mathrm{H}_{15}{ }^{35} \mathrm{ClN}_{5} \mathrm{O}^{32} \mathrm{~S}_{2}\left(\mathrm{MH}^{+}\right) 392.0407$, found 392.0409 .

9-(Methylsulfanyl)-5a-[2-(methylsulfanyl)pyrimidin-4-yl]-5all-pyrido[ $\left.3^{\prime}, 2^{\prime}: 4,5\right]$ pyrrolo [1,2-c]pyrimidin-5-one 2.44
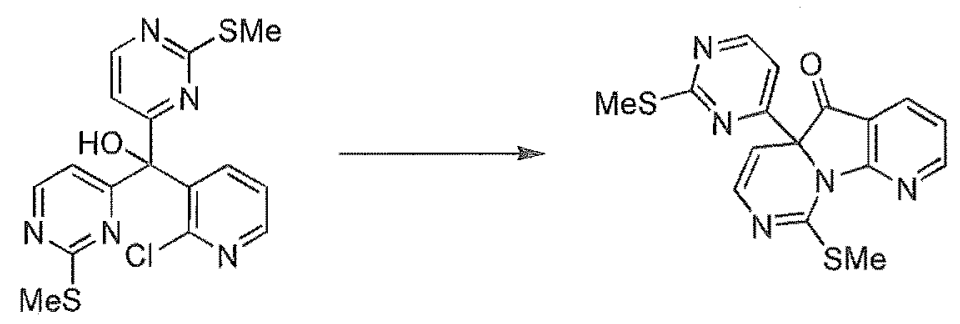

Triarylmethanol $2.3(500 \mathrm{mg}, 1.28 \mathrm{mmol})$ was heated with TFA $(0.82 \mathrm{~mL}, 11 \mathrm{mmol})$ at $80^{\circ} \mathrm{C}$ for $10 \mathrm{~h}$. After removal of excess TFA in vacuo, the residue was dissolved in $\mathrm{CH}_{2} \mathrm{Cl}_{2}(65 \mathrm{~mL})$ and 
neutralised with $5 \%$ aq $\mathrm{NaHCO}_{3}$ solution ( $40 \mathrm{~mL}$ ). After extraction with $\mathrm{CH}_{2} \mathrm{Cl}_{2}$ the extracts were worked up as usual. ${ }^{1} \mathrm{H}$ NMR analysis of the crude material showed it to contain $\mathbf{2 . 4 4}$ as the major product, together with lesser amounts of 2.39, 2.42 and 2.11. Flash chromatography on silica, eluting with 30-50\% EtOAc/hexanes, followed by flash chromatography on reversed phase silica, eluting with $75 \% \mathrm{MeOH} /$ water, gave ketone $2.44\left(\sim 95 \%\right.$ pure by ${ }^{1} \mathrm{H} \mathrm{NMR}, 70 \mathrm{mg}$, $15 \%$ ). An analytical sample was obtained by reversed phase preparative HPLC, eluting with $75 \% \mathrm{CH}_{3} \mathrm{CN} /$ water $+0.05 \% \mathrm{TFA}$, to give a yellow solid. This compound slowly decomposes over a matter of weeks at room temperature.

Mp: $52-55^{\circ} \mathrm{C}$.

${ }^{1} \mathrm{H}$ NMR $\left(500 \mathrm{MHz}, \mathrm{CDCl}_{3}\right): \delta 2.38\left(\mathrm{~s}, 3 \mathrm{H}, 2^{\prime}-\mathrm{SCH}_{3}\right), 2.52(\mathrm{~s}, 3 \mathrm{H}, 9-\mathrm{SCH}$ ), $5.96(\mathrm{~d}, J=6.6 \mathrm{~Hz}$, $1 \mathrm{H}, \mathrm{H}-6), 6.76(\mathrm{~d}, J=6.6 \mathrm{~Hz}, 1 \mathrm{H}, \mathrm{H}-7), 7.12(\mathrm{dd}, J=4.9,7.4 \mathrm{~Hz}, 1 \mathrm{H}, \mathrm{H}-3), 7.14$ (d, $J=4.9 \mathrm{~Hz}$, $\left.1 \mathrm{H}, \mathrm{H}-5^{\prime}\right), 7.96$ (dd, $\left.J=1.6,7.4 \mathrm{~Hz}, 1 \mathrm{H}, \mathrm{H}-4\right), 8.47$ (d, $J=4.9 \mathrm{~Hz}, 1 \mathrm{H}, \mathrm{H}-6$ ), 8.66 (dd, $J=1.6$, $4.9 \mathrm{~Hz}, 1 \mathrm{H}, \mathrm{H}-2)$.

${ }^{13} \mathrm{C} \mathrm{NMR}\left(75 \mathrm{MHz}, \mathrm{CDCl}_{3}\right): \delta 14.08\left(\mathrm{SCH}_{3}\right), 14.14\left(\mathrm{SCH}_{3}\right), 70.8(\mathrm{C}-5 \mathrm{a}), 107.1(\mathrm{C}-6), 112.66$ $\left(\mathrm{C}-5^{\prime}\right), 112.71(\mathrm{C}-4 \mathrm{a}), 118.8$ (C-3), 134.6 (C-4), 136.9 (C-7), 154.6 (C-9), 156.2 (C-2), 158.1 (C6'), 164.3 (C-10a), 165.4 (C-4'), 172.8 (C-2'), 193.2 (C-5).

$\mathbb{I R}\left(\mathrm{CDCl}_{3}\right): v_{\max } 1732,1414$.

HRMS (ES): Calcd for $\mathrm{C}_{16} \mathrm{H}_{14} \mathrm{~N}_{5} \mathrm{O}^{32} \mathrm{~S}_{2}\left(\mathrm{MH}^{+}\right) 356.0640$, found 356.0642 .

(2-Chloropyridin-3-yl)-(4-methoxyphenyl)-[2-(methylsulfanyl)pyrimidin-4-yl] methanol 2.49<smiles>CSc1nccc(C(=O)c2cccnc2Cl)n1</smiles><smiles>COc1ccc(Cl)cc1</smiles><smiles>CI</smiles><smiles>COc1ccc(C(O)(c2cccnc2Cl)c2cccnc2SC)cc1</smiles>

A solution of $n$-BuLi in hexanes $(1.6 \mathrm{M}, 85 \mu \mathrm{L}, 0.14 \mathrm{mmol})$ was added dropwise to a stirred solution of $p$-bromoanisole $(17 \mu \mathrm{L}, 0.14 \mathrm{mmol})$ in freshly distilled THF $(1.4 \mathrm{~mL})$ at $-78{ }^{\circ} \mathrm{C}$ (acetone/ $\left.\mathrm{CO}_{2}\right)$. After $20 \mathrm{~min}$ at $-78^{\circ} \mathrm{C}$ the reaction was cooled to $-97^{\circ} \mathrm{C}\left(\mathrm{MeOH} / \mathrm{N}_{2}\right)$ and a 
similarly cooled solution of $2.11(36 \mathrm{mg}, 0.14 \mathrm{mmol})$ in THF $(1.5 \mathrm{~mL})$ was added. After $0.5 \mathrm{~h}$ at $-97^{\circ} \mathrm{C}$, the reaction was allowed to warm to $\mathrm{rt}$ over $\sim 3 \mathrm{~h}$. The mixture was shaken with sat aq $\mathrm{NH}_{4} \mathrm{Cl}$ solution and worked up with EtOAc in the usual manner. Flash chromatography on silica, eluting with $45 \%$ EtOAc/hexanes, gave alcohol $\mathbf{2 . 4 9}$ as a pale yellow gum (23 mg, 45\%).

${ }^{1} \mathrm{H}$ NMR $\left(300 \mathrm{MHz}, \mathrm{CDCl}_{3}\right): \delta 2.48(\mathrm{~s}, 3 \mathrm{H}), 3.82(\mathrm{~s}, 3 \mathrm{H}), 5.32(\mathrm{~s}, 1 \mathrm{H}), 6.88-6.91(\mathrm{~m}, 2 \mathrm{H}), 6.95$ $(\mathrm{d}, J=5.1 \mathrm{~Hz}, 1 \mathrm{H}), 7.15(\mathrm{dd}, J=4.4,7.8 \mathrm{~Hz}, 1 \mathrm{H}), 7.29-7.33(\mathrm{~m}, 3 \mathrm{H}), 8.35(\mathrm{dd}, J=2.0,4.4 \mathrm{~Hz}$, $1 \mathrm{H}), 8.50(\mathrm{~d}, J=5.1 \mathrm{~Hz}, 1 \mathrm{H})$.

$\mathbb{I R}\left(\mathrm{CDCl}_{3}\right): \mathrm{v}_{\max } 3420,1560$.

MS (EI): $m / z$ (rel intensity) 373/375 (96/38), 355/357 (47/25), 340 (33), 338 (37), 322 (53), 321

(72), 294 (30), 261 (30), 248/250 (100/37), 140 (50), 135 (76), 125 (48), 77 (28).

HRMS (EI): Calcd for $\mathrm{C}_{18} \mathrm{H}_{16}{ }^{35} \mathrm{ClN}_{3} \mathrm{O}_{2}{ }^{32} \mathrm{~S}\left(\mathrm{M}^{+}\right)$373.0652, found 373.0660 .

5-(2-Chloropyridin-3-yl)-8-methoxy-1-(methylsulfanyl)pyrimido[1,6-a]indole 2.52
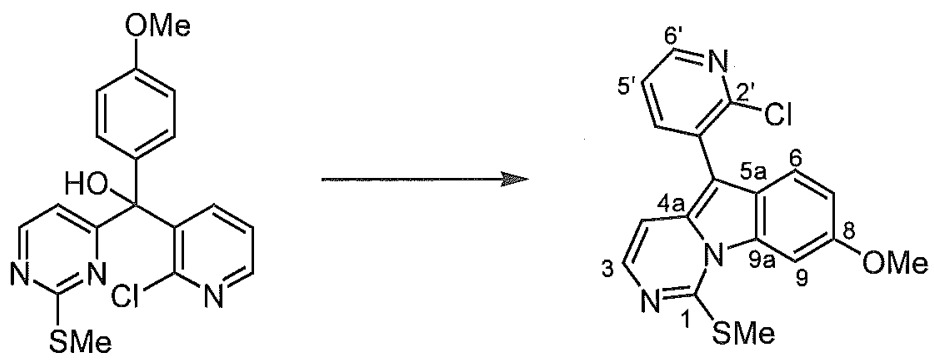

Alcohol 2.49 (12 mg, $0.032 \mathrm{mmol})$ was dissolved in TFA $(0.5 \mathrm{~mL})$, producing a yellow-green solution. TES $(6 \mu \mathrm{L}, 0.04 \mathrm{mmol})$ was added and the mixture was stirred at $70{ }^{\circ} \mathrm{C}$ for $24 \mathrm{~h}$. Further portions of TFA $(0.5 \mathrm{~mL})$ were added after 3 and $20 \mathrm{~h}$. After neutralisation with sat aq $\mathrm{NaHCO}_{3}$ solution and standard workup (EtOAc), the crude material was purified by flash chromatography on silica, eluting with $35 \%$ EtOAc/hexanes, to give the title compound $\mathbf{2 . 5} 2$ as a yellow-green solid (6 mg, 53\%). Recrystallisation by slow evaporation of a hexanes solution gave pale green needles suitable for $\mathrm{X}$-ray diffraction analysis.

Mp: $136-137^{\circ} \mathrm{C}$ (hexanes).

${ }^{1} \mathrm{H} \mathbb{N M R}\left(300 \mathrm{MHz}, \mathrm{CDCl}_{3}\right): \delta 2.82\left(\mathrm{~s}, 3 \mathrm{H}, \mathrm{SCH}_{3}\right), 3.98\left(\mathrm{~s}, 3 \mathrm{H}, \mathrm{OCH}_{3}\right), 6.86(\mathrm{~d}, J=6.6 \mathrm{~Hz}, 1 \mathrm{H}$, $\mathrm{H}-4), 7.15$ (dd, $J=2.3,9.0 \mathrm{~Hz}, 1 \mathrm{H}, \mathrm{H}-7), 7.37$ (dd, $J=4.6,7.6 \mathrm{~Hz}, 1 \mathrm{H}, \mathrm{H}-5$ ') 7.44 (d, $J=6.6$ 
$\mathrm{Hz}, 1 \mathrm{H}, \mathrm{H}-3), 7.49(\mathrm{~d}, J=9.0 \mathrm{~Hz}, 1 \mathrm{H}, \mathrm{H}-6), 7.83(\mathrm{dd}, J=2.0,7.6 \mathrm{~Hz}, 1 \mathrm{H}, \mathrm{H}-4), 8.26(\mathrm{~d}, J=2.3$ $\mathrm{Hz}, 1 \mathrm{H}, \mathrm{H}-9), 8.47\left(\mathrm{dd}, J=2.0,4.6 \mathrm{~Hz}, 1 \mathrm{H}, \mathrm{H}-6^{\prime}\right)$.

${ }^{13} \mathrm{C} \mathrm{NMR}\left(75 \mathrm{MHz}, \mathrm{CDCl}_{3}\right): \delta 14.7\left(\mathrm{SCH}_{3}\right), 55.9\left(\mathrm{OCH}_{3}\right), 100.1(\mathrm{C}-9), 103.5(\mathrm{C}-5), 107.8(\mathrm{C}-$ 4), $114.4(\mathrm{C}-7), 119.3(\mathrm{C}-6), 122.4\left(\mathrm{C}-5^{\prime}\right), 123.5$ (C-5a), $129.3\left(\mathrm{C}-3^{\prime}\right), 130.0(\mathrm{C}-9 \mathrm{a}), 133.3(\mathrm{C}-4 \mathrm{a})$, 134.1 (C-3), $141.4\left(\mathrm{C}^{\prime}\right), 148.4$ (C-6'), 151.6 (C-2'), 153.2 (C-1), 155.5 (C-8).

MS (EI): $m / z$ (rel intensity) 355/357 (100/37), $340 / 342$ (69/26).

HRMS (EI): Calcd for $\mathrm{C}_{18} \mathrm{H}_{14}{ }^{35} \mathrm{ClN}_{3} \mathrm{O}^{32} \mathrm{~S}\left(\mathrm{M}^{+}\right)$355.0546, found 355.0536 .

Crystal data and structure refinement for 2.52 :

Empirical formula

Formula weight

Temperature

Wavelength

Crystal system

Space group

Unit cell dimensions

Volume

Z

Density (calculated)

Absorption coefficient

$\mathrm{F}(000)$

Crystal size

Theta range for data collection

Index ranges

Reflections collected

Independent reflections

Completeness to theta $=22.48^{\circ}$

Refinement method

Data / restraints / parameters

Goodness-of-fit on $\mathrm{F}^{2}$

Final $R$ indices [ $1>2$ sigma(I)]

$\mathrm{R}$ indices (all data)

Largest diff. peak and hole
$\mathrm{C}_{18} \mathrm{H}_{14} \mathrm{ClN}_{3} \mathrm{OS}$

355.83

169(2) K

$0.71073 \AA$

Rhombohedral

R-3

$\begin{array}{ll}\mathrm{a}=34.678(7) \AA & \alpha=90^{\circ} . \\ \mathrm{b}=34.678(7) \AA & \beta=90^{\circ} . \\ \mathrm{c}=7.510(2) \AA & \gamma=120^{\circ} .\end{array}$

$7821(3) \AA^{3}$

18

$1.360 \mathrm{Mg} / \mathrm{m}^{3}$

$0.349 \mathrm{~mm}^{-1}$

3312

$0.90 \times 0.08 \times 0.07 \mathrm{~mm}^{3}$

2.03 to $22.48^{\circ}$.

$-37 \leq \mathrm{h} \leq 37,-37 \leq \mathrm{k} \leq 37,-8 \leq 1 \leq 4$

24735

$2268[\mathrm{R}(\mathrm{int})=0.1483]$

$100.0 \%$

Full-matrix least-squares on $\mathrm{F}^{2}$

$2268 / 0 / 254$

1.160

$\mathrm{R} 1=0.1317, \mathrm{wR} 2=0.3154$

$\mathrm{R} 1=0.1664, \mathrm{wR} 2=0.3406$

1.654 and -0.660 e. $\AA^{-3}$ 


\section{5-[2-(Methylsulfanyl)pyrimidin-4-yl]-9-(1-pyrrolidinyl)pyrido o[3',2':4,5]pyrolo[1,2-c]} pyrimidine 2.66
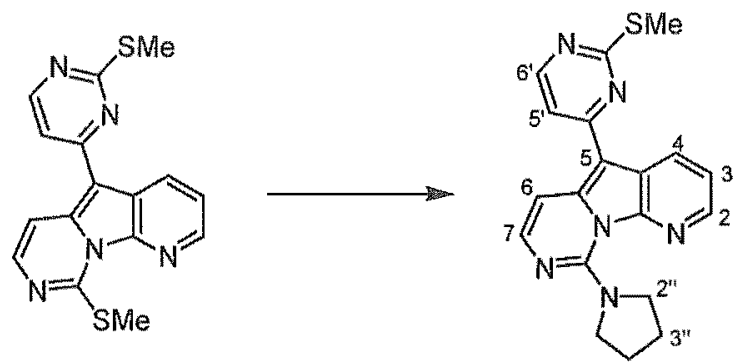

A mixture of $2.1(20 \mathrm{mg}, 0.059 \mathrm{mmol})$, and pyrrolidine $(50 \mu \mathrm{L}, 0.60 \mathrm{mmol})$ were heated in dioxane $(0.5 \mathrm{~mL})$ at $80^{\circ} \mathrm{C}$. A further portion of pyrrolidine $(50 \mu \mathrm{L}, 0.60 \mathrm{mmol})$ was added after $15 \mathrm{~h}$. After $27 \mathrm{~h}$, the reaction mixture was concentrated in vacuo, and the residue was purified by flash chromatography on silica, eluting with $55-75 \%$ EtOAc/hexanes, to give amine 2.66 as a yellow solid (11 mg, 51\%).

${ }^{1} \mathrm{H} \operatorname{NMR}\left(500 \mathrm{MHz}, \mathrm{CDCl}_{3}\right): \delta 1.98-2.01(\mathrm{~m}, 4 \mathrm{H}, \mathrm{H}-3 "), 2.65(\mathrm{~s}, 3 \mathrm{H}, \mathrm{SCH}), 3.82(\mathrm{t}, J=6.6 \mathrm{~Hz}$, $\left.4 \mathrm{H}, \mathrm{H}-2^{\prime}\right), 7.27$ (d, $\left.J=5.4 \mathrm{~Hz}, 1 \mathrm{H}, \mathrm{H}-5^{\prime}\right), 7.38$ (dd, $J=4.6,7.9 \mathrm{~Hz}, 1 \mathrm{H}, \mathrm{H}-3$ ), 7.58-7.61 (m, 2H, $\mathrm{H}-6, \mathrm{H}-7), 8.42-8.44$ (m, 2H, H-2, H-6'), 8.62 (dd, $J=1.5,7.9 \mathrm{~Hz}, 1 \mathrm{H}, \mathrm{H}-4$ ).

${ }^{13} \mathrm{C}$ NMR $\left(75 \mathrm{MHz}, \mathrm{CDCl}_{3}\right): \delta 14.2\left(\mathrm{SCH}_{3}\right), 25.5(\mathrm{C}-3 "), 51.3(\mathrm{C}-2 "), 100.5(\mathrm{C}-5), 103.5(\mathrm{C}-6)$, 112.9 (C-5'), 120.1 (C-3), 121.7 (C-4a), 127.6 (C-4), 140.9 (C-2), 141.8 (C-5a), 142.2 (C-7), 143.6 (C-10a), 149.9 (C-9), 156.3 (C-6'), 161.3 (C-4'), 172.2 (C-2').

9-(Methylsulfanyl)-5-[2-(methylsulfinyl)pyrimidin-4-yl]pyrido[3',2':4,5]pyrrolo[1,2-c] pyrimidine 2.67
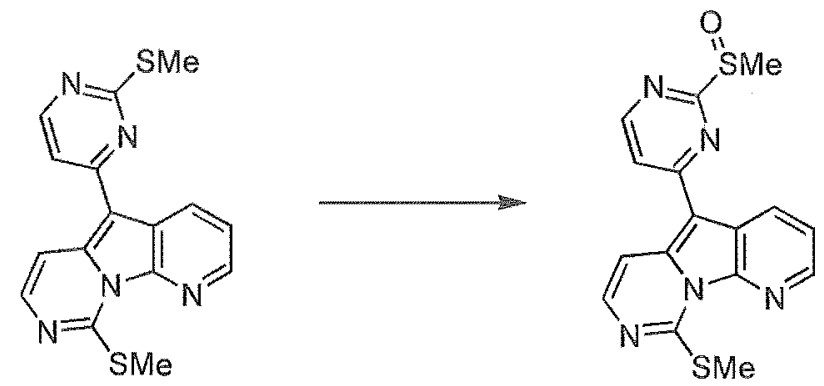

A solution of Oxone $\mathrm{e}^{\circledR}(55 \mathrm{mg}, 0.18 \mathrm{mmol})$ in water $(1 \mathrm{~mL})$ was added dropwise to a solution of 2.1 (10.0 mg, $29.5 \mu \mathrm{mol})$ in freshly distilled THF $(3 \mathrm{~mL})$ at rt. After $40 \mathrm{~min}$ the reaction was 
quenched with sat aq $\mathrm{NaHCO}_{3}$ solution and worked up in the usual manner with $\mathrm{CH}_{2} \mathrm{Cl}_{2}$ to give $7.9 \mathrm{mg}$ of crude material. Flash chromatography on silica, eluting with 5-10\% $\mathrm{MeOH} / \mathrm{EtOAc}$, gave mono-sulfoxide 2.67 as a yellow gum $(2.5 \mathrm{mg}, 24 \%)$.

${ }^{1} \mathrm{H}$ NMR $\left(500 \mathrm{MHz}, \mathrm{CDCl}_{3}\right): \delta 2.74\left(\mathrm{~s}, 3 \mathrm{H}, 9-\mathrm{SCH}_{3}\right), 3.03\left(\mathrm{~s}, 3 \mathrm{H}, 2^{\prime}-\mathrm{S}(\mathrm{O}) \mathrm{CH}_{3}\right), 7.56,(\mathrm{dd}, J=$ $4.5,8.2 \mathrm{~Hz}, 1 \mathrm{H}, \mathrm{H}-3), 7.72(\mathrm{~d}, J=5.6 \mathrm{~Hz}, 1 \mathrm{H}, \mathrm{H}-5$ ) $, 7.94(\mathrm{~d}, J=6.5 \mathrm{~Hz}, 1 \mathrm{H}, \mathrm{H}-7), 8.32(\mathrm{~d}, J=$ $6.5 \mathrm{~Hz}, 1 \mathrm{H}, \mathrm{H}-6), 8.61$ (dd, $J=1.5,4.5 \mathrm{~Hz}, 1 \mathrm{H}, \mathrm{H}-2), 8.70$ (dd, $J=1.5,8.2 \mathrm{~Hz}, 1 \mathrm{H}, \mathrm{H}-4) 8.77$ (d, $J=5.6 \mathrm{~Hz}, 1 \mathrm{H}, \mathrm{H}-6)$.

${ }^{13} \mathrm{C}$ NMR $\left(75 \mathrm{MHz}_{3} \mathrm{CDCl}_{3}\right): \delta 15.0\left(9-\mathrm{SCH}_{3}\right), 40.3\left(2^{\prime}-\mathrm{S}(\mathrm{O}) \mathrm{CH}_{3}\right), 100.5(\mathrm{C}-5), 108.8(\mathrm{C}-6)$, $116.9\left(\mathrm{C}^{\prime}\right), 120.6(\mathrm{C}-4 \mathrm{a}), 121.4(\mathrm{C}-3), 128.1$ (C-4), 139.3 (C-5a), 141.6 (C-7), 142.2 (C-2), 143.4 (C-10a), $155.6(\mathrm{C}-9), 157.6\left(\mathrm{C}-6^{\prime}\right), 162.5\left(\mathrm{C}-4^{\prime}\right), 173.6\left(\mathrm{C}-2^{\prime}\right)$.

HRMS (ES): Calcd for $\mathrm{C}_{16} \mathrm{H}_{14} \mathrm{~N}_{5} \mathrm{O}^{32} \mathrm{~S}_{2}\left(\mathrm{MH}^{+}\right) 356.0640$, found 356.0648 .

\section{9-(Methylsulfinyl)-5-[2-(methylsulfinyl)pyrimidin-4-yl]pyrido $\left[3^{\prime}, 2^{\prime}: 4,5\right]$ pyrrolo[1,2-c]} pyrimidine 2.69
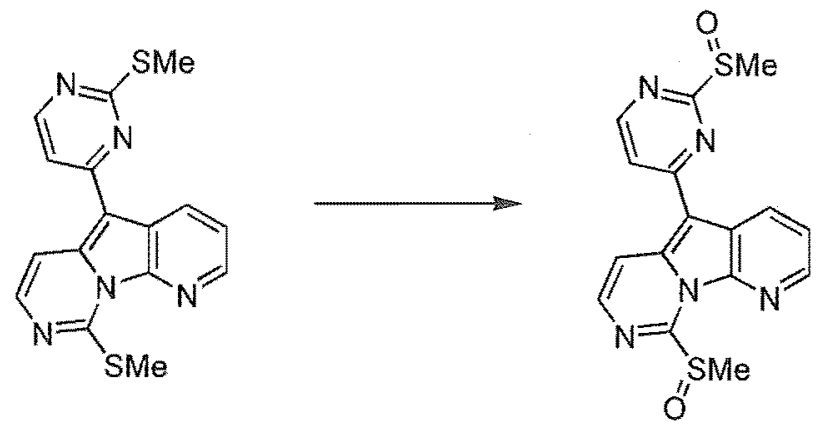

Under atmospheric conditions, a solution of $m$-CPBA in chloroform $(10 \mathrm{mg} / \mathrm{mL})$ was cooled to $-15^{\circ} \mathrm{C}$ (ice/ $\left.\mathrm{NaCl}\right)$, and a portion $(1.9 \mathrm{~mL}, 0.11 \mathrm{mmol})$ was added dropwise to a similarly cooled solution of core structure 2.1 (20 mg, $0.059 \mathrm{mmol})$ in chloroform $(3.4 \mathrm{~mL})$. Analysis by TLC showed all starting material to be consumed. The mixture was allowed to warm to $\sim 0{ }^{\circ} \mathrm{C}$ and was neutralised with sat aq $\mathrm{NaHCO}_{3}$ solution. Extraction with $\mathrm{CH}_{2} \mathrm{Cl}_{2}$ and standard workup gave a yellow solid which was predominantly a mixture of diastereomeric bis-sulfoxides 2.69. The crude mixture was used without purification, however the bis-sulfoxides had the following spectroscopic characteristics: 
${ }^{1} \mathrm{H} \mathrm{NMR}\left(500 \mathrm{MHz}, \mathrm{CDCl}_{3}\right.$ ): (As most signals for the diastereoisomers coincide, they are all quoted as multiplets.) $\delta 3.03(\mathrm{~m}, 3 \mathrm{H}), 3.23(\mathrm{~m}, 3 \mathrm{H}), 7.62-7.65(\mathrm{~m}, 1 \mathrm{H}), 7.77(\mathrm{~m}, 1 \mathrm{H}), 8.23(\mathrm{~m}$, $1 \mathrm{H}), 8.63(\mathrm{~m}, 1 \mathrm{H}), 8.66-8.69(\mathrm{~m}, 1 \mathrm{H}), 8.78-8.80(\mathrm{~m}, 1 \mathrm{H}), 8.85(\mathrm{~m}, 1 \mathrm{H})$.

\section{9-(4-Methoxybenzylamino)-5-[2-(4-methoxybenzylamino)pyrimidin-4-yl]pyrido[3',2':4,5]} pyrrolo[1,2-c] pyrimidine 2.70
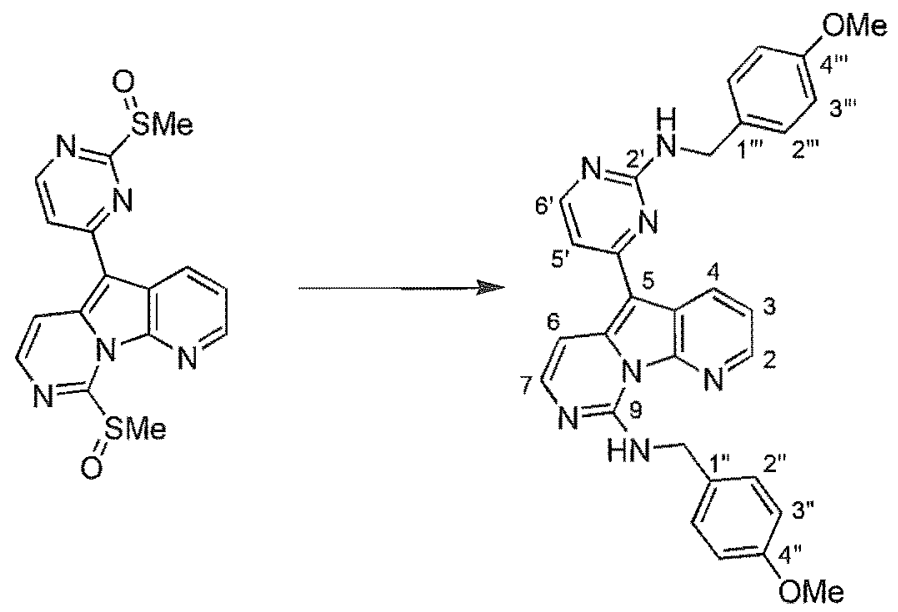

The crude oxidised material 2.69 was heated with an excess of $p$-methoxybenzylamine $(0.20 \mathrm{~mL}$, $1.5 \mathrm{mmol}$ ) at $90^{\circ} \mathrm{C}$ for $14 \mathrm{~h}$. The red paste thus obtained was directly purified by flash chromatography on silica, eluting with $10 \% \mathrm{CH}_{3} \mathrm{CN} / \mathrm{CH}_{2} \mathrm{Cl}_{2}$ then $2-2.5 \% \mathrm{i}-\mathrm{PrOH} / \mathrm{CH}_{2} \mathrm{Cl}_{2}$, to give bis-amine 2.70 as a yellow-orange solid ( $\sim 95 \%$ pure by ${ }^{\mathrm{I}} \mathrm{H}$ NMR spectroscopy, $20 \mathrm{mg}, 62 \%$ over two steps).

Mp: $54-60^{\circ} \mathrm{C}$.

${ }^{1} \mathbf{H ~ N M R}\left(300 \mathrm{MHz}, \mathrm{CDCl}_{3}\right): \delta 3.808\left(\mathrm{~s}, 3 \mathrm{H}, \mathrm{OCH}_{3}\right), 3.811(\mathrm{~s}, 3 \mathrm{H}, \mathrm{OCH}), 4.69(\mathrm{~d}, J=5.9 \mathrm{~Hz}$, $2 \mathrm{H}, 2^{\prime}-\mathrm{NHCH}_{2} \mathrm{Ar}$ ), 4.89 (d, $J=5.4 \mathrm{~Hz}, 2 \mathrm{H}, 9-\mathrm{NHCH}_{2} \mathrm{Ar}$ ), $5.52\left(\mathrm{br}, 1 \mathrm{H}, 2^{\prime}-\mathrm{NH}\right), 6.89-6.93(\mathrm{~m}$, 4H, PMB H-3", PMB H-3"'), 6.98 (d, $J=5.4$ Hz, 1H, H-5'), 7.33-7.42 (m, 6H, H-3, H-6, PMB H-2", PMB H-2"'), 7.63 (d, $J=6.8 \mathrm{~Hz}, 1 \mathrm{H}, \mathrm{H}-7$ ), 8.27 (dd, $J=1.0,5.2 \mathrm{~Hz}, 1 \mathrm{H}, \mathrm{H}-2), 8.31$ (d, $J=$ $\left.5.4 \mathrm{~Hz}, 1 \mathrm{H}, \mathrm{H}-6^{\prime}\right), 8.56$ (br m, 1H, H-4), 10.36 (br t, $J=5.4 \mathrm{~Hz}, 1 \mathrm{H}, 9-\mathrm{N} H$ ).

${ }^{13} \mathrm{C}$ NMR $\left(75 \mathrm{MHz}, \mathrm{CDCl}_{3}\right): \delta 44.3\left(9-\mathrm{NHCH}_{2} \mathrm{Ar}\right), 45.1$ (2'- $\left.\mathrm{NHCH}_{2} \mathrm{Ar}\right), 55.3$ (both $\mathrm{OCH}_{3}$ ), 100.6 (C-5), 101.5 (C-6), 107.9 (C-5'), 113.9 (PMB C-3" or PMB C-3"'), 114.0 (PMB C-3"' or PMB C-3"), 120.0 (C-3), 121.9 (C-4a), 128.4 (C-4), 128.6 (PMB C-2"'), 128.8 (PMB C-2"), 130.3 (PMB C-1"), 131.4 (PMB C-1"'), 138.4 (C-5a), 139.5 (C-2), 143.0 (C-7), 143.4 (C-10a), 
149.0 (C-9), 157.4 (C-6'), 158.7 (PMB C-4" or PMB C-4"'), 158.9 (PMB C-4'" or PMB C-4"), $162.1\left(\mathrm{C}-4^{\prime}\right.$ or $\left.\mathrm{C}-2^{\prime}\right), 162.2\left(\mathrm{C}-2^{\prime}\right.$ or $\left.\mathrm{C}-4^{\prime}\right)$.

IR (KBr): $v_{\max } 3240,2959-2835$ (series of weak bands), 1560, 1512.

MS (EI): $m / z$ (rel intensity) 517 (56), 397 (12), 382 (12), 121 (100).

HRMS (EI): Calcd for $\mathrm{C}_{30} \mathrm{H}_{27} \mathrm{~N}_{7} \mathrm{O}_{2}\left(\mathrm{M}^{+}\right)$517.2226, found 517.2242.

\section{Deoxyvariolin $\mathbb{B} 1.50$}

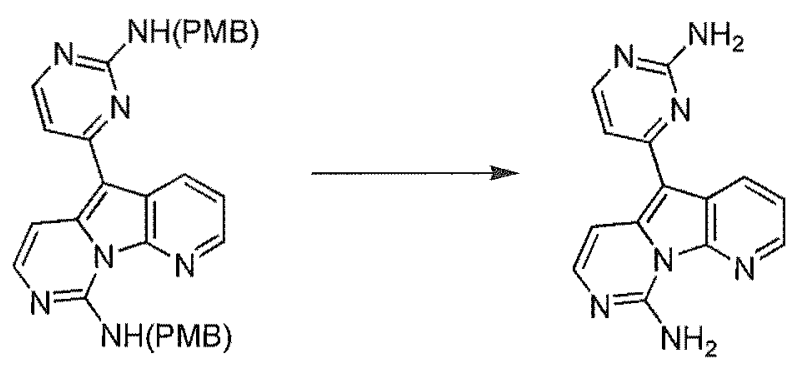

Bis-amine $2.70(95 \%, 6.7 \mathrm{mg}, 0.012 \mathrm{mmol})$ was dissolved in neat $\mathrm{TfOH}(0.05 \mathrm{~mL})$ in a small vial under atmospheric conditions. The vial was capped and the deep red solution was left at $\mathrm{rt}$ for $38 \mathrm{~h}$. The reaction was cooled to $-20^{\circ} \mathrm{C}$ and carefully quenched with cold, conc aq $\mathrm{NH}_{3}$ $\left(\sim 25 \% \mathrm{NH}_{3}, 0.1 \mathrm{~mL}\right)$. The suspension was diluted with water $(1 \mathrm{~mL})$ and mixed thoroughly to ensure complete neutralisation, followed by removal of water in vacuo. The yellow solid was taken up in $\mathrm{CH}_{2} \mathrm{Cl}_{2} / \mathrm{MeOH}(50: 1, \sim 50 \mathrm{~mL})$, filtered through a fine frit to remove ammonium triflate, and dried $\left(\mathrm{Na}_{2} \mathrm{SO}_{4}\right)$ to give the crude product. Flash chromatography on silica, eluting with $8 \% \mathrm{MeOH} / \mathrm{CH}_{2} \mathrm{Cl}_{2}$ then $8 \% \mathrm{MeOH} / \mathrm{CH}_{2} \mathrm{Cl}_{2}+1 \% \mathrm{NEt}_{3}$, followed by flash chromatography on reversed phase silica, eluting with $45 \% \mathrm{MeOH} /$ water then $70 \% \mathrm{MeOH} /$ water $+0.1 \% \mathrm{TFA}$, gave an acidic product. This was dissolved in $\mathrm{CH}_{2} \mathrm{Cl}_{2} / \mathrm{MeOH}$ (20:1) and neutralised with solid $\mathrm{K}_{2} \mathrm{CO}_{3}$. After filtration and concentration, the resulting yellow-brown solid was extracted with boiling $\mathrm{CH}_{2} \mathrm{Cl}_{2}$. The fluorescent yellow solution was filtered and concentrated to give deoxyvariolin $\mathrm{B}(\mathbf{1 . 5 0})$ as a yellow solid (2.0 $\mathrm{mg}, 56 \%)$.

Mp: dec at $\sim 220-240{ }^{\circ} \mathrm{C}\left(\mathrm{lit}^{38} 161-162^{\circ} \mathrm{C}\right)$.

${ }^{1} \mathrm{H}$ NMR $\left(500 \mathrm{MHz},\left(\mathrm{CD}_{3}\right)_{2} \mathrm{SO}\right): \delta 6.51\left(\mathrm{~s}, 2 \mathrm{H}, 2^{\prime}-\mathrm{NH} H_{2}\right), 7.05\left(\mathrm{~d}, J=5.4 \mathrm{~Hz}, 1 \mathrm{H}, \mathrm{H}-5{ }^{\prime}\right), 7.57(\mathrm{dd}$, $J=4.8,8.2 \mathrm{~Hz}, 1 \mathrm{H}, \mathrm{H}-3), 7.62(\mathrm{~d}, J=6.3 \mathrm{~Hz}, 1 \mathrm{H}, \mathrm{H}-7), 7.67$ (d, $J=6.3 \mathrm{~Hz}, 1 \mathrm{H}, \mathrm{H}-6), 8.22$ (d, $J$ 
$\left.=5.4 \mathrm{~Hz}, 1 \mathrm{H}, \mathrm{H}-6^{\prime}\right), 8.44(\mathrm{dd}, J=1.5,4.8 \mathrm{~Hz}, 1 \mathrm{H}, \mathrm{H}-2), 8.5$ (br, $\left.1 \mathrm{H}, 9-\mathrm{NH}\right), 8.90$ (dd, $J=1.5$, $8.2 \mathrm{~Hz}, 1 \mathrm{H}, \mathrm{H}-4), 9.4(\mathrm{br}, 1 \mathrm{H}, 9-\mathrm{NH})$.

${ }^{13} \mathrm{C} \mathrm{NMR}\left(75 \mathrm{MHz},\left(\mathrm{CD}_{3}\right)_{2} \mathrm{SO}\right): \delta 99.6(\mathrm{C}-5), 101.9(\mathrm{C}-6), 107.1(\mathrm{C}-5), 121.0(\mathrm{C}-3), 121.7(\mathrm{C}-$ 4a), 129.4 (C-4), 138.3 (C-5a), 140.3 (C-2), 143.0 (C-10a), 144.0 (C-7), 149.9 (C-9), 158.2 (C6'), $161.6\left(\mathrm{C}-4^{\prime}\right), 163.6\left(\mathrm{C}-2^{\prime}\right)$.

IR (KBr): $\mathrm{U}_{\max } 3304,3142-2849$ (series of weak bands), 1647, 1574, 1514, 1472, 1456, 1269. MS (EI): $m / z$ (rel intensity) 277 (100), $236(54)$.

HRMS (EI): Calcd for $\mathrm{C}_{14} \mathrm{H}_{11} \mathrm{~N}_{7}\left(\mathrm{M}^{+}\right) 277.1076$, found 277.1072. 


\subsection{Experiments Described im Chapter Three}

Ethyll 2-cyano-3-methoxybut-2-enoate $3.4^{96}$

$$
\mathrm{EtO}_{2} \mathrm{C} \frown \mathrm{CN}+(\mathrm{MeO})_{3} \mathrm{CMe}
$$<smiles>CCOC(C#N)=C(C)OC</smiles>

This reaction was performed in a two-necked flask with a $15 \mathrm{~cm}$ Vigreux column and condenser fitted to one neck. A graduated receiver was attached to the condenser so that the volume of distillate could be ascertained. Trimethylorthoacetate $(61.4 \mathrm{~g}, 0.511 \mathrm{~mol})$, ethyl cyanoacetate $(47.2 \mathrm{~g}, 0.417 \mathrm{~mol})$ and acetic acid $(2.4 \mathrm{~mL})$ were heated together in an oil bath $\left(\sim 120^{\circ} \mathrm{C}\right)$, causing $\mathrm{MeOH}$ to distil. Further portions of acetic acid $(1.2 \mathrm{~mL})$ were added after 19,29 and 38 $\mathrm{mL}$ of $\mathrm{MeOH}$ had collected. The solid mass that remained was recrystallised from hot benzene to give the title compound 3.4 as fine off-white needles $(49.3 \mathrm{~g}, 70 \%)$. ${ }^{1} \mathrm{H}$ NMR analysis indicated the presence of only one stereoisomer, however the actual stereochemistry $(E$ or $Z$ ) is undefined.

Mp: $130-132{ }^{\circ} \mathrm{C}\left(1 \mathrm{lit}^{96} 133^{\circ} \mathrm{C}\right)$.

${ }^{1} \mathrm{H}$ NMR $\left(500 \mathrm{MHz}, \mathrm{CDCl}_{3}\right): \delta 1.32\left(\mathrm{t}, J=7.1 \mathrm{~Hz}, 3 \mathrm{H}, \mathrm{OCH}_{2} \mathrm{CH}_{3}\right), 2.62(\mathrm{~s}, 3 \mathrm{H}$, $\left.\mathrm{CH}_{3} \mathrm{C}(\mathrm{OMe})=\mathrm{CR}_{2}\right), 3.99\left(\mathrm{~s}, 3 \mathrm{H}, \mathrm{OCH}_{3}\right), 4.23\left(\mathrm{q}, J=7.1 \mathrm{~Hz}, 2 \mathrm{H}, \mathrm{OCH}_{2} \mathrm{CH}_{3}\right)$.

${ }^{13} \mathrm{C}$ NMR $\left(75 \mathrm{MHz}, \mathrm{CDCl}_{3}\right): \delta 14.0\left(\mathrm{OCH}_{2} \mathrm{CH}_{3}\right), 14.9(\mathrm{C}-4), 56.6\left(\mathrm{OCH}_{3}\right), 61.0\left(\mathrm{OCH}_{2} \mathrm{CH}_{3}\right)$, $85.6(\mathrm{C}-2), 114.9(\mathrm{CN}), 163.6(\mathrm{C}-1), 184.4,(\mathrm{C}-3)$.

IR (KBr): $v_{\max } 2216,1713,1582,1319,1281,1144,1088$.

MS (EI): $m / z$ (rel intensity) 169 (36), $124(100), 123$ (37).

HRMS (EI): Calcd for $\mathrm{C}_{18} \mathrm{H}_{11} \mathrm{NO}_{3}\left(\mathrm{M}^{+}\right)$169.0739, found 169.0743 . 
Ethyl 2-cyano-5-(dimethylamino)-3-methoxypenta-2,4-dienoate $3.5^{96}$<smiles>CCOC(=O)/C(C#N)=C(/N)OC</smiles>

$\mathrm{Me}_{2} \mathrm{NCH}(\mathrm{OMe})_{2}$<smiles>CCOC(=O)/C(C#N)=C(/C=C/NC)OC</smiles>

Enamine 3.5 was prepared from $3.4(57 \mathrm{~g})$ using the procedure of Kasum and Prager, ${ }^{96}$ and was obtained as a bright yellow solid ( $\sim 92 \%$ pure by ${ }^{1} \mathrm{H}$ NMR spectroscopy, $\left.65 \mathrm{~g}, 79 \%\right)$. The impurities were conveniently removed later in the synthesis. A small sample, however, was recrystallised from EtOAc/hexanes for analytical purposes. ${ }^{1} \mathrm{H}$ NMR analysis indicated the presence of only one stereoisomer, however the actual stereochemistry $(E$ or $Z)$ is undefined.

Mp: $68-72{ }^{\circ} \mathrm{C}\left(\mathrm{lit}^{96} 68.5-70^{\circ} \mathrm{C}\right)$.

${ }^{1} \mathrm{H} \mathbb{N M R}\left(500 \mathrm{MHz}, \mathrm{CDCl}_{3}\right): \delta 1.31\left(\mathrm{t}, J=7.1 \mathrm{~Hz}, 3 \mathrm{H}, \mathrm{OCH}_{2} \mathrm{CH}_{3}\right), 2.96\left(\mathrm{~s}, 3 \mathrm{H}, \mathrm{NCH}_{3}\right), 3.18(\mathrm{~s}$, $\left.3 \mathrm{H}, \mathrm{NCH}_{3}\right), 4.02\left(\mathrm{~s}, 3 \mathrm{H}, \mathrm{OCH}_{3}\right), 4.20\left(\mathrm{q}, J=7.1 \mathrm{~Hz}, 2 \mathrm{H}, \mathrm{OCH}_{2} \mathrm{CH}_{3}\right), 6.21$ (br, H-4), 7.48 (d, $J=$ 12.7 Hz, H-5).

${ }^{13} \mathrm{C}$ NMR $\left(75 \mathrm{MHz}, \mathrm{CDCl}_{3}\right): \delta 14.4\left(\mathrm{OCH}_{2} \mathrm{CH}_{3}\right), 37.5\left(\mathrm{NCH}_{3}\right), 45.6\left(\mathrm{NCH}_{3}\right), 60.0\left(\mathrm{OCH}_{2} \mathrm{CH}_{3}\right)$, $62.4\left(\mathrm{OCH}_{3}\right), 75.7(\mathrm{C}-2), 91.5(\mathrm{C}-4), 119.1(\mathrm{CN}), 152.9(\mathrm{C}-5), 166.0$ (br, C-1), 181.6, (C-3).

$\mathbb{I R}(\mathrm{KBr}): \mathrm{v}_{\max } 2197,1688,1622,1529,1391,1232,1060$.

MS (EI): $m / z$ (rel intensity) 224 (100), 179 (73), 152 (28), 151 (39), 121 (23), 119 (50), 112 (61) 108 (44), 98 (39), 82 (38).

HRMS (EI): Calcd for $\mathrm{C}_{11} \mathrm{H}_{16} \mathrm{~N}_{2} \mathrm{O}_{3}\left(\mathrm{M}^{+}\right)$224.1161, found 224.1153.

\section{Ethyl 2-chloro-4-methoxymicotinoate 3.7}

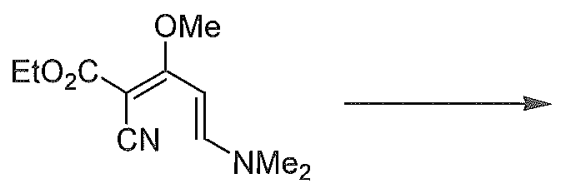<smiles>CCOC(=O)c1c(OC)ccnc1Cl</smiles>

Enamine $3.5(2.30 \mathrm{~g}, 10.3 \mathrm{mmol})$ was stirred with freshly distilled acetic acid $(10 \mathrm{~mL})$ at $67^{\circ} \mathrm{C}$ for $5.5 \mathrm{~h}$, while dry $\mathrm{HCl}$ gas (prepared by the action of conc $\mathrm{H}_{2} \mathrm{SO}_{4}$ on solid $\mathrm{NH}_{4} \mathrm{Cl}$ and a few drops of conc hydrochloric acid, and dried by bubbling through conc $\mathrm{H}_{2} \mathrm{SO}_{4}$ ) was constantly bubbled into the reaction. After cooling, the reaction mixture was poured into water $(20 \mathrm{ml})$ and 
neutralised with solid $\mathrm{NaHCO}_{3}$ to $\mathrm{pH}$ 8. Standard workup $\left(\mathrm{CH}_{2} \mathrm{Cl}_{2}\right)$ gave the crude material which was partially purified by Kugelrohr distillation $\left(175^{\circ} \mathrm{C}\right.$ at $0.07 \mathrm{~mm} \mathrm{Hg}$, after removing more volatile components) to give a yellow oil. The remaining impurities were removed in the following step, however a small sample of the ester 3.7 was purified by flash chromatography on silica, eluting with 50\% EtOAc/hexanes, to give a colourless oil.

${ }^{1} \mathrm{H}$ NMR $\left(500 \mathrm{MHz}, \mathrm{CDCl}_{3}\right): \delta 1.38\left(\mathrm{t}, J=7.2 \mathrm{~Hz}, 3 \mathrm{H}, \mathrm{OCH}_{2} \mathrm{CH}_{3}\right), 3.89\left(\mathrm{~s}, 3 \mathrm{H}, \mathrm{OCH}_{3}\right), 4.41(\mathrm{q}$, $\left.J=7.2 \mathrm{~Hz}, 2 \mathrm{H}, \mathrm{OCH}_{2} \mathrm{CH}_{3}\right), 6.81(\mathrm{~d}, J=5.9 \mathrm{~Hz}, 1 \mathrm{H}, \mathrm{H}-5), 8.28(\mathrm{~d}, J=5.9 \mathrm{~Hz}, 1 \mathrm{H}, \mathrm{H}-6)$.

${ }^{13} \mathrm{C}$ NMR $\left(126 \mathrm{MHz}, \mathrm{CDCl}_{3}\right): \delta 13.7\left(\mathrm{OCH}_{2} \mathrm{CH}_{3}\right), 56.2\left(\mathrm{OCH}_{3}\right), 61.9\left(\mathrm{OCH}_{2} \mathrm{CH}_{3}\right), 106.0(\mathrm{C}-5)$, 119.5 (C-3), 147.7 (C-2), 150.9 (C-6), 163.7 ( $C=\mathrm{O}), 163.9$ (C-4).

$\mathbb{I R}(\mathrm{KBr}): v_{\max } 1738,1580,1477,1304,1040$.

MS (EI): $m / z$ (rel intensity) 215/217 (12/6), $187 / 189$ (15/5), 170/172 (100/32), 112/114 (15/5).

HRMS (EI): Calcd for $\mathrm{C}_{9} \mathrm{H}_{10}{ }^{35} \mathrm{ClNO}_{3}\left(\mathrm{M}^{+}\right)$215.0349, found 215.0347 .

\section{2-Chloro-4-methoxynicotinic acid 3.9}<smiles>CCOC(=O)c1c(OC)ccnc1Cl</smiles><smiles>COc1ccnc(Cl)c1C(=O)O</smiles>

The partially purified ester $\mathbf{3 . 7}$ was hydrolysed by refluxing with $1.5 \mathrm{M}$ aq $\mathrm{NaOH}$ solution (65 $\mathrm{mL}$ ) for $0.5 \mathrm{~h}$. After cooling, the alkaline solution was washed with EtOAc $(\times 3)$ and then acidified to $\mathrm{pH} 2$ with conc hydrochloric acid. The acidified solution was saturated with solid $\mathrm{NaCl}$, extracted with THF $(\times 4)$ and dried $\left(\mathrm{Na}_{2} \mathrm{SO}_{4}\right)$ to give carboxylic acid 3.9 as a white solid (1.49 g, 77\% over two steps).

Mp: $170-176^{\circ} \mathrm{C}(\mathrm{dec})$.

${ }^{1} \mathrm{H}$ NMR $\left(500 \mathrm{MHz},\left(\mathrm{CD}_{3}\right)_{2} \mathrm{SO}\right): \delta 3.91\left(\mathrm{~s}, 3 \mathrm{H}, \mathrm{OCH}_{3}\right), 7.23(\mathrm{~d}, J=5.9 \mathrm{~Hz}, 1 \mathrm{H}, \mathrm{H}-5), 8.34(\mathrm{~d}, J$ $=5.9 \mathrm{~Hz}, 1 \mathrm{H}, \mathrm{H}-6)$.

${ }^{13} \mathrm{C} \mathrm{NMR}\left(75 \mathrm{MHz}, \mathrm{CDCl}_{3}\right): \delta 57.0\left(\mathrm{OCH}_{3}\right), 107.8(\mathrm{C}-5), 120.7(\mathrm{C}-3), 146.1(\mathrm{C}-2), 151.3(\mathrm{C}-6)$, $163.6(\mathrm{C}-4), 165.0(C=\mathrm{O})$.

$\mathbb{I R}(\mathrm{KBr})$ : $v_{\max } 3107,2970-2400$ (series of broad bands), 1719, 1585, 1304, 1042. 
MS (EI): $m / z$ (rel intensity) $187 / 189$ (83/28), 170/172 (86/30), 140 (53), 133 (45), 123 (63), $112 / 114(62 / 22), 105(100), 78(33), 77(32)$.

HRMS (ED): Calcd for $\mathrm{C}_{7} \mathrm{H}_{6}{ }^{35} \mathrm{CINO}_{3}\left(\mathrm{M}^{+}\right) 187.0036$, found 187.0037 .

\section{2-Chloro-4-methoxynicotinoyl chloride 1.67}<smiles>COc1ccnc(Cl)c1C(=O)Cl</smiles>

Carboxylic acid $3.9(1.27 \mathrm{~g}, 6.77 \mathrm{mmol})$, oxalyl chloride $(2.4 \mathrm{~mL}, 28 \mathrm{mmol})$ and DMF (3 drops) were stirred in $\mathrm{CH}_{2} \mathrm{Cl}_{2}$ at $\mathrm{rt}$ for $19 \mathrm{~h}$, with a $\mathrm{CaCl}_{2}$ drying tube used to exclude atmospheric moisture. After concentration in vacuo, the crude product was purified by Kugelrohr distillation $\left(150{ }^{\circ} \mathrm{C}, 0.07 \mathrm{~mm} \mathrm{Hg}\right)$ to give acid chloride 1.67 as a white crystalline solid $(1.36 \mathrm{~g}, 97 \%)$.

Mp: $66-69^{\circ} \mathrm{C}$, sweats at $62^{\circ} \mathrm{C}$.

${ }^{1} \mathrm{H}$ NMR $\left(500 \mathrm{MHz} \mathrm{CDCl}_{3}\right): \delta 3.98\left(\mathrm{~s}, 3 \mathrm{H}, \mathrm{OCH}_{3}\right), 6.89(\mathrm{~d}, J=5.9 \mathrm{~Hz}, 1 \mathrm{H}, \mathrm{H}-5), 8.36(\mathrm{~d}, J=$ $5.9 \mathrm{~Hz}, 1 \mathrm{H}, \mathrm{H}-6)$.

${ }^{13} \mathrm{C} \mathrm{NMR}\left(126 \mathrm{MHz}, \mathrm{CDCl}_{3}\right) ; \delta 56.8\left(\mathrm{OCH}_{3}\right), 106.4(\mathrm{C}-5), 123.9(\mathrm{C}-3), 146.1(\mathrm{C}-2), 152.1(\mathrm{C}-$ 6), $162.9(\mathrm{C}-4), 164.3(\mathrm{C}=\mathrm{O})$.

$\operatorname{IR}\left(\mathrm{CDCl}_{3}\right): v_{\max } 1792,1578,1302,1047$.

MS (EI): $m / z$ (rel intensity) 170/172 (100/34), 112/114 (27/9).

IIRMS (ED): Calcd for $\mathrm{C}_{7} \mathrm{H}_{5}{ }^{35} \mathrm{CINO}_{2}\left(\mathrm{M}^{+}-\mathrm{Cl}\right) 170.0009$, found 170.0007 . 
<smiles>COc1ccnc(Cl)c1C(=O)Cl</smiles><smiles>COc1nccc(C(O)(c2ccnc(S(C)(=O)=O)n2)c2c(OC)ccnc2Cl)n1</smiles>

The reactor (Figure 2.8, p 41) was charged with iodide 2.12 (4.44 g, 17.6 mmol) and freshly distilled THF $(180 \mathrm{~mL})$, and cooled to $-95^{\circ} \mathrm{C}\left(\mathrm{MeOH} / \mathrm{N}_{2}\right)$. A solution of $n$-BuLi in hexanes (1.55 M, $11.4 \mathrm{~mL}, 17.7 \mathrm{mmol}$ ) was added dropwise to the stirred solution over $19 \mathrm{~min}$, while maintaining the temperature at $-95^{\circ} \mathrm{C}$. After $30 \mathrm{~min}$, a solution of acid chloride $1.67(1.21 \mathrm{~g}$, $5.86 \mathrm{mmol})$ in THF $(9 \mathrm{~mL})$ was added to the dark mixture over $7 \mathrm{~min}$. The reaction was stirred for $4.5 \mathrm{~h}$ at $-95^{\circ} \mathrm{C}$, quenched with $\mathrm{MeOD}(1.2 \mathrm{~mL})$ and allowed to warm to rt. Excess THF was removed in vacuo and the residue was taken up in water/EtOAc and extracted with EtOAc ( $\times 4)$. The extracts were washed with brine and dried over $\mathrm{MgSO}_{4}$. Partial concentration of the EtOAc solution gave a precipitate of the pyrimidine trimer 2.22 , which was filtered and washed with a little more EtOAc. Concentration of the filtrate and washings gave the crude material. Flash chromatography on silica, eluting with 55-80\% EtOAc/hexanes, gave triarylmethanol $\mathbf{1 . 6 5}$ as a tan solid ( $\sim 93 \%$ pure by ${ }^{1} \mathrm{H}$ NMR spectroscopy, $\left.383 \mathrm{mg}, 14 \%\right)$.

The spectroscopic data for $\mathbf{1 . 6 5}$ is listed on p 198. Other (new) compounds isolated from this reaction and their spectroscopic data are listed below:

a) (2-Chloro-4-methoxypyridin-3-yl)-[2-(methylsulfanyl)pyrimidin-4-yl]methanome 3.10<smiles>COc1ccnc(Cl)c1C(=O)c1ccnc(S(C)(=O)=O)n1</smiles>

This compound was isolated as colourless blocks after recrystallisation from EtOAc. 
Mp: $148-151^{\circ} \mathrm{C}(\mathrm{EtOAc})$

${ }^{1} \mathrm{H}$ NMR $\left(500 \mathrm{MHz}_{\mathrm{H}} \mathrm{CDCl}_{3}\right): \delta 2.31(\mathrm{~s}, 3 \mathrm{H}, \mathrm{SCH}), 3.81\left(\mathrm{~s}, 3 \mathrm{H}, \mathrm{OCH}_{3}\right), 6.89(\mathrm{~d}, J=5.9 \mathrm{~Hz}, 1 \mathrm{H}$, $\mathrm{H}-5), 7.64\left(\mathrm{~d}, J=4.9 \mathrm{~Hz}, 1 \mathrm{H}, \mathrm{H}-5^{\prime}\right), 8.38(\mathrm{~d}, J=5.9 \mathrm{~Hz}, 1 \mathrm{H}, \mathrm{H}-6), 8.80\left(\mathrm{~d}, J=4.9 \mathrm{~Hz}, 1 \mathrm{H}, \mathrm{H}-6^{\prime}\right)$. ${ }^{13} \mathrm{C}$ NMR $\left(75 \mathrm{MHz}, \mathrm{CDCl}_{3}\right): \delta 14.0\left(\mathrm{SCH}_{3}\right), 56.3\left(\mathrm{OCH}_{3}\right), 105.9(\mathrm{C}-5), 112.7\left(\mathrm{C}-5^{\prime}\right), 122.9(\mathrm{C}-$ 3), $148.2(\mathrm{C}-2), 151.5(\mathrm{C}-6), 158.1\left(\mathrm{C}-4^{\prime}\right), 159.3\left(\mathrm{C}^{\prime} 6^{\prime}\right), 165.0(\mathrm{C}-4), 173.7\left(\mathrm{C}-2^{\prime}\right), 191.9(\mathrm{C}=\mathrm{O})$.

$\operatorname{IR}\left(\mathrm{CDCl}_{3}\right): v_{\max } 1705,1578,1555,1047$.

MS (EI): $m / z$ (rel intensity) 295/297 (7/3), 260 (97), 170/172 (100/46), 112 (40).

HRMS (EI): Calcd for $\mathrm{C}_{12} \mathrm{H}_{10}{ }^{35} \mathrm{ClN}_{3} \mathrm{O}_{2}{ }^{32} \mathrm{~S}\left(\mathrm{M}^{+}\right)$295.0182, found 295.0178 .

Crystal data and structure refinement for $\mathbf{3 . 1 0}$

Empirical formula

Formula weight

Temperature

Wavelength

Crystal system

Space group

Unit cell dimensions

Volume

Z

Density (calculated)

Absorption coefficient

$\mathrm{F}(000)$

Crystal size

Theta range for data collection

Index ranges

Reflections collected

Independent reflections

Completeness to theta $=26.52^{\circ}$

Absorption correction

Refinement method

Data / restraints / parameters

Goodness-of-fit on $\mathrm{F}^{2}$

Final $R$ indices $[\mathrm{I}>2$ sigma(I)]
$\mathrm{C}_{12} \mathrm{H}_{10} \mathrm{ClN}_{3} \mathrm{O}_{2} \mathrm{~S}$

295.74

$168(2) \mathrm{K}$

$0.71073 \AA$

Monoclinic

$\mathrm{P} 2(1) / \mathrm{c}$

$\mathrm{a}=7.486(3) \AA$

$\alpha=90^{\circ}$.

$\mathrm{b}=22.718(10) \AA$

$\beta=93.297(7)^{\circ}$.

$\mathrm{c}=7.591$

(3) $\AA$

$1288.8(10) \AA^{3}$

4

$1.524 \mathrm{Mg} / \mathrm{m}^{3}$

$0.459 \mathrm{~mm}^{-1}$

608

$0.5 \times 0.4 \times 0.22 \mathrm{~mm}^{3}$

2.73 to $26.52^{\circ}$.

$-9 \leq \mathrm{h} \leq 9,-28 \leq \mathrm{k} \leq 28,-3 \leq 1 \leq 7$

5356

$2356[\mathrm{R}(\mathrm{int})=0.0847]$

$87.9 \%$

Empirical

Full-matrix least-squares on $\mathrm{F}^{2}$

$2356 / 0 / 174$

1.114

$\mathrm{R} 1=0.0902, \mathrm{wR} 2=0.2170$ 
$\mathrm{R}$ indices (all data)

$\mathrm{R} 1=0.1311, \mathrm{wR} 2=0.2488$

Largest diff. peak and hole

0.521 and -0.744 e. $\AA^{-3}$

b) (2-Chloro-4-methoxypyridin-3-yl)-[2-(methylsulfanyl)pyrimidim-4-yl] methanol 3.11

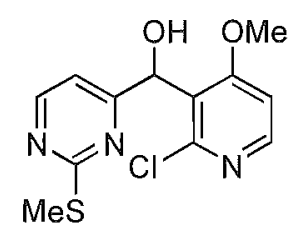

This compound was isolated as a viscous gum.

${ }^{1} \mathrm{H}$ NMR $\left(500 \mathrm{MHz}, \mathrm{CDCl}_{3}\right): \delta 2.51\left(\mathrm{~s}, 3 \mathrm{H}, \mathrm{SCH} H_{3}\right), 3.78\left(\mathrm{~s}, 3 \mathrm{H}, \mathrm{OCH}_{3}\right), 4.62(\mathrm{~d}, J=7.3 \mathrm{~Hz}, 1 \mathrm{H}$, $\mathrm{OH}), 6.30\left(\mathrm{~d}, J=7.3 \mathrm{~Hz}, 1 \mathrm{H}, \mathrm{Ar}_{2} \mathrm{CHOH}\right), 6.78(\mathrm{~d}, J=5.4 \mathrm{~Hz}, 1 \mathrm{H}, \mathrm{H}-5), 6.93(\mathrm{~d}, J=4.9 \mathrm{~Hz}, 1 \mathrm{H}$, H-5'), 8.26 (d, $J=5.4 \mathrm{~Hz}, 1 \mathrm{H}, \mathrm{H}-6), 8.43$ (d, $\left.J=4.9 \mathrm{~Hz}, 1 \mathrm{H}, \mathrm{H}-6^{\prime}\right)$.

${ }^{13} \mathrm{C}$ NMR $\left(75 \mathrm{MHz}, \mathrm{CDCl}_{3}\right): \delta 14.1\left(\mathrm{SCH}_{3}\right), 56.2\left(\mathrm{OCH}_{3}\right), 69.5\left(\mathrm{Ar}_{2} \mathrm{CHOH}\right), 106.6(\mathrm{C}-5), 112.2$ (C-5'), 123.4 (C-3), 150.4 (C-6), 151.9 (C-2), 157.1 (C-6'), 165.5 (C-4), 168.9 (C-4'), 171.9 (C$\left.2^{\prime}\right)$.

$\mathbb{I R}\left(\mathrm{CDCl}_{3}\right): \mathrm{v}_{\max } 3450,1578,1562,1043$.

MS (EI): $m / z$ (rel intensity) 297/299 (32/13), 262 (100), 260 (22), 244 (32), 172/174 (80/25), 170 (32), $136(33), 125$ (34), 111 (38), 78 (49).

HRMS (EI): Calcd for $\mathrm{C}_{12} \mathrm{H}_{12}{ }^{35} \mathrm{ClN}_{3} \mathrm{O}_{2}{ }^{32} \mathrm{~S}\left(\mathrm{M}^{+}\right)$297.0339, found 297.0342.

c) Methyl 2-chloro-4-methoxynicotinoate 3.12<smiles>COc1ccnc(Cl)c1C(C)=O</smiles>

This compound was isolated as a colourless oil.

${ }^{1} \mathrm{HI} \mathrm{NMR}\left(500 \mathrm{MHz}, \mathrm{CDCl}_{3}\right): \delta 3.90\left(\mathrm{~s}, 3 \mathrm{H}, 4-\mathrm{OCH}_{3}\right), 3.94\left(\mathrm{~s}, 3 \mathrm{H}, \mathrm{CO}_{2} \mathrm{CH}_{3}\right), 6.83(\mathrm{~d}, J=5.9 \mathrm{~Hz}$, $1 \mathrm{H}, \mathrm{H}-5), 8.30(\mathrm{~d}, J=5.9 \mathrm{~Hz}, 1 \mathrm{H}, \mathrm{H}-6)$. 
${ }^{13} \mathrm{C} \mathbb{N M R}\left(126 \mathrm{MHz}, \mathrm{CDCl}_{3}\right): \delta 52.8\left(\mathrm{CO}_{2} \mathrm{CH}_{3}\right), 56.3\left(4-\mathrm{OCH}_{3}\right), 106.0(\mathrm{C}-5), 119.2(\mathrm{C}-3), 147.9$ (C-2), $151.1(\mathrm{C}-6), 164.0(\mathrm{C}-4), 164.3(C=\mathrm{O})$.

$\mathbb{I R}\left(\mathrm{CDCl}_{3}\right): \mathrm{v}_{\max } 1738,1582,1306,1047$.

MS (EI): $m / z$ (rel intensity) 201/203 (21/7), 170/172 (100/38), 112/114 (14/5).

HRMS (EI): Calcd for $\mathrm{C}_{8} \mathrm{H}_{8}{ }^{35} \mathrm{ClNO}_{3}\left(\mathrm{M}^{+}\right)$201.0193, found 201.0183.

\section{4-Methoxypyridine- $N$-oxide $3.18^{108}$}

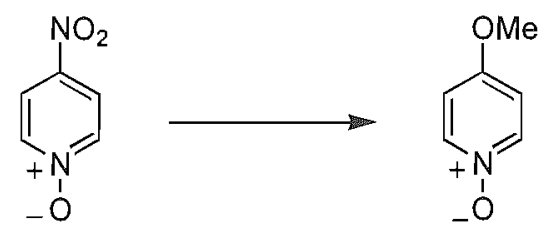

A solution of sodium methoxide, prepared by dissolving sodium $(2.00 \mathrm{~g}, 87.0 \mathrm{mmol})$ in dry $\mathrm{MeOH}(100 \mathrm{~mL})$, was quickly poured into a stirred suspension of 4-nitropyridine- $N$-oxide (12.0 g, $85.7 \mathrm{mmol})$ in $\mathrm{MeOH}(80 \mathrm{~mL})$. Dissolution was complete within $1 \mathrm{~h}$ and the reaction was left stirring in a stoppered flask overnight. Partial concentration of the yellow solution produced a precipitate, which was filtered and washed with $\mathrm{MeOH}(2 \times 10 \mathrm{~mL})$. The filtrate and washings were evaporated to dryness, and the solid that remained was extracted with boiling $\mathrm{CH}_{2} \mathrm{Cl}_{2}$ to give (after filtration and concentration) a yellow-brown solid. Recrystallisation from acetone gave fine off-white needles of 3.18, which were dried overnight in a vacuum dessicator at 0.07 $\mathrm{mm} \mathrm{Hg},(8.04 \mathrm{~g}, 75 \%)$.

Mp: $103-106^{\circ} \mathrm{C}\left(1 \mathrm{lit}^{128} 82-85^{\circ} \mathrm{C}\right.$ for the hydrate).

${ }^{1}{ }_{\mathrm{H}} \mathrm{NMR}\left(500 \mathrm{MHz}, \mathrm{CDCl}_{3}\right): \delta 3.86(\mathrm{~s}, 3 \mathrm{H}), 6.80(\mathrm{~d}, J=7.6 \mathrm{~Hz}, 2 \mathrm{H}), 8.12(\mathrm{~d}, J=7.6 \mathrm{~Hz}, 2 \mathrm{H})$. 
4-Methoxy-2(11H)-pyridone $3.19^{110}$

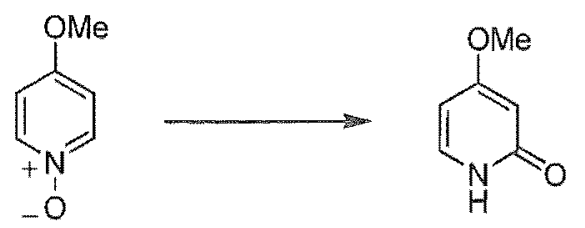

This was prepared from 4-methoxypyridine- $N$-oxide $(3.18,5.00 \mathrm{~g})$ following a literature procedure. ${ }^{110}$ Recrystallisation from $\mathrm{CH}_{3} \mathrm{CN}$ gave colourless plates (3.05 g, 61\%).

Mp: not well defined $\left(\sim 150-170^{\circ} \mathrm{C}\right)$. No melting point was given in reference 110 .

${ }^{1} \mathbf{H}$ NMR $\left(500 \mathrm{MHz}, \mathrm{CDCl}_{3}\right): \delta 3.79(\mathrm{~s}, 3 \mathrm{H}), 5.88(\mathrm{~d}, J=2.5 \mathrm{~Hz}, 1 \mathrm{H}), 5.97(\mathrm{dd}, J=2.5,7.4 \mathrm{~Hz}$, $1 \mathrm{H}), 7.20(\mathrm{~d}, J=7.4 \mathrm{~Hz}, 1 \mathrm{H}), 12.6(\mathrm{br}, 1 \mathrm{H})$.

2-Chloro-4-methoxypyridine $3.8^{103-105}$<smiles>COc1ccnc(Cl)c1</smiles>

4-Methoxy-2(1H)-pyridone (3.19) (2.86 $\mathrm{g}, 22.8 \mathrm{mmol})$ was stirred with $\mathrm{POCl}_{3}(28 \mathrm{~mL})$ at $95^{\circ} \mathrm{C}$ for $16 \mathrm{~h}$. Excess $\mathrm{POCl}_{3}$ was removed in vacuo and the resultant viscous oil was cooled in ice and carefully neutralised with sat aq $\mathrm{NaHCO}_{3}$ solution. The mixture was extracted with EtOAc and worked up in the usual manner to give a golden oil. Flash chromatography on silica, eluting with 30\% EtOAc/hexanes, gave 2-chloro-4-methoxypyridine (3.8) as a colourless oil (2.60 g, $79 \%)$.

${ }^{1} \mathrm{H} \mathrm{NMR}\left(300 \mathrm{MHz}, \mathrm{CDCl}_{3}\right): \delta 3.85\left(\mathrm{~s}, 3 \mathrm{H}, \mathrm{OCH}_{3}\right), 6.75(\mathrm{dd}, J=2.3,5.9 \mathrm{~Hz}, 1 \mathrm{H}, \mathrm{H}-5), 6.83(\mathrm{~d}$, $J=2.3 \mathrm{~Hz}, 1 \mathrm{H}, \mathrm{H}-3), 8.18(\mathrm{~d}, J=5.9 \mathrm{~Hz}, 1 \mathrm{H}, \mathrm{H}-6)$.

${ }^{13} \mathrm{C} \mathrm{NMR}\left(75 \mathrm{MHz}, \mathrm{CDCl}_{3}\right): \delta 55.6\left(\mathrm{OCH}_{3}\right), 109.4(\mathrm{C}-3), 109.7(\mathrm{C}-5), 150.2(\mathrm{C}-6), 152.6(\mathrm{C}-2)$, $167.3(\mathrm{C}-4)$.

$\mathbb{I R}(\mathrm{KBr}): \mathrm{v}_{\max } 1589,1556,1032$.

MS (EI): $m / z$ (rel intensity) 143/145 (100/43), 113/115 (32/10), 108 (39), 78 (19).

HRMS (EI): Calcd for $\mathrm{C}_{6} \mathrm{H}_{6}{ }^{35} \mathrm{CINO}\left(\mathrm{M}^{\dagger}\right) 143.0138$, found 143.0141 . 
Bis-[2-(methylsulfanyl)pyrimidin-4-yll methanone 3.15<smiles>CCOC(=O)OCC</smiles>

The reactor (Figure 2.8, p 41) was charged with iodide $\mathbf{2 . 1 2}$ (3.90 g, $15.5 \mathrm{mmol}$ ) and distilled THF ( $47 \mathrm{~mL})$, and cooled to $-97^{\circ} \mathrm{C}\left(\mathrm{MeOH} / \mathrm{N}_{2}\right)$. A solution of $n$-BuLi in hexanes $(1.55 \mathrm{M}, 10.0$ $\mathrm{mL}, 15.5 \mathrm{mmol}$ ) was slowly added to the stirred solution over $21 \mathrm{~min}$, while maintaining the temperature at $-97^{\circ} \mathrm{C}$. After $30 \mathrm{~min}$, a solution of diethyl carbonate $(0.94 \mathrm{~mL}, 7.8 \mathrm{mmol})$ in THF ( $4 \mathrm{~mL}$ ) was added over about $3 \mathrm{~min}$. After $15 \mathrm{~min}$ at $-97^{\circ} \mathrm{C}$ the reaction was allowed to warm to $-35^{\circ} \mathrm{C}$ over $2 \mathrm{~h}$, and then to rt. The reaction mixture was shaken with sat aq $\mathrm{NH}_{4} \mathrm{Cl}$ solution, extracted with EtOAc and subjected to standard workup. The crude material was partially purified by Kugelrohr distillation $\left(160{ }^{\circ} \mathrm{C}, 0.03 \mathrm{~mm} \mathrm{Hg}\right.$ after removing more volatile components), then subsequently by flash chromatography on silica, eluting with 25, 30 and then $50 \%$ EtOAc/hexanes, to give ketone $\mathbf{3 . 1 5}$ as a pale yellow solid (1.14 g, 53\%).

Mp: $106-107^{\circ} \mathrm{C}$ (benzene/hexanes).

${ }^{1} \mathrm{H}$ NMR $\left(500 \mathrm{MHz}, \mathrm{CDCl}_{3}\right): \delta 2.51\left(\mathrm{~s}, 6 \mathrm{H}, \mathrm{SCH}_{3}\right), 7.54(\mathrm{~d}, J=4.9 \mathrm{~Hz}, 2 \mathrm{H}, \mathrm{H}-5), 8.79(\mathrm{~d}, J=$ $4.9 \mathrm{~Hz}, 2 \mathrm{H}, \mathrm{H}-6)$.

${ }^{13} \mathrm{C} \mathrm{NMR}\left(75 \mathrm{MHz}, \mathrm{CDCl}_{3}\right): \delta 14.2\left(\mathrm{SCH}_{3}\right), 114.9(\mathrm{C}-5), 158.8(\mathrm{C}-6), 159.2(\mathrm{C}-4), 173.2(\mathrm{C}-2)$, $190.7(C=\mathrm{O})$.

$\mathbb{I R}(\mathrm{KBr}): v_{\max } 1695,1564,1553,1420,1356,1319$.

MS (EI): $m / z$ (rel intensity) 278 (100), 214 (23), 125 (76).

HRMS (EI): Calcd for $\mathrm{C}_{11} \mathrm{H}_{10} \mathrm{~N}_{4} \mathrm{O}^{32} \mathrm{~S}_{2}\left(\mathrm{M}^{+}\right)$278.0296, found 278.0289 . 
(2-Chloro-4-methoxypyridin-3-yl)bis-[2-(methylsulfanyl)pyrimidin-4-yl] methanol 1.65<smiles>COc1ccnc(Cl)c1I</smiles>

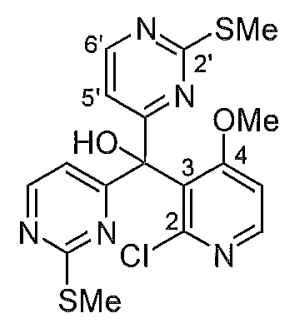

2-Chloro-4-methoxypyridine $(3.8,0.633 \mathrm{~g}, 4.41 \mathrm{mmol})$ was dissolved in freshly distilled THF $(18 \mathrm{~mL})$. The flask was cooled to below $-90{ }^{\circ} \mathrm{C}$ (acetone $/ \mathrm{N}_{2}$ ) and a solution of $n$-BuLi in hexanes (1.55 M, $2.9 \mathrm{~mL}, 4.5 \mathrm{mmol}$ ) was slowly added over $17 \mathrm{~min}$ to the stirred solution, keeping the temperature below $-90^{\circ} \mathrm{C}$. The orange solution was then stirred at $-78^{\circ} \mathrm{C}$ for $1 \mathrm{~h}$, by which time it had become a wine-red colour. The reaction mixture was again cooled to below $90^{\circ} \mathrm{C}$, and a solution of ketone $3.15(1.14 \mathrm{~g}, 4.09 \mathrm{mmol})$ in THF $(10 \mathrm{~mL})$ was added over $11 \mathrm{~min}$ while maintaining the temperature below $-90^{\circ} \mathrm{C}$. The dark mixture was then stirred at $-78^{\circ} \mathrm{C}$ for $3.5 \mathrm{~h}$, quenched with methanol and allowed to warm to $\mathrm{rt}$. The reaction mixture was shaken with sat aq $\mathrm{NH}_{4} \mathrm{Cl}$ solution, extracted with EtOAc and subjected to standard workup. Flash chromatography on silica, eluting with $80 \%$ EtOAc/hexanes, gave triarylmethanol 1.65 as a pale yellow solid (1.32 g, 76\%).

Mp: $135-137^{\circ} \mathrm{C}(t-\mathrm{BuOH})$.

${ }^{1} \mathrm{HI}$ NMR (500 MHz, $\left.\mathrm{CDCl}_{3}\right): \delta 2.49(\mathrm{~s}, 6 \mathrm{H}, \mathrm{SCH}), 3.43\left(\mathrm{~s}, 3 \mathrm{H}, \mathrm{OCH}_{3}\right), 6.55(\mathrm{~s}, 1 \mathrm{H}, \mathrm{OH}), 6.76$ (d, $J=5.4 \mathrm{~Hz}, 1 \mathrm{H}, \mathrm{H}-5$ ), 7.39 (d, $\left.J=5.4 \mathrm{~Hz}, 2 \mathrm{H}, \mathrm{H}-5^{\prime}\right), 8.25$ (d, $\left.J=5.4 \mathrm{~Hz}, 1 \mathrm{H}, \mathrm{H}-6\right), 8.46$ (d, $J$ $\left.=5.4 \mathrm{~Hz}, 2 \mathrm{H}, \mathrm{H}-6^{\prime}\right)$.

${ }^{13} \mathrm{C} \mathrm{NMR}\left(75 \mathrm{MHz}, \mathrm{CDCl}_{3}\right): \delta 14.1\left(\mathrm{SCH}_{3}\right), 55.8\left(\mathrm{OCH}_{3}\right), 78.0\left(\mathrm{Ar}_{3} \mathrm{COH}\right), 107.1(\mathrm{C}-5), 113.7$ (C-5'), 124.8 (C-3), 149.8 (C-6), 152.3 (C-2), 157.2 (C-6'), 165.9 (C-4), 171.0 (C-4'), 171.1 (C$\left.2^{\prime}\right)$.

$\mathbb{I R}\left(\mathrm{CDCl}_{3}\right): \mathrm{v}_{\max } 3344$ (br), 1576, 1551, 1348, 1209, 1043.

MS (EI): $m / z$ (rel intensity) 421/423 (65/29), 387 (19), 370 (21), 369 (21), 342 (38), 338 (57), 296 (44), 268 (60), 260 (100), 125 (98), 112 (22).

HRMS (EI): Calcd for $\mathrm{C}_{17} \mathrm{H}_{16}{ }^{35} \mathrm{ClN}_{5} \mathrm{O}_{2}{ }^{32} \mathrm{~S}_{2}\left(\mathrm{M}^{\dagger}\right)$ 421.0434, found 421.0448 . 
(2-Chlor - -4 -methoxypyridin-3-yl)bis-[2-(methylsulfanyl)pyrimidin-4-yll]methyl acetate 3.26<smiles>COc1nccc(C(=O)c2ccnc(SC)n2)n1</smiles><smiles>COc1ccnc(Cl)c1Cl</smiles><smiles>C[12CH2]</smiles><smiles>COc1nccc(C(O)(c2ccnc(S(C)(=O)=O)n2)c2c(OC)ccnc2Cl)n1</smiles>

2-Chloro-4-methoxypyridine $(3.8,0.141 \mathrm{~g}, 0.980 \mathrm{mmol})$ was dissolved in freshly distilled THF $(4 \mathrm{~mL})$. The flask was cooled to below $-90^{\circ} \mathrm{C}$ and a solution of $n$-BuLi in hexanes $(1.6 \mathrm{M}, 0.61$ $\mathrm{mL}, 0.98 \mathrm{mmol}$ ) was added over $2 \mathrm{~min}$ to the stirred solution. The orange solution was then stirred at $-78^{\circ} \mathrm{C}$ for $1 \mathrm{~h}$, by which time it had become a wine-red colour. The reaction mixture was again cooled to below $-90^{\circ} \mathrm{C}$ and a solution of ketone $3.15(271 \mathrm{mg}, 0.974 \mathrm{mmol})$ in $\mathrm{THF}$ $(2.2 \mathrm{~mL})$ was added over $2 \mathrm{~min}$. The dark mixture was stirred at $-78{ }^{\circ} \mathrm{C}$ for $3 \mathrm{~h}$, whereupon, freshly distilled acetyl chloride $(0.30 \mathrm{~mL}, 4.2 \mathrm{mmol})$ was added. After warming to rt overnight, the reaction mixture was shaken with sat aq $\mathrm{NaHCO}_{3}$ solution, extracted with EtOAc and subjected to standard workup. Flash chromatography on silica, eluting with $80 \%$ EtOAc/hexanes, gave acetate $\mathbf{3 . 2 6}(308 \mathrm{mg}, 68 \%$ ).

Mp: $151-154{ }^{\circ} \mathrm{C}(t-\mathrm{BuOH})$.

${ }^{1} \mathrm{H}$ NMR $\left(500 \mathrm{MHz}, \mathrm{CDCl}_{3}\right): \delta 2.30\left(\mathrm{~s}, 3 \mathrm{H}, \mathrm{OCOCH}_{3}\right), 2.39\left(\mathrm{~s}, 6 \mathrm{H}, \mathrm{SCH} \mathrm{OCH}_{3}\right), 3.49\left(\mathrm{~s}, 3 \mathrm{H}, \mathrm{OCH}_{3}\right)$, $6.76(\mathrm{~d}, J=5.6 \mathrm{~Hz}, 1 \mathrm{H}, \mathrm{H}-5), 7.18$ (d, $\left.J=5.4 \mathrm{~Hz}, 2 \mathrm{H}, \mathrm{H}-5^{\prime}\right), 8.23$ (d, $J=5.6 \mathrm{~Hz}, 1 \mathrm{H}, \mathrm{H}-6$ ), 8.39 (d, $\left.J=5.4 \mathrm{~Hz}, 2 \mathrm{H}, \mathrm{H}-6^{\prime}\right)$.

${ }^{13} \mathrm{C}$ NMR $\left(126 \mathrm{MHz}, \mathrm{CDCl}_{3}\right)$ : $\delta 14.0\left(\mathrm{SCH}_{3}\right), 21.4$ (OCOCH3), $55.9\left(\mathrm{OCH}_{3}\right), 84.5\left(\mathrm{Ar}_{3} \mathrm{COAc}\right)$, 107.0 (C-5), 114.8 (C-5'), 122.5 (C-3), 149.7 (C-6), 152.1 (C-2), 156.6 (C-6'), 166.3 (C-4), $168.16\left(\mathrm{C}-4^{\prime}\right.$ or $\left.C=\mathrm{O}\right), 168.22\left(\mathrm{C}=\mathrm{O}\right.$ or $\left.\mathrm{C}-4^{\prime}\right), 171.7\left(\mathrm{C}-2^{\prime}\right)$.

$\operatorname{IR}(\mathrm{KBr}): \mathrm{v}_{\max } 1755,1572,1549,1346,1207,1038$.

MS (EI): $m / z$ (rel intensity) 463(2), 428 (51), $423 / 421$ (61/27), 420 (55), 404 (24), 370 (37), 369 (100), $268(32), 125$ (48), 83 (31).

HRMS (EI): Calcd for $\mathrm{C}_{19} \mathrm{H}_{18} \mathrm{~N}_{5} \mathrm{O}_{3}{ }^{32} \mathrm{~S}_{2}\left(\mathrm{M}^{+}-\mathrm{Cl}\right)$ 428.0851, found 428.0860. 
4-Methoxy-9-(methylsulfanyl)-5-[2-(methylsulfamyl)pyrimidin-4-yl]pyrido $\left[3^{\prime}, 2^{\prime}: 4,5\right]$ pyrrolo [1,2-c]pyrimidine 1.63<smiles>[R]C(c1ccnc(OC)n1)(c1ccnc(SC)n1)c1c(C)ccnc1Cl</smiles>

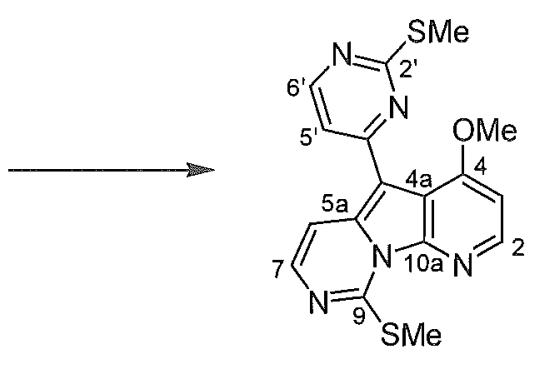

$\mathrm{R}=\mathrm{H}$ or $\mathrm{Ac}$

i) preparation from triarylmethanol 1.65

A mixture of triarylmethanol $1.65(100 \mathrm{mg}, 0.237 \mathrm{mmol})$ and TFA $(37 \mu \mathrm{L}, 0.48 \mathrm{mmol})$ was dissolved in 1,2-dichloroethane $(0.5 \mathrm{~mL})$. The resulting orange solution was transferred to a Young's tube, fitted with a rubber septum, containing TES $(0.30 \mathrm{~mL}, 1.9 \mathrm{mmol})$. With a strong stream of argon flowing, the septum was replaced with a teflon screw-cap, and the sealed reaction vessel was heated at $100{ }^{\circ} \mathrm{C}$ for $43 \mathrm{~h}$. After cooling, the vessel was opened and the contents diluted with $\mathrm{CH}_{2} \mathrm{Cl}_{2}(12 \mathrm{~mL})$. The solution was neutralised with $5 \%$ aq $\mathrm{NaHCO}_{3}$ solution $(8 \mathrm{~mL})$ and worked up with $\mathrm{CH}_{2} \mathrm{Cl}_{2}$ according to the standard procedure. Flash chromatography on silica, eluting with $48-75 \%$ EtOAc/hexanes, gave in order of elution

\section{a) 4-Methoxy-9-(methylsulfanyl)-5-[2-(methylsulfanyl)pyrimidin-4-yl]pyrido[3',2':4,5] pyrrolo[1,2-c]pyrimidine 1.63 as a yellow solid (41 mg, 47\%).}

Mp: $192-194^{\circ} \mathrm{C}$.

${ }^{1} \mathrm{H}$ NMR $\left(500 \mathrm{MHz}, \mathrm{CDCl}_{3}\right): \delta 2.65(\mathrm{~s}, 3 \mathrm{H}, 2$ '-SCH$), 2.70\left(\mathrm{~s}, 3 \mathrm{H}, 9-\mathrm{SCH}_{3}\right), 4.03\left(\mathrm{~s}, 3 \mathrm{H}, \mathrm{OCH}_{3}\right)$, $6.92(\mathrm{~d}, J=5.4 \mathrm{~Hz}, 1 \mathrm{H}, \mathrm{H}-3), 7.40$ (d, $\left.J=5.4 \mathrm{~Hz}, 1 \mathrm{H}, \mathrm{H}-5^{\prime}\right), 7.71$ (d, $J=6.8 \mathrm{~Hz}, 1 \mathrm{H}, \mathrm{H}-7$ ), 7.98 (d, $J=6.8 \mathrm{~Hz}, 1 \mathrm{H}, \mathrm{H}-6), 8.48\left(\mathrm{~m}, 2 \mathrm{H}, \mathrm{H}-2, \mathrm{H}-6^{\prime}\right)$.

${ }^{13} \mathrm{C}$ NMR $\left(75 \mathrm{MHz}, \mathrm{CDCl}_{3}\right)$ : $\delta 14.1\left(\mathrm{SCH}_{3}\right), 14.9\left(\mathrm{SCH}_{3}\right), 55.6\left(\mathrm{OCH}_{3}\right), 101.9(\mathrm{C}-3), 102.5(\mathrm{C}-$ 5), 108.5 (C-6), 110.8 (C-4a), 117.7 (C-5'), 135.9 (C-5a), 138.6 (C-7), 143.5 (C-2), 144.3 (C10a), 154.2 (C-9), 155.6 (C-6'), 159.6 (C-4), 161.1 (C-4'), 171.2 (C-2').

IR $\left(\mathrm{CDCl}_{3}\right): v_{\max } 1570,1468,1285,1013$.

MS (EI): $m / z$ (rel intensity) 369 (100), 354 (13), 112 (10), 70 (10).

HRMS (EI): Calcd for $\mathrm{C}_{17} \mathrm{H}_{15} \mathrm{~N}_{5} \mathrm{O}^{32} \mathrm{~S}_{2}\left(\mathrm{M}^{+}\right) 369.0718$, found 369.0720 . 
b) (2-Chloro-4-methoxypyridim-3-yl)-[2-(methylsulfanyl)pyrimidin-4-yl]-[2-(methylsulfanyl)pyrimidim-4-yloxy]methane 3.25 as a viscous gum (28 $\mathrm{mg}, 28 \%$ ). Trituration with $n$-hexane gave an off-white solid.

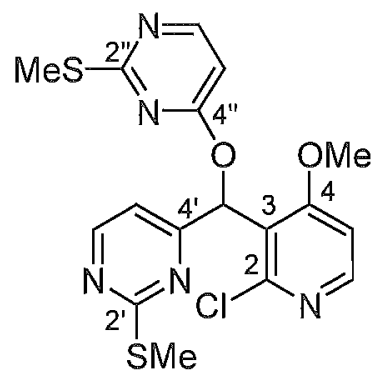

Mp: $113-121^{\circ} \mathrm{C}$.

${ }^{1} \mathbf{H} \mathbf{N M} \mathbb{R}\left(500 \mathrm{MHz}, \mathrm{CDCl}_{3}\right): \delta 2.36\left(\mathrm{~s}, 3 \mathrm{H}, 2^{\prime}-\mathrm{SCH} \mathrm{SH}_{3}\right), 2.44\left(\mathrm{~s}, 3 \mathrm{H}, 2 "-\mathrm{SCH}_{3}\right), 3.84\left(\mathrm{~s}, 3 \mathrm{H}, \mathrm{OCH}_{3}\right)$ 6.57 (d, $J=5.9 \mathrm{~Hz}, 1 \mathrm{H}, \mathrm{H}-5 "), 6.80$ (d, $J=5.9 \mathrm{~Hz}, 1 \mathrm{H}, \mathrm{H}-5$ ), 7.17 (d, $\left.J=4.9 \mathrm{~Hz}, 1 \mathrm{H}, \mathrm{H}-5^{\prime}\right), 7.79$ (s, $1 \mathrm{H}, \mathrm{Ar}_{2} \mathrm{CHOAr}$ ), 8.27 (d, $\left.J=5.9 \mathrm{~Hz}, 1 \mathrm{H}, \mathrm{H}-6\right), 8.30$ (d, $\left.J=5.9 \mathrm{~Hz}, 1 \mathrm{H}, \mathrm{H}-6 "\right), 8.49$ (d, $J=$ $\left.4.9 \mathrm{~Hz}, 1 \mathrm{H}, \mathrm{H}-6^{\prime}\right)$.

${ }^{13} \mathrm{C}$ NMR $\left(75 \mathrm{MHz}, \mathrm{CDCl}_{3}\right): \delta 13.88\left(\mathrm{SCH}_{3}\right), 13.90\left(\mathrm{SCH}_{3}\right), 56.2\left(\mathrm{OCH}_{3}\right), 71.8\left(\mathrm{Ar}_{2} \mathrm{CHOAr}\right)$, 103.5 (C-5"), 106.4 (C-5), 112.9 (C-5'), 120.8 (C-3), 150.7 (C-6), 152.5 (C-2), 157.1 (C-6'), 158.0 (C-6"), 166.0 (C-4), 167.1 (C-4'), 167.3 (C-4"), 172.1 (C-2'), 172.4 (C-2").

MS (EI): $m / z$ (rel intensity) 421/423 (94/43), 387 (21), 374/376 (33/13), 296 (79), 280 (39), 246 (79), 212 (100), 184 (28), 147 (43), 125 (58), 112 (45), 104 (48), 77 (53), 76 (50), 70 (44).

HRMS (EI): Calcd for $\mathrm{C}_{17} \mathrm{H}_{16}{ }^{35} \mathrm{ClN}_{5} \mathrm{O}_{2}{ }^{32} \mathrm{~S}_{2}\left(\mathrm{M}^{+}\right) 421.0434$, found 421.0444 .

ii) preparation from acetate 3.26

A mixture of acetate $3.26(112 \mathrm{mg}, 0.241 \mathrm{mmol})$ and TFA $(37 \mu \mathrm{L}, 0.48 \mathrm{mmol})$ was dissolved in 1,2-dichloroethane $(0.5 \mathrm{~mL})$. The resulting orange solution was transferred to a Young's tube, fitted with a rubber septum, containing TES $(0.31 \mathrm{~mL}, 1.9 \mathrm{mmol})$. With a strong stream of argon flowing, the septum was replaced with a teflon screw-cap, and the sealed reaction vessel was heated at $80^{\circ} \mathrm{C}$ for $26 \mathrm{~h}$. After cooling, the vessel was opened and the contents diluted with $\mathrm{CH}_{2} \mathrm{Cl}_{2}(15 \mathrm{~mL})$. The solution was neutralised with $5 \%$ aq $\mathrm{NaHCO}_{3}$ solution $(8 \mathrm{~mL})$ and the phases separated. The aqueous layer was further extracted with $\mathrm{CH}_{2} \mathrm{Cl}_{2}$ and the organic extracts were worked up according to the standard procedure. Flash chromatography on silica, eluting with $48 \%$ EtOAc/hexanes, gave core structure 1.63 (67 mg, 75\%). 
6-Acetoxy-4-methoxy-9-(methylsulfanyl)-5-[2-(methylsulfanyl)pyrimidin-4-yl] $]-6,7-$ dihydropyrido $\left[3^{\prime}, 2^{\prime}: 4,5\right]$ pyrrolo[1,2-c]pyrimidine 3.28
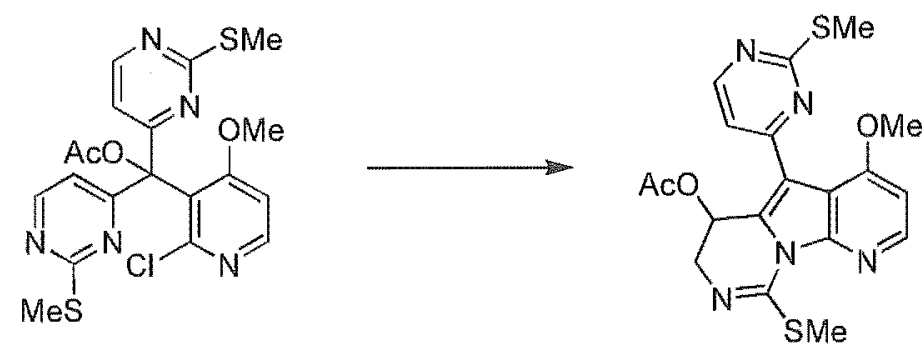

A mixture of acetate $3.26(110 \mathrm{mg}, 0.233 \mathrm{mmol}), \mathrm{HOAc}(30 \mu \mathrm{L}, 0.52 \mathrm{mmol})$ and TES $(80 \mu \mathrm{L}$, $0.50 \mathrm{mmol}$ ) was heated at reflux in 1,2-dichloroethane $(0.56 \mathrm{~mL})$ for $18 \mathrm{~h}$. After cooling, the reaction mixture was diluted with $\mathrm{CH}_{2} \mathrm{Cl}_{2}(15 \mathrm{~mL})$ and neutralised with $5 \%$ aq $\mathrm{NaHCO}_{3}$ solution ( $8 \mathrm{~mL}$ ). After further extraction with $\mathrm{CH}_{2} \mathrm{Cl}_{2}$, the organic extracts were worked up according to the standard procedure to give the crude product, which contained 1.63 as the major component. A minor product, the cyclised acetate 3.28 , was isolated after extensive flash chromatography on silica, eluting with $15 \% \mathrm{EtOAc} / \mathrm{CH}_{2} \mathrm{Cl}_{2}$ then $60 \% \mathrm{EtOAc} /$ hexanes, followed by reversed phase silica, eluting with $80-90 \% \mathrm{MeOH} /$ water, of sufficient purity for characterisation by ${ }^{1} \mathrm{H},{ }^{13} \mathrm{C}$ and 2D NMR spectroscopy.

${ }^{1}{ }_{H}$ NMR $\left(500 \mathrm{MHz}, \mathrm{CDCl}_{3}\right): 82.03\left(\mathrm{~s}, 3 \mathrm{H}, \mathrm{OCOCH}_{3}\right), 2.54\left(\mathrm{~s}, 3 \mathrm{H}, 9-\mathrm{SCH}_{3}\right), 2.58(\mathrm{~s}, 3 \mathrm{H}, 2$ '$\left.\mathrm{SCH} H_{3}\right), 3.66\left(\mathrm{dd}, J=3.4,17.0 \mathrm{~Hz}, 1 \mathrm{H}, \mathrm{H}-7_{\mathrm{a}}\right), 3.92\left(\mathrm{~s}, 3 \mathrm{H}, \mathrm{OCH}_{3}\right) 4.33(\mathrm{dd}, J=1.7,17.0 \mathrm{~Hz}, 1 \mathrm{H}$, H-7b), 6.50 (dd, $J=1.7,3.4 \mathrm{~Hz}, 1 \mathrm{H}, \mathrm{H}-6), 6.75$ (d, $J=5.4 \mathrm{~Hz}, 1 \mathrm{H}, \mathrm{H}-3), 7.30$ (d, $J=4.9 \mathrm{~Hz}, 1 \mathrm{H}$, H-5'), 8.40 (d, $J=5.4 \mathrm{~Hz}, 1 \mathrm{H}, \mathrm{H}-2), 8.53$ (d, $\left.J=4.9 \mathrm{~Hz}, 1 \mathrm{H}, \mathrm{H}-6^{\prime}\right)$.

${ }^{13} \mathrm{C} \mathrm{NMR}\left(126 \mathrm{MHz}, \mathrm{CDCl}_{3}\right): \delta 14.0\left(\mathrm{SCH}_{3}\right), 14.4\left(\mathrm{SCH}_{3}\right), 21.1\left(\mathrm{OCOCH}_{3}\right), 50.2(\mathrm{C}-7), 55.5$ $\left(\mathrm{OCH}_{3}\right), 61.3$ (C-6), 101.2 (C-3), 108.2 (C-4a), 113.1 (C-5), 117.9 (C-5'), 130.6 (C-5a), 146.8 (C-2), 147.7 (C-10a), 150.1 (br, C-9), 156.0 (C-6'), 159.7 (C-4'), 160.9 (C-4), 169.7 (C=O), $172.0\left(\mathrm{C}-2^{\prime}\right)$.

$\operatorname{IR}\left(\mathrm{CDCl}_{3}\right): \mathrm{v}_{\max } 1738$.

HRMS (ES): Calcd for $\mathrm{C}_{19} \mathrm{H}_{20} \mathrm{~N}_{5} \mathrm{O}_{3}{ }^{32} \mathrm{~S}_{2}\left(\mathrm{MH}^{+}\right)$430.1008, found 430.1010 . 
4-Methoxy-5-[2-(methylsulfanyl)pyrimidin-4-yl]-9-sulfanylpyrido [3',2':4,5]pyrrolo[1,2-c] pyrimidine 3.31
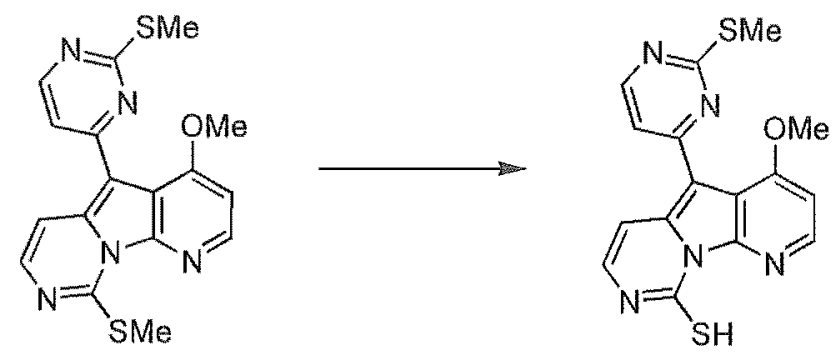

Core structure $1.63(9.1 \mathrm{mg}, 0.025 \mathrm{mmol})$ was dissolved in $\mathrm{CH}_{2} \mathrm{Cl}_{2}(1 \mathrm{~mL})$ and cooled to $-75^{\circ} \mathrm{C}$. A solution of boron tribromide in $\mathrm{CH}_{2} \mathrm{Cl}_{2}(\sim 1 \mathrm{M}, 0.25 \mathrm{~mL}, 0.25 \mathrm{mmol})$ was added to the cooled solution with stirring, causing a colour change from yellow to orange. After $1 \mathrm{~h}$ at $-75^{\circ} \mathrm{C}$, the reaction was allowed to warm to $\mathrm{rt}$, resulting in the formation of an orange precipitate. A small aliquot was worked up and shown by TLC to contain predominantly unreacted starting material. Therefore, 1,2-dichloroethane $(1 \mathrm{~mL})$ was added and the suspension was heated at $65^{\circ} \mathrm{C}$ for $4 \mathrm{~h}$. After cooling and dilution with $\mathrm{CH}_{2} \mathrm{Cl}_{2}$, water was added. The orange precipitate, which was not soluble in either phase, was filtered and washed with water and $\mathrm{CH}_{2} \mathrm{Cl}_{2}$ to give thiol 3.31 (3.7 $\mathrm{mg}, 42 \%)$.

${ }^{1} \mathrm{H}$ NMR $\left(500 \mathrm{MHz}, \mathrm{CD}_{3} \mathrm{OD}\right): \delta 2.60(\mathrm{~s}, 3 \mathrm{H}), 4.14(\mathrm{~s}, 3 \mathrm{H}), 7.12(\mathrm{~d}, J=7.6 \mathrm{~Hz}, 1 \mathrm{H}), 7.38-7.41$ $(\mathrm{m}, 2 \mathrm{H}), 7.43(\mathrm{~d}, J=7.6 \mathrm{~Hz}, 1 \mathrm{H}), 8.51(\mathrm{~d}, J=6.3 \mathrm{~Hz}, 1 \mathrm{H}), 8.55(\mathrm{~d}, J=5.4 \mathrm{~Hz}, 1 \mathrm{H}) ;(500 \mathrm{MHz}$, $\left.\left.\left(\mathrm{CD}_{3}\right)_{2} \mathrm{SO}\right): \delta 2.56(\mathrm{~s}, 3 \mathrm{H}, \mathrm{SCH})_{3}\right), 4.04\left(\mathrm{~s}, 3 \mathrm{H}, \mathrm{OCH}_{3}\right), 7.23(\mathrm{~d}, J=7.4 \mathrm{~Hz}, \mathrm{H}-7), 7.37(\mathrm{br}, 1 \mathrm{H}, \mathrm{H}-$ 3), 7.41-7.43 (m, 2H, H-6, H-5'), 8.54 (br, 1H, H-2), 8.63 (d, $J=5.2 \mathrm{~Hz}, \mathrm{H}-6$ '), 13.0 (br, 1H, $\mathrm{SH})$.

${ }^{13} \mathrm{C} \mathrm{NMR}\left(75 \mathrm{MHz},\left(\mathrm{CD}_{3}\right)_{2} \mathrm{SO}\right): \delta 13.7\left(\mathrm{SCH}_{3}\right), 56.8\left(\mathrm{OCH}_{3}\right), 101.6(\mathrm{C}-6), 104.1(\mathrm{C}-3), 104.9$ (C-5), 112.3 (C-4a), 118.6 (C-5'), 128.5 (C-7), 134.2 (C-5a), 142.0 (C-2), 143.9 (br, C-10a), $156.7\left(\mathrm{C}-6^{\prime}\right), 159.6\left(\mathrm{C}-4^{\prime}\right), 161.4(\mathrm{C}-4), 168.6(\mathrm{C}-9), 170.6\left(\mathrm{C}-2^{\prime}\right)$.

HRMS (ES): Calcd for $\mathrm{C}_{16} \mathrm{H}_{14} \mathrm{~N}_{5} \mathrm{O}^{32} \mathrm{~S}_{2}\left(\mathrm{MH}^{+}\right) 356.0640$, found 356.0647 . 
4-Methoxy-9-(methylsulfinyl)-5-[2-(methylsulfinyl)pyrimidin-4-yl]pyrido[3',2':4,5]pyrrolo $[1,2-c]$ pyrimidine 3.32<smiles>COc1ccnc2c1c(-c1ccnc(SC)n1)c1ccnc(SC)n12</smiles><smiles>COc1ccnc2c1c(-c1ccnc([As](C)=O)n1)c1ccnc([O-])n12</smiles>

Core structure $1.63(37 \mathrm{mg}, 0.10 \mathrm{mmol})$ was dissolved in $\mathrm{CHCl}_{3}(5 \mathrm{~mL})$ under atmospheric conditions and cooled to $-40{ }^{\circ} \mathrm{C}\left(\mathrm{CH}_{3} \mathrm{CN} / \mathrm{CO}_{2}\right)$. A solution of $m$-CPBA in $\mathrm{CHCl}_{3}(10 \mathrm{mg} / \mathrm{mL})$ was similarly cooled and added dropwise to the solution of mve until TLC analysis indicated the consumption of all starting material (after about 2 equiv of $m$-CPBA). The solution was warmed to $\mathrm{rt}$ and neutralised with sat aq $\mathrm{NaHCO}_{3}$ solution. This was extracted with $\mathrm{CH}_{2} \mathrm{Cl}_{2}$ and standard workup gave a yellow solid which was predominantly a mixture of diastereomeric bis-sulfoxides 3.32. The crude mixture was used without purification, however the bis-sulfoxides had the following spectroscopic characteristics:

${ }^{1} \mathrm{H}$ NMR $\left(500 \mathrm{MHz}, \mathrm{CDCl}_{3}\right.$ ): (As most signals for the diastereoisomers coincide, they are all quoted as multiplets.) $\delta 3.02(\mathrm{~m}, 3 \mathrm{H}), 3.19(\mathrm{~m}, 3 \mathrm{H}), 4.12(\mathrm{~m}, 3 \mathrm{H}), 7.00-7.01(\mathrm{~m}, 1 \mathrm{H}), 7.98-7.99$ $(\mathrm{m}, 1 \mathrm{H}), 8.12-8.14(\mathrm{~m}, 1 \mathrm{H}), 8.48-8.49(\mathrm{~m}, 1 \mathrm{H}), 8.64-8.67(\mathrm{~m}, 1 \mathrm{H}), 8.79-8.81(\mathrm{~m}, 1 \mathrm{H})$. 
4-Methoxy-9-(4-methoxybenzylamino)-5-[2-(4-methoxybenzylamimo)pyrimidin-4-yllpyrido $\left[3^{\prime}, 2^{\prime}: 4,5\right]$ pyrrolo $[1,2-c \mid$ pyrimidine 3.34
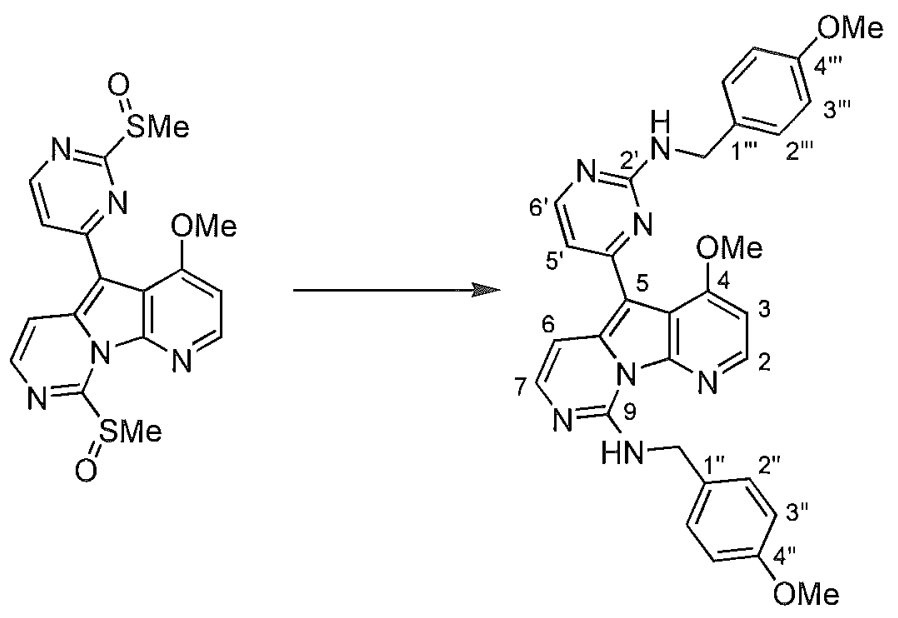

The crude oxidised material 3.32 was heated with an excess of $p$-methoxybenzylamine $(0.15 \mathrm{~mL}$, $1.1 \mathrm{mmol}$ ) at $85^{\circ} \mathrm{C}$ for $15 \mathrm{~h}$. The red paste thus obtained was directly purified by flash chromatography on silica, eluting with $2.5-4 \% \mathrm{MeOH} / \mathrm{CH}_{2} \mathrm{Cl}_{2}$. This was chromatographed again on silica, eluting with $50 \% \mathrm{EtOAc} / \mathrm{CH}_{2} \mathrm{Cl}_{2}$ then $100 \%$ EtOAc, to give bis-amine 3.34 as a yellow-orange solid ( $43 \mathrm{mg}, 78 \%$ over two steps).

Mp: $74-77^{\circ} \mathrm{C}$.

${ }^{1} \mathrm{H}$ NMR $\left(500 \mathrm{MHz}, \mathrm{CDCl}_{3}\right): \delta 3.81(\mathrm{~s}, 6 \mathrm{H}$, both PMB OCH$), 3.99\left(\mathrm{~s}, 3 \mathrm{H}, 4-\mathrm{OCH}_{3}\right), 4.66(\mathrm{~d}, J$ $\left.=5.6 \mathrm{~Hz}, 2 \mathrm{H}, 2^{\prime}-\mathrm{NHCH}_{2} \mathrm{Ar}\right), 4.85$ (d, $J=5.5 \mathrm{~Hz}, 2 \mathrm{H}, 9-\mathrm{NHCH}_{2} \mathrm{Ar}$ ), 5.51 (br, $1 \mathrm{H}, 2^{\prime}-\mathrm{N} H \mathrm{PMB}$ ), 6.82 (d, $J=5.6$ Hz, 1H, H-3), 6.89-6.91 (m, 4H, PMB H-3", PMB H-3"'), 7.00 (d, $J=5.2 \mathrm{~Hz}$, 1H, H-5'), 7.29 (br, 1H, H-6), 7.34 (d, $\left.J=8.5 \mathrm{~Hz}, 2 \mathrm{H}, \mathrm{PMB} \mathrm{H}-2^{\prime \prime \prime}\right), 7.39$ (d, $J=8.5 \mathrm{~Hz}, 2 \mathrm{H}, \mathrm{PMB}$ H-2"), 7.43 (br d, $J=6.3 \mathrm{~Hz}, 1 \mathrm{H}, \mathrm{H}-7$ ), 8.16 (d, $J=5.6 \mathrm{~Hz}, 1 \mathrm{H}, \mathrm{H}-2$ ), 8.26 (d, $J=5.2 \mathrm{~Hz}, 1 \mathrm{H}, \mathrm{H}-$ 6'), 10.39 (br t, $J=5.5 \mathrm{~Hz}, 1 \mathrm{H}, 9-\mathrm{N} H \mathrm{PMB}$ ).

${ }^{13} \mathrm{C}$ NMR $\left(75 \mathrm{MHz}, \mathrm{CDCl}_{3}\right): \delta 44.3\left(9-\mathrm{NHCH}_{2} \mathrm{Ar}\right), 44.9\left(2^{\prime}-\mathrm{NHCH}_{2} \mathrm{Ar}\right), 55.3$ (both PMB $\left.\mathrm{OCH}_{3}\right), 55.5\left(4-\mathrm{OCH}_{3}\right), 101.3(\mathrm{C}-5), 101.6(\mathrm{C}-3), 101.8(\mathrm{C}-6), 111.4(\mathrm{C}-4 \mathrm{a}), 112.2\left(\mathrm{C}-5^{\prime}\right), 113.96$ (PMB C-3"' or PMB C-3"), 114.03 (PMB C-3" or PMB C-3"'), 128.5 (PMB C-2"'), 128.8 (PMB C-2"), 130.5 (PMB C-1"), 131.4 (PMB C-1"'), 137.5 (C-5a), 141.5 (C-2), 141.9 (C-7), 144.7 (C10a), 148.7 (C-9), 154.6 (br, C-6'), 158.7 (PMB C-4'"), 158.9 (PMB C-4"), 159.4 (C-4), 160.9 $\left(\mathrm{C}-2^{\prime}\right), 162.6\left(\mathrm{C}-4^{\prime}\right)$.

IR (KBr): $v_{\max } 3242,2997-2833$ (series of weak bands), 1570. 
MS (EI): $m / z$ (rel intensity) 547 (65), 427 (14), 426 (9), 425 (12), 136 (38), 135 (53), 121 (100), $112(21), 77(24)$.

HRMS (EI): Calcd for $\mathrm{C}_{31} \mathrm{H}_{29} \mathrm{~N}_{7} \mathrm{O}_{3}\left(\mathrm{M}^{+}\right)$547.2332, found 547.2334.

\section{4-Hydroxy-9-(4-methoxybenzylamino)-5-[2-(4-methoxybenzylamino)pyrimidin-4-yl]pyrido} $\left[3^{\prime}, 2^{\prime}: 4,5\right]$ pyrrolo $[1,2-c]$ pyrimidine 3.35
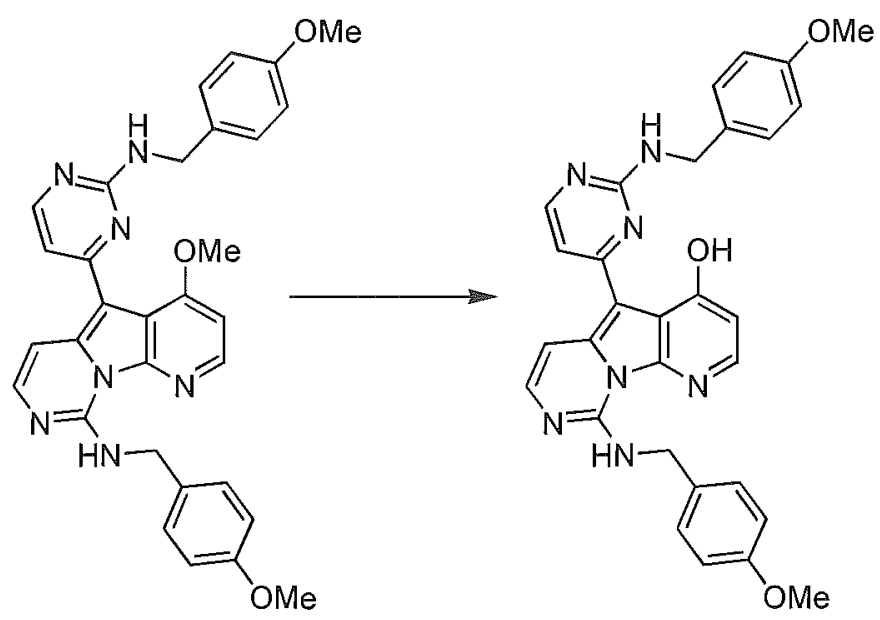

A solution of sodium ethanethiolate $(80 \%, 202 \mathrm{mg}, 1.92 \mathrm{mmol})$ in dry DMF $(2 \mathrm{~mL})$ was added to a solution of bis-amine 3.34 (106 $\mathrm{mg}, 0.193 \mathrm{mmol})$ in DMF (2 $\mathrm{mL})$, and the mixture was stirred at $55^{\circ} \mathrm{C}$ for $7.5 \mathrm{~h}$. After cooling, aq $\mathrm{NH}_{4} \mathrm{Cl}$ solution was added and the mixture was extracted with EtOAc. The organic extracts were washed three times with water to remove DMF and worked up as usual. Flash chromatography on silica, eluting with $4 \% \mathrm{MeOH} / \mathrm{CH}_{2} \mathrm{Cl}_{2}$, gave pyridinol 3.35 as a yellow solid (98 $\mathrm{mg}, 95 \%)$.

Mp: $195-196{ }^{\circ} \mathrm{C}\left(\mathrm{CH}_{2} \mathrm{Cl}_{2} / \mathrm{MeOH}\right)$.

${ }^{1} \mathrm{H}$ NMR $\left(500 \mathrm{MHz}, \mathrm{CDCl}_{3}\right): \delta 3.80\left(\mathrm{~s}, 3 \mathrm{H}, \mathrm{OCH}_{3}\right), 3.81\left(\mathrm{~s}, 3 \mathrm{H}, \mathrm{OCH}_{3}\right), 4.61(\mathrm{~d}, J=5.5 \mathrm{~Hz}, 2 \mathrm{H}$, $\left.2^{\prime}-\mathrm{NHCH} H_{2} \mathrm{Ar}\right), 4.85$ (d, $J=5.5 \mathrm{~Hz}, 2 \mathrm{H}, 9-\mathrm{NHCH}_{2} \mathrm{Ar}$ ), 5.35 (br, 1H, 2'-NHPMB), 6.76 (d, $J=5.5$ Hz, 1H, H-3), 6.88-6.92 (m, 4H, PMB H-3", PMB H-3"'), 7.03 (d, $J=6.8$ Hz, 1H, H-6), 7.06 (d, $\left.J=5.7 \mathrm{~Hz}, 1 \mathrm{H}, \mathrm{H}-5^{\prime}\right), 7.32$ (d, $\left.J=8.4 \mathrm{~Hz}, 2 \mathrm{H}, \mathrm{PMB} \mathrm{H}-2^{\prime \prime \prime}\right), 7.40$ (d, $\left.J=8.4 \mathrm{~Hz}, 2 \mathrm{H}, \mathrm{PMB} \mathrm{H}-2^{\prime \prime}\right)$, $7.68(\mathrm{~d}, J=6.8 \mathrm{~Hz}, 1 \mathrm{H}, \mathrm{H}-7), 8.05(\mathrm{~d}, J=5.5 \mathrm{~Hz}, 1 \mathrm{H}, \mathrm{H}-2), 8.29(\mathrm{~d}, J=5.7 \mathrm{~Hz}, 1 \mathrm{H}, \mathrm{H}-6), 10.94$ (br t, $J=5.5 \mathrm{~Hz}, 1 \mathrm{H}, 9-\mathrm{NHPMB}), 15.7(\mathrm{br}, 1 \mathrm{H}, \mathrm{OH}$ ). 
${ }^{13} \mathrm{C}$ NMR $\left(75 \mathrm{MHz}, \mathrm{CDCl}_{3}\right): 844.3\left(9-\mathrm{NHCH}_{2} \mathrm{Ar}\right), 45.2$ (2'-NHCH $2 \mathrm{Ar}$ ), 55.3 (both $\mathrm{OCH}_{3}$ ), 100.3 (C-6), 100.5 (C-5), 106.9 (C-5'), 107.5 (C-3), 111.4 (C-4a), 114.1 (PMB C-3", PMB C3"'), 128.8 (PMB C-2"), 129.2 (PMB C-2"'), 130.2 (PMB C-1", PMB C-1"'), 137.5 (C-5a), 142.8 (C-2), 143.8 (C-7), 145.4 (C-10a), 149.7 (C-9), 158.6 (C-6'), 158.9 (PMB C-4"), 159.0 (PMB C$\left.4^{\prime \prime \prime}\right), 159.5\left(\mathrm{C}-4^{\prime}\right), 159.8(\mathrm{C}-4), 160.0\left(\mathrm{C}-2^{\prime}\right)$.

IR (KBr): $v_{\max } 3223,2820-2835$ (series of weak bands), 1574.

MS (EI): $m / z$ (rel intensity) 533 (38), 413 (19), 412 (18), 411 (15), 121 (100).

HRMS (EI): Calcd for $\mathrm{C}_{30} \mathrm{H}_{27} \mathrm{~N}_{7} \mathrm{O}_{3}\left(\mathrm{M}^{\dagger}\right)$ 533.2175, found 533.2185 .

\section{Variolin B 1.17}
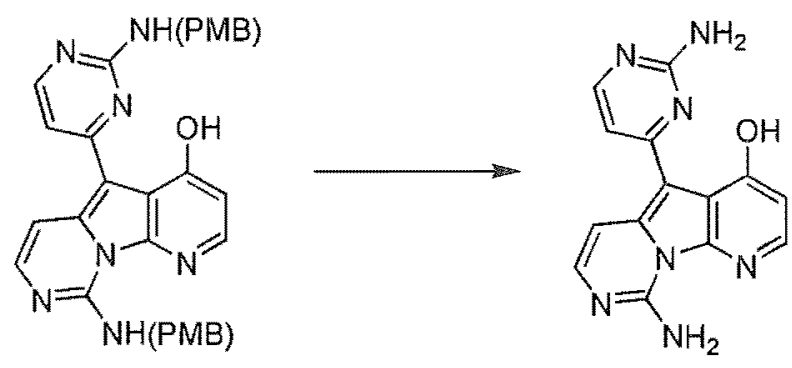

Pyridinol 3.35 (31 mg, $0.058 \mathrm{mmol})$ was dissolved in neat $\mathrm{TfOH}(0.5 \mathrm{~mL})$ under atmospheric conditions. The flask was stoppered and the deep red solution was left at $\mathrm{rt}$ for $17 \mathrm{~h}$. The reaction was cooled in ice and carefully quenched with ice-water $(1 \mathrm{~mL})$, followed by conc aq $\mathrm{NH}_{3}$ solution $(1 \mathrm{~mL})$ which produced a bright yellow precipitate. After mixing thoroughly to ensure complete neutralisation, the precipitate was filtered through a fine frit, washing with water and then $\mathrm{MeOH} /$ water (1:1). The solid was then suspended in $\mathrm{MeOH} / \mathrm{CH}_{2} \mathrm{Cl}_{2}$ (a small amount of TFA was added to convert the free base to the more soluble salt form) and adsorbed onto a small amount of reversed phase silica for flash chromatography, eluting with $50 \%$ $\mathrm{MeOH} /$ water then $70 \% \mathrm{MeOH} /$ water $+0.5 \% \mathrm{TFA}$. The bright yellow fractions were combined and concentrated in vacuo to give variolin B as its trifluoroacetate salt. The salt was neutralised by sonication with conc aq $\mathrm{NH}_{3} / \mathrm{MeOH}$ to give the free base. Concentration in vacuo $\left(35^{\circ} \mathrm{C}\right.$, $0.03 \mathrm{~mm} \mathrm{Hg}$ to remove ammonium trifluoroacetate) gave variolin B (1.17) as a brown-orange solid (13.6 mg, 80\%). 
Mp: dec at $\sim 320^{\circ} \mathrm{C}\left(\right.$ lit $^{32 \mathrm{a}} 45^{\circ} \mathrm{C}$, dec).

${ }^{1} \mathrm{H} \mathrm{NMR}\left(500 \mathrm{MHz},\left(\mathrm{CD}_{3}\right)_{2} \mathrm{SO}\right): \delta 6.81(\mathrm{~d}, J=5.6 \mathrm{~Hz}, 1 \mathrm{H}, \mathrm{H}-3), 6.97\left(\mathrm{~s}, 2 \mathrm{H}, 2^{\prime}-\mathrm{NH}_{2}\right), 7.14(\mathrm{~d}, J$ $\left.=5.6 \mathrm{~Hz}, 1 \mathrm{H}, \mathrm{H}-5^{\prime}\right), 7.23(\mathrm{~d}, J=6.7 \mathrm{~Hz}, 1 \mathrm{H}, \mathrm{H}-6), 7.63(\mathrm{~d}, J=6.7 \mathrm{~Hz}, \mathrm{H}-7), 8.17(\mathrm{~d}, J=5.6 \mathrm{~Hz}$, $1 \mathrm{H}, \mathrm{H}-2), 8.27\left(\mathrm{~d}, J=5.6 \mathrm{~Hz}, 1 \mathrm{H}, \mathrm{H}-6^{\prime}\right), 8.45(\mathrm{br}, 1 \mathrm{H}, 9-\mathrm{NH}), 9.79(\mathrm{br}, 1 \mathrm{H}, 9-\mathrm{N} H), 16.05(\mathrm{~s}, 1 \mathrm{H}$, $\mathrm{OH}$.

${ }^{13} \mathrm{C}$ NMR $\left(75 \mathrm{MHz},\left(\mathrm{CD}_{3}\right)_{2} \mathrm{SO}\right): \delta 99.6(\mathrm{C}-5), 100.3(\mathrm{C}-6), 106.0(\mathrm{C}-5), 107.5(\mathrm{C}-3), 111.2(\mathrm{C}-$ 4a), 137.1 (C-5a), 143.1 (C-2), 144.6 (C-7), 144.9 (C-10a), 150.3 (C-9), 158.3 (C-4'), 159.8 (C4), $160.0\left(\mathrm{C}-6^{\prime}\right), 161.4\left(\mathrm{C}-2^{\prime}\right)$.

$\mathbb{I R}(\mathrm{KBr}): \mathrm{U}_{\max } 3085,1670,1572,1477,1458,1298$.

HIRMS (ES): Calcd for $\mathrm{C}_{14} \mathrm{H}_{12} \mathrm{~N}_{7} \mathrm{O}\left(\mathrm{MH}^{+}\right)$294.1103, found 294.1096. 


\subsection{Experiments Described in Chapter Four}

\section{9-Amino-4-methoxy-5-(2-amin opyrimidin-4-yl)pyrido $\left[33^{\prime}, 2^{\prime}: 4,5\right]$ pyrrolo[1,2-c]pyrimidine}

4.1
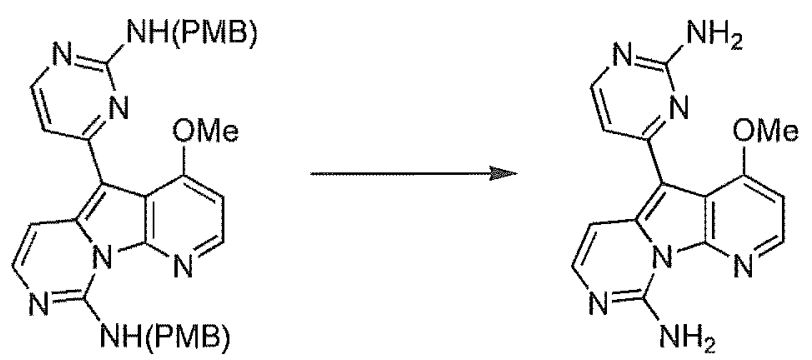

Bis-amine $3.34(10 \mathrm{mg}, 0.018 \mathrm{mmol})$ was dissolved in neat $\mathrm{TfOH}(0.1 \mathrm{~mL})$ in a small vial under atmospheric conditions. The vial was capped and the deep red solution was left at $\mathrm{rt}$ for $13 \mathrm{~h}$. The reaction was cooled to $-10^{\circ} \mathrm{C}$ and carefully quenched with $\mathrm{MeOH}(0.2 \mathrm{~mL})$, followed by conc aq $\mathrm{NH}_{3}$ solution $(0.1 \mathrm{~mL})$. After mixing thoroughly to ensure complete neutralisation, the precipitate was filtered through a $\mathrm{C}_{18}$ cartridge to remove ammonium triflate, washing with water and then $30 \% \mathrm{MeOH}$ /water. The yellow precipitate was then dissolved in $25 \% \mathrm{CH}_{2} \mathrm{Cl}_{2} / \mathrm{MeOH}+$ $0.2 \%$ TFA, and eluted through the cartridge to give, after concentration in vacuo, a yellow solid. Flash chromatography on silica, eluting with $5-8 \% \mathrm{MeOH} / \mathrm{CH}_{2} \mathrm{Cl}_{2}$ then $10 \% \mathrm{MeOH} / \mathrm{CH}_{2} \mathrm{Cl}_{2}+$ $0.5 \% \mathrm{NEt}_{3}$, followed by flash chromatography on reversed phase silica, eluting with 70,80 and then $90 \% \mathrm{MeOH} /$ water, gave $O$-methylvariolin $\mathrm{B}(4.1)$ as a yellow-brown solid $(1.3 \mathrm{mg}, 23 \%)$.

${ }^{1} \mathrm{H}$ NMR $\left(500 \mathrm{MHz},\left(\mathrm{CD}_{3}\right)_{2} \mathrm{SO}\right): \delta 4.02\left(\mathrm{~s}, 3 \mathrm{H}, \mathrm{OCH}_{3}\right), 6.43\left(\mathrm{~s}, 2 \mathrm{H}, 2^{\prime}-\mathrm{NH} H_{2}\right), 6.94(\mathrm{~d}, J=5.6 \mathrm{~Hz}$, $\left.1 \mathrm{H}, \mathrm{H}-5^{\prime}\right), 7.17$ (d, $\left.J=5.6 \mathrm{~Hz}, 1 \mathrm{H}, \mathrm{H}-3\right), 7.46$ (d, $\left.J=6.6 \mathrm{~Hz}, 1 \mathrm{H}, \mathrm{H}-7\right), 7.60$ (d, $J=6.6 \mathrm{~Hz}, 1 \mathrm{H}$, $\mathrm{H}-6), 8.17(\mathrm{br} \mathrm{d}, J=5.6 \mathrm{~Hz}, 1 \mathrm{H}, \mathrm{H}-6), 8.3(\mathrm{br}, 1 \mathrm{H}, 9-\mathrm{NH}), 8.34(\mathrm{~d}, J=5.6 \mathrm{~Hz}, 1 \mathrm{H}, \mathrm{H}-2), 9.35$ (br, $1 \mathrm{H}, 9-\mathrm{N} H$ ).

${ }^{13} \mathrm{C} \mathrm{NMR}\left(75 \mathrm{MHz}, \mathrm{CDCl}_{3}\right): \delta 56.1\left(\mathrm{OCH}_{3}\right), 100.7(\mathrm{C}-5), 101.8(\mathrm{C}-6), 102.7(\mathrm{C}-3), 110.9(\mathrm{C}-$ 4a), $111.5(\mathrm{C}-5), 136.4$ (C-5a), 141.7 (C-7), 142.2 (C-2), 143.9 (C-10a), 149.1 (C-9), 156.7 (C$\left.6^{\prime}\right), 159.3(\mathrm{C}-4), 161.4\left(\mathrm{C}-4^{\prime}\right.$ or $\left.\mathrm{C}-2^{\prime}\right), 163.2\left(\mathrm{C}-2^{\prime}\right.$ or $\left.\mathrm{C}-4^{\prime}\right)$.

IR (KBr): $\cup_{\max } 3320,2922,2856,1570$.

HRMS (ES): Calcd for $\mathrm{C}_{15} \mathrm{H}_{14} \mathrm{~N}_{7} \mathrm{O}\left(\mathrm{MH}^{+}\right)$308.1260, found 308.1256. 
4-Methoxy-9-(4-methoxybenzylamino)-5-[2-(methylsulfanyl)pyrimidin-4-yl]pyrido $\left[3^{\prime}, 2^{\prime}: 4,5\right]$ pyrrolo $[1,2-c]$ pyrimidine 4.9
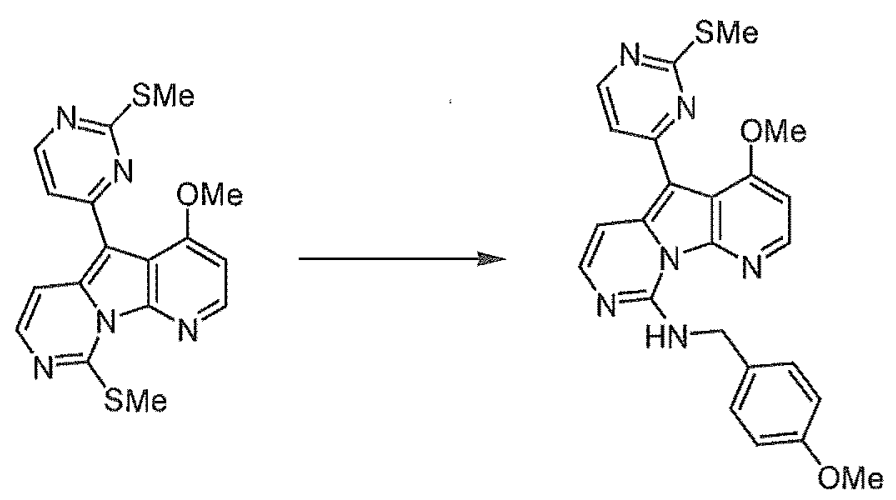

Core structure 1.63 ( $20 \mathrm{mg}, 0.054 \mathrm{mmol}$ ) was heated with an excess of $p$-methoxybenzylamine $(0.10 \mathrm{~mL}, 0.77 \mathrm{mmol})$ at $85^{\circ} \mathrm{C}$ for $15 \mathrm{~h}$. The red paste thus obtained was directly purified by flash chromatography on silica, eluting with $60-80 \%$ EtOAc/hexanes, to give protected amine 4.9 as a micro-crystalline yellow solid ( $21 \mathrm{mg}, 86 \%)$.

Mp: $173-175^{\circ} \mathrm{C}$.

${ }^{1}{ }_{H} \operatorname{NMR}\left(500 \mathrm{MHz}, \mathrm{CDCl}_{3}\right): \delta 2.65$ (s, 3H, SCH$), 3.81$ (s, 3H, PMB OCH $\left.H_{3}\right), 4.02(\mathrm{~s}, 3 \mathrm{H}, 4-$ $\left.\mathrm{OCH}_{3}\right), 4.87$ (d, $\left.J=5.2 \mathrm{~Hz}, 2 \mathrm{H}, \mathrm{NHCH}_{2} \mathrm{Ar}\right), 6.85$ (d, $\left.J=5.6 \mathrm{~Hz}, 1 \mathrm{H}, \mathrm{H}-3\right), 6.91$ (d, $J=8.5 \mathrm{~Hz}$, 2H, PMB H-3"), 7.37 (d, $\left.J=5.4 \mathrm{~Hz}, 1 \mathrm{H}, \mathrm{H}-5^{\prime}\right), 7.40$ (d, $\left.J=8.5 \mathrm{~Hz}, 2 \mathrm{H}, \mathrm{PMB} H-2^{\prime \prime}\right), 7.58$ (d, $J=$ $6.6 \mathrm{~Hz}, 1 \mathrm{H}, \mathrm{H}-6), 7.62$ (d, $J=6.6 \mathrm{~Hz}, 1 \mathrm{H}, \mathrm{H}-7), 8.19$ (d, $J=5.6 \mathrm{~Hz}, 1 \mathrm{H}, \mathrm{H}-2), 8.43$ (d, $J=5.4$ $\mathrm{Hz}, 1 \mathrm{H}, \mathrm{H}-6^{\prime}$ ), 10.43 (br t, $J=5.2 \mathrm{~Hz}, 1 \mathrm{H}, \mathrm{N} H \mathrm{PMB}$ ).

${ }^{13} \mathrm{C} \mathrm{NMR}\left(126 \mathrm{MHz}, \mathrm{CDCl}_{3}\right): \delta 14.1\left(\mathrm{SCH}_{3}\right), 44.4\left(\mathrm{NHCH}_{2} \mathrm{Ar}\right), 55.3\left(\mathrm{PMB} \mathrm{OCH}_{3}\right), 55.6$ (4$\mathrm{OCH}_{3}$ ), 100.7 (C-5), 101.6 (C-6 or C-3), 101.7 (C-3 or C-6), 111.3 (C-4a), 114.1 (PMB C-3"), 117.3 (C-5'), 128.8 (PMB C-2"), 130.4 (PMB C-1"), 137.7 (C-5a), 141.6 (C-2), 142.3 (C-7), 144.8 (C-10a), 148.7 (C-9), 155.4 (C-6'), 158.9 (PMB C-4"), 159.4 (C-4), 161.5 (C-4'), 171.1 (C2).

IR (KBr): $\mathrm{U}_{\max } 3238,3060-2836$ (series of weak bands), 1610, 1558 .

MS (EI): $m / z$ (rel intensity) 458 (77), 121 (100).

HRMS (EI): Calcd for $\left.\mathrm{C}_{24} \mathrm{H}_{22} \mathrm{~N}_{6} \mathrm{O}_{2}{ }^{32} \mathrm{~S}_{\left(\mathrm{M}^{+}\right)}\right) 458.1525$, found 458.1535 . 


\section{4-Hydroxy-9-(4-methoxybenzylamino)-5-[2-(methylsulfanyl)pyrimidin-4-yl]pyrido} $\left[3^{\prime}, 2^{\prime}: 4,5\right]$ pyrrolo $[1,2-c]$ pyrimidine 4.10
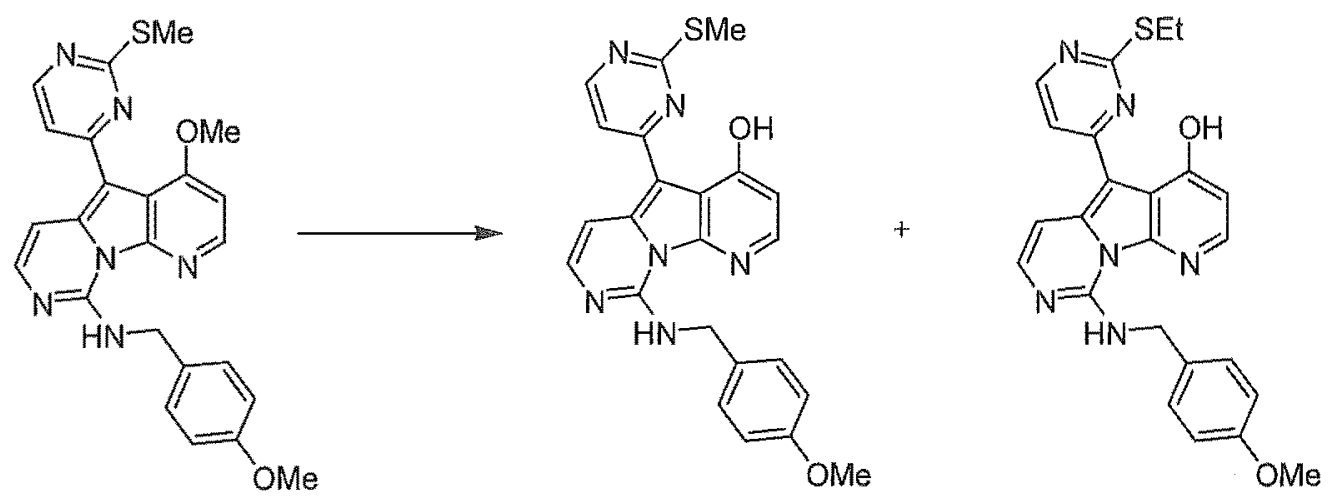

Sodium ethanethiolate $(80 \%, 144 \mathrm{mg}, 1.37 \mathrm{mmol})$ was dissolved in dry DMF $(1.4 \mathrm{~mL})$. A portion $(0.24 \mathrm{~mL}, 0.23 \mathrm{mmol})$ of this solution was added to a solution of amine 4.9 (10.7 mg, $23.3 \mu \mathrm{mol})$ in DMF $(0.24 \mathrm{~mL})$, and the mixture was stirred at $50^{\circ} \mathrm{C}$ for $12 \mathrm{~h}$. After cooling, aq $\mathrm{NH}_{4} \mathrm{Cl}$ solution was added, and the mixture was extracted with $\mathrm{CH}_{2} \mathrm{Cl}_{2}$ and worked up in the usual manner. Flash chromatography on silica, eluting with $2 \% \mathrm{MeOH} / \mathrm{CH}_{2} \mathrm{Cl}_{2}$, gave pyridinol 4.10 as a yellow solid, contaminated with trace amounts of the SEt-substituted analogue 4.11 (9.9 mg, 95\%).

Mp: $198^{\circ} \mathrm{C}$ (dec).

${ }^{1} \mathbf{H}$ NMR $\left(500 \mathrm{MHz}, \mathrm{CDCl}_{3}\right): \delta 2.64\left(\mathrm{~s}, 3 \mathrm{H}, \mathrm{SCH} H_{3}\right), 3.81$ (s, 3H, PMB OCH $), 4.85$ (d, $J=5.4$ $\mathrm{Hz}, 2 \mathrm{H}, \mathrm{NHCH}_{2} \mathrm{Ar}$ ), 6.84 (d, $J=5.6 \mathrm{~Hz}, 1 \mathrm{H}, \mathrm{H}-3$ ), 6.91 (d, $\left.J=8.9 \mathrm{~Hz}, 2 \mathrm{H}, \mathrm{PMB} \mathrm{H}-3 "\right), 7.01$ (d, $J=6.5 \mathrm{~Hz}, 1 \mathrm{H}, \mathrm{H}-6), 7.37$ (d, $\left.J=5.7 \mathrm{~Hz}, 1 \mathrm{H}, \mathrm{H}-5^{\prime}\right), 7.40$ (d, $J=8.9 \mathrm{~Hz}, 2 \mathrm{H}$, PMB H-2"), 7.73 (d, $J=6.5 \mathrm{~Hz}, 1 \mathrm{H}, \mathrm{H}-7), 8.06$ (d, $J=5.6 \mathrm{~Hz}, 1 \mathrm{H}, \mathrm{H}-2), 8.42$ (d, $J=5.7 \mathrm{~Hz}, 1 \mathrm{H}, \mathrm{H}-6$ ), 11.00 (br $\mathrm{t}, J=5.4 \mathrm{~Hz}, 1 \mathrm{H}, \mathrm{NHPMB}), 14.70(\mathrm{~s}, 1 \mathrm{H}, \mathrm{O} H)$.

${ }^{13} \mathrm{C} \mathrm{NMR}\left(75 \mathrm{MHz}, \mathrm{CDCl}_{3}\right): \delta 13.9\left(\mathrm{SCH}_{3}\right), 44.4\left(\mathrm{NHCH}_{2} \mathrm{Ar}\right), 55.3\left(\mathrm{PMB} \mathrm{OCH}_{3}\right), 99.97(\mathrm{C}-6)$, 100.03 (C-5), 108.2 (C-3), 111.2 (C-4a), 112.1 (C-5'), 114.1 (PMB C-3"), 128.9 (PMB C-2"), 130.1 (PMB C-1"), 138.5 (C-5a), 143.1 (C-2), 144.9 (C-7), 145.8 (C-10a), 149.9 (C-9), 157.0 (C6'), 159.0 (PMB C-4"), 159.3 (C-4'), 159.9 (C-4), 171.1 (C-2').

$\mathbb{R}(\mathrm{KBr}): v_{\max } 3854,1585,1312$.

HRMS (ES): Calcd for $\mathrm{C}_{23} \mathrm{H}_{21} \mathrm{~N}_{6} \mathrm{O}_{2}{ }^{32} \mathrm{~S}\left(\mathrm{MH}^{+}\right)$445.1447, found 445.1402. 
9-Amino-4-hydroxy-5-[2-(methylsulfanyl)pyrimidin-4-yl]pyrido [ $\left.3^{\prime}, 2^{\prime}: 4,5\right]$ pyrrolo[1,2-c] pyrimidime 4.8

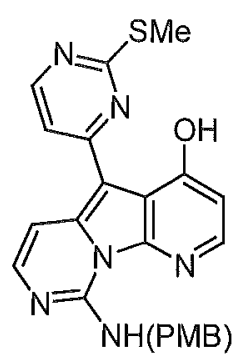<smiles></smiles>

Amine $4.10(9.3 \mathrm{mg}, 0.021 \mathrm{mmol})$ was dissolved in neat $\mathrm{TfOH}(0.1 \mathrm{~mL})$ in a small vial under atmospheric conditions. The vial was capped and the deep red solution was left at it for $19 \mathrm{~h}$. The reaction was cooled to $-10^{\circ} \mathrm{C}$ and carefully quenched with cold, conc aq $\mathrm{NH}_{3}$ solution $(0.1$ $\mathrm{mL}$ ). The mixture was dissolved in $\mathrm{CH}_{2} \mathrm{Cl}_{2} / \mathrm{MeOH}(20: 1)$, washed with $5 \%$ aq $\mathrm{NaHCO}_{3}$ solution and worked up in the usual manner. Flash chromatography on silica, eluting with 1-4.5\% $\mathrm{MeOH} / \mathrm{CH}_{2} \mathrm{Cl}_{2}$, gave the deprotected amine 4.8 as a yellow solid ( $\sim 90 \%$ pure by ${ }^{1} \mathrm{H} \mathrm{NMR}, 6.1$ mg, $80 \%$ ).

Mp: dec at $\sim 265^{\circ} \mathrm{C}$.

$\left.{ }^{1} \mathrm{H} \mathbf{N M R}\left(500 \mathrm{MHz}, 1: 1\left(\mathrm{CD}_{3}\right)_{2} \mathrm{SO} / \mathrm{CDCl}_{3}\right): \delta 2.56(\mathrm{~s}, 3 \mathrm{H}, \mathrm{SCH})_{3}\right), 6.77(\mathrm{~d}, J=5.4 \mathrm{~Hz}, 1 \mathrm{H}, \mathrm{H}-3)$, 7.15 (d, $J=6.3 \mathrm{~Hz}, 1 \mathrm{H}, \mathrm{H}-6), 7.52$ (d, $J=5.9 \mathrm{~Hz}, 1 \mathrm{H}, \mathrm{H}-5$ '), 7.65 (d, $J=6.3 \mathrm{~Hz}, 1 \mathrm{H}, \mathrm{H}-7), 7.97$ (residual $\mathrm{CHCl}_{3}$ ), 8.09 (d, $\left.J=5.4 \mathrm{~Hz}, 1 \mathrm{H}, \mathrm{H}-2\right), 8.33$ (br, $\left.1 \mathrm{H}, \mathrm{NH}\right), 8.44$ (d, $J=5.9 \mathrm{~Hz}, 1 \mathrm{H}, \mathrm{H}-$ 6'), $9.90(\mathrm{br}, 1 \mathrm{H}, \mathrm{NH}), 14.60(\mathrm{~s}, 1 \mathrm{H}, \mathrm{OH})$.

${ }^{13} \mathrm{C} \mathrm{NMR}\left(126 \mathrm{MHz}, 1: 1\left(\mathrm{CD}_{3}\right)_{2} \mathrm{SO} / \mathrm{CDCl}_{3}\right): \delta 13.5\left(\mathrm{SCH}_{3}\right), 78.5\left(\mathrm{t}, \mathrm{CDCl}_{3}\right) 99.0(\mathrm{C}-5), 99.9(\mathrm{C}-$ 6), 107.8 (C-3), 110.9 (C-4a), 112.3 (C-5'), 138.6 (C-5a), 142.9 (C-2), 145.4 (C-7), 145.6 (C10a), 150.5 (C-9), 157.4 (C-6'), 158.8 (C-4'), 159.2 (C-4), 169.9 (C-2').

$\mathbb{R}(\mathrm{RBr}): \mathrm{U}_{\max } 3248,3120-3010$ (series of bands), 1668, 1580, 1568, 1306.

HIRMS (ES): Calcd for $\mathrm{C}_{15} \mathrm{H}_{13} \mathrm{~N}_{6} \mathrm{O}^{32} \mathrm{~S}\left(\mathrm{MH}^{+}\right)$325.0872, found 325.0878 . 
9-Amino-4-methoxy-5-[2-(methylsulfanyl)pyrimidin-4-yl]pyrido[ $\left[3^{\prime}, 2^{\prime}: 4,5\right]$ pyrrolo[1,2-c] pyrimidine 4.12
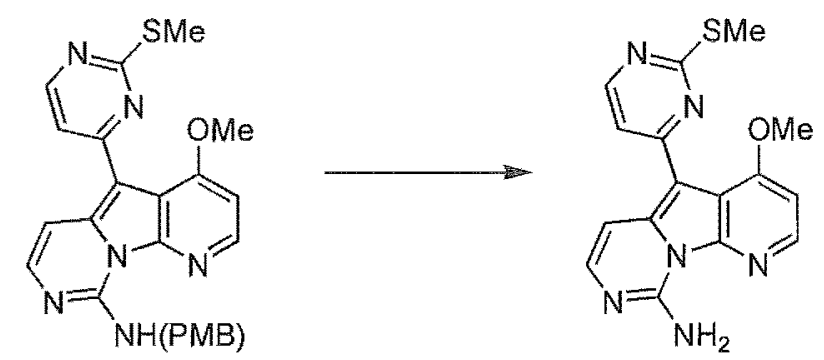

Amine $4.9(7.0 \mathrm{mg}, 0.015 \mathrm{mmol})$ was dissolved in neat $\mathrm{TfOH}(0.05 \mathrm{~mL})$ in a small vial under atmospheric conditions. The vial was capped and the deep red solution was left at $\mathrm{rt}$ for $13 \mathrm{~h}$. The reaction was cooled to $-10^{\circ} \mathrm{C}$ and carefully quenched with cold, conc aq $\mathrm{NH}_{3}$ solution $(0.05$ $\mathrm{mL}$ ). The yellow precipitate was dissolved in $\mathrm{CH}_{2} \mathrm{Cl}_{2}$ and worked up in the usual manner. Flash chromatography on silica, eluting with $5 \% \mathrm{MeOH} / \mathrm{CH}_{2} \mathrm{Cl}_{2}$, gave the deprotected amine 4.12 as a yellow solid ( $5 \mathrm{mg}, 100 \%$ ).

Mp: dec at $179^{\circ} \mathrm{C}$.

${ }^{1} \mathrm{HI} \mathrm{NMR}\left(500 \mathrm{MHz}, \mathrm{CDCl}_{3}\right): \delta 2.65\left(\mathrm{~s}, 3 \mathrm{H}, \mathrm{SCH} H_{3}\right), 4.03\left(\mathrm{~s}, 3 \mathrm{H}, \mathrm{OCH} \mathrm{H}_{3}\right), 5.6-6.6(\mathrm{br}, 1 \mathrm{H}, \mathrm{NH})$, 6.90 (d, $J=5.4 \mathrm{~Hz}, 1 \mathrm{H}, \mathrm{H}-3), 7.39$ (d, $\left.J=5.4 \mathrm{~Hz}, 1 \mathrm{H}, \mathrm{H}-5^{\prime}\right), 7.54$ (d, $J=6.6 \mathrm{~Hz}, 1 \mathrm{H}, \mathrm{H}-7$ ), 7.62 (d, $J=6.6 \mathrm{~Hz}, 1 \mathrm{H}, \mathrm{H}-6), 8.28$ (d, $J=5.4 \mathrm{~Hz}, 1 \mathrm{H}, \mathrm{H}-2), 8.45$ (d, $J=5.4 \mathrm{~Hz}, 1 \mathrm{H}, \mathrm{H}-6$ ), $9.2-10.0$ (br, $1 \mathrm{H}, \mathrm{N} H$ ).

${ }^{13} \mathrm{C} \operatorname{NMR}\left(75 \mathrm{MHz}, \mathrm{CDCl}_{3}\right): \delta 14.1\left(\mathrm{SCH}_{3}\right), 55.6\left(\mathrm{OCH}_{3}\right), 101.1(\mathrm{C}-5), 101.9(\mathrm{C}-3), 102.5(\mathrm{C}-$ 6), $111.6(\mathrm{C}-4 \mathrm{a}), 117.3\left(\mathrm{C}-5^{\prime}\right), 137.3$ (C-5a), 141.98 (C-7 or C-2), 142.04 (C-2 or C-7), 144.6 (C10a), 149.2 (C-9), 155.5 (C-6'), 159.5 (C-4), $161.4\left(\mathrm{C}^{\prime}\right), 171.2\left(\mathrm{C}-2^{\prime}\right)$.

$\mathbb{I R}(\mathrm{KBr}): \mathrm{U}_{\max } 3268,3092-2924$ (series of bands), 1663, 1574, 1011.

MS (EI): $m / z$ (rel intensity) 338 (100), 129 (24), 105 (49), 70 (23), 57 (25).

HRMS (EI): Calcd for $\mathrm{C}_{16} \mathrm{H}_{14} \mathrm{~N}_{6} \mathrm{O}^{32} \mathrm{~S}\left(\mathrm{M}^{+}\right) 338.0950$, found 338.0955 . 


\section{4-Hydroxy-9-(4-methoxybenzylamino)-5-(2-aminopyrimidim-4-yll)pyrido $\left[3^{\prime}, 2^{\prime}: 4,5\right]$ pyrrolo} $[1,2-c]$ pyrimidine 4.13

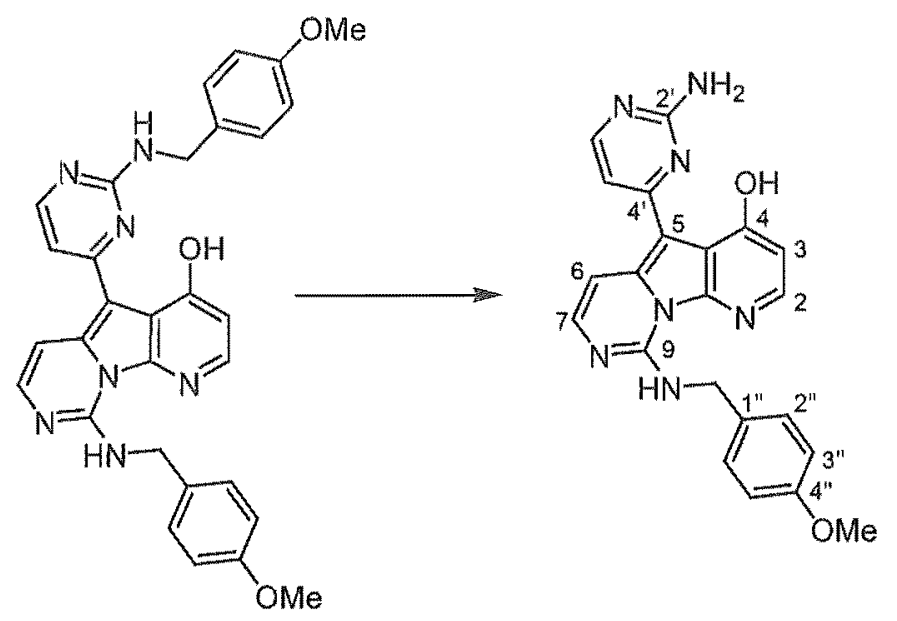

Bis-protected amine $3.35(20 \mathrm{mg}, 0.037 \mathrm{mmol})$ was dissolved in neat TFA $(0.75 \mathrm{~mL})$ under atmospheric conditions, and the stoppered flask was left at it for 5 days. After removal of excess TFA in vacuo, the residue was diluted with $\mathrm{CH}_{2} \mathrm{Cl}_{2}$ and neutralised with sat aq $\mathrm{NaHCO}_{3}$ solution. Repeated extraction with $\mathrm{CH}_{2} \mathrm{Cl}_{2}$ gave a yellow solution which was worked up in the usual manner. Flash chromatography on silica, eluting with 3,4 and then $5 \% \mathrm{MeOH} / \mathrm{CH}_{2} \mathrm{Cl}_{2}$, gave the deprotected amine 4.13 as a yellow solid (10.4 mg, 67\%), and variolin B (1.4 mg, 13\%) respectively.

Mp: dec at $\sim 260^{\circ} \mathrm{C}$.

${ }^{1} \mathrm{H}$ NMR $\left(500 \mathrm{MHz},\left(\mathrm{CD}_{3}\right)_{2} \mathrm{SO}\right): \delta 3.73(\mathrm{~s}, 3 \mathrm{H}, \mathrm{PMB} \mathrm{OCH}), 4.82(\mathrm{~d}, J=5.5 \mathrm{~Hz}, 2 \mathrm{H}$, $\mathrm{NHCH}_{2} \mathrm{Ar}$ ), 6.81 (d, $\left.J=5.7 \mathrm{~Hz}, 1 \mathrm{H}, \mathrm{H}-3\right), 6.92$ (d, $\left.J=8.3 \mathrm{~Hz}, 2 \mathrm{H}, \mathrm{PMB} \mathrm{H}-3 "\right), 7.04$ (s, 2H, $\left.\mathrm{N} H_{2}\right), 7.17$ (d, $\left.J=5.2 \mathrm{~Hz}, 1 \mathrm{H}, \mathrm{H}-5^{\prime}\right), 7.27$ (d, $\left.J=6.7 \mathrm{~Hz}, 1 \mathrm{H}, \mathrm{H}-6\right), 7.38$ (d, $J=8.3 \mathrm{~Hz}, 2 \mathrm{H}, \mathrm{PMB}$ H-2"), 7.70 (d, $J=6.7 \mathrm{~Hz}, 1 \mathrm{H}, \mathrm{H}-7), 8.15(\mathrm{~d}, J=5.7 \mathrm{~Hz}, 1 \mathrm{H}, \mathrm{H}-2), 8.29(\mathrm{~d}, J=5.2 \mathrm{~Hz}, 1 \mathrm{H}, \mathrm{H}-$ 6'), 10.76 (br t, $J=5.5 \mathrm{~Hz}, 1 \mathrm{H}, \mathrm{N} H \mathrm{PMB}), 16.04(\mathrm{~s}, 1 \mathrm{H}, \mathrm{OH}$ ).

${ }^{13} \mathrm{C} \mathrm{NMR}\left(126 \mathrm{MHz},\left(\mathrm{CD}_{3}\right)_{2} \mathrm{SO}\right): 843.6\left(\mathrm{NHCH}_{2} \mathrm{Ar}\right), 55.2(\mathrm{PMB} \mathrm{OCH}), 100.0(\mathrm{C}-5), 100.7(\mathrm{C}-$ 6), 106.2 (C-5'), 107.6 (C-3), 111.1 (C-4a), 114.1 (PMB C-3"), 128.9 (PMB C-2"), 130.6 (PMB C-1"), 136.9 (C-5a), 143.2 (C-2), 144.0 (C-7), 145.0 (C-10a), 149.2 (C-9), 158.4 (C-4'), 158.6 (PMB C-4"), 160.0 (C-4), 160.2 (C-6'), 161.5 (C-2').

$\mathbb{R}(\mathrm{KBr}): v_{\max } 3380,3333,3202,1614-1566$ (series of strong bands), 1481 .

MS (EI): $m / z$ (rel intensity) 413 (72), 293 (28), 121 (100), 91 (27).

HIRMS (EI): Calcd for $\mathrm{C}_{22} \mathrm{H}_{19} \mathrm{~N}_{7} \mathrm{O}_{2}\left(\mathrm{M}^{+}\right) 413.1600$, found 413.1611 . 
9-[2-(Dimethylamino)ethylamino]-4-methoxy-5-[2-(methylsulfanyl)pyrimidin-4-yl]pyrido $\left[3 ;, 2^{\prime}: 4,5\right]$ pyrrolo $[1,2-c]$ pyrimidine 4.21
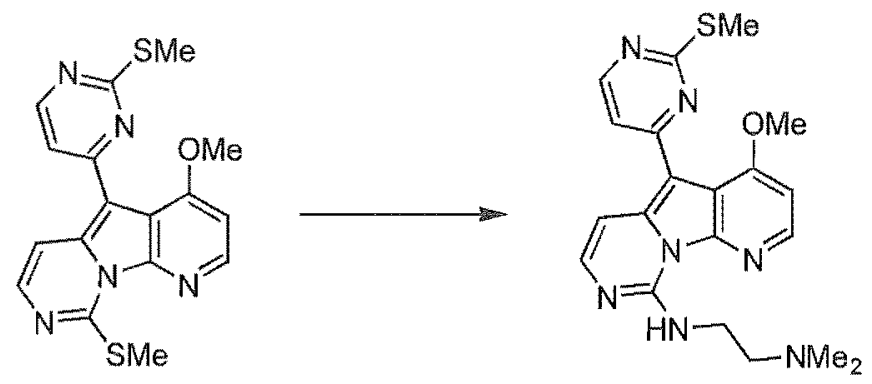

A mixture of core structure $1.63(27.0 \mathrm{mg}, 73.1 \mu \mathrm{mol})$, and $N, N$-dimethylethylenediamine (18 $\mu \mathrm{L}, 0.16 \mathrm{mmol})$ was heated in toluene $(0.6 \mathrm{~mL})$ at $100{ }^{\circ} \mathrm{C}$. Further portions of $N, N$ dimethylethylenediamine $(18 \mu \mathrm{L})$ were added after 48 and $80 \mathrm{~h}$. After 6 days, TLC analysis indicated the complete consumption of starting material. Concentration of the reaction mixture, followed by flash chromatography on silica, eluting with $11-13 \% \mathrm{MeOH} / \mathrm{CH}_{2} \mathrm{Cl}_{2}$, gave amine 4.21 as a yellow-orange solid ( $25.1 \mathrm{mg}, 84 \%$ ).

Mp: $138-141^{\circ} \mathrm{C}$.

${ }^{1} \mathrm{H}$ NMIR $\left(500 \mathrm{MHz}, \mathrm{CDCl}_{3}\right): \delta 2.38\left(\mathrm{~s}, 6 \mathrm{H}, \mathrm{N}\left(\mathrm{CH}_{3}\right)_{2}\right), 2.65(\mathrm{~s}, 3 \mathrm{H}, \mathrm{SCH}), 2.73(\mathrm{t}, J=6.3 \mathrm{~Hz}$, $2 \mathrm{H}, \mathrm{NHCH}_{2} \mathrm{CH}_{2} \mathrm{NMe}_{2}$ ), 3.81 (m, $2 \mathrm{H}, \mathrm{NHCH}_{2} \mathrm{CH}_{2} \mathrm{NMe}_{2}$ ), 4.02 (s, $\left.3 \mathrm{H}, \mathrm{OCH}_{3}\right), 6.87$ (d, $J=5.6$ $\mathrm{Hz}, 1 \mathrm{H}, \mathrm{H}-3), 7.37$ (d, $\left.J=5.4 \mathrm{~Hz}, 1 \mathrm{H}, \mathrm{H}-5^{\prime}\right), 7.55$ (d, $\left.J=6.6 \mathrm{~Hz}, 1 \mathrm{H}, \mathrm{H}-6\right), 7.59$ (d, $J=6.6 \mathrm{~Hz}$, $1 \mathrm{H}, \mathrm{H}-7$ ), 8.25 (d, $J=5.6 \mathrm{~Hz}, 1 \mathrm{H}, \mathrm{H}-2), 8.42$ (d, $\left.J=5.4 \mathrm{~Hz}, 1 \mathrm{H}, \mathrm{H}-6^{\prime}\right), 10.25$ (br t, $J=4.6 \mathrm{~Hz}$, $1 \mathrm{H}, \mathrm{N} H)$.

${ }^{13} \mathrm{C} \mathrm{NMR}\left(126 \mathrm{MHz}, \mathrm{CDCl}_{3}\right): \delta 14.1\left(\mathrm{SCH}_{3}\right), 39.0\left(\mathrm{NHCH}_{2} \mathrm{CH}_{2} \mathrm{NMe}_{2}\right), 45.4\left(\mathrm{~N}\left(\mathrm{CH}_{3}\right)_{2}\right), 55.5$ $\left(\mathrm{OCH}_{3}\right), 58.0\left(\mathrm{NHCH}_{2} \mathrm{CH}_{2} \mathrm{NMe}_{2}\right), 100.7$ (C-5), 101.4 (C-6), 101.7 (C-3), 111.3 (C-4a), 117.2 (C-5'), 137.7 (C-5a), 141.7 (C-2), 142.2 (C-7), 144.9 (C-10a), 148.8 (C-9), 155.4 (C-6'), 159.3 $(\mathrm{C}-4), 161.5\left(\mathrm{C}-4^{\prime}\right), 171.1\left(\mathrm{C}-2^{\prime}\right)$.

IR (KBr): $v_{\max } 3200-2800$ (series of weak bands), 2968, 2945, 2768, 1566, 1312, 1013.

MS (EI): $m / z$ (rel intensity) 409 (5), 351 (25), 338 (100), 58 (47).

HRMS (EI): Calcd for $\mathrm{C}_{20} \mathrm{H}_{23} \mathrm{~N}_{7} \mathrm{O}^{32} \mathrm{~S}\left(\mathrm{M}^{+}\right)$409.1685, found 409.1699 . 
9-[3-(Dimethylamino)propylamino]-4-methoxy-5-[2-(methylsulfanyl)pyrimidirn-4-yll]pyrido $\left[3^{\prime}, 2^{\prime}: 4,5\right]$ pyrrolo $[1,2-c]$ pyrimidine 4.22
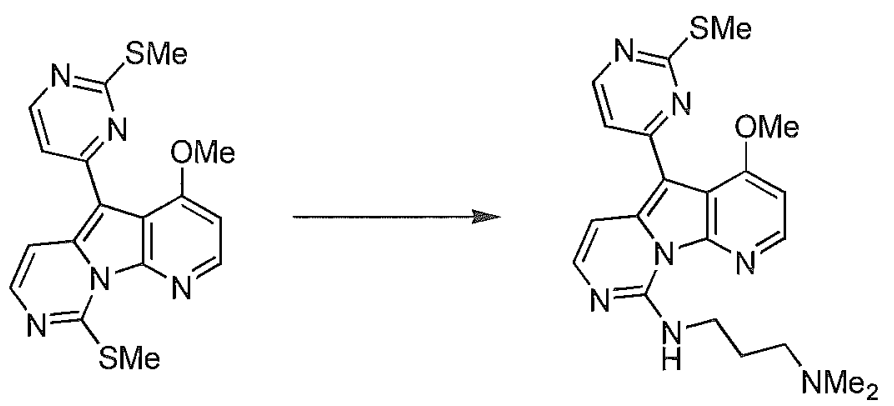

A mixture of core structure $1.63(33.5 \mathrm{mg}, 90.7 \mu \mathrm{mol})$, and 3-(dimethylamino)propylamine (15 $\mu \mathrm{L}, 0.12 \mathrm{mmol})$ was heated in toluene $(0.6 \mathrm{~mL})$ at $100{ }^{\circ} \mathrm{C}$. A further portion of 3(dimethylaminopropylamine) $(11 \mu \mathrm{L})$ was added after $36 \mathrm{~h}$. After $53 \mathrm{~h}$, the reaction mixture was concentrated in vacuo, and the residue was purified by flash chromatography on alumina, eluting with $\mathrm{CH}_{2} \mathrm{Cl}_{2}$ then $2 \% \mathrm{MeOH} / \mathrm{CH}_{2} \mathrm{Cl}_{2}$, to give amine 4.22 as a yellow-orange solid $(34.2 \mathrm{mg}$, $89 \%)$.

Mp: $127-129^{\circ} \mathrm{C}$ (EtOAc).

${ }^{1} \mathbf{H}$ NMR $\left(500 \mathrm{MHz}, \mathrm{CDCl}_{3}\right): \delta 1.96\left(\mathrm{~m}, 2 \mathrm{H}, \mathrm{NHCH}_{2} \mathrm{CH}_{2} \mathrm{CH}_{2} \mathrm{NMe}_{2}\right), 2.28\left(\mathrm{~s}, 6 \mathrm{H}, \mathrm{N}\left(\mathrm{CH}_{3}\right)_{2}\right), 2.47$ (t, $\left.J=7.3 \mathrm{~Hz}, 2 \mathrm{H}, \quad \mathrm{NHCH}_{2} \mathrm{CH}_{2} \mathrm{CH}_{2} \mathrm{NMe}_{2}\right), 2.65(\mathrm{~s}, 3 \mathrm{H}, \quad \mathrm{SCH}), 3.76(\mathrm{~m}, 2 \mathrm{H}$, $\mathrm{NHCH}_{2} \mathrm{CH}_{2} \mathrm{CH}_{2} \mathrm{NMe}_{2}$ ), 4.02 (s, 3H, OCH $\left.{ }_{3}\right), 6.87$ (d, $J=5.9 \mathrm{~Hz}, 1 \mathrm{H}, \mathrm{H}-3$ ), 7.37 (d, $J=5.4 \mathrm{~Hz}$, $\left.1 \mathrm{H}, \mathrm{H}-5^{\prime}\right), 7.54$ (d, $\left.J=6.6 \mathrm{~Hz}, 1 \mathrm{H}, \mathrm{H}-6\right), 7.60$ (d, $\left.J=6.6 \mathrm{~Hz}, 1 \mathrm{H}, \mathrm{H}-7\right), 8.24$ (d, $J=5.9 \mathrm{~Hz}, 1 \mathrm{H}$, H-2), 8.43 (d, $\left.J=5.4 \mathrm{~Hz}, 1 \mathrm{H}, \mathrm{H}-6^{\prime}\right), 10.20$ (br t, $J=5.1 \mathrm{~Hz}, 1 \mathrm{H}, \mathrm{NH}$ ).

${ }^{13} \mathrm{C} \quad \mathbf{N M R}\left(126 \mathrm{MHz}, \mathrm{CDCl}_{3}\right): \quad \delta \quad 14.1 \quad\left(\mathrm{SCH}_{3}\right), \quad 27.2 \quad\left(\mathrm{NHCH}_{2} \mathrm{CH}_{2} \mathrm{CH}_{2} \mathrm{NMe}_{2}\right), \quad 39.3$

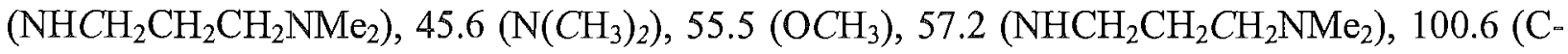
5), 101.1 (C-6), 101.7 (C-3), 111.3 (C-4a), 117.2 (C-5'), 137.8 (C-5a), 141.5 (C-2), 142.4 (C-7), 144.9 (C-10a), 148.8 (C-9), 155.4 (C-6'), 159.3 (C-4), 161.6 (C-4'), 171.1 (C-2').

$\mathbb{I R}(\mathrm{KBr})$ : $\mathrm{v}_{\max } 3240-2760$ (series of weak bands), 2941, 2781, 1570, 1308, 1013.

MS (EI): $m / z$ (rel intensity) 423 (94), 365 (58), 352 (100), 338 (71), 297 (49), 58 (62).

HRMS (EI): Calcd for $\mathrm{C}_{21} \mathrm{H}_{25} \mathrm{~N}_{7} \mathrm{O}^{32} \mathrm{~S}\left(\mathrm{M}^{+}\right)$423.1841, found 423.1842 . 
Procedure for methoxy deprotection of amines $4.21(x=1)$ and $4.22(x=2)$ :
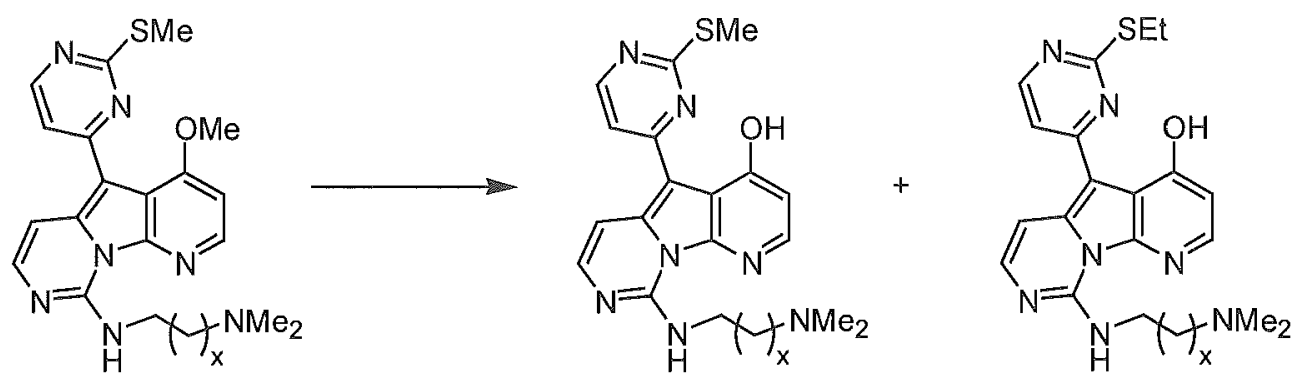

$x=1$ or 2

Sodium ethanethiolate $(80 \%, 52 \mathrm{mg}, 0.49 \mathrm{mmol})$ was dissolved in dry DMF $(0.53 \mathrm{~mL})$. A portion of this $(0.24 \mathrm{~mL}, 0.22 \mathrm{mmol})$ was added to a solution of either $4.21(13.3 \mathrm{mg}, 32.5 \mu \mathrm{mol})$ in DMF (0.37 mL) or $4.22(12.8 \mathrm{mg}, 30.2 \mu \mathrm{mol})$ in DMF $(0.34 \mathrm{~mL})$ and the mixture was stirred at $60{ }^{\circ} \mathrm{C}$ for $20 \mathrm{~h}$. After cooling, aq $\mathrm{NH}_{4} \mathrm{Cl}$ solution was added and the mixture was extracted with $\mathrm{CH}_{2} \mathrm{Cl}_{2}$ and worked up in the usual manner.

The crude product of the first reaction $(\mathrm{x}=1)$ was purified by flash chromatography on silica, eluting with $12-20 \% \mathrm{MeOH} / \mathrm{CH}_{2} \mathrm{Cl}_{2}$ then $20 \% \mathrm{MeOH} / \mathrm{CH}_{2} \mathrm{Cl}_{2}+0.5 \% \mathrm{NEt}_{3}$, to give:

\section{9-[2-(Dimethylamino)ethylamino]-4-hydroxy-5-[2-(methylsulfanyl)pyrimidim-4-yl]pyrido} $\left[3^{\prime}, 2^{\prime}: 4,5\right]$ pyrrolo[1,2-c]pyrimidine 4.23 , contaminated with $\sim 6 \%$ of the SEt-substituted analogue $4.25(11.7 \mathrm{mg}, 91 \%)$.

Mp: $175-178^{\circ} \mathrm{C}$, sweats at $171^{\circ} \mathrm{C}\left(\mathrm{MeOH} / \mathrm{CH}_{2} \mathrm{Cl}_{2}\right)$.

$\left.{ }^{1} \mathrm{HI} \mathrm{NMR}\left(500 \mathrm{MHz}, \mathrm{CDCl}_{3}\right): \delta 2.39\left(\mathrm{~s}, 6 \mathrm{H}, \mathrm{N}\left(\mathrm{CH}_{3}\right)_{2}\right), 2.62(\mathrm{~s}, 3 \mathrm{H}, \mathrm{SCH})_{3}\right), 2.73(\mathrm{t}, J=6.3 \mathrm{~Hz}$, $2 \mathrm{H}, \mathrm{NHCH}_{2} \mathrm{CH}_{2} \mathrm{NMe}_{2}$ ), 3.78 (m, 2H, $\left.\mathrm{NHCH}_{2} \mathrm{CH}_{2} \mathrm{NMe}_{2}\right), 6.80$ (d, $\left.J=5.6 \mathrm{~Hz}, 1 \mathrm{H}, \mathrm{H}-3\right), 6.89$ (d, $J=6.3 \mathrm{~Hz}, 1 \mathrm{H}, \mathrm{H}-6), 7.27$ (d, $\left.J=5.8 \mathrm{~Hz}, 1 \mathrm{H}, \mathrm{H}-5^{\prime}\right), 7.66$ (d, $\left.J=6.3 \mathrm{~Hz}, 1 \mathrm{H}, \mathrm{H}-7\right), 8.07$ (d, $J=$ $5.6 \mathrm{~Hz}, 1 \mathrm{H}, \mathrm{H}-2$ ), 8.36 (d, $J=5.8 \mathrm{~Hz}, 1 \mathrm{H}, \mathrm{H}-6$ '), 10.74 (br t, $J=5.0 \mathrm{~Hz}, 1 \mathrm{H}, \mathrm{NH}), 14.65$ (s, $1 \mathrm{H}$, $\mathrm{OH})$.

${ }^{13} \mathrm{C}$ NMR $\left.\left(126 \mathrm{MHz}, \mathrm{CDCl}_{3}\right): \delta 13.9\left(\mathrm{SCH}_{3}\right), 39.2\left(\mathrm{NHCH}_{2} \mathrm{CH}_{2} \mathrm{NMe}_{2}\right), 45.5\left(\mathrm{~N}_{\left(\mathrm{CH}_{3}\right)}\right)_{2}\right), 58.0$ $\left(\mathrm{NHCH}_{2} \mathrm{CH}_{2} \mathrm{NMe}_{2}\right.$ ), 99.6 (C-6), 99.8 (C-5), 108.1 (C-3), 111.0 (C-4a), 112.0 (C-5'), 138.4 (C5a), 143.0 (C-2), 144.9 (C-7), 145.7 (C-10a), 149.8 (C-9), 156.9 (C-6'), 159.1 (C-4'), 159.7 (C4), $170.9\left(\mathrm{C}-2^{\prime}\right)$. 
$\mathbb{R}(\mathrm{KBr}): \mathrm{v}_{\max } 3182,2977-2733$ (series of weak bands), 1823, 1610, 1590, 1312, 1013.

MS (EI): $m / z$ (rel intensity) 395 (11), 338 (43), 337 (54), 324 (47), 58 (100).

HRMS (EI): Calcd for $\mathrm{C}_{19} \mathrm{H}_{21} \mathrm{~N}_{7} \mathrm{O}^{32} \mathrm{~S}\left(\mathrm{M}^{+}\right)$395.1528, found 395.1534 .

The crude product of the second reaction $(\mathrm{x}=2)$ was purified by flash chromatography on silica, eluting with $20 \% \mathrm{MeOH} / \mathrm{CH}_{2} \mathrm{Cl}_{2}$ then $30 \% \mathrm{MeOH} / \mathrm{CH}_{2} \mathrm{Cl}_{2}+0.5 \% \mathrm{NEt}_{3}$, to give:

\section{9-[3-(Dimethylamino)propylamino]-4-hydroxy-5-[2-(methylsulfanyl)pyrimidin-4-yl]pyrido} $\left[3^{\prime}, 2^{\prime}: 4,5\right]$ pyrrolo[1,2-c]pyrimidine 4.24 , contaminated with $\sim 8 \%$ of the SEt-substituted analogue $4.26(7.2 \mathrm{mg}, 58 \%)$.

Mp: $66-69^{\circ} \mathrm{C}$.

${ }^{1} \mathrm{H}$ NMR $\left(500 \mathrm{MHz}, \mathrm{CDCl}_{3}\right): \delta 1.96\left(\mathrm{~m}, 2 \mathrm{H}, \mathrm{NHCH}_{2} \mathrm{CH}_{2} \mathrm{CH}_{2} \mathrm{NMe}_{2}\right), 2.30\left(\mathrm{~s}, 6 \mathrm{H}, \mathrm{N}\left(\mathrm{CH}_{3}\right)_{2}\right), 2.49$ $\left(\mathrm{t}, J=7.2 \mathrm{~Hz}, 2 \mathrm{H}, \quad \mathrm{NHCH}_{2} \mathrm{CH}_{2} \mathrm{CH}_{2} \mathrm{NMe}_{2}\right), 2.62(\mathrm{~s}, 3 \mathrm{H}, \quad \mathrm{SCH}), 3.73(\mathrm{~m}, 2 \mathrm{H}$, $\left.\mathrm{NHCH}_{2} \mathrm{CH}_{2} \mathrm{CH}_{2} \mathrm{NMe}_{2}\right), 6.79(\mathrm{~d}, J=5.8 \mathrm{~Hz}, 1 \mathrm{H}, \mathrm{H}-3), 6.88(\mathrm{~d}, J=6.7 \mathrm{~Hz}, 1 \mathrm{H}, \mathrm{H}-6), 7.28$ (d, $J=$ $\left.5.8 \mathrm{~Hz}, 1 \mathrm{H}, \mathrm{H}-5^{\prime}\right), 7.66$ (d, $\left.J=6.7 \mathrm{~Hz}, 1 \mathrm{H}, \mathrm{H}-7\right), 8.05$ (d, $\left.J=5.8 \mathrm{~Hz}, 1 \mathrm{H}, \mathrm{H}-2\right), 8.36$ (d, $J=5.8$ $\mathrm{Hz}, 1 \mathrm{H}, \mathrm{H}-6$ ) $) 10.68$ (br t, $J=5.2 \mathrm{~Hz}, 1 \mathrm{H}, \mathrm{NH}$ ), 14.67 (s, 1H, OH).

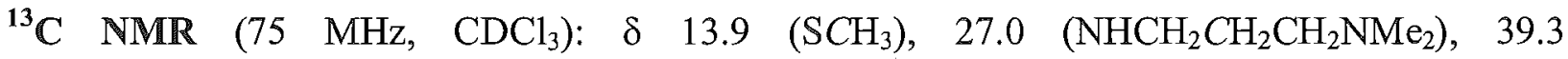
$\left.\left(\mathrm{NHCH}_{2} \mathrm{CH}_{2} \mathrm{CH}_{2} \mathrm{NMe}_{2}\right), 45.4\left(\mathrm{~N}_{(} \mathrm{CH}_{3}\right)_{2}\right), 57.1\left(\mathrm{NHCH}_{2} \mathrm{CH}_{2} \mathrm{CH}_{2} \mathrm{NMe}_{2}\right), 99.5(\mathrm{C}-6), 99.7(\mathrm{C}-5)$, 108.1 (C-3), 111.1 (C-4a), 112.0 (C-5'), 138.5 (C-5a), 142.8 (C-2), 145.0 (C-7), 145.7 (C-10a), 149.8 (C-9), 156.9 (C-6'), 159.1 (C-4'), 159.7 (C-4), 170.8 (C-2').

IR (KBr): $\mathbf{v}_{\max } 2968-2767$ (series of weak bands), 1570, 1310.

HRMS (ES): Calcd for $\mathrm{C}_{20} \mathrm{H}_{24} \mathrm{~N}_{7} \mathrm{O}^{32} \mathrm{~S}\left(\mathrm{MH}^{+}\right) 410.1763$, found 410.1759 . 


\section{9-[3-(Dimethylamino)propylamino]-4-methoxy-5-[2-(methylsulfinyl)pyrimidin-4-yl]pyrido} $\left[3^{\prime}, 2{ }^{\prime}: 4,5\right]$ pyrrolo[1,2-c]pyrimidine 4.28
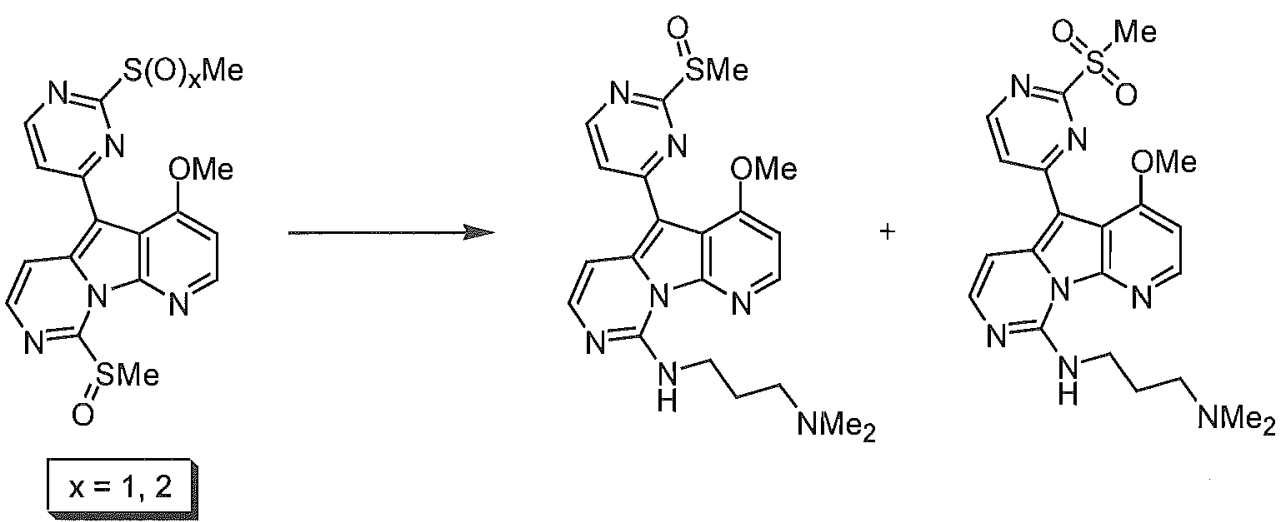

A crude mixture of $\mathbf{3 . 3 2}$ was prepared from $1.63(55.2 \mathrm{mg}, 0.149 \mathrm{mmol})$ and $m$-CPBA $(\sim 70 \%$, $64.5 \mathrm{mg}, 0.26 \mathrm{mmol}$ ), as previously described (except that the reaction was carried out at -60 to 0 $\left.{ }^{\circ} \mathrm{C}\right)$. The product was dissolved in $\mathrm{CDCl}_{3}(1.0 \mathrm{~mL})$, and a portion $(0.70 \mathrm{~mL}, 0.10 \mathrm{mmol})$ was transferred to an NMR tube containing $N, N$-dimethylaminopropylamine $(13 \mu \mathrm{L}, 0.10 \mathrm{mmol})$. A ${ }^{1} \mathrm{H}$ NMR spectrum obtained $10 \mathrm{~min}$ after mixing showed essentially complete mono-substitution. After $17 \mathrm{~h}$ at rt, the reaction mixture was concentrated in vacuo and partially purified by flash chromatography on silica, eluting with $20 \% \mathrm{MeOH} / \mathrm{CHCl}_{3}$ then $20-30 \% \mathrm{MeOH} / \mathrm{CHCl} 3+1 \%$ $\mathrm{NEt}_{3}$. Further purification was achieved on reversed phase silica, eluting with $50 \% \mathrm{MeOH} /$ water $+0.1 \%$ TFA. After evaporation of the solvents, the protonated product was dissolved in $5 \%$ $\mathrm{MeOH} / \mathrm{CH}_{2} \mathrm{Cl}_{2}$ and neutralised with anhydrous $\mathrm{K}_{2} \mathrm{CO}_{3}$. Filtration and evaporation of the filtrate gave a yellow solid (19.6 mg) which was shown by ${ }^{1} \mathrm{H}$ NMR spectroscopy to be a $\sim 8: 1$ mixture of sulfoxide 4.28 and the corresponding sulfone 4.29 . Spectroscopic data for the sulfoxide is given below:

$\left.{ }^{1} \mathrm{H} \mathbb{N M R}\left(500 \mathrm{MHz}, \mathrm{CDCl}_{3}\right): \delta 1.97\left(\mathrm{~m}, 2 \mathrm{H}, \mathrm{NHCH}_{2} \mathrm{CH}_{2} \mathrm{CH}_{2} \mathrm{NMe}_{2}\right), 2.29\left(\mathrm{~s}, 6 \mathrm{H}, \mathrm{N}\left(\mathrm{CH}_{3}\right)\right)_{2}\right), 2.49$ $\left(\mathrm{t}, J=7.1 \mathrm{~Hz}, 2 \mathrm{H}, \mathrm{NHCH}_{2} \mathrm{CH}_{2} \mathrm{CH}_{2} \mathrm{NMe}_{2}\right), 3.01$ (s, $\left.3 \mathrm{H}, \mathrm{S}(\mathrm{O}) \mathrm{CH}_{3}\right), 3.77$ (m, 2H, $\mathrm{NHCH}_{2} \mathrm{CH}_{2} \mathrm{CH}_{2} \mathrm{NMe}_{2}$ ), 4.07 (s, 3H, $\left.\mathrm{OCH}_{3}\right), 6.92$ (d, $\left.J=5.6 \mathrm{~Hz}, 1 \mathrm{H}, \mathrm{H}-3\right), 7.71$ (d, $J=6.6 \mathrm{~Hz}$, $1 \mathrm{H}, \mathrm{H}-7), 7.80$ (d, $J=6.6 \mathrm{~Hz}, 1 \mathrm{H}, \mathrm{H}-6), 7.83$ (d, $\left.J=5.6 \mathrm{~Hz}, 1 \mathrm{H}, \mathrm{H}-5{ }^{\prime}\right), 8.27$ (d, $J=5.6 \mathrm{~Hz}, 1 \mathrm{H}$, $\mathrm{H}-2), 8.69$ (d, $J=5.6 \mathrm{~Hz}, 1 \mathrm{H}, \mathrm{H}-6$ ), 10.29 (br t, $J=5.1 \mathrm{~Hz}, 1 \mathrm{H}, \mathrm{N} H$ ).

${ }^{13} \mathrm{C}$ NMR (75 MHz, CDCl 3 ): $\delta 26.9\left(\mathrm{NHCH}_{2} \mathrm{CH}_{2} \mathrm{CH}_{2} \mathrm{NMe}_{2}\right), 39.2\left(\mathrm{NHCH}_{2} \mathrm{CH}_{2} \mathrm{CH}_{2} \mathrm{NMe}_{2}\right), 40.1$ $\left(\mathrm{S}(\mathrm{O}) \mathrm{CH}_{3}\right), 45.3\left(\mathrm{~N}_{\left.\left(\mathrm{CH}_{3}\right)_{2}\right),} 55.6\left(\mathrm{OCH}_{3}\right), 57.0\left(\mathrm{NHCH}_{2} \mathrm{CH}_{2} \mathrm{CH}_{2} \mathrm{NMe}_{2}\right), 99.4(\mathrm{C}-5), 101.3(\mathrm{C}-6)\right.$, 
101.9 (C-3), 110.7 (C-4a), $121.1(\mathrm{C}-5), 139.2$ (C-5a), $141.6(\mathrm{C}-2), 144.0$ (C-7), 144.9 (C-10a), $148.6(\mathrm{C}-9), 156.0\left(\mathrm{C}-6^{\prime}\right), 158.9(\mathrm{C}-4), 162.6\left(\mathrm{C}-4^{\prime}\right), 172.0\left(\mathrm{C}-2^{\prime}\right)$.

HRMS (ES): Calcd for $\mathrm{C}_{21} \mathrm{H}_{26} \mathrm{~N}_{7} \mathrm{O}_{2}{ }^{32} \mathrm{~S}\left(\mathrm{MH}^{+}\right) 440.1869$, found 440.1873 . 


References


$1 \quad$ Hung, D. T.; Jamison, T. F.; Schreiber, S. L. Chem. Biol. 1996, 3, 623.

Sneader, W. Drug Prototypes and their Exploitation, John Wiley \& Sons, Chichester, 1996, pp 85-96.

3

See reference 2, pp 469489.

Urban, S.; Hickford, J. H.; Blunt, J. W.; Munro, M. H. G. Current Org. Chem. 2000, 4, 765.

Rayl, A. J. S. The Scientist 1999, 13, 1.

Williams, D. H.; Stone, M. J.; Hauck, P. R.; Rahman, S. K. J. Nat. Prod. 1989, 52, 1189.

Faulkner, D. J. Tetrahedron 1977, 33, 1421.

Henkel, T.; Brunne, R. M.; Muller, H.; Reichel, F.; Angew. Chem. Int. Ed. Eng. 1999, 38, 643.

Lin, Y.-Y.; Risk, M.; Ray, S. M.; Van Engen, D.; Clardy, J.; Golik, J.; James, J. C.; Nakanishi, K. J. Am. Chem. Soc. 1981, 103, 6773.

(a) Tetrodotoxin: The $3^{\text {rd }}$ IUPAC Symposium on the Chemistry of Natural Products, 13 April, 1964 (Kyoto, Japan). Tsuda, K.; Ikuma, S.; Kawamura, M.; Tachikawa, R.; Sakai, K.; Tamura, C.; Amakasu, O. Chem. Pharm. Bull. (Tokyo) 1964, 12, 1357. Tsuda, K.; Naturwissenschaften 1966, 53, 171. Goto, T.; Kishi, Y.; Takahashi, S.; Hirata, Y. Tetrahedron 1965, 21, 2059. Woodward, R. B. Pure Appl. Chem. 1964, 9, 49. Mosher, H. S.; Fuhrman, F. A.; Buchwald, H. D.; Fischer, H. D. Science 1964, 144, 1100.

(b) Chlorophyll c: Dougherty, R. C.; Strain, H. H.; Svec, W. A.; Uphaus, R. A.; Katz, J. J. J. Am. Chem. Soc. 1966, 88, 5037; Dougherty, R. C.; Strain, H. H.; Svec, W. A.; Uphaus, R. A.; Katz, J. J. J. Am. Chem. Soc. 1970, 92, 2826

(c) 1-Methylisoguanosine: Quinn, R. J.; Gregson, R. P.; Cook, A. F,; Bartlett, R. T. Tetrahedron Lett. 1980, 21, 567.

(d) Sceptrin: Walker, R. P.; Faulkner, D. J. J. Am. Chem. Soc. 1981, 103, 6772.

(e) Purealin: Nakamura, H.; Wu, H.; Kobayashi, J.; Nakamura, Y.; Ohizumi, Y.; Hirata, Y. Tetrahedron Lett. 1985, 26, 4517.

(f) Manzamine A: Sakai, R.; Higa, T; Jefford, C. W.; Bernardinelli, G. J. Am. Chem. Soc. $1986,108,6404$. 
(g) Cephalostatin 1: Pettit, G. R.; Inoue, M.; Kamano, X.; Herald, D. L.; Arm, C.; Dufresne, C.; Christie, N. D.; Schmidt, J. M.; Doubek, D. L.; Krupa, T. S. J. Am. Chem. Soc. $1988,110,2006$.

(h) Eleutherobin: Lindel, T.; Jensen, P. R.; Fenical, W.; Long, B. H.; Casazza, A. M.; Carboni, J.; Fairchild, C. R. J. Am. Chem. Soc. 1997, 119, 8744.

11 Sneader, W. Drug Prototypes and their Exploitation, John Wiley \& Sons, Chichester, $1996, \mathrm{p} 459$ and references therein.

Patil, A. D.; Freyer, A. J.; Taylor, P. B.; Carté, B.; Zuber, G.; Johnson, R. K.; Faulkner, D. J. J. Org. Chem. 1997, 62, 1814.

(a) Snider, B. B.; Busuyek, M. V. J. Nat. Prod. 1999, 62, 1707.

(b) Black, G. P.; Murphy, P. J.; Thornhill, A. J.; Walshe, N. D. A.; Zanetti, C. Tetrahedron $1999,55,6547$.

Cohen, F; Overman, L. E. J. Am. Chem. Soc. 2001, 123, 10782.

(a) Li, J.; Jeong, S.; Esser, L.; Harran, P. G. Angew. Chem. Int. Ed. 2001, 40, 4765.

(b) Li, J.; Burgett, A. W. G.; Esser, L.; Amezcua, C.; Harran, P. G. Angew. Chem. Int. Ed. $2001,40,4770$.

Lindquist, N.; Fenical, W.; Van Duyne, G. D.; Clardy, J.; J. Am. Chem. Soc. 1991, 113, 2303.

For a leading reference, see ref $15 \mathrm{a}$.

Hirata, Y.; Uemura, D. Pure Appl. Chem. 1986, 58, 701.

Aicher, T. D.; Buszek, K. R.; Fang, F. G.; Forsyth, C. J.; Jung, S. H.; Kishi, Y.; Matelich, M. C.; Scola, P. M.; Spero, D. M.; Yoon, S. K. J. Am. Chem. Soc. 1992, 114, 3162.

Stamos, D. P.; Chen, S. S.; Kishi, Y. J. Org. Chem. 1997, 62, 7552.

Wright, A. E.; Forleo, D. A.; Gunawardana, G. P.; Gunasekera, S. P.; Koehn, F. E.; McConnell, O. J. J. Org. Chem. 1990, 55, 4508.

Rinehart, K. L.; Holt, T. G.; Fregeau, N. L.; Stroh, J. G.; Keifer, P. A.; Sun, F.; Li, L. H.; Martin, D. G. J. Org. Chem. 1990, 55, 4512. 
Borman, S. Chem. Eng. News 2001, Sept. 24, 41.

Takebayashi, Y.; Pourquier, P.; Zimonjic, D. B.; Nakayama, K.; Emmert, S.; Ueda, T.; Urasaki, Y.; Kanzaki, A. Akiyama, S.; Popescu, N.; Kraemer, K. H.; Pommier, Y. Nat. Med. 2001, 7, 961.

Zewail-Foote, M.; Li, V.-S.; Kohn, H.; Bearss, D.; Guzman, M.; Hurley, L. H. Chem. Biol. 2001, 8, 1033.

Jaspars, M. Chem. Ind. 1999, 51.

(a) Corey, E. J.; Gin, D. Y. Tetrahedron Lett. 1996, 37, 7163.

(b) Corey, E. J.; Gin, D. Y.; Kania, R. S. J. Am. Chem. Soc. 1996, 118, 9202.

(c) Saito, N.; Tashiro, K.; Maru, Y.; Yamaguchi, K.; Kubo, A. J. Chem. Soc., Perkin Trans. 1 1997, 53.

(d) Endo, A.; Kann, T.; Fukuyama, T. Synlett 1999, 1103.

Martinez, E. J.; Owa, T.; Schreiber, S. L.; Corey, E. J. Proc. Natl. Acad. Sci. USA 1999, 96,3496 .

Martinez, E. J;; Corey, E. J. Org Lett. 2000, 2, 993.

Cuevas, C.; Pérez, M.; Martín, M. J.; Chicharro, J. L.; Fernández-Rivas, C.; Flores, M.; Francesch, A.; Gallego, P.; Zarzuelo, M.; de la Calle, F.; García, J.; Polanco, C.; Rodríguez, I.; Manzanares, I. Org Lett. 2000, 2, 2545.

(a) Perry, N. B.; Ettouati, L.; Litaudon, M.; Blunt, J. W.; Munro, M. H. G. Tetrahedron $1994,50,3987$.

(b) Trimurtulu, G.; Faulkner, D. J.; Perry, N. B.; Ettouati, L.; Litaudon, M.; Blunt, J. W.; Munro, M. H. G.; Jameson, G. B. Tetrahedron 1994, 50, 3993.

Capuano, L.; Schrepfer, H. J.; Müller, K.; Roos, H. Chem. Ber. 1974, 107, 929.

Rothwell, D. R. The Madrid Protocol and its Relationship with the Antarctic Treaty System, Antarctic and Southern Oceans Law and Policy Occasional Paper 5, Blay, S. K. N.; Piotrowicz, R. W.; Tsamenyi, B. W.; Davis, B. D. Eds., Faculty of Law, University of Tasmania, Hobart, 1992. 
(a) Minguez, J. M.; Vaquero, J. J.; Alvarez Builla, J.; Castaño, O.; André, J. L. J. Org. Chem. 1999, 64, 7788.

(b) Álvarez, M.; Fernández, D; Joule, J. A. Synthesis 1999, 615.

(c) Álvarez, M.; Fernández, D.; Joule, J. A. J. Chem. Soc., Perkin Trans. 1 1999, 249.

(d) Fresneda, P. M.; Molina, P.; Bleda, J. A. Tetrahedron 2001, 57, 2355.

(e) Álvarez, M.; Fernández, D.; Joule, J. A. J. Chem. Soc., Perkin Trans. $12002,471$.

(a) Fresneda, P. M.; Molina, P.; Delgado, S.; Bleda, J. A. Tetrahedron Lett. 2000, 41, 4777 .

(b) Molina, P.; Fresneda, P. M.; Delgado, S.; Bleda, J. A. Tetrahedron Lett. 2002, 43, 1005.

Mendiola, J.; Minguez, J. M; Alvarez-Builla, J.; Vaquero, J. J. Org. Lett. 2000, 2, 3253.

Álvarez, M.; Fernández, D.; Joule, J. A. Tetrahedron Lett. 2001, 42, 315.

(a) Anderson, R. J.; Morris, J. C. Tetrahedron Lett. 2001, 42, 311.

(b) Anderson, R. J.; Morris, J. C. Tetrahedron Lett. 2001, 42, 8697.

Desarbre, E.; Coudret, S.; Meheust, C.; Mérour, J-Y. Tetrahedron 1997, 53, 3637.

Comins, D. L.; LaMunyon, D. H. Tetrahedron Lett. 1988, 29, 773.

Hemetsberger, H.; Knittel, D.; Weidmann, H. Monatsh. Chem. 1970, 101, 161.

For a review on the use of iminophosphoranes in aza-Wittig reactions for the synthesis of fused heterocycles, see Molina, P.; Vilaplana, M. J. Synthesis 1994, 1197.

Isolation of the Meridianins: Franco, L. H.; de K. Joffé, E. B.; Puricelli, L.; Tatian, M.; Seldes, A. M.; Palermo, J. A. J. Nat. Prod. 1998, 61, 1130.

Katritzky, A. R.; Akutagawa, K. J. Am. Chem. Soc. 1986, 108, 6808.

Corey, E. J.; Cheng, K.-M. The Logic of Chemical Synthesis, John Wiley and Sons, New York, 1989 , pp 4446.

For a recent example, see: Adlington, R. M.; Baldwin, J. E.; Catterick, D.; Pritchard, G. E. J. Chem. Soc, Perkin Trans, 1 1999, 855.

Greene, T. W.; Wuts, P. G. Protective Groups in Organic Synthesis, Wiley-Interscience, New York, 1991, $2^{\text {nd }}$ edition, pp 146-148. 
(a) Freedman, H. H. in: Carbonium Ions, vol. $I V$, Olah, G. A.; Schleyer, P. v. R., Eds., Wiley-Interscience, Toronto, pp 1501-1578.

(b) Wada, M.; Konishi, H.; Kirishima, K.; Takeuchi, H.; Natsume, S.; Erabi, T. Bull. Chem. Soc. Jpn. 1997, 70, 2737.

(c) Wada, M.; Watanabe, T.; Natsume, S.; Mishima, H.; Kirishima, K.; Erabi, T. Bull. Chem. Soc. Jpn. 1995, 68, 3233.

(d) Wada, M.; Mishima, H.; Watanabe, T.; Natsume, S.; Konishi, H.; Kirishima, K.; Hayase, S.; Erabi, T. Bull. Chem. Soc. Jpn. 1995, 68, 243.

(e) Smith, R. J.; Miller, T. M.; Pagni, R. M. J. Org. Chem. 1982, 47, 4181.

(f) Martin. J. C.; Smith, R. G. J. Am. Chem. Soc. 1964, 86, 2252.

Gibson, H. W.; Lee, S. H.; Engen, P. T.; Lecavalier, P.; Sze, J.; Shen, Y. X.; Bheda, M. J. Org. Chem. 1993, 58, 3748.

For recent reviews on the directed metallation of azines and diazines, see: Mongin, F,; Quéguiner, G. Tetrahedron 2001, 57, 4059. Turck, A.; Plé, N.; Mongin, F.; Quéguiner, G. Tetrahedron 2001, 57, 4489.

For pyridines, see: Gilman, H.; Melstrom, D. S. J. Am. Chem. Soc. 1946, 68, 103. Gilman, H.; Spatz, S. M. J. Org. Chem. 1951, 16, 1485. Mallet, M.; Quéguiner, G. Tetrahedron 1986, 42, 2253. Peterson, M. A.; Mitchell, J. R. J. Org. Chem. 1997, 62, 8237.

For pyrimidines, see: Langley, B. W. J. Am. Chem. Soc. 1956, 78, 2136. Hirschberg, A.; Peterkofsky, A.; Spoerri, P. E. J. Heterocycl. Chem. 1965, 2, 209. Hetrz, H. S.; Kabacinski, F. F.; Spoerri, P. E. J. Heterocycl. Chem. 1969, 6, 239. Párkányi, C.; Cho, N. S.; Yoo, G. S. J. Organomet. Chem. 1988, 342, 1. Shimura, A.; Momotake, A.; Togo, H.;Yokoyama, M. Synthesis, 1999, 495. Leprêtre, A.; Turck, A.; Plé, N.; Quéguiner, G. Tetrahedron $2000,56,3709$. Gronowitz, S.; Röe, J. Acta Chem. Scand. 1965, 19, 1741.

Leprềtre, A.; Turck, A.; Plé, N.; Knochel, P.; Quéguiner, G. Tetrahedron 2000, 56, 265. 
Plé, N.; Turck, A.; Quéguiner, G.; Glassl, B. Liebigs Ann. Chem. 1993, 583.

Plé, N.; Turck, A.; Couture, K.; Quéguiner, G. J. Org. Chem. 1995, 60, 3781.

See, for example: Marzi, E.; Bigi, A.; Schlosser, M. Eur. J. Org. Chem. 2001, 1371. Choppin, S.; Gros, P.; Fort, Y. Eur. J. Org. Chem. 2001, 603.

Majeed, A. J.; Antonsen, Ø.; Benneche, T.; Undheim, K. Tetrahedron 1989, 45, 993.

Hoffmann, C.; Faure, A. Bull. Soc. Chim. Fr. 1966, 2316.

NIST/EPA/NIH MS Database, Version 4.0, May 1992.

Jung, M. E.; Blum, R. B. Tetrahedron Lett. 1977, 3791.

Wakefield, B. J. Organomagnesium Methods in Organic Synthesis, Academic Press, London, 1995, p 152. Wardell, J. L. Comprehensive Organomet. Chem. 1982, 1, 50.

Harden, D. B.; Mokrosz, M. J.; Strekowski, L. J. Org. Chem. 1988, 53, 4137.

Suzuki, M.; Noyori, R. in: Organocopper Reagents: A Practical Approach, Taylor, R. J. K., Ed., Oxford University Press, New York, 1994, pp 201-206.

Kursanov, D. N.; Parnes, Z. N.; Loim, N. M. Synthesis, 1974, 633.

Gordon, P. E.; Fry, A. J. Tetrahedron Lett. 2001, 42, 831.

Hartwig, W. Tetrahedron 1983, 39, 2609.

Barton, D. H. R.; McCombie, S. W. J. Chem. Soc., Perkin Trans. 1 1975, 1574.

Conway, R. J.; Nagel, J. P.; Stick, R. V.; Tilbrook, D. M. G. Aust. J. Chem. 1985, 38, 939.

Dolan, S. C.; MacMillan, J. J. Chem. Soc., Chem. Commun. 1985, 1588.

Sundaralingam, M.; Chwang, A. K. in: Carbonium Ions, vol. V, Olah, G. A.; Schleyer, P. v. R., Eds., Wiley-Interscience, Toronto, pp 2435-2443.

Lyle, R. E.; Anderson, P. S. Adv. Heterocycl. Chem. 1966, 6, 45. Lyle, R. E.; Boyce, C. B. J. Org. Chem. 1974, 39, 3708.

Lau, C. K.; Dufresne, C.; Bélanger, P. C.; Piétré, S.; Scheigetz, J. J. Org. Chem. 1986, 51,3038 . 
76 Olah, G. A.; Prakash, G. K. S. Synthesis $1978,397$.

77 Lee, W. Y.; Park, C. H.; Kim, Y. D. J. Org. Chem. 1992, 57, 4074.

78 Gribble, G. W.; Leese, R. M. Synthesis 1977, 172.

79 Peterson, P. E.; Casey, C. J. Org. Chem. 1964, 29, 2325.

Brewster, J. H.; Osman, S. F.; Bayer, H. O.; Hopps, H. B. J. Org. Chem. 1964, 29, 121.

81 Kemp, W. Organic Spectroscopy, Macmillan, London, 1991, $3^{\text {rd }}$ edition, p 125.

82 See reference $81, \mathrm{p} 62$.

Brown, D. J,; Ford, P. W. J. Chem. Soc. (C) 1967, 568.

Brown, D. J.; Foster, R. V. Aust. J. Chem. 1966, 19, 1487.

Kos, N. J.; van der Plas, H. C. J. Org. Chem. 1980, 45, 2942.

Kos, N. J.; van der Plas, H. C, van Veldhuizen, B. J. Org. Chem. 1979, 44, 3141.

Kondo, T.; Okamoto, K.; Yamamoto, M.; Goto, T. Tetrahedron 1986, 42, 199.

Shishoo, C. J.; Jain, K. S. J. Heterocycl. Chem. 1992, 29, 883.

89

Masquelin, T.; Sprenger, D.; Baer, R.; Gerber, F.; Mercadal, Y. Helv. Chim. Acta 1998, 81,646 .

(a) Hydrogen peroxide: Tarbell, D. S.; Weaver, C. J. Am. Chem. Soc. 1941, 63, 2939.

(b) m-CPBA: See references 47,83

(c) Oxone ${ }^{\circledR}$ : Trost, B. M.; Curran, D. P. Tetrahedron Lett. 1981, 22, 1287. Hirano, M.; Tomaru, J; Morimoto, T. Bull. Chem. Soc. Jpn. 1991, 64, 3752. Greenhalgh, R. P. Synlett 1992, 235.

(d) Sodium metaperiodate: Traynellis, V. J.; Yoshikawa, Y.; Tarka, S. M.; Livingston (Jr.), J. R. J. Org. Chem. 1973, 38, 3986. Gupta, D. N.; Hodge, P.; Davies, J. E. J. Chem. Soc., Perkin Trans. 1 1981, 2970.

(e) Sulfuryl chloride/wet silica: Hojo, M.; Masuda, R. Tetrahedron Lett. 1976, 613.

(f) Potassium permanganate: Menger, F. M.; Lee, C. J. Org. Chem. 1979, 44, 3446. Gokel, G. W.; Gerdes, H. M.; Dishong, D. M. J. Org. Chem. 1980, 45, 3634. 
(g) Tetrabutylammonium periodate: Santaniello, E.; Manzocchi, A.; Farachi, C. Synthesis 1980, 563. Takata, T.; Ando, W. Tetrahedron Lett. 1983, 24, 3631.

(h) Titanium trichloride/hydrogen peroxide: Watanabe, Y.; Numata, T.; Oae, S. Synthesis $1981,204$.

(i) Sodium perborate: McKillop, A.; Tarbin, J. A. Tetrahedron Lett. 1983, 24, 1505.

(j) Tetrapropylammonium perruthenate: Guertin, K. R.; Kende, A. S. Tetrahedron Lett. $1993,34,5369$.

Hurst, D. T.; Johnson, M. Heterocycles 1985, 23, 611.

Still, W. C.; Khan, M.; Mitra, A. J. Org. Chem. 1978, 43, 2923.

Kocieński, P. J.; Protecting Groups, Thieme, New York, 2000, Corr. edition, pp 220-225 and references therein.

Krische, M. J.; Lehn, J.-M.; Kyritsakas, N.; Fischer, J.; Wegelius, E. K.; Nissinen, M. J.; Rissanen, K. Helv. Chim. Acta 1998, 81, 1921.

Smith, A. B.; Rano, T. A.; Chida, N.; Sulikowski, G. A.; Wood, J. L. J. Am. Chem. Soc. $1992,114,8008$.

Kasum, B.; Prager, R. H. Aust. J. Chem. 1983, 36, 1455.

Normant, H. Angew. Chem., Int. Ed. Engl. 1967, 6, 1046 and references therein. Murayama, E.; Kikuchi, T.; Sasaki, K. Chem. Lett. 1984, 1897. Oehlschlager, A. C.; Czyzewska, E.; Aksela, R.; Pierce, H. D. Jr. Can. J. Chem. 1986, 64, 1407.

Mukhopadhyay, T.; Seebach, D. Helv. Chim. Acta 1982, 65, 385. See also: Lipshutz, B. H.; Moretti, R.; Crow, R. Tetrahedron Lett. 1989, 30, 15. Seebach, D.; Maestro, M. A.; Sefkow, M.; Adam, G.; Hintermann, S.; Neidlein, A. Liebigs Ann. Chem. 1994, 701.

Bartlett, P. D.; Goebel, C. V.; Weber, W. P. J. Am. Chem. Soc. 1969, 91, 7425.

Gilkerson, W. R.; Jackson, M. D. J. Am. Chem. Soc. 1979, 101, 4096. Cohen, T.; Abraham, W. D.; Myers, M. J. Am. Chem. Soc. 1987, 109, 7293. Reich, H. J.; Borst, J. P.; Dykstra, R. R.; Green, D. P. J. Am. Chem. Soc. 1993, 115, 8728.

Corey, E. J.; Beames, D. J. J. Am. Chem. Soc. 1959, 81, 2674. Neumann, H.; Seebach, D. Tetrahedron Lett. 1976, 4839. 
(a) Imamoto, T.; Sugiura, Y.; Takiyama, N. Tetrahedron Lett. 1984, 25, 4233.

(b) Denmark, S. E.; Edwards, J. P.; Nicaise, O. J. Org. Chem. 1993, 58, 569.

Talik, Z. Roczniki Chem. 1962, 36, 1313. Chem. Abstr. 1963, 59, 6358b.

Walters, M. A.; Shay, J. Tetrahedron Lett. 1995, 36, 7575.

Connon, S. J.; Hegarty, A. F. Tetrahedron Lett. 2001, 42, 735.

Finger, G. C.; Starr, L. D. J. Am. Chem. Soc. 1959, 81, 2674.

Lundy, S. D. Honours Thesis, University of Canterbury, 2001.

Ochiai, E. J. Org. Chem. 1953, 18, 534.

Okuda, S.; Robison, M. M. J. Am. Chem. Soc. 1959, 81, 740.

Sieburth, S. McN.; Lin, C.-H.; Rucando, D. J. Org. Chem. 1999, 64, 950.

Andersen, K.; Begtrup, M. Acta Chem. Scand. 1992, 46, 1130.

Brunet, J.-J.; Sidot, C.; Caubere, P. Tetrahedron Lett. 1981, 22, 1013. Brunet, J.-J.; Sidot, C.; Caubere, P. J. Org. Chem. 1983, 48, 1166. Foà, M.; Francalanci, F.; Bencini, E.; Gardano, A. J. Organomet. Chem. 1985, 285, 293.

Hirota, Y.; Ryang, M.; Tsutsumi, S. Tetrahedron Lett. 1971, 1531.

Radinov, R.; Haimova, M.; Simova, E. Synthesis 1986, 886. Lucas, P.; Mehdi, N. E.; Ho, H. A.; Bélanger, D.; Breau, L. Synthesis 2000, 1253.

McOmie, J. F. W.; Watts, M. L.; West, D. E. Tetrahedron 1968, 24, 2289.

Feutrill, G. I.; Mirrington, R. N. Aust. J. Chem. 1972, 25, 1719. Hannan, R. L.; Barber, R. B.; Rapoport, H. J. Org. Chem. 1979, 44, 2153. Kende, A. S.; Rizzi, J. P. Tetrahedron Lett. 1981, 22, 1779.

Goodwin, S.; Smith, A. F.; Horning, E. C.; J. Am. Chem. Soc. 1959, 81, 1903.

Dalton, L. K.; Demerac, S.; Elmes, B. C.; Loder, J. W.; Swan, J. M.; Teitei, T. Aust. J. Chem. 1967, 20, 2715.

See Zhang, Q.; Shi, C.; Zhang, H.-R.; Wang, K. K. J. Org. Chem. 2000, 65, 7977. 
Froelich-Ammon, S. J.; Patchan, M. W.; Osheroff, N.; Thompson, R. B. J. Biol. Chem. $1995,270,14998$.

121

Voet, D.; Voet, J. G.; Pratt, C. W. Fundamentals of Biochemistry, John Wiley \& Sons, New York, 1999, p 749.

122

(a) Todd, A. K.; Adams, A.; Thorpe, J. H.; Denny, W. A.; Wakelin, L. P. G.; Cardin, C. J. J. Med. Chem. 1999, 42, 536.

(b) Gamage, S. A.; Spicer, J. A.; Atwell, G. J.; Finlay, G. J.; Baguley, B. C.; Denny, W. A. J. Med. Chem. 1999, 42, 2383.

(c) Adams, A.; Guss, J. M.; Collyer, C. A.; Denny, W. A.; Wakelin, L. P. G. Biochemistry 1999, 38, 9221 .

Fortune, J. M.; Velea, L.; Graves, D. E.; Utsugi, T.; Yamada, Y.; Osheroff, N. Biochemistry, 1999, 38, 15580.

Armarego, W. L. F.; Perrin, D. D. Purification of Laboratory Chemicals, ButterworthHeinemann, Oxford, $1997,4^{\text {th }}$ edition.

Burfield, D. R.; Smithers, R. H. J. Org. Chem. 1978, 43, 3966.

Watson, S. C.; Eastham, J. F. J. Organomet. Chem. 1967, 9, 165.

A Guide to IUPAC Nomenclature of Organic Compounds: Recommendations 1993, Richer, J.-C. Senior Ed., Blackwell Scientific Publications, London, 1993, p 68. Aldrich Handbook of Fine Chemicals and Laboratory Equipment, 2000-2001, p 1227. 